\title{
Modelos parcialmente lineares \\ com erros simétricos autoregressivos de primeira ordem
}

\author{
Carlos Eduardo Martins Relvas \\ DisSERTAÇÃO APRESENTADA \\ $\mathrm{AO}$ \\ Instituto DE MATEMÁticA E EstatísticA \\ DA \\ UnIVERSIDADE DE SÃO PAUlO \\ PARA \\ OBTENÇÃO DO TÍTULO \\ $\mathrm{DE}$ \\ Mestre EM Cî̂NCIAS \\ Programa: Mestrado em Estatística \\ Orientador: Prof. Dr. Gilberto Alvarenga Paula
}

Durante o desenvolvimento deste trabalho o autor recebeu auxílio financeiro da $\mathrm{CNPq}$

São Paulo, fevereiro de 2013 


\title{
Modelos parcialmente lineares com erros simétricos autoregressivos de primeira ordem
}

\author{
Esta dissertação contém as correções e alterações \\ sugeridas pela Comissão Julgadora durante a defesa \\ realizada por Carlos Eduardo Martins Relvas em 19/04/2013. \\ O original encontra-se disponível no Instituto de \\ Matemática e Estatística da Universidade de São Paulo.
}

Comissão Julgadora:

- Prof. Dr. Gilberto Alvarenga Paula - IME-USP

- Prof. Dr. Gérman Mauricio Ibacache Pulgar - UV-Chile

- Prof. Dr. Francisco José de Azevêdo Cysneiros - UFPE 


\section{Agradecimentos}

Agradeço, primeiramente, a Deus, que sempre me acompanhou e me ajudou a conquistar todos os objetivos que eu busquei, desde a entrada na universidade à obtenção do bacharelado e agora no decorrer do mestrado.

Ao Professor Dr. Gilberto Alvarenga Paula por toda sua dedicação, seus ensinamentos e orientação que foram fundamentais para a conclusão deste trabalho e na minha formação como Estatístico.

Aos meus pais Manuel e Marlene Relvas e minha irmã Ana Paula por todo o apoio, carinho e companheirismo fornecido durante toda a minha vida.

À minha namorada Renata por toda a compreensão e carinho nos momentos mais difíceis para a conclusão deste trabalho.

A todos os professores do IME-USP, em especial, os do departamento de estatística que fizeram parte de toda a minha formação acadêmica.

A todos os meus amigos de graduação e pós graduação que sempre me ensinaram muito.

A toda minha família e amigos que sempre me animaram nos momentos mais complicados. 


\section{Resumo}

\section{Modelos parcialmente lineares com erros simétricos autoregressi- vos de primeira ordem}

Neste trabalho, apresentamos os modelos simétricos parcialmente lineares $\mathrm{AR}(1)$, que generalizam os modelos parcialmente lineares para a presença de erros autocorrelacionados seguindo uma estrutura de autocorrelação AR(1) e erros seguindo uma distribuição simétrica ao invés da distribuição normal. Dentre as distribuições simétricas, podemos considerar distribuições com caudas mais pesadas do que a normal, controlando a curtose e ponderando as observações aberrantes no processo de estimação. A estimação dos parâmetros do modelo é realizada por meio do critério de verossimilhança penalizada, que utiliza as funções escore e a matriz de informação de Fisher, sendo todas essas quantidades derivadas neste trabalho. O número efetivo de graus de liberdade e resultados assintóticos também são apresentados, assim como procedimentos de diagnóstico, destacando-se a obtenção da curvatura normal de influência local sob diferentes esquemas de perturbação e análise de resíduos. Uma aplicação com dados reais é apresentada como ilustração. 
Palavras-chave: modelos não paramétricos, modelos semiparamétricos, erros autoregressivos, modelos simétricos, splines naturais cúbicos, estimação robusta, modelos t de Student, algoritmo back-fitting, análise de resíduos, pontos de alavanca, influência local. 


\section{Abstract \\ Symmetric partially linear models with first-order autoregressive errors}

In this master dissertation, we present the symmetric partially linear models with $A R(1)$ errors that generalize the normal partially linear models to contain autocorrelated errors $\mathrm{AR}(1)$ following a symmetric distribution instead of the normal distribution. Among the symmetric distributions, we can consider heavier tails than the normal ones, controlling the kurtosis and downweighting outlying observations in the estimation process. The parameter estimation is made through the penalized likelihood by using score functions and the expected Fisher information. We derive these functions in this work. The effective degrees of freedom and asymptotic results are also presented as well as the residual analysis, highlighting the normal curvature of local influence under different perturbation schemes. An application with real data is given for illustration.

Palavras-chave: non-parametric models, semi-parametric models, autoregressive errors, symmetric models, natural cubic splines, robust estimation, Student- 
t models, back-fitting algorithm, residual analysis, leverage, local influence. 


\section{Sumário}

Lista de Figuras $\quad$ xi

Lista de Tabelas $\quad$ xvii

1 Introdução 1

1.1 Preliminares ....................... . . 1

1.2 Proposta de trabalho . . . . . . . . . . . . . 3

1.3 Modelos parcialmente lineares com erros normais $\mathrm{AR}(1) \ldots 4$

1.4 Exemplo ilustrativo . . . . . . . . . . . . . . . . . . . . 4

2 Revisão de modelos semiparamétricos autogressivos 9

2.1 Modelos semiparamétricos . . . . . . . . . . . . . . . . . 9

2.1.1 Modelos não paramétricos . . . . . . . . . . . . 10

2.1.2 Modelos semiparamétricos . . . . . . . . . . . 17

2.2 Modelos parcialmente lineares com erros simétricos . . . . . . . 19

2.2.1 Distribuições simétricas univariadas . . . . . . . . . . 19

2.2.2 Modelos de regressão linear com erros simétricos . . . . . 21

2.2.3 Modelo parcialmente linear com erros simétricos . . . . . 21 
2.2.4 Funções escore e informação de Fisher ...... . 23

2.2.5 Procedimentos de estimação . . . . . . . . . . . . . 25

2.3 Modelos com erros autoregressivos . . . . . . . . . . . . . . 26

2.4 Conclusões do capítulo . . . . . . . . . . . . . . . . . . . . . . . . . 29

3 Modelos parcialmente lineares simétricos $\mathrm{AR}(1) \quad 31$

3.1 Descrição do modelo . . . . . . . . . . . . . . . . . . . . 31

3.1.1 Derivação dos dois primeiros momentos . . . . . . . . . 32

3.2 Funções escore . . . . . . . . . . . . . . . . . . . . . . 34

3.3 Informação de Fisher . . . . . . . . . . . . . . . . . . . 36

3.3.1 Derivação das Hessianas . . . . . . . . . . . . . . 37

3.3.2 Derivação das informações de Fisher . . . . . . . . . . . 39

3.4 Procedimentos de Estimação . . . . . . . . . . . . . . . . . . . . 42

3.4.1 Escolha do parâmetro de suavização . . . . . . . . . . . 45

3.5 Resultados assintóticos . . . . . . . . . . . . . . . 47

3.6 Conclusões do Capítulo . . . . . . . . . . . . . . . . . . . . . . . 48

4 Análise de Diagnóstico 49

4.1 Análise de diagnóstico do modelo linear normal . . . . . . . . 50

4.2 Resíduos . . . . . . . . . . . . . . . . . 55

4.3 Pontos de alavanca . . . . . . . . . . . . . . . 56

4.4 Influência local . . . . . . . . . . . . . . . . . . . 58

4.4.1 Perturbação aditiva na resposta do modelo . . . . . . . . 59

4.4.2 Perturbação aditiva em uma variável contínua do modelo 60 
4.4 .3 Ponderação de casos . . . . . . . . . . . . . . . . . . 61

4.5 Conclusões do capítulo . . . . . . . . . . . . . . 62

5 Aplicações $\quad 63$

5.1 Séries de temperaturas . . . . . . . . . . . . 63

5.2 Modelo linear com erros autoregressivos . . . . . . . . . . 65

5.3 Modelo parcialmente linear normal $\mathrm{AR}(1) \ldots . . . . . .70$

5.4 Modelos parcialmente lineares t de Student $\mathrm{AR}(1) \ldots$. . . . . 79

5.5 Influência local . . . . . . . . . . . . . . . . 89

5.5.1 Análise confirmatória . . . . . . . . . . . . . 95

5.6 Previsão . . . . . . . . . . . . . . . . . . . . 97

5.7 Conclusões do capítulo . . . . . . . . . . . . . . . . . . . 98

6 Conclusões finais 101

6.1 Considerações finais . . . . . . . . . . . . . . . . . . 101

6.2 Trabalhos futuros . . . . . . . . . . . . . . 102

A Derivação dos momentos impostos pelo modelo 103

A.1 Derivação dos dois primeiros momentos . . . . . . . . . . . 103

A.2 Derivação de correlação . . . . . . . . . . . . . . . . . 104

$\begin{array}{ll}\text { B Funções escore } & 107\end{array}$

$\begin{array}{ll}\text { C Matriz de informação de Fisher } & 109\end{array}$

C.1 Matriz Hessiana . . . . . . . . . . . . . . . . . . . . . 109

C.2 Informação de Fisher . . . . . . . . . . . . . . . . . . 111 
x SUMÁRIO

D Diagnóstico

D.1 Matriz de alavancas . . . . . . . . . . . . . . . 117

D.2 Influência local . . . . . . . . . . . . . . . . . . . . 120

D.2.1 Perturbação aditiva na resposta do modelo . . . . . . . . 120

D.2.2 Perturbação aditiva em uma variável contínua do modelo 123

D.2.3 Ponderação de casos . . . . . . . . . . . . . . . . 125

$\begin{array}{llr}\text { E Aplicações } & 129\end{array}$

$\begin{array}{ll}\text { Referências Bibliográficas } & 131\end{array}$ 


\section{Lista de Figuras}

1.1 Gráfico normal de probabilidades para os resíduos padronizados referente ao modelo (1.1) ajustado aos dados sobre temperatura de Ubatuba e Cananéia. . . . . . . . . . . . . . . . . 6

1.2 Funções de autocorrelação e autocorrelação parcial para os resíduos do modelo (1.1) ajustado aos dados sobre temperaturas de Ubatuba e Cananéia. . . . . . . . . . . . . . . . . . 7

2.1 Splines estimados, para a temperatura de Ubatuba ao longo do tempo, considerando polinômios lineares, quadráticos e cúbicos . 12

2.2 Diferentes valores do parâmetro de suavização - Temperatura de Ubatuba. . . . . . . . . . . . . . . . . . . 15

5.1 Gráfico de dispersão entre as temperaturas médias mensais de Ubatuba e Cananéia. . . . . . . . . . . . . . . . . . 64

5.2 Temperatura média mensal de Ubatuba ao longo do tempo. . . 65 
5.3 Histograma dos resíduos padronizados referente ao ajuste do modelo linear normal com erros autoregressivos aos dados de temperaturas. ..................... 67

5.4 Resíduo padronizado ao longo do tempo referente ao ajuste do modelo linear com erros autoregressivos aos dados de temperaturas, com um spline estimado aos dados em vermelho. . . . . . 68

5.5 Funções de autocorrelações para os resíduos padronizados referente ao ajuste do modelo linear com erros autoregressivos aos dados de temperaturas. . . . . . . . . . . . . . . 69

5.6 O primeiro gráfico mostra o valor de AIC para diferentes valores de $\alpha$, enquanto que o segundo mostra o número de graus de liberdade para cada um desses ajustes, considerando o modelo parcialmente linear normal com erros autoregressivos $\mathrm{AR}(1)$. . . 71

5.7 O primeiro gráfico mostra o valor do AIC corrigod para diferentes valores de $\alpha$, enquanto que o segundo mostra o critério de validação cruzada generalizada para cada um desses ajustes, considerando o modelo parcialmente linear normal com erros autoregressivos $\mathrm{AR}(1) \ldots \ldots \ldots \ldots 72$

5.8 Histograma dos resíduos padronizados para os dados de temperatura considerando o modelo parcialmente linear normal com erros autoregressivos AR(1). . . . . . . . . . . 74 
5.9 Resíduos padronizados ao longo do tempo para os dados de temperatura considerando o modelo parcialmente linear normal com erros autoregressivos $\mathrm{AR}(1)$, com um spline estimado aos dados em vermelho. . . . . . . . . . . . . . 75

5.10 Estimativas do efeito do tempo para os dados de temperatura considerando o modelo parcialmente linear normal com erros autoregressivos $\mathrm{AR}(1) \ldots \ldots \ldots \ldots \ldots$

5.11 Gráfico normal de probabilidades para os resíduos padronizados referente ao modelo parcialmente linear normal com erros autoregressivos $\mathrm{AR}(1)$ ajustado aos dados de temperaturas. . . . 77

5.12 Funções de autocorrelação e autocorrelação parcial para os resíduos padronizados referente ao modelo parcialmente linear normal com erros autoregressivos AR(1) ajustado aos dados de tem-

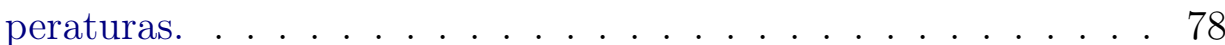

5.13 Os gráficos mostram o valor de AIC para diferentes valores de $\alpha$ e de $v$ (graus de liberdade da t de Student) considerando o modelo parcialmente linear t de Student com erros autoregressivos $\mathrm{AR}(1) \ldots \ldots \ldots \ldots \ldots \ldots \ldots$

5.14 O primeiro gráfico mostra o valor de AIC para diferentes valores de $\alpha$, enquanto que o segundo mostra o número de graus de liberdade efetivo para cada um desses ajustes, considerando o modelo parcialmente linear t de Student com 3 graus de liberdade com erros autoregressivos AR(1). . . . . . . . . . . 81 
5.15 O primeiro gráfico mostra o valor do AIC corrigod para diferentes valores de $\alpha$, enquanto que o segundo mostra o critério de validação cruzada generalizada para cada um desses ajustes, considerando o modelo parcialmente linear t de Student com 3 graus de liberdade com erros autoregressivos AR(1) . . . . . . . 82

5.16 Histograma dos resíduos padronizados para os dados de temperatura considerando o modelo parcialmente linear t de Student com erros autoregressivos $\mathrm{AR}(1) . \ldots \ldots \ldots$. . . . . . . 83

5.17 Resíduos padronizados ao longo do tempo para os dados de temperatura considerando o modelo parcialmente linear t de Student com erros autoregressivos $\mathrm{AR}(1)$, com um spline estimado aos dados em vermelho. . . . . . . . . . . . . . 84

5.18 Estimativas do efeito do tempo para os dados de temperatura considerando o modelo parcialmente linear t de Student com erros autoregressivos $\mathrm{AR}(1) \ldots \ldots \ldots \ldots$

5.19 Gráfico normal de probabilidades para os resíduos padronizados referente ao modelo parcialmente linear t de Student com erros autoregressivos $\mathrm{AR}(1)$ ajustado aos dados de temperaturas. . . . 86

5.20 Funções de autocorrelação e autocorrelação parcial para os resíduos padronizados referente ao modelo parcialmente linear $t$ de Student com erros autoregressivos AR(1) ajustado aos dados de temperaturas. . . . . . . . . . . . . 87 
5.21 Temperatura de Ubatuba observada e estimada (em vermelho pontilhado) referente ao modelo parcialmente linear t de Stu-

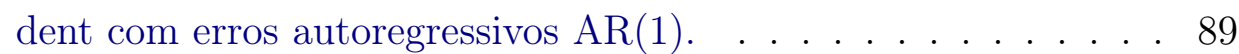

5.22 Gráficos de índices $C_{i}$ para $\boldsymbol{\theta}$ sob o esquema de ponderação de casos para o ajustes dos modelos parcialmente lineares com erros autoregressivos AR(1) t de Student e normal. . . . . . . . . . 90

5.23 Gráficos de índices $C_{i}$ para $\boldsymbol{\beta}$ sob o esquema de ponderação de casos para o ajustes dos modelos parcialmente lineares com erros autoregressivos AR(1) t de Student e normal. . . . . . . . 90

5.24 Gráficos de índices $C_{i}$ para $\mathbf{f}$ sob o esquema de ponderação de casos para o ajustes dos modelos parcialmente lineares com erros autoregressivos AR(1) t de Student e normal. . . . . . . . . . 91

5.25 Gráficos de índices $C_{i}$ para $\phi$ sob o esquema de ponderação de casos para o ajustes dos modelos parcialmente lineares com erros autoregressivos AR(1) t de Student e normal. . . . . . . . . . . 91

5.26 Gráficos de índices $C_{i}$ para $\rho$ sob o esquema de ponderação de casos para o ajustes dos modelos parcialmente lineares com erros autoregressivos AR(1) t de Student e normal. . . . . . . . . . 92

5.27 Gráficos dos resíduos padronizados pela ordem das observações para o ajustes dos modelos parcialmente lineares com erros autoregressivos AR(1) normal e t de Student. . . . . . . . . 93

5.28 Gráfico de dispersão entre as temperaturas de Ubatuba e Cananéia destacando os pontos aberrantes. . . . . . . . . . . . . 94 
5.29 Gráfico de dispersão entre as temperaturas médias mensais de Ubatuba e Cananéia apenas para os 7 primeiros anos de dados. 96

5.30 Série observada de temperatura de Ubatuba (em preto); série estimada pelo modelo (em vermelho); valores previstos para a série de temperaturas (em azul pontilhado); valores observados não utilizados no processo de estimação (em preto pontilhado); a linha verde pontilhada indica o ponto que foi utilizado no ajuste do modelo. . . . . . . . . . . . . . . . . . . . . 99 


\section{Lista de Tabelas}

1.1 Estimativas dos parâmetros modelo (1.1) ajustado aos dados sobre temperaturas de Ubatuba e Cananéia. . . . . . . . . . 5

2.1 Expressões para $W_{g}(u)$ e $W_{g}^{\prime}(u)$ para algumas distribuições simétricas. . . . . . . . . . . . . . . . . 24

2.2 Valores de $d_{g}, f_{g}$ e $\xi$ para algumas distribuições simétricas. . . . 25

5.1 Intervalos de confiança de $95 \%$ para as estimativas da parte paramétrica dos três ajustes apresentados referentes aos dados de temperatura. LI - Limite inferior, LS - Limite superior . . . . 88

5.2 Intervalos de confiança de $95 \%$ para as estimativas da parte paramétrica considerando todos os dados e um subconjunto menor, supondo erros normais. LI - Limite inferior, LS - Limite superior 95

5.3 Intervalos de confiança de 95\% para as estimativas da parte paramétrica considerando todos os dados e um subconjunto menor, supondo erros t de Student. LI - Limite inferior, LS - Limite

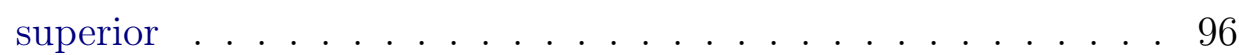


xviii LISTA DE TABELAS

E.1 Estimativas dos parâmetros do modelo linear com erros autoregressivos AR(1) ajustado aos dados sobre temperaturas. . . . . . 129

E.2 Estimativas dos parâmetros do modelo parcialmente linear normal com erros autoregressivos AR(1) ajustado aos dados de temperaturas. . . . . . . . . . . . . . . . . . . 129

E.3 Estimativas dos parâmetros do modelo parcialmente linear t de Student (3 graus de liberdade) com erros autoregressivos AR(1) ajustado aos dados de temperaturas. . . . . . . . . . . 130 


\section{Capítulo 1}

\section{Introdução}

\section{$1.1 \quad$ Preliminares}

Os modelos lineares com erros normais têm sido amplamente aplicados no contexto de regressão para o estudo de relações entre variáveis, em que a variável resposta é supostamente normal nos níveis das variáveis explicativas. Uma excelente referência no assunto, onde podem ser encontradas as principais metodologias de regressão com erros normais, é o livro de Montgomery et al. [2007]. Todavia, a suposição de normalidade (condicional) é muito díficil de ser verificada descritivamente, sendo necessário, na maioria dos casos, recorrer a análises de resíduos. Assim, afastamentos da normalidade podem ocorrer e quando ignorados podem levar a resultados inferenciais incorretos. Dentre os vários tipos de afastamento da normalidade, um dos mais comuns é aquele em que há indícios de que a distribuição dos erros tem caudas mais pesadas do que a distribuição normal, sugerindo o uso de distribuições (condicionais) com tais características, tais como a distribuição t de Student (vide Lange et al. [1989]) em que a curtose é controlada pelos graus de liberdade. Uma ampla classe de distribuições simétricas contínuas com caudas mais leves e mais pesadas do que as caudas da distribuição normal, tais como a t de Student, exponencial potência, logísticas I e II e normal contaminada, dentre outras, é discutida em Fang et al. [1990].

Nos últimos 10 anos vários trabalhos envolvendo modelos lineares com erros simétricos foram publicados. Por exemplo, Galea et al. [2003], Galea et al. [2005], Cysneiros e Paula [2005] e Cysneiros et al. [2007] discutem, respectivamente, modelos lineares, modelos não lineares, modelos lineares com parâmetros restritos e modelos heteroscedásticos com erros simétricos. Já em 
Cysneiros et al. [2005] há uma revisão de modelos simétricos com aplicações desenvolvidas no software computacional R e em Paula e Cysneiros [2009] são apresentadas aplicações na área de Finanças.

As suposições de variância constante e ausência de correlação entre os erros podem também não ser verificadas em muitas situações práticas de aplicação de modelos de regressão com erros normais, havendo algumas propostas de avaliar e modelar a heteroscedasticidade nesses modelos (vide, por exemplo, Cook e Weisberg [1983]; Verbyla [1993]; Lin e Wei [2003]). Extensões para modelos de regressão com erros simétricos são mais recentes, como por exemplo em Taylor e Verbyla [2004] em que os parâmetros de posição e dispersão são modelados conjuntamente em modelos de regressão com erros t de Student e em Cysneiros et al. [2005] em que esses mesmos parâmetros são modelados conjuntamente em modelos simétricos. Por outro lado, a avaliação da estrutura de correlação em modelos de regressão com erros normais, como por exemplo, estrutura autoregressiva, tem sido estudada sob erros normais (vide Montgomery et al. [2007], Cap. 13; Tsai [1986]; Lin e Wei [2004]). Recentemente, Cao et al. [2010] estudaram conjuntamente heteroscedasticidade e autocorrelação em modelos de regressão com erros simétricos.

A introdução de componentes não paramétricos como, por exemplo, Bsplines, em modelos de regressão tem sido proposta em algumas situações no sentido de flexibilizar a relação entre determinadas variáveis explicativas contínuas e a variável resposta, permitindo a inclusão de relações não lineares em modelos lineares. Em algumas situações, pode não haver interesse em estabelecer uma relação paramétrica entre a variável resposta e a variável contínua, mas sim em controlar o efeito da última através de alguma função não paramétrica. Os modelos parcialmente lineares contemplam tais situações em que o componente sistemático é formado por um termo paramétrico e outro termo não paramétrico. Por exemplo, em Kim et al. [2002] há uma discussão sobre modelos parcialmente lineares com erros normais e Ibacache-Pulgar e Paula [2011] estendem os resultados de Kim et al. [2002] para modelos parcialmente lineares com erros t de Student. Um livro clássico no assunto é Green e Silverman [1994]. Rigby e Stasinopoulos [2005] apresentam uma ampla classe de modelos com respostas univariadas em que o componente sistemático pode ser formado por termos paramétricos, não paramétricos e aleatórios. Essa classe, denominada GAMLSS (Generalized Aditive Models for Location, Scale and Shape), contempla, em particular, modelos parcialmente lineares com erros simétricos. Códigos em $\mathrm{R}$ estão disponíveis para o ajuste e análise desses modelos.

Há, também, trabalhos discutindo modelos parcialmente lineares com estru- 
tura de autocorrelação nos erros. Chen e You [2005] apresentam procedimentos de estimação de mínimos quadrados generalizados para controlar a autocorrelação. Mais recentemente, Dabo-Niang e Guillas [2010] discutem a consistência das estimativas de mínimos quadrados generalizados supondo uma estrutura de autocorrelação autoregressiva de ordem $d$ e sugerem o procedimento de Prais - Winston para estimar os parâmetros do modelo.

Como a maioria dos modelos são construídos baseados em algumas suposições, uma das mais importantes etapas de modelagem é realizar uma análise de diagnóstico completa para verificar possíveis fugas dessas suposições. Existem na literatura diversas técnicas de diagnóstico, uma das mais elaboradas e utilizadas nos últimos tempos é o método de influência local, proposto por Cook [1986] que busca verificar se pequenas perturbações nos dados ou no modelo causam mudanças significativas nas inferências realizadas. Nos últimos 20 anos, mais de 400 artigos foram publicados sobre o tema. Paula [2010] apresenta os principais artigos publicados.

Em relação aos temas estudados nesse trabalho, Paula et al. [2009] apresentam o método de influência local para modelos lineares com erros elípticos AR(1). Já Ibacache-Pulgar e Paula [2011] desenvolvem a metodologia para modelos parcialmente lineares com erros t de Student.

\subsection{Proposta de trabalho}

O principal objetivo desta dissertação é estudar os modelos parcialmente lineares com erros simétricos e estrutura de autocorrelação autoregressiva de primeira ordem. Neste capítulo faremos uma breve descrição dessa classe sob erros normais e apresentaremos um exemplo ilustrativo. No Capítulo 2 faremos uma breve revisão dos modelos não paramétricos, dos modelos simétricos parcialmente lineares e dos modelos de regressão com erros autoregressivos de ordem 1. No Capítulo 3 introduzimos a classe de modelos a ser estudada, derivamos algumas propriedades, as funções escore e matriz de informação de Fisher. Um processo iterativo para a estimação dos parâmetros é desenvolvido. No Capítulo 4 discutimos alguns procedimentos para a análise de diagnóstico em modelos parcialmente lineares com erros simétricos AR(1). No Capítulo 5 uma aplicação é apresentada e o Capítulo 6 apresenta as conclusões deste trabalho e eventuais pesquisas futuras. 


\subsection{Modelos parcialmente lineares com erros nor- mais $\operatorname{AR}(1)$}

Vamos considerar inicialmente o seguinte modelo de regresão:

$$
y_{i}=\mathbf{x}_{i}^{\top} \boldsymbol{\beta}+f\left(t_{i}\right)+\epsilon_{i},
$$

em que $\mathbf{x}_{i}=\left(x_{i 1}, \ldots, x_{i p}\right)^{\top}$ contém valores de variáveis explicativas, $\boldsymbol{\beta}=$ $\left(\beta_{1}, \ldots, \beta_{p}\right)^{\top}, \mathrm{f}($.$) é uma função não paramétrica, por exemplo um spline cú-$ bico, $t_{i}$ representa os valores de uma variável explicativa contínua que deve ser controlada de forma não paramétrica, $\epsilon_{i}$ são erros aleatórios tais que $\epsilon_{1}=e_{1}$ e $\epsilon_{i}=\rho \epsilon_{i-1}+e_{i}$ para $i=2, \ldots, n$ com $e_{i}$ independentes e identicamente distribuídos $N\left(0, \sigma^{2}\right)$. Podemos, alternativamente, escrever o modelo (1.1) na forma

$$
y_{i}=\mathbf{x}_{i}^{\top} \boldsymbol{\beta}+\boldsymbol{\eta}_{i}^{\top} \mathbf{f}+\epsilon_{i} .
$$

Este modelo, bem como sua generalização para erros simétricos, será discutido com maiores detalhes no Capítulo 3, onde serão apresentados suas propriedades, em quais situações a metodologia pode ser adequada e também será discutido um processo de estimação.

\subsection{Exemplo ilustrativo}

Nesta seção, apresentaremos um exemplo para facilitar o entendimento do modelo (1.1) apresentado acima. No Capítulo 5 voltaremos a analisar os dados utilizados neste exemplo ilustrativo.

Basicamente, tentaremos relacionar duas séries, com 120 observações, de temperaturas médias de duas cidades, Ubatuba e Cananéia. No Capítulo 5 descreveremos melhor onde é possível encontrar tais dados, bem como descreveremos melhor cada série. Esses dados representam temperaturas mensais médias de 1975 a 1985.

No Capítulo 5, para encontrarmos um modelo que satisfaça razoalvelmente as principais suposições impostas, desenvolveremos diversos modelos, partindo de um modelo simples, até obtermos o modelo final. Aqui, apresentaremos brevemente um possível modelo, que será discutido novamente no Capítulo 5.

O principal objetivo é relacionar a temperatura média de Ubatuba, variável resposta, com a variável explicativa, temperatura média de Cananéia, controlando pelo efeito do tempo, visto que a relação entre as séries pode sofrer 
alterações ao lonogo do tempo.

Portanto, considere o modelo (1.1) dado por

$$
y_{i}=\mathbf{x}_{i}^{\top} \boldsymbol{\beta}+\boldsymbol{\eta}_{i}^{\top} \mathbf{f}+\epsilon_{i}
$$

em que $\mathbf{x}_{i}$ é um vetor com um valor igual a 1, representando o intercepto, e com a $i$-ésima temperatura média de Cananéia, $y_{i}$ é a $i$-ésima temperatura média de Ubatuba, $f\left(t_{i}\right)$ uma função para controlar possíveis efeitos do tempo e $\epsilon_{i}$ são erros aleatórios tais que $\epsilon_{1}=e_{1}$ e $\epsilon_{i}=\rho \epsilon_{i-1}+e_{i}$, para $i=2, \ldots, n$, com $e_{i}$ independentes e identicamente distribuídos $N\left(0, \sigma^{2}\right)$. Através de um procedimento iterativo, discutido no Capítulo 3, maximizamos a verossimilhança dos dados e obtemos os parâmetros estimados desse modelo, que podem ser vistos na Tabela 1.1. Não apresentamos os valores ajustados da função não paramétrica devido à grande quantidade de efeitos estimados, que é igual ao tamanho amostral, no caso, 120.

O motivo da função não paramétrica apresentar essa quantidade de efeitos decorre do tipo de spline e penalização que estamos utilizando. Isso ficará mais claro ao longo deste trabalho, principalmente após a leitura do Capítulo 2.

Tabela 1.1: Estimativas dos parâmetros modelo (1.1) ajustado aos dados sobre temperaturas de Ubatuba e Cananéia.

\begin{tabular}{|r|r|r|r|r|}
\hline Efeito & Estimativa & Erro Padrão & Estatística t & Valor-p \\
\hline Intercepto & 4,645 & 0,075 & 61,913 & $<0,001$ \\
Cananéia & 0,825 & 0,003 & 239,65 & $<0,001$ \\
$\sigma^{2}$ & 0,568 & 0,007 & 84,853 & $<0,001$ \\
$\rho$ & 0,551 & 0,008 & 65,752 & $<0,001$ \\
\hline & & & & \\
\hline Graus de liberdade & 10,14 & & & \\
AIC & 295,8 & & & \\
\hline
\end{tabular}

Para realizar uma breve análise dos resíduos, construímos três gráficos, o gráfico normal de probabilidades com os resíduos do ajuste para verificar a suposição de normalidade e as funções de autocorrelação e autocorrelação parcial. Esses gráficos são descritos nas Figuras 1.1 e 1.2. 


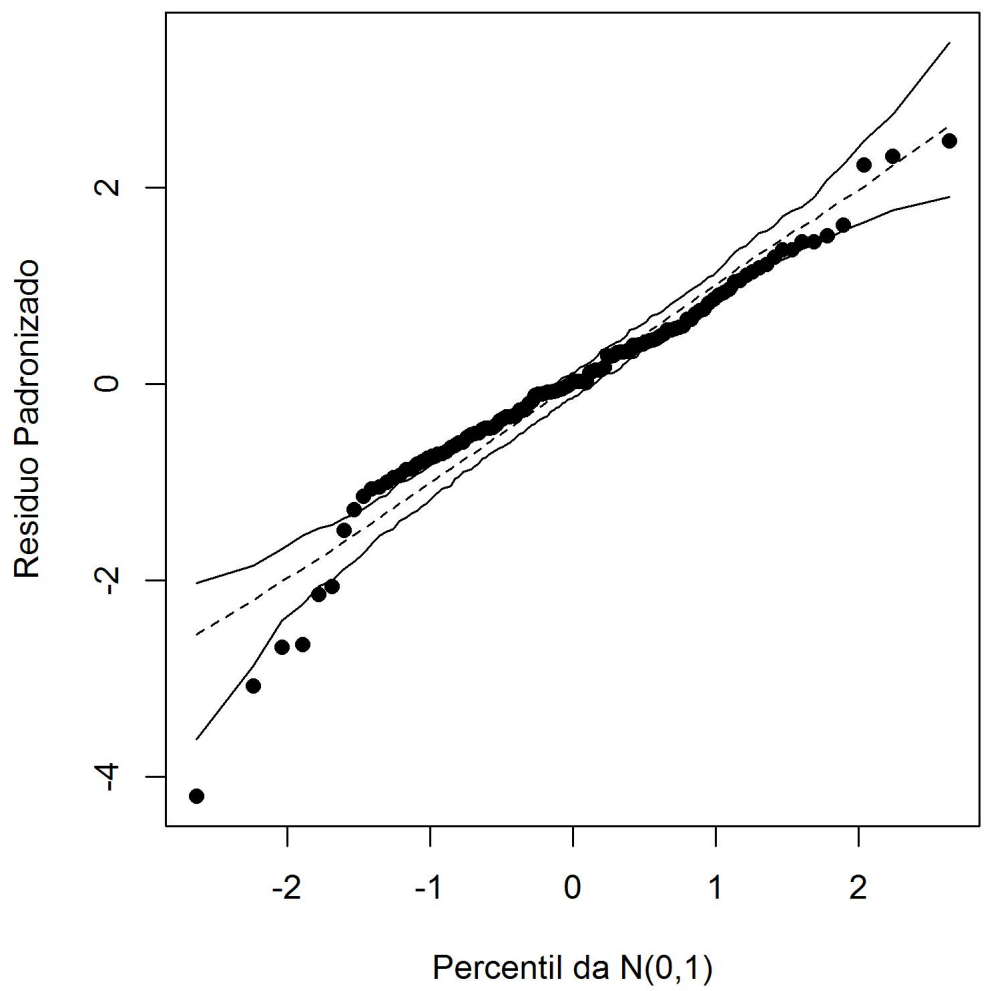

Figura 1.1: Gráfico normal de probabilidades para os resíduos padronizados referente ao modelo (1.1) ajustado aos dados sobre temperatura de Ubatuba e Cananéia. 
Função de autocorrelação dos resíduos padronizados

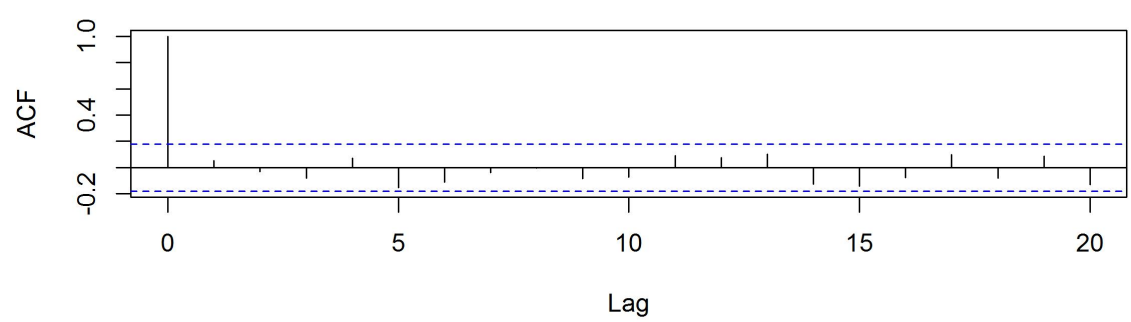

Função de autocorrelação parcial dos resíduos padronizados

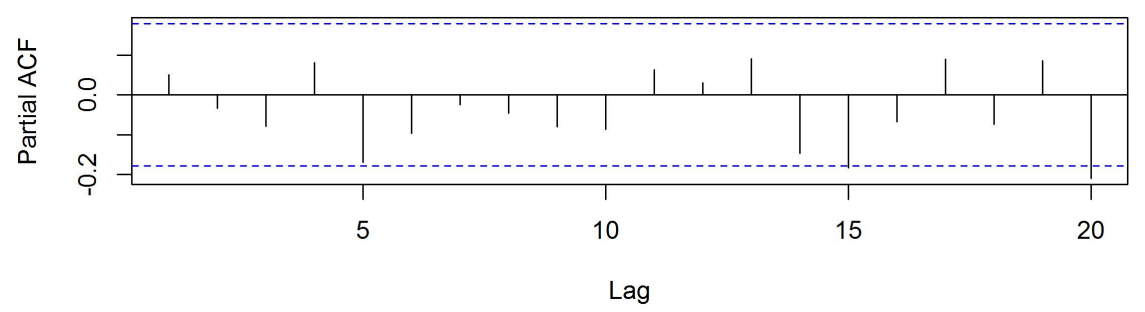

Figura 1.2: Funções de autocorrelação e autocorrelação parcial para os residuos do modelo (1.1) ajustado aos dados sobre temperaturas de Ubatuba e Cananéia.

Podemos notar pelos gráficos de autocorrelação e autocorrelação parcial que os resíduos não apresentam autocorrelação visto que em quase todos os lags analisados, essas funções são menores em valor absoluto do que a linha tracejada, limite de não rejeição de autocorrelação igual a 0.

No entanto, pelo gráfico normal de probabilidades (Figura 1.1), notamos que a distribuição dos resíduos apresenta mais valores altos, em módulo, do que seria esperado caso a distribuição fosse realmente normal, visto que os pontos nas caudas da distribuição estão fora da banda de confiança de $95 \%$, construída supondo normalidade. Vale ressaltar que tanto valores pequenos quanto altos destoam da distribuição normal, evidenciando que apesar de não parecer normal, a distribuição dos resíduos é relativamente simétrica. Portanto, o uso de uma distribuição simétrica com caudas mais pesadas pode se ajustar melhor aos dados, produzindo inferências mais confiáveis.

A banda de confiança apresentada na Figura 1.1 é conhecida na literatura como envelope e é construída através de simulações, neste caso, da distribuição normal. Maiores detalhes de envelope podem ser encontrados em Paula [2010]. 
Esse trabalho tem como objetivo generalizar o modelo (1.1) para abranger as distribuições simétricas. 


\section{Capítulo 2}

\section{Revisão de modelos semiparamétricos autogressivos}

Neste capítulo, apresentaremos uma breve descrição dos modelos semiparamétricos, do modelo normal linear com erros autoregressivos de ordem $1 \mathrm{e}$ intoduziremos, também, os modelos parcialmente lineares com erros simétricos. O objetivo deste capítulo é familiarizar o leitor com esses modelos, com o intuito de facilitar o entendimento dos capítulos posteriores e consequentemente o modelo proposto neste trabalho, que também envolve erros simétricos, uma parte não paramétrica e erros autoregressivos de primeira ordem. Este capítulo está dividido da seguinte forma:

- A Seção 2.1 tem como objetivo apresentar os modelos semiparamétricos. Inicialmente, apresentamos os modelos não paramétricos para depois abrangermos os modelos semiparamétricos.

- Já a Seção 2.2 traz os modelos parcialmente lineares com erros simétricos, em que a suposição de normalidade dos erros no modelo definido na Seção 2.1 será generalizada para distribuições simétricas.

- Por fim, a Seção 2.3 abrange o modelo normal linear com erros AR(1), onde apresentaremos procedimentos de estimação frequentemente utilizados.

\subsection{Modelos semiparamétricos}

Nesta seção, introduzimos os modelos semiparamétricos, que são modelos compostos basicamente por uma parte não paramétrica e uma parte paramé- 
trica. Por isso, inicialmente, definiremos os modelos não paramétricos na Subseção 2.1.1. Após o entedimento dos modelos não paramétricos, abrangeremos os modelos semiparamétricos. Boas referências para o estudo desses modelos são Ruppert et al. [2003], Ruppert [2004] e Green e Silverman [1994].

\subsubsection{Modelos não paramétricos}

Modelos de regressão lineares são utilizados quando é conhecido que as relações entre as variáveis preditoras e a variável resposta é linear. Quando as relações entre as variáveis são não lineares, porém conhecidas, uma alternativa aos modelos lineares são os modelos de regressão não linear. No entanto, há situações em que a relação entre as variáveis não é linear, nem tampouco conhecida. Assim, surgiram os modelos não paramétricos, em que não há a necessidade de prefixar a relação a ser utilizada no modelo.

Existem diversas técnicas de regressão não paramétricas. Neste trabalho, abordaremos inicialmente a técnica apresentada em Ruppert et al. [2003], que é a regressão não paramétrica através de splines. Splines são funções construídas por meio da junção de várias funções polinomiais.

Vale ressaltar que os modelos não paramétricos devem ser utilizados apenas quando há muita informação contida nos dados para podermos estimar a relação entre as variáveis de forma consistente, visto que esta relação não é pré-fixada. Muita informação significa que há muitas observações, que há pouco ruído nos dados ou que as variáveis preditoras variam em praticamente todo o espaço.

Iremos iniciar a construção de splines através de splines lineares. Após a descrição desses splines, iremos abordar splines quadráticos e de ordem maior. Um spline linear é definido como a junção de várias funções lineares em determinados pontos, chamados de nós.

Vamos considerar inicialmente o caso em que há apenas um nó. Neste caso, o spline pode ser definido como $s(x)=a+b x$ se $\mathrm{x}<\mathrm{t}$ e $s(x)=c+d x$ para $\mathrm{x}$ $>$ t. Temos que deve valer a igualdade $a+b t=c+d t$, pois devem ser iguais para $x=t$. Resolvendo em função de c, podemos escrever o spline como

$s(x)= \begin{cases}a+b x, & x<t, \\ a+b x+(d-b)(x-t), & x \geq t .\end{cases}$

Para facilitar o desenvolvimento da teoria, é utilizada a seguinte notação: $(x-t)_{+}= \begin{cases}0, & x<t \\ (x-t), & x \geq t .\end{cases}$ 
Usando esta notação, podemos reescrever o spline como

$$
s(x)=a+b x+(d-b)(x-t)_{+} .
$$

Vamos agora estender esse resultado para o caso em que temos mais de um nó. Vamos supor que temos $k$ nós denotados por $t_{1}, \ldots, t_{k}$. Portanto, utilizando-se da notação anterior, podemos construir o spline com múltiplos nós como

$$
s(x)=\eta_{0}+\eta_{1} x+b_{1}\left(x-t_{1}\right)_{+}+b_{2}\left(x-t_{2}\right)_{+}+\cdots+b_{k}\left(x-t_{k}\right)_{+} .
$$

Vale destacar que o spline construído é composto por funções contínuas, logo também é contínuo. Observamos, ainda, que $b_{j}$ indica o quanto a primeira derivada do spline varia quando $x$ se torna maior ou igual ao $j$-ésimo nó.

Os splines lineares apresentam uma espécie de "bico", na função, em cada nó. Caso não se deseje que a curva estimada apresente esses "bicos", podem ser definidos splines de ordem maior. Por exemplo, podemos definir um spline quadrático. Esse spline é dado por

$$
s(x)=\eta_{0}+\eta_{1} x+\eta_{2} x^{2}+b_{1}\left(x-t_{1}\right)_{+}^{2}+b_{2}\left(x-t_{2}\right)_{+}^{2}+\cdots+b_{k}\left(x-t_{k}\right)_{+}^{2}
$$

em que,

$$
(x-t)_{+}^{2}= \begin{cases}0, & x<t \\ (x-t)^{2}, & x \geq t\end{cases}
$$

Se $x \geq t$, temos que a primeira derivada de $(x-t)_{+}^{2}$ é igual a $2(x-t)$. Já a segunda derivada é igual a 2. Quando $\mathrm{x}<\mathrm{t}$, as duas derivadas são iguais a 0. Portanto, a curva do spline quadrático estimado não apresenta mais os "bicos"comentados anteriormente.

De forma geral, podemos definir um spline de ordem $p$. Esse spline é dado por

$$
s(x)=\eta_{0}+\eta_{1} x+\cdots+\eta_{p} x^{p}+b_{1}\left(x-t_{1}\right)_{+}^{p}+b_{2}\left(x-t_{2}\right)_{+}^{p}+\cdots+b_{k}\left(x-t_{k}\right)_{+}^{p} .
$$

Na prática, costuma-se usar splines lineares ou quadráticos, até mesmo cúbicos. Não é muito utilizado splines de ordens maiores do que 3. A literatura 
da área sugere que não há muitas diferenças ao utilizarmos um spline de ordem maior do que 2 ou 3.

Os parâmetros do spline que devem ser estimados são: $\eta_{0}, \eta_{1}, \ldots, \eta_{p}, b_{1}, \ldots$, $b_{k}$. Esses parâmetros podem ser estimados através do método de mínimos quadrados.

Para visualizar melhor as diferenças entre os possíveis graus dos polinômios utilizados, construímos para a série de temperatura de Ubatuba, discutida no primeiro capítulo, um spline da série contra o tempo. Abaixo, podemos ver três ajustes diferentes, considerando polinômios lineares, quadráticos e cúbicos. Assim, fica mais claro os comentários anteriores.
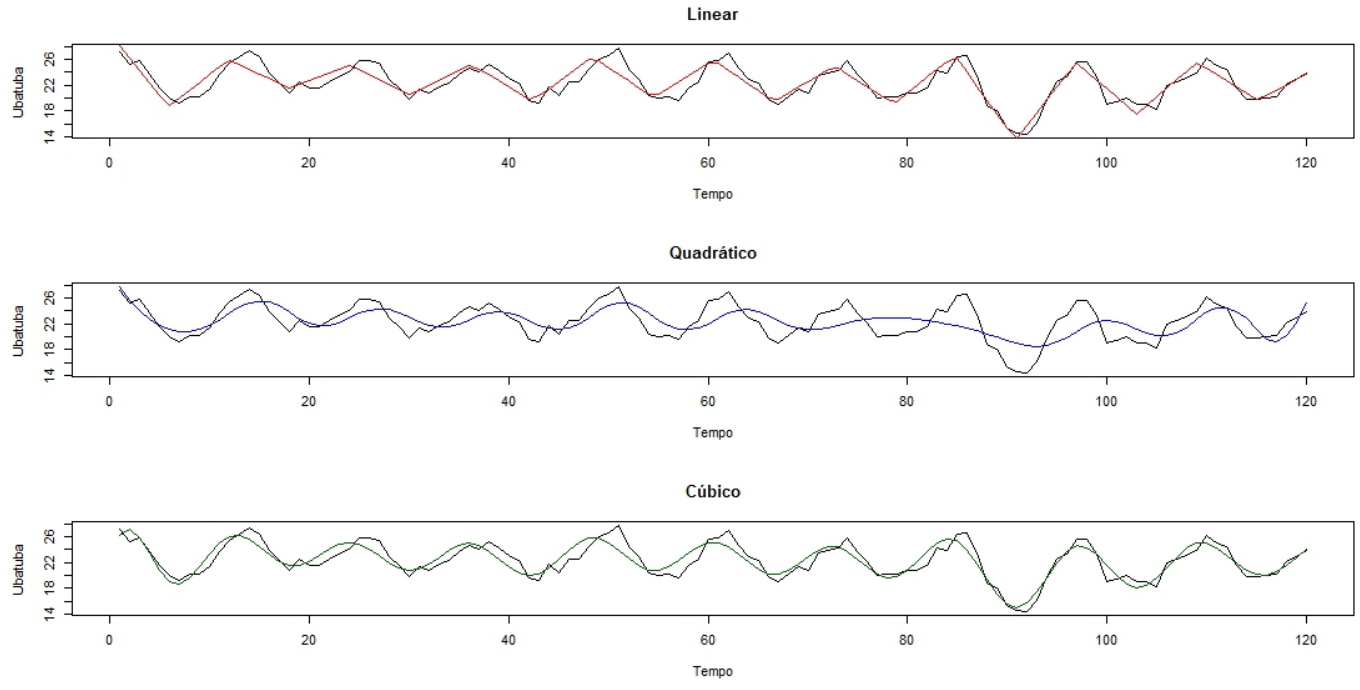

Figura 2.1: Splines estimados, para a temperatura de Ubatuba ao longo do tempo, considerando polinômios lineares, quadráticos e cúbicos

Ruppert et al. [2003] apresenta três questões que são geralmente colocadas ao estudarem pela primeira vez esse tipo de modelo. São elas,

- Qual o grau de spline deve ser utilizado?

- Quantos nós devem ser escolhidos?

- Onde esses nós devem ser colocados? 
Na primeira questão, ele sugere utilizar splines lineares ou quadráticos, com as vantagens do quadrático já comentadas anteriormente. Quanto à posição em que os nós devem ser colocados, é sugerido que se coloque os nós igualmente espaçados.

Já a segunda questão é considerada a mais polêmica e de mais difícil resposta. É conhecido na literatura de modelos não paramétricos que o resultado da reta estimada é bastante sensível ao número de nós escolhidos na estimação de mínimos quadrados. No entanto, há várias técnicas para tentar reduzir esta sensibilidade na curva estimada. Neste capítulo introdutório, mostraremos apenas a técnica que produz um determinado tipo de spline, os chamados splines penalizados. Caso seja de interesse o conhecimento de outras técnicas, sugerimos as referências aqui utilizadas e específicas.

A teoria apresentada até aqui utiliza apenas uma variável preditora. No entanto, sem muitos problemas, conseguimos acrescentar outras variáveis no modelo de forma aditiva, isto é, sem incluir interação. É possível, também, incluir esta interação, porém não será objeto de estudo neste trabalho.

Ao estimarmos os parâmetros dos splines por mínimos quadrados, a escolha do número de nós utilizados é ainda mais importante. Se escolhermos um número insuficiente de nós, a reta estimada pode não ser bem ajustada. Por outro lado, se escolhermos um número muito grande de nós, obteremos um super ajustamento, o que também não é adequado. Uma possibilidade para resolver este problema seria escolher o número de nós através de algum critério de seleção de modelo, como por exemplo AIC, BIC ou $C_{p}$.

No entanto, a técnica de splines penalizados também é bastante útil para a escolha do número de nós. Logo, apresentaremos esta técnica, visto que será utilizada nos capítulos posteriores.

Basicamente, o método de splines penalizados consiste na utilização de um número grande, porém arbitrário, de nós e ao invés de utilizarmos estimação por mínimos quadrados, utilizamos um método de estimação que controla o super ajustamento. Portanto, com este método, não é necessária a escolha do número ideal de nós.

Um super ajustamento ocorre quando há muitos saltos na reta ajustada ou quando os saltos não são limitados, ou seja, quando a reta estimada sofre diversas alterações. Portanto, podemos evitar um super ajustamento evitando um desses fatos. A penalização limita o tamanho do salto e, por consequência, evita esse super ajustamento.

O tamanho do $k$-ésimo salto para os splines quadráticos, por exemplo, é proporcional a $b_{k}$. Logo, a soma de todos os saltos ao quadrado, considerando 
$K$ nós, é proporcional a

$$
\sum_{k=1}^{K} b_{k}^{2}
$$

Uma maneira de evitar super ajustamento é limitarmos a quantidade acima. Isto pode ser realizado ao mesmo tempo que minimizamos o erro quadrático do ajuste. Portanto, podemos minimizar a seguinte função:

$$
\begin{array}{r}
\sum_{i=1}^{n}\left\{y_{i}-\left(\eta_{0}+\eta_{1} x+\eta_{2} x^{2}+b_{1}\left(x-t_{1}\right)_{+}^{2}+b_{2}\left(x-t_{2}\right)_{+}^{2}+\cdots+b_{K}\left(x-t_{K}\right)_{+}^{2}\right)\right\}^{2} \\
+\alpha \sum_{k=1}^{K} b_{k}^{2} .
\end{array}
$$

O parâmetro $\alpha$ representa o peso dos saltos ao quadrado na equação acima. Este peso é conhecido como parâmetro de penalização ou de suavização, podendo variar de 0 a $\infty$. A escolha deste peso é muito importante no processo de estimação. Se escolhermos $\alpha$ igual a 0 , teremos uma estimação de mínimos quadrados. Por outro lado, à proporção que aumentamos o valor de $\alpha$, os saltos vão se aproximando de 0 a fim de minimizar a equação acima, ou seja, os parâmetros $b_{0}, b_{1}, \ldots, b_{K}$ irão se concentrar em torno do valor nulo. Com isso, o spline se aproximar-se-á de um simples polinômio quadrático, podendo ser representado por

$$
s(x)=\eta_{0}+\eta_{1} x+\eta_{2} x^{2} .
$$

É importante ressaltar que esta penalização pode variar dependendo do grau do polinômio utilizado. A penalização acima é utilizada para splines quadráticos. Caso passemos a trabalhar com outros graus, poderemos construir a penalização da mesma forma.

A escolha do valor de $\alpha$ é muito importante, visto que o ajuste é sensível a diferentes valores escolhidos. Para visualizarmos essas diferenças, na Figura 2.2 mostramos o ajuste da série de temperaturas de Ubatuba com diferentes valores de $\alpha$, evidenciando tais diferenças. 

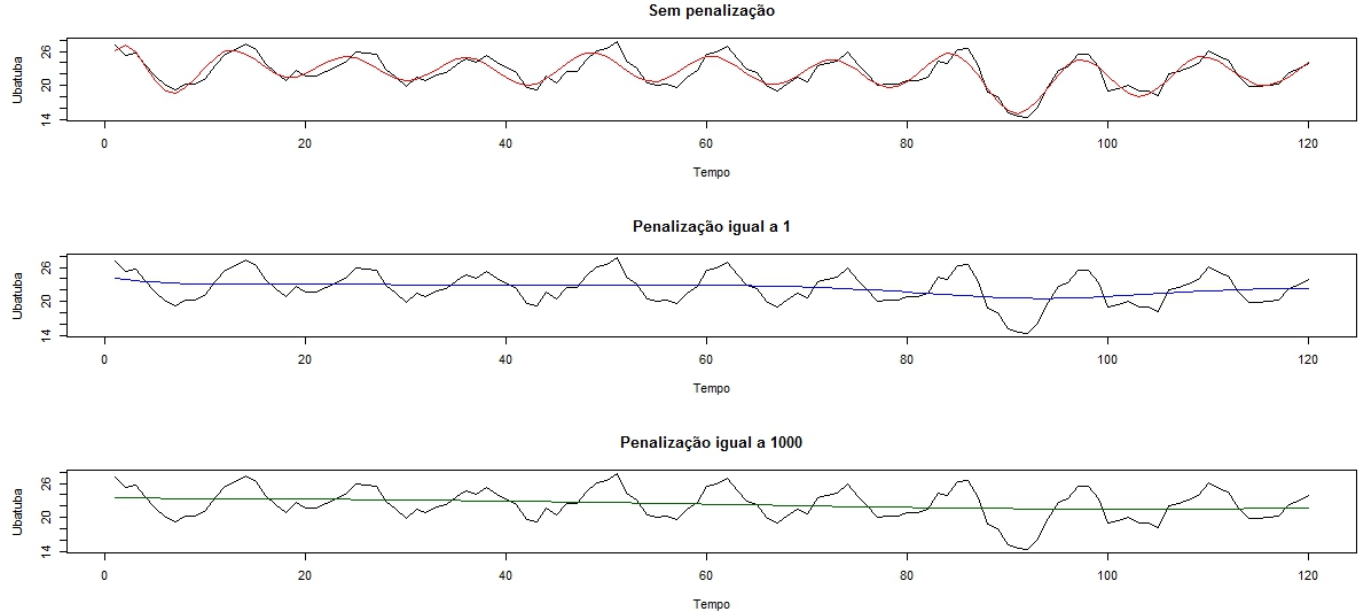

Figura 2.2: Diferentes valores do parâmetro de suavização - Temperatura de Ubatuba.

Por ser crucial no processo de estimação, há algumas técnicas na literatura especializada para uma boa escolha de $\alpha$. A técnica que talvez seja a mais conhecida é a validação cruzada em que a escolha do melhor valor do parâmetro de suavização é o valor que nos fornece as melhores predições. De forma geral, o procedimento de validação cruzada é dado por:

- Primeiramente, devemos fixar um valor de $\alpha$. Após, eliminamos a primeira observação e estimamos o modelo sem esta observação. Então, predizemos a observação que foi retirada e calculamos o erro de predição.

- Repetimos o prodecimento anterior para todas as observações presentes no modelo.

- Com todos os erros de predição, podemos encontrar o erro de predição quadrático médio.

- Alteramos o valor de $\alpha$ e repetimos todos os itens anteriores.

Pela técnica de validação cruzada, escolhemos o valor de $\alpha$ que minimiza o erro de predição quadrático médio.

A validação cruzada apresenta um elevado custo computacional, por esta razão, foi proposta uma aproximação a esta medida, sendo conhecida como 
validação cruzada generalizada. Mais informações podem ser encontradas em Ruppert et al. [2003].

O último tópico referente a modelos não paramétricos apresentados neste trabalho é como obter o número efetivo de parâmetros. Por exemplo, como já discutimos anteriormente, quando $\alpha$ cresce, os parâmetros $b_{0}, b_{1}, \ldots, b_{K}$ se aproximam de 0 . Logo, neste caso, o número de parâmetros não é igual a $1+p+K$, que seria o número de parâmetros caso todos fossem diferentes de 0 , equivalente a $\alpha$ ser igual a 0 . Quando $\alpha$ é igual a $\infty$, o caso extremo, todos os parâmetros $b^{\prime}$ s seriam iguais a 0 e portanto o número de parâmetros seria igual a $1+p$. Portanto, é fácil visualizar que no caso em que $\alpha$ é um número positivo, porém finito, o número de parâmetros está entre os valores comentados neste parágrafo. Adiante, ainda neste capítulo, apresentaremos a fórmula para a obtenção dos graus de liberdade efetivos para um caso particular.

A forma como utilizamos o componente não paramétrico, até o momento, é talvez a forma mais fácil de compreender o conceito dos splines. No entanto, neste trabalho, utilizaremos a forma não paramétrica de uma outra maneira, como apresentada em Green e Silverman [1994].

Os autores definem o componente não paramétrico de tal forma que entre dois nós consecutivos exista um spline cúbico natural e que para $t<t_{1}$ e para $t>t_{n}$, o componente não paramétrico seja linear. Também, é sugerido utilizar todos os pontos distintos da variável como nós. Já a respeito da penalização, utilizamos penalizações do tipo $J(f)=\int_{a}^{b}\left[f^{l}(t)\right]^{2} \mathrm{~d} t$, em que $a$ e $b$ são os limites onde a função varia, ou seja, $a<t_{1}$ e $b>t_{n}$ e $f^{l}(t)=\frac{\mathrm{d}^{l}}{\mathrm{~d} t^{l}} f(t)$.

Consideremos que $t_{1}^{0}, \ldots, t_{q}^{0}$, os valores ordenados e distintos de $t_{1}, \ldots, t_{n}$, são os nós utilizados para a parte não paramétrica, isto é, temos $q$ nós. A função $f$ pertence às funções do espaço de Sobolev, definido como

$\mathcal{W}_{2}^{(l)}=\left\{f: f, f^{(1)}, \ldots, f^{(l-1)}\right.$ funções absolutamente contínuas, $\left.f^{(l)} \in \mathcal{L}^{2}[a, b]\right\}$.

É possível mostrar que para $l=2$, a estimação de $f$ gera um spline cúbico natural com nós nos pontos $t_{1}^{0}, \ldots, t_{q}^{0}$. De acordo com Green e Silverman [1994] podemos expressar $J(f)$ como $\mathbf{f}^{\top} \mathbf{K} \mathbf{f}$. A matriz $\mathbf{K}$ é uma matriz $q \times q$ que depende dos nós e do grau utilizado. Mais especificamente, $\mathbf{K}=\mathbf{Q R}^{-1} \mathbf{Q}^{\top}$, em que $\mathbf{Q}$ é uma matriz $q \times(q-2)$ e $\mathbf{R}$ é uma matriz $(q-2) \times(q-2)$. Os valores $q_{i j}$ para $i=1, \ldots, q$ e $j=2, \ldots,(q-1)$ são dados por

$$
q_{(j-1) i}=h_{j-1}^{(-1)}, q_{j j}=h_{j-1}^{(-1)}-h_{j}^{(-1)}, \quad \text { e } q_{(j+1) j}=h_{j}^{(-1)}
$$


para $j=2, \ldots, q-1$ e igual a 0 caso contrário. Os elementos de $\mathbf{R}$ para $i$ e $j$ variando de 2 a $n-1$ são iguais a

$$
r_{i i}=\frac{1}{3}\left(h_{i-1}+h_{i}\right), r_{i(i+1)}=r_{(i+1) i}=\frac{1}{6} h_{i} .
$$

Os elementos $h_{i}$ são dados por $t_{i+1}^{0}-t_{i}^{0}$ para $i$ de 1 a $q$.

Maiores detalhes sobre essa construção podem ser vistos em Green e Silverman [1994].

A notação utilizada por nós neste trabalho, para a parte não paramétrica, é a mesma utilizada no livro. Portanto denotamos que $f(t)=\mathbf{N f}$ em que $\mathbf{f}=\left(f_{1}, \ldots, f_{q}\right)^{\top}$. Já $\mathbf{N}$ é uma matriz $q \times q$ chamada de matriz de incidência, formada por zeros e uns, dependendo dos valores de $t_{1}^{0}, \ldots, t_{q}^{0}$. Por exemplo, se todos os valores de $t$ forem distintos e ordenados, a matriz $\mathbf{N}$ será a matriz identidade.

\subsubsection{Modelos semiparamétricos}

Neste capítulo, abrangemos os modelos semiparamétricos, considerando somente uma variável não paramétrica. A extensão para casos aditivos, ou seja, com mais efeitos não paramétricos não é difícil.

Ruppert et al. [2003] apresenta os modelos semiparamétricos por meio de modelos de efeitos mistos, no entanto, não utilizaremos esta abordagem nos capítulos posteriores.

Para apresentarmos os modelos semiparamétricos neste trabalho, apenas estenderemos o modelo não paramétrico apresentado na seção anterior, incluindo preditores utilizados na forma paramétrica. Neste caso, podemos definir o modelo semiparamétrico utilizando um spline na variável aleatória $t$ com $k$ raízes e com preditores paramétricos $x_{1}, \ldots, x_{j}$, da seguinte maneira:

$$
\begin{aligned}
& y_{i}=\eta_{0}+\eta_{1} t_{i}+\cdots+\eta_{p} t_{i}^{p}+b_{1}\left(t_{i}-t_{1}\right)_{+}^{p}+b_{2}\left(t_{i}-t_{2}\right)_{+}^{p}+\cdots+b_{k}\left(t_{i}-t_{k}\right)_{+}^{p}+ \\
& +\beta_{1} x_{i 1}+\cdots+\beta_{j} x_{i j}+\epsilon_{i} .
\end{aligned}
$$

No modelo acima, consideramos em um primeiro momento que a variável aleatória $\epsilon_{i}$ apresenta uma distribuição normal com média 0 e variância $\sigma^{2}$, no entanto, a estimação por mínimos quadrados não requer esta suposição. Vale ressaltar que o vetor de parâmetros $\boldsymbol{\beta}$ é interpretado da mesma maneira que em modelos de regressão linear. Uma única ressalva é que, além de todos os efeitos paramétricos serem mantidos constantes para a interpretação, o efeito não pa- 
ramétrico, a variável $t$, também deve ser mantida constante. Já os parâmetros $\eta$ 's e os parâmetros b's são de difícil interpretação. Uma possível maneira para interpretar a parte não paramétrica é fixar os efeitos paramétricos e construir o gráfico da curva estimada para todo o horizonte em que a variável $t$ atua. Com isso, podemos visualizar o comportamento da variável $t$ no espaço em que ela se concentra.

O efeito não paramétrico, por apresentar um número maior de parâmetros, geralmente, leva a melhores resultados. No entanto, como salientamos anteriormente, esse efeito é de difícil interpretação. Portanto, usualmente, o efeito não paramétrico é utilizado quando a relação entre as variáveis não é conhecida e/ou quando queremos controlar o efeito de forma mais rigorosa, sem termos a necessidade de interpretar o efeito dessa variável.

A estimação dos parâmetros do modelo se faz da mesma forma que nos modelos não paramétricos, ou seja, por meio do método de mínimos quadrados, utilizando-se da função de penalização. Podemos encontrar o mínimo da função apresentada para o caso não paramétrico, apenas acrescentando os termos dos efeitos paramétricos.

O mesmo raciocínio pode ser utilizado para a função não paramétrica na definição presente em Green e Silverman [1994]. Nesse caso, o modelo semiparamétrico é, seguindo a notação usual, dado por

$$
\mathrm{y}=\mathrm{X} \boldsymbol{\beta}+\mathrm{Nf}+\boldsymbol{\epsilon}
$$

em que $\mathbf{X}$ é a matriz modelo contendo as variáveis explicativas que contribuem de maneira paramétrica sob a resposta, $\mathbf{N}$ é novamente uma matriz de incidência de zeros e uns que dependem dos valores da variável não paramétrica, $\boldsymbol{\beta}$ e f são os parâmetros a serem estimados e $\boldsymbol{\epsilon}$ representa o vetor de erros do modelo.

Os parâmetros do modelo acima podem ser estimados por meio de mínimos quadrados penalizados ou por máxima verossimilhança penalizada, supondo alguma distribuição para $\boldsymbol{\epsilon}$. A penalização é definida da mesma forma que em modelos não paramétricos e a estimação da função $f$ é um spline cúbico natural. 


\subsection{Modelos parcialmente lineares com erros si- métricos}

Nesta seção, realizamos uma breve descrição dos modelos parcialmente lineares com erros simétricos. Para tanto, seguimos o artigo de Ibacache-Pulgar et al. [2012]. Os autores apresentam essa classe de modelos generalizando para $s$ funções não paramétricas. Aqui, apresentamo-la supondo apenas uma função não paramétrica.

O modelo definido nesta seção, basicamente abrange o modelo definido na Seção 2.1, visto que apresenta uma função não paramétrica e uma parte paramétrica. No entanto, o modelo presente expande as suposições possíveis para a distribuição dos erros, podendo pertencer à classe de distribuições simétricas.

Antes de definirmos o modelo, realizaremos uma breve descrição das distribuições simétricas e dos modelos de regressão com erros simétricos.

\subsubsection{Distribuições simétricas univariadas}

Tradicionalmente, os modelos supondo a normalidade dos erros sempre foram os mais utilizados devido à maior facilidade de entendimento e maior facilidade computacional. No entanto, esta suposição nem sempre é verdadeira, podendo haver indícios de simetria nos dados porém com caudas mais pesadas ou mais leves do que as caudas da distribuição normal. Outra deficiência do modelo com erros normais é que as estimativas de mínimos quadrados podem ser altamente sensíveis a observações aberrantes. Para contornar esses problemas, várias alternativas foram propostas no decorrer dos anos. Inicialmente, foram propostas as conhecidas transformações nos dados para alcançar a normalidade, porém há situações em que essas transformações não produzem resultados satisfatórios. Devido a tal fato, também surgiram métodos robustos (Huber [1973]) para tais observações aberrantes. Foi proposto também aumentar o leque de possíveis distribuições para os erros, como por exemplo, considerar distribuições que apresentem caudas mais pesadas ou mais leves do que a normal e que sejam simétricas, assim como a normal. Essas distribuições são conhecidas como simétricas.

A maioria das distribuições simétricas apresentam, além de parâmetros de posição e escala, como a média e a variância, parâmetros de forma, que controlam a curtose da distribuição. Em particular, o parâmetro de curtose fornece uma maior flexibilidade nas caudas dessas distribuições, podendo levar a ajustes mais adequados. Por exemplo, para a distribuição t de Student, o 
número de graus de liberdade controla a curtose e, portanto, o peso das caudas.

Não apresentamos, com detalhes, todos os resultados de modelos com erros simétricos. Caso seja do interesse do leitor, sugerimos Cysneiros [2004], Fang et al. [1990] e Fang e Anderson [1990].

Há várias distribuições de probabilidade pertencentes à classe de distribuições simétricas, entre elas podemos destacar a normal, normal contaminada, $t$ de Student, t de Student generalizada, logísticas tipo I e tipo II, dentre outras.

De Cysneiros [2004], formalmente, podemos definir uma distribuição sendo simétrica como:

\section{Definição 1:}

Seja a variável aleatória y com suporte em $\mathbb{R}$, com parâmetros de locação $\mu \in$ $\mathbb{R}$ e de escala $\phi>0$ com função de densidade de probabilidade dada por:

$$
f(y ; \mu, \phi)=\frac{1}{\sqrt{\phi}} g\left[\frac{(y-\mu)^{2}}{\phi}\right], \quad y \in \mathbb{R}
$$

para alguma função $g($.$) denominada função geradora de densidades, com g(\mu)$ $>0$, para $\mu>0$ e $\int_{0}^{\infty} u^{-\frac{1}{2}} g(u) d u=1$. Esta condição é necessária para que $f(y ; \mu, \phi)$ seja uma função densidade de probabilidade. Denotamos por y $\sim$ $\mathrm{S}(\mu, \phi)$ e denominamos de variável aleatória simétrica.

Pela definição acima, podemos observar que a função $\mathrm{g}($.$) caracteriza a$ função de probabilidade simétrica.

Vamos, agora, apresentar algumas propriedades destas distribuições. Seja $\mathrm{y} \sim \mathrm{S}(\mu, \phi)$, temos que

- $\mathrm{E}[\mathrm{y}]=\mu ;$

- $\operatorname{Var}[y]=\xi \phi$, em que $\xi>0$ é uma constante que depende da distribuição simétrica que está sendo utilizada;

- Se y $\sim \mathrm{S}(\mu, \phi)$, então a + by $\sim \mathrm{S}\left(a+b \mu, b^{2} \phi\right)$, em que a, b $\in \mathbb{R}$ com $\mathrm{b} \neq 0$. Logo, a combinação linear de uma variável aleatória simétrica é também uma variável aleatória simétrica. Uma padronização que será utilizada no decorrer deste trabalho é a seguinte:

$$
z=\frac{(y-\mu)}{\sqrt{\phi}} \sim S(0,1) .
$$




\subsubsection{Modelos de regressão linear com erros simétricos}

Sejam $\epsilon_{1}, \ldots, \epsilon_{n}$ variáveis aleatórias independentes com distribuições pertencentes à classe de distribuições simétricas. Logo,

$$
f_{\epsilon_{i}}(\epsilon)=\frac{1}{\sqrt{\phi}} g\left[\frac{\epsilon^{2}}{\phi}\right] .
$$

O modelo de regressão simétrico linear é definido por,

$$
y_{i}=\mathbf{x}_{i}^{\top} \boldsymbol{\beta}+\epsilon_{i}, \quad i=1, \ldots, n,
$$

em que $\mathbf{x}_{i}$ representa o vetor de variáveis explicativas para o $i$-ésimo indíviduo, $\boldsymbol{\beta}$ é um vetor de parâmetros $p \times 1$ e $\epsilon_{i}$ segue uma distribuição simétrica.

Pelas propriedades das ditribuições simétricas comentadas na subseção anterior, temos que a função densidade de $y_{i}$ é dada por

$$
f_{y_{i}}\left(y_{i}\right)=\frac{1}{\sqrt{\phi}} g\left(u_{i}\right)
$$

em que

$$
u_{i}=\frac{\left(y_{i}-\mu_{i}\right)^{2}}{\phi}, \quad \mu_{i}=\mathbf{x}_{i}^{\top} \boldsymbol{\beta} \quad \text { e } \quad y_{i} \sim S\left(\mu_{i}, \phi\right) .
$$

O modelo definido acima é conhecido como modelo simétrico linear. Poderíamos facilmennte estender este modelo para abranger casos não lineares. Temos que o vetor de parâmetros $\boldsymbol{\theta}$ neste caso é composto pelos vetores $\boldsymbol{\beta}$ e pelo parâmetro de dispersão $\phi$, isto é $\boldsymbol{\theta}=\left(\boldsymbol{\beta}^{\top}, \phi\right)^{\top}$.

Por meio das distribuições dos $y_{i}^{\prime} s$, temos que o logaritmo da função de verossimilhança de $\boldsymbol{\theta}$ pode ser expresso na forma

$$
L(\boldsymbol{\theta})=-\frac{n}{2} \log \phi+\sum_{i=1}^{n} \log \left\{g\left(u_{i}\right)\right\} .
$$

A estimação dos parâmetros é feita maximizando a verossimilhança acima através de métodos computacionais usuais, como Newton-Raphson, escore de Fisher, entre outros.

\subsubsection{Modelo parcialmente linear com erros simétricos}

Através das discussões apresentadas na Seção 2.1 e nas subseções anteriores, notamos que o modelo parcialmente linear com erros simétricos é útil 
quando a relação da variável resposta com a variável explicativa não é linear nem tampouco conhecida, podendo ser usada de maneira não paramétrica. Além disso, podemos utilizar uma distribuição com caudas mais pesadas, diminuindo, assim, o peso de observações aberrantes nas estimativas do modelo, produzindo assim ajustes mais robustos.

Os modelos parcialmente lineares com erros simétricos são definidos da seguinte forma:

$$
y_{i}=\mathbf{x}_{i}^{\top} \boldsymbol{\beta}+f\left(t_{i}\right)+\epsilon_{i}
$$

com $i=1, \ldots, n$, e $y_{i}$ a $i$-ésima variável resposta, $\mathbf{x}_{i}^{\top}$ o vetor $(p \times 1)$ de valores das variáveis explicativas, $f($.$) uma função de uma variável explicativa$ que contribui de maneira não paramétrica e $\epsilon_{i}$ representa o termo do erro da $i$-ésima observação, em que assumimos que $\epsilon_{i}$ apresenta uma distribuição simétrica, ou seja, $\epsilon_{i} \sim S(0, \phi)$. Este modelo, escrito em forma matricial, pode ser representado por

$$
\mathbf{y}=\mathbf{X} \boldsymbol{\beta}+\mathbf{N f}+\boldsymbol{\epsilon}
$$

em que $\mathbf{y}$ é um vetor $(n \times 1)$, $\mathbf{X}$ é uma matriz $(n \times p)$ com cada linha igual a $\mathbf{x}_{i}^{\top}, \mathbf{N}$ é uma matriz $(n \times q)$ de zeros e uns em que $q$ denota os valores distintos de $t_{i}$, sendo denominada de matriz de incidência.

A função de verossimilhança do modelo definido acima pode ser escrito da seguinte forma:

$$
L(\boldsymbol{\theta})=-\frac{n}{2} \log \phi+\sum_{i=1}^{n} \log \left\{g\left(\frac{\left(y_{i}-\mathbf{x}_{i}^{\top} \boldsymbol{\beta}-f\left(t_{i}\right)\right)^{2}}{\phi}\right)\right\},
$$

em que $\boldsymbol{\theta}=\left(\boldsymbol{\beta}^{\top}, \mathbf{f}^{\top}, \phi\right)^{\top}$. Conforme salientamos na Seção 2.1, a utilização da verossimilhança penalizada pode reduzir a presença de super ajuste na presença de um número elevado de nós. Da mesma forma, define-se o logaritmo da função de verossimilhança penalizada como

$$
L_{\mathrm{p}}(\boldsymbol{\theta}, \alpha)=L(\boldsymbol{\theta})-\frac{\alpha}{2} \mathbf{f}^{\top} \mathbf{K} \mathbf{f},
$$

em que, novamente, $\alpha$ é o parâmetro de suavização e $\mathbf{K}$ é a matriz de penalização $(q \times q)$ definida da mesma maneira que anteriormente.

Nas próximas subseções, apresentaremos as funções escore e as matrizes de informação de Fisher deste modelo. 


\subsubsection{Funções escore e informação de Fisher}

As funções escore penalizadas são definidas como as derivadas do logaritmo da função de verossimilhança penalizada em relação a cada parâmetro. Estas funções, para este modelo, são definidas da seguinte forma:

$$
\begin{aligned}
& \mathbf{U}_{\mathrm{p}}(\boldsymbol{\beta})=\frac{\partial \mathbf{L}_{\mathrm{p}}(\boldsymbol{\theta}, \alpha)}{\partial \boldsymbol{\beta}}=\frac{1}{\phi} \mathbf{X}^{\top} \mathbf{D}(\mathbf{v}) \epsilon, \\
& \mathbf{U}_{\mathrm{p}}(\mathbf{f})=\frac{\partial \mathbf{L}_{\mathrm{p}}(\boldsymbol{\theta}, \alpha)}{\partial \mathbf{f}}=\frac{1}{\phi} \mathbf{N}^{\top} \mathbf{D}(\mathbf{v}) \epsilon-\alpha \mathbf{K} \mathbf{f} \\
& U_{\mathrm{p}}(\phi)=\frac{\partial L_{\mathrm{p}}(\boldsymbol{\theta}, \alpha)}{\partial \phi}=(2 \phi)^{-1}\left\{\phi^{-1} \boldsymbol{\epsilon}^{\top} \mathbf{D}(v) \boldsymbol{\epsilon}-n\right\},
\end{aligned}
$$

em que $\mathbf{D}(\mathbf{v})=\operatorname{diag}\left\{v_{1}, \ldots, v_{n}\right\} \operatorname{com} v_{i}=-2 W_{g}\left(u_{i}\right)$. A função $W_{g}$ é definida por

$$
W_{g}(u)=\frac{g^{\prime}(u)}{g(u)} .
$$

Na Tabela 2.1, extraída de Cysneiros [2004], temos as expressões para $W_{g}(u)$ para algums distribuições pertencentes à classe simétrica.

Por meio das funções escores, também é possível obter a matriz Hessiana. As submatrizes da matriz Hessiana ficam dadas por

$$
\begin{aligned}
& \mathbf{L}_{\beta \beta}=\frac{\partial^{2} L_{\mathrm{p}}(\boldsymbol{\theta}, \alpha)}{\partial \boldsymbol{\beta} \partial \boldsymbol{\beta}^{\top}}=-\frac{1}{\phi} \mathbf{X}^{\top} \mathbf{D}(\mathbf{a}) \mathbf{X}, \\
& \mathbf{L}_{f f}=\frac{\partial^{2} L_{\mathrm{p}}(\boldsymbol{\theta}, \alpha)}{\partial \mathbf{f} \partial \mathbf{f}^{\top}}=-\frac{1}{\phi} \mathbf{N}^{\top} \mathbf{D}(\mathbf{a}) \mathbf{N}-\alpha \mathbf{K}, \\
& L_{\phi \phi}=\frac{\partial^{2} L_{\mathrm{p}}(\boldsymbol{\theta}, \alpha)}{\partial \phi^{2}}=\frac{1}{\phi^{2}}\left\{\frac{n}{2}+\mathbf{u}^{\top} \mathbf{D}(\mathbf{c}) \mathbf{u}-\frac{1}{\phi} \boldsymbol{\epsilon}^{\top} \mathbf{D}(\mathbf{v}) \boldsymbol{\epsilon}\right\}, \\
& \mathbf{L}_{\beta f}=\frac{\partial^{2} L_{\mathrm{p}}(\boldsymbol{\theta}, \alpha)}{\partial \boldsymbol{\beta} \partial \mathbf{f}^{\top}}=-\frac{1}{\phi} \mathbf{X}^{\top} \mathbf{D}(\mathbf{a}) \mathbf{N}, \\
& \mathbf{L}_{\beta \phi}=\frac{\partial^{2} L_{\mathrm{p}}(\boldsymbol{\theta}, \alpha)}{\partial \boldsymbol{\beta} \partial \phi}=\frac{2}{\phi^{2}} \mathbf{X}^{\top} \mathbf{b} \quad \mathrm{e} \\
& \mathbf{L}_{f \phi}=\frac{\partial^{2} L_{\mathrm{p}}(\boldsymbol{\theta}, \alpha)}{\partial \mathbf{f} \partial \phi}=\frac{2}{\phi^{2}} \mathbf{N}^{\top} \mathbf{b},
\end{aligned}
$$


em que, $\mathbf{D}(\mathbf{a})=\operatorname{diag}\left\{a_{1}, \ldots, a_{n}\right\}$ e $\mathbf{D}(\mathbf{c})=\operatorname{diag}\left\{c_{1}, \ldots, c_{n}\right\}$. Sendo $a_{i}=$ $-2\left\{W_{g}\left(u_{i}\right)+2 u_{i} W_{g}^{\prime}\left(u_{i}\right)\right\}, c_{i}=W_{g}^{\prime}\left(u_{i}\right)$ e $\mathbf{b}=\left(b_{1}, \ldots, b_{n}\right)^{\top}, \boldsymbol{\epsilon}=\left(\epsilon_{1}, \ldots, \epsilon_{n}\right)^{\top} \mathrm{e}$ $\mathbf{u}=\left(u_{1}, \ldots, u_{n}\right)^{\top}, \operatorname{com} b_{i}=\left\{W_{g}\left(u_{i}\right)+u_{i} W_{g}^{\prime}\left(u_{i}\right)\right\} \epsilon_{i}$ e $\epsilon_{i}=y_{i}-\mu_{i}$.

Tabela 2.1: Expressões para $W_{g}(u)$ e $W_{g}^{\prime}(u)$ para algumas distribuições simétricas.

\begin{tabular}{|c||c|c|}
\hline Distribuição & $W_{g}(u)$ & $W_{g}^{\prime}(u)$ \\
\hline \hline Normal & $-\frac{1}{2}$ & 0 \\
t-Student & $-\frac{v+1}{2(v+u)}$ & $\frac{v+1}{2(v+u)^{2}}$ \\
t-Student generalizada & $-\frac{(r+1)}{2(s+u)}$ & $\frac{(r+1)}{2(s+u)^{2}}$ \\
Logística I & $-\tanh \left(\frac{u}{2}\right)$ & $-\frac{\operatorname{sech}\left(\frac{u}{2}\right)}{2}$ \\
Logística-II & $-\frac{\exp (-\sqrt{u})-1}{(-2 \sqrt{u})[1+\exp (-\sqrt{u})]}$ & $\frac{2 \exp (-\sqrt{u}) \sqrt{u}+\exp (-2 \sqrt{u})-1}{-4 u^{3 / 2}[1+\exp (-\sqrt{u})]^{2}}$ \\
Logística generalizada & $\frac{-\alpha m[\exp (-\alpha \sqrt{u})-1]}{(-2 \sqrt{u})[1+\exp (-\alpha \sqrt{u})]}$ & $-\frac{\alpha m}{4} \frac{2 \alpha \exp (-\alpha \sqrt{u}) \sqrt{u}+\exp (-2 \alpha \sqrt{u})-1}{u^{3 / 2}[1+\exp (-\alpha \sqrt{u})]^{2}}$ \\
Exponencial potência & $-\frac{1}{2(1+k) u^{k /(k+1)}}$ & $\frac{k}{(1+k)^{2} 2 u^{(2 k+1) /(1+k)}}$ \\
\hline \hline
\end{tabular}

Agora, com as matrizes obtidas acima, é possível obter a matriz de informação de Fisher, que é definida como $\mathbf{K}_{\theta \theta}=-E\left[\ddot{\mathbf{L}}_{\theta \theta}\right]$. Para o modelo em questão, pode-se mostrar que as submatrizes da matriz de informação de Fisher são dados por

$$
\begin{gathered}
\mathbf{K}_{\beta \beta}=\mathrm{E}\left\{-\frac{\partial^{2} L_{\mathrm{p}}(\boldsymbol{\theta}, \alpha)}{\partial \boldsymbol{\beta} \partial \boldsymbol{\beta}^{\top}}\right\}=\frac{4 d_{g}}{\phi} \mathbf{X}^{\top} \mathbf{X} \\
\mathbf{K}_{f f}=\mathrm{E}\left\{-\frac{\partial^{2} L_{\mathrm{p}}(\boldsymbol{\theta}, \alpha)}{\left.\partial \mathbf{f} \partial \mathbf{f}^{\top}\right\}}=\left\{\frac{4 d_{g}}{\phi} \mathbf{N}^{\top} \mathbf{N}+\alpha \mathbf{K}\right\}\right. \\
\mathbf{K}_{\beta f}=\mathrm{E}\left\{-\frac{\partial^{2} L_{\mathrm{p}}(\boldsymbol{\theta}, \alpha)}{\left.\partial \boldsymbol{\beta} \partial \mathbf{f}^{\top}\right\}}=\frac{4 d_{g}}{\phi} \mathbf{X}^{\top} \mathbf{N}\right. \\
K_{\phi \phi}=\mathrm{E}\left\{-\frac{\partial^{2} L_{\mathrm{p}}(\boldsymbol{\theta}, \alpha)}{\partial \phi^{2}}\right\}=\frac{n}{4 \phi^{2}}\left(4 f_{g}-1\right), \\
\mathbf{K}_{\beta \phi}=\mathrm{E}\left\{-\frac{\partial^{2} L_{\mathrm{p}}(\boldsymbol{\theta}, \alpha)}{\partial \boldsymbol{\beta} \partial \phi}\right\}=\mathbf{0}_{(p \times 1)} \mathrm{e} \quad \mathbf{K}_{f \phi}=\mathrm{E}\left\{-\frac{\partial^{2} L_{\mathrm{p}}(\boldsymbol{\theta}, \alpha)}{\partial \mathbf{f} \partial \phi}\right\}=\mathbf{0}_{(k \times 1)},
\end{gathered}
$$


com $f_{g}=E\left[W_{g}^{2}\left(U^{2}\right) U^{4}\right]$ e $d_{g}=E\left[W_{g}^{2}\left(U^{2}\right) U^{2}\right]$, sendo $\mathrm{U} \sim \mathrm{S}(0,1)$. A Tabela 2.2, extraída de Cysneiros [2004], apresenta os valores de $d_{g}$ e $f_{g}$ para algumas distribuições simétricas. Além desses valores, a tabela também traz o valor de $\xi$, relacionado com a variância de cada distribuição.

Tabela 2.2: Valores de $d_{g}, f_{g}$ e $\xi$ para algumas distribuições simétricas.

\begin{tabular}{|c||c|c|c|}
\hline Distribuição & $d_{g}$ & $f_{g}$ & $\xi$ \\
\hline \hline Normal & $\frac{1}{4}$ & $\frac{3}{4}$ & 1 \\
t-Student & $\frac{v+1}{4(v+3)}$ & $\frac{3(v+1)}{4(v+3)}$ & $\frac{v}{v-2}, v>2$ \\
t-Student generalizada & $\frac{r(r+1)}{4 s(r+3)}$ & $\frac{3(r+1)}{4(r+3)}$ & $\frac{s}{r-2}, s>0, r>2$ \\
Logística I & 0,369310044 & 1,003445984 & 0,79569 \\
Logística-II & $\frac{1}{12}$ & 0,60749 & $\pi^{2} / 3$ \\
Logística generalizada & $\frac{\alpha^{2} m^{2}}{4(2 m+1)}$ & $\frac{2 m\left(2+m^{2} \psi^{\prime}(m)\right)}{4(2 m+1)}$ & $2 \psi^{\prime}(m)$ \\
Exponencial potência & $\frac{\Gamma\{(3-k) / 2\}}{4\left(2^{k-1}\right)(1+k)^{2} \Gamma\{(k+1) / 2\}}$ & $\frac{(k+3)}{4(k+1)}$ & $2^{(1+k)} \frac{\Gamma\{3(k+1) / 2\}}{\Gamma\{(k+1) / 2\}}$ \\
\hline \hline
\end{tabular}

\subsubsection{Procedimentos de estimação}

Ibacache-Pulgar et al. [2012] propõem alguns procedimentos para a estimação dos parâmetros do modelo definido nesta seção que combina procedimentos do método escore de Fisher e do algoritmo backfitting. O objetivo de tais métodos é maximizar o logaritmo da função de verossimilhança penalizada. Não apresentaremos tais procedimentos nesta seção, no entanto, o mesmo procedimento será utilizado para a estimação dos parâmetros do modelo desenvolvido neste trabalho, logo uma explicação mais detalhada do método será apresentada nos capítulos seguintes.

Por meio do método de backfitting é possível obter, generalizando com resultados conhecidos dos modelos de regressão lineares, uma fórmula para o cálculo do número de graus de liberdade efetivos. Esta fórmula, que é função do parâmetro de suavização, fica dada por

$$
d f(\alpha)=\operatorname{traço}\left\{\left(\mathbf{N}^{\top} \mathbf{D}(\mathbf{v}) \mathbf{N}+\phi \alpha \mathbf{K}\right)^{-1}\left(\mathbf{N}^{\top} \mathbf{D}(\mathbf{v}) \mathbf{N}\right)\right\}
$$




\subsection{Modelos com erros autoregressivos}

Após apresentarmos os modelos não paramétricos e os modelos semiparamétricos, apresentamos nesta seção os modelos de regressão linear com erros autoregressivos. Há diversas literaturas especializadas, uma delas é Neter et al. [1996], em que nos baseamos para introduzir este assunto.

$\mathrm{Na}$ prática, há diversas situações em que pode existir autocorrelação dos erros, em que o erro de hoje depende do erro de ontem, principalmente em unidades de observação obtidas ao longo do tempo. Caso realmente exista a presença deste fenômeno nos erros e nosso modelo não contemple tal fato, estaremos estimando os parâmetros do modelo de forma não tão satisfatória quanto seria possível, enviesando as estimativas de variância.

Dentre os problemas que ocorrem ao não incluirmos autocorrelação nos erros quando esta existe, podemos destacar:

- Apesar de continuarem sendo não viesados, os parâmetros estimados não serão mais os estimadores não viesados de variância uniformemente mínima.

- O erro quadrático médio do modelo será subestimado. Portanto, o erro padrão das estimativas dos parâmetros também serão subestimados.

- Como consequência deste último ponto, os intervalos de confiança e os testes de hipótese para os parâmetros do modelo não poderão ser mais utilizados.

Portanto, dos pontos acima, podemos perceber como é importante incluir no modelo a presença desta estrutura de correlação a fim de evitarmos tais problemas.

Assim, o modelo de regressão linear com erros autorregressivos de ordem 1 é definido através das duas equações

$$
\begin{gathered}
y_{t}=\beta_{0}+\beta_{1} x_{t}+\epsilon_{t}, \\
\epsilon_{t}=\rho \epsilon_{t-1}+u_{t} .
\end{gathered}
$$

No modelo acima, consideramos que $u_{t}$ são variáveis aleatórias independentes e identicamente distribuídas de acordo com uma normal de média 0 e variância igual a $\sigma^{2}$. Vale ressaltar que o parâmetro $\rho$ do modelo acima é condicionado a ser menor do que 1 em valor absoluto. 
Podemos expandir o modelo acima considerando um número maior de variáveis explicativas de forma similar.

Este modelo de regressão apresenta algumas propriedades interessantes. Dentre elas, podemos destacar que a correlação imposta aos erros é função do parâmetro $\rho$. Mais precisamente, temos que a correlação entre erros defasados em $q$ unidades é igual a $\rho^{q}$.

Geralmente, o pesquisador verifica a presença de erros autoregressivos através do gráfico dos resíduos ao longo do tempo. Caso esse gráfico não nos mostre pontos aleatórios em torno do 0, os erros podem ser autocorrelacionados. Há, ainda, um teste estatístico para detectar tal fenômeno, o teste de DurbinWatson. As hipóteses do teste são:

$$
\begin{aligned}
& H_{0}: \rho=0, \\
& H_{1}: \rho>0 .
\end{aligned}
$$

O motivo da hipótese alternativa ser maior do que 0 é que, na maioria dos trabalhos os erros são positivamente correlacionados.

A estatística do teste é função dos resíduos $\hat{e}_{t}$, isto é, dos valores obervados menos os valores previstos. Podemos escrevê-la como:

$$
D=\frac{\sum_{t=2}^{n}\left(\hat{e}_{t}-\hat{e}_{t-1}\right)^{2}}{\sum_{t=1}^{n} \hat{e}_{t}^{2}} .
$$

Durbin e Watson tabularam alguns valores críticos do teste que dependem de $n$ e da significância desejada. Mais precisamente, estão tabulados os limites inferior e superior, que chamaremos de $d_{1}$ e $d_{2}$ respectivamente. Valores baixos de $D$ tendem a rejeitar $H_{0}$. Isso ocorre porque na presença de autocorrelação, resíduos sequentes tendem a ser da mesma grandeza, o que faz com que a estatística $D$ se torne pequena.

Temos as seguintes decisões:

- Se $D>d_{2}$, não rejeitamos $H_{0}$.

- Se $D<d_{1}$, rejeitamos $H_{0}$.

- Caso contrário, o resultado do teste será inconclusivo.

Na literatura de modelos lineares com erros autoregressivos há diversos procedimentos de estimação. Neste trabalho, iremos apresentar o método de Cochrane-Orcutt. O descreveremos utilizando o modelo de regressão linear simples. Analogamente, pode ser estendido para o caso de regressão múltipla. 
O primeiro passo deste método é ajustar um modelo de regressão linear supondo erros independentes. Com este modelo, podemos encontrar os resíduos $\hat{e}_{t}$. O próximo passo do método é ajustar o seguinte modelo:

$$
\hat{e}_{t}=\rho \hat{e}_{t-1}+u_{t},
$$

em que $u_{t}$ são erros independentes. Podemos fazê-lo isso facilmente pelo método de mínimos quadrados. Teremos, portanto, a estimativa de $\rho$, que chamaremos de $\hat{\rho}$. Com este valor, obtemos

$$
\begin{gathered}
\tilde{y}_{t}=y_{t}-\hat{\rho} y_{t-1} \quad \mathrm{e} \\
\tilde{x}_{t}=x_{t}-\hat{\rho} x_{t-1} .
\end{gathered}
$$

Podemos agora ajustar o seguinte modelo

$$
\tilde{y}_{t}=\tilde{\beta}_{0}+\tilde{\beta}_{1} \tilde{x}_{t}+u_{t}
$$

em que mais uma vez, $u_{t}$ são erros independentes. Temos uma relação entre os parâmetros $\tilde{\beta}_{0}, \tilde{\beta}_{1}$ com os parâmetros originais $\beta_{0}$ e $\beta_{1}$ dada por

$$
\begin{gathered}
\beta_{0}=\frac{\tilde{\beta}_{0}}{1-\hat{\rho}} \mathrm{e} \\
\beta_{1}=\tilde{\beta}_{1} .
\end{gathered}
$$

Com esta relação, também encontramos as variâncias das estimativas dos parâmetros

$$
\begin{gathered}
\operatorname{Var}\left(\hat{\beta}_{0}\right)=\frac{\operatorname{Var}\left(\hat{\tilde{\beta}}_{0}\right)}{(1-\hat{\rho})^{2}} \quad \mathrm{e} \\
\operatorname{Var}\left(\hat{\beta}_{1}\right)=\operatorname{Var}\left(\hat{\tilde{\beta}}_{1}\right) .
\end{gathered}
$$

Com o ajuste deste modelo com as variáveis transformadas, podemos realizar o teste de Durbin-Watson para verificar se os resíduos desta nova estimação ainda possuem autocorrelação. Se rejeitarmos que ainda há a presença de autocorrelação, o procedimento termina. Podemos encontrar os valores originais do modelo e suas variâncias por meio das relações acima.

Caso ainda tenha a presença de autocorrelação nos erros, encontraremos os parâmetros nas formas originais, através das relações acima e com estes novos parâmetros calculamos novos resíduos, que serão utilizados para reestimar o 
parâmetro $\rho$. Assim, repetimos o processo até que o teste de Durbin-Watson não rejeite a hipótese de resíduos não autocorrelacionaodos. Outro possível critério de parada é definir um limite para a variação do parâmetro $\rho$.

Em geral, o procedimento realiza poucas iterações.

Um problema no procedimento de Cochrane-Orcutt descrito acima é a perda da primeira observação, visto que não conseguimos realizar a transformação necessária. Para contornar tal problema, surgiu o método de PraisWinsten, que utiliza as mesmas transformações do método de Orcutt para $t$ maior do que 2 e quando $t$ é igual a 1 , utiliza o seguinte:

$$
\begin{gathered}
\tilde{y_{1}}=\left(\sqrt{1-\rho^{2}}\right) y_{1} \quad \mathrm{e} \\
\tilde{x}_{1}=\left(\sqrt{1-\rho^{2}}\right) x_{1} .
\end{gathered}
$$

Os próximos passos desse método são idênticos aos passos do procedimento de Cochrane-Orcutt.

\subsection{Conclusões do capítulo}

Neste capítulo, apenas introduzimos algumas classes de modelos para uma melhor compreensão dos capítulos seguintes. No presente trabalho, apresentamos algumas propriedades dos modelos parcialmente lineares com erros simétricos autoregressivos de primeira ordem. Os modelos que serão apresentados nos capítulos posteriores possuem componentes paramétricos e componentes não paramétricos assim como os modelos semiparamétricos. A diferença está nas suposições dos erros, em que no modelo aqui introduzido, eles são provenientes de uma distribuição simétrica e apresentam autocorrelação. Mais precisamente, o modelo supõe que as observações são correlacionadas de acordo com um processo AR(1). Tratam-se de generalizações das metodologias que foram brevemente discutidas neste capítulo. 


\section{Capítulo 3}

\section{Modelos parcialmente lineares simétricos $\mathrm{AR}(1)$}

Neste capítulo, introduzimos os modelos parcialmente lineares simétricos AR(1). Na Seção 3.1, apresentamos a classe e derivamos alguns resultados básicos. Já na Seção 3.2, derivamos as funções escore para posteriormente estimarmos os parâmetros de interesse. Nesta seção, também apresentamos a notação matricial utilizada neste trabalho. Na Seção 3.3, obtemos a matriz de Fisher observada e a matriz de informação de Fisher. Enquanto que na Seção 3.4, discutimos formas de estimar os parâmetros do modelo proposto. Por fim, na Seção 3.5, apresentamos alguns resultados assintóticos.

\subsection{Descrição do modelo}

O modelo parcialmente linear com erros simétricos autoregressivos de primeira ordem é definido da seguinte forma:

$$
\begin{gathered}
y_{i}=\mathbf{x}_{i}^{\top} \boldsymbol{\beta}+f\left(t_{i}\right)+\epsilon_{i}, \\
\epsilon_{i}=\rho \epsilon_{i-1}+e_{i}, \quad-1<\rho<1,
\end{gathered}
$$

em que $e_{i}, i=1, \ldots, n$, são variáveis aleatórias independentes que seguem uma distribuição simétrica de média 0 e parâmetro de dispersão igual a $\phi$. Para utilizarmos todas as observações, tomemos que $\epsilon_{0}=0$, fato comumente utilizado (vide Neter et al. [1996]). Na notação usual, $e_{i} \sim \mathrm{S}(0, \phi)$. A função $f\left(t_{i}\right)$ representa uma função suave desconhecida que depende da covariável $t$ que contribui de maneira não paramétrica sob a variável resposta. Vamos 
denotar aqui como $\boldsymbol{\eta}_{i}^{\top} \mathbf{f}$. Temos ainda que os vetores $\boldsymbol{\beta}, \mathbf{f}$ são parâmetros a serem estimados no modelo, assim como $\phi$ e $\rho$.

Assim como no Capítulo 2, o vetor f é igual a $\left(f\left(t_{1}^{0}\right), \ldots, f\left(t_{q}^{0}\right)\right)^{\top}$, em que $q$ representa o número de pontos distintos da variável $t$. Assim $\mathbf{f}$ é um vetor de dimensão $q \times 1$. O vetor $\boldsymbol{\eta}_{i}$ representa a $i$-ésima linha da matriz $\mathbf{N}$, definida da mesma forma que anteriormente.

Alternativamente, podemos escrever o modelo da seguinte maneira:

(i) $y_{1} \sim S\left(\mu_{1}, \phi\right)$ e $y_{i} \mid y_{i-1}, \ldots, y_{1}$ independentes $S\left(\mu_{i}, \phi\right)(i=2, \ldots, n)$,

(ii) $\quad \mu_{1}=\boldsymbol{x}_{1}^{\top} \boldsymbol{\beta}+f\left(t_{1}\right)$ e $\mu_{i}=\boldsymbol{x}_{i}^{\top} \boldsymbol{\beta}+f\left(t_{i}\right)+\rho\left(y_{i-1}-\boldsymbol{x}_{i-1}^{\top} \boldsymbol{\beta}-f\left(t_{i-1}\right)\right)$

$(i=2, \ldots, n)$.

Mesmo podendo ser facilmente estendido para mais funções não paramétricas, apresentamos os cálculos deste capítulo supondo apenas uma função com o objetivo de facilitar o entendimento.

Outro ponto a se destacar, é que consideramos apenas as distribuições simétricas que satisfazem as condições de regularidade.

Na próxima Subseção, 3.1.1, derivamos os dois primeiros momentos do modelo descrito neste capítulo e apresentamos a covariância entre diferentes observações.

\subsubsection{Derivação dos dois primeiros momentos}

Os resultados apresentados nesta seção são derivados com maiores detalhes no Apêndice A, onde obtemos que

$$
\begin{gathered}
E\left[y_{k}\right]=\mathbf{x}_{k}^{\top} \boldsymbol{\beta}+\boldsymbol{\eta}_{k}^{\top} \mathbf{f}, \quad \forall k, \\
\operatorname{Var}\left[y_{k}\right]=\phi \xi \sum_{j=0}^{k-1} \rho^{2 j} .
\end{gathered}
$$

Além de $E\left[y_{k}\right]$ e $\operatorname{Var}\left[y_{k}\right]$, também podemos derivar as covariâncias entre duas observações. Através dos cálculos presentes no Apêndice A, é possível encontrar a covariância entre diferentes observações.

$$
\text { Para }|j-k|>1
$$




$$
\begin{array}{r}
\operatorname{Cov}\left(y_{k}, y_{j}\right)=\rho^{2} \operatorname{Cov}\left(y_{k-1}, y_{j-1}\right)=\rho^{4} \operatorname{Cov}\left(y_{k-2}, y_{j-2}\right)=\cdots= \\
=\rho^{2[\min (k, j)-1]} \operatorname{Cov}\left(y_{1}, y_{[|j-k|+1]}\right) .
\end{array}
$$

Já, para $|j-k|=1$,

$$
\begin{array}{r}
\operatorname{Cov}\left(y_{k}, y_{k+1}\right)=\rho^{2} \operatorname{Cov}\left(y_{k-1}, y_{k}\right)+\rho \xi \phi= \\
=\rho^{4} \operatorname{Cov}\left(y_{k-2}, y_{k-1}\right)+\rho^{3} \xi \phi+\rho \xi \phi=\rho^{2[k-1]} \operatorname{Cov}\left(y_{1}, y_{2}\right)+\sum_{i=0}^{k-2} \rho^{(2 i+1)} \xi \phi
\end{array}
$$

em que

$$
\operatorname{Cov}\left(y_{1}, y_{2}\right)=\rho \phi \xi \quad \text { e } \operatorname{Cov}\left(y_{k}, y_{k+1}\right)=\sum_{i=0}^{k-1} \rho^{(2 i+1)} \xi \phi .
$$

Através das expressões (3.3) e (3.5), notamos claramente que para $k$ grande apenas as observações consecutivas apresentam correlação.

Com os cálculos derivados nesta seção e com as informações apresentadas no Capítulo 2, podemos notar em quais situações o modelo proposto pode ser recomendado. Listamos as seguintes situações:

- Quando não é conhecida a relação entre uma ou mais variáveis preditoras e a variável resposta, não existindo um alto interesse em estudar os seus efeitos, apenas controlá-los. Essas variáveis, portanto, podem ser consideradas de maneira não paramétrica.

- Quando as observações são correlacionadas, principalmente as observações consecutivas.

- Quando há indícios de que a distribuição da variável resposta tem caudas mais pesadas ou mais leves do que a esperada sob normalidade

Como exemplo prático das situações acima, podemos ter uma variável resposta medida ao longo do tempo, o que pode implicar em uma correlação serial. Em várias situações, o interesse não é estudar a variável ao longo do tempo e sim estudar a relação com outras variáveis, sugerindo controlar o tempo por meio de um efeito não paramétrico. Todavia, pode haver indícios após o ajuste 
de modelos parcialmente lineares normais com estrutura $\mathrm{AR}(1)$ de erros com caudas mais pesadas do que a esperada sob normalidade, sugerindo a suposição de distribuição para os erros com curtose maior do que sob a distribuição normal, que apresenta curtose igual a 3, como por exemplo a distribuição t de Student.

\subsection{Funções escore}

Para o cálculo das funções escore, temos que encontrar primeiramente a função de verossimilhança do modelo. Podemos reescrever a função de distribuição conjunta por meio da seguinte relação:

$f_{Y}\left(y_{1}, \ldots, y_{n}\right)=f_{y_{n}}\left(y_{n} \mid y_{n-1}, \ldots, y_{1}\right) f_{y_{n-1}}\left(y_{n-1} \mid y_{n-2}, \ldots, y_{1}\right) \ldots f_{y_{2}}\left(y_{2} \mid y_{1}\right) f_{y_{1}}\left(y_{1}\right)$

Similarmente aos modelos lineares simétricos com estrutura AR(1) (vide Cao et al. [2010]), vamos supor que $y_{i} \mid y_{i-1} \sim \mathrm{S}\left(\mu_{i}, \phi\right)$, com $\mu_{i}=\mathbf{x}_{i}^{\top} \boldsymbol{\beta}+\boldsymbol{\eta}_{i}^{\top} \mathbf{f}+$ $\rho\left(y_{i-1}-\mathbf{x}_{i-1}^{\top} \boldsymbol{\beta}-\boldsymbol{\eta}_{i-1}^{\top} \mathbf{f}\right)$. Logo, em anologia aos modelos lineares simétricos apresentados no segundo capítulo, temos que o logaritmo da função de verossimilhança, utilizando a notação comumente utilizada em modelos simétricos, pode ser escrita na seguinte forma:

$$
L(\boldsymbol{\theta})=-\frac{n}{2} \log \phi+\sum_{i=1}^{n} \log \left\{g\left(u_{i}\right)\right\},
$$

em que,

$$
u_{i}=\frac{\left(\epsilon_{i}-\rho \epsilon_{i-1}\right)^{2}}{\phi}, \epsilon_{i}=y_{i}-\mathbf{x}_{i}^{\top} \boldsymbol{\beta}-\boldsymbol{\eta}_{i}^{\top} \mathbf{f} \quad e \quad \epsilon_{0}=0
$$

A função $g($.$) , conforme explicado anteriormente, depende da distribuição$ simétrica que está sendo utilizada.

De maneira similar aos modelos parcialmente lineares com erros simétricos, utilizaremos a função de verossimilhança penalizada, em que o logaritmo da função de verossimilhança penalizada pode ser expresso por:

$$
L_{\mathrm{p}}(\boldsymbol{\theta})=-\frac{n}{2} \log \phi+\sum_{i=1}^{n} \log \left\{g\left(u_{i}\right)\right\}-\frac{\alpha}{2} \mathbf{f}^{\top} \mathbf{K f} .
$$

Como já destacamos, $\alpha$ representa o parâmetro de suavização e pode va- 
riar entre 0 e $\infty$. A escolha do valor de $\alpha$ é muito importante, visto que $\alpha$ controla a relação entre um super ajustamento e a suavização da função. $O$ método penalizado é conveniente para evitar um super ajustamento sem a necessidade de uma escolha adequada do número de nós. Já a matriz $\mathbf{K}$ é uma matriz $q \times q$ definida da mesma forma do que no capítulo anterior. $\mathrm{O}$ vetor $\mathbf{f}=\left(f_{1}, \ldots, f_{q}\right)^{\top}$ representa os parâmetros associados às variáveis explicativas utilizadas de maneira não paramétrica.

Após encontrarmos o logaritmo da função de verossimilhança penalizada, podemos prosseguir para o cálculo das funções escores em relação ao parâmetro $\boldsymbol{\theta}$ que são dadas por

$$
\begin{aligned}
\mathbf{U}_{\theta} & =\left(\mathbf{U}_{\beta}^{\top}, \mathbf{U}_{f}^{\top}, U_{\phi}, U_{\rho}\right)^{\top}, \quad \text { em que } \\
\mathbf{U}_{\theta} & =\frac{\partial L_{\mathrm{p}}(\boldsymbol{\theta})}{\partial \boldsymbol{\theta}}, \quad \mathbf{U}_{\beta}=\frac{\partial L_{\mathrm{p}}(\boldsymbol{\theta})}{\partial \boldsymbol{\beta}} \\
\mathbf{U}_{f} & =\frac{\partial L_{\mathrm{p}}(\boldsymbol{\theta})}{\partial \mathbf{f}}, \quad U_{\phi}=\frac{\partial L_{\mathrm{p}}(\boldsymbol{\theta})}{\partial \phi} \\
U_{\rho} & =\frac{\partial L_{\mathrm{p}}(\boldsymbol{\theta})}{\partial \rho} .
\end{aligned}
$$

Nesta seção apresentamos os resultados dessas funções, no entanto, os cálculos com maiores detalhes podem ser vistos no Apêndice B.

Antes de apresentarmos as fórmulas matriciais dessa função, definiremos algumas matrizes que são comumente utilizadas em modelos simétricos. As matrizes A e B definidas abaixo foram usadas em Cao et al. [2010],

$$
\begin{aligned}
& \mathbf{A}=\left(\begin{array}{rrrrr}
1 & 0 & 0 & \ldots & 0 \\
-\rho & 1 & 0 & \ldots & 0 \\
\vdots & & \vdots & & \vdots \\
0 & \ldots & 0 & -\rho & 1
\end{array}\right), \\
& \mathbf{B}=\left(\begin{array}{rrrrr}
0 & 0 & 0 & \ldots & 0 \\
-1 & 0 & 0 & \ldots & 0 \\
0 & -1 & 0 & \ldots & 0 \\
\vdots & & \vdots & & \\
0 & \ldots & 0 & -1 & 0
\end{array}\right),
\end{aligned}
$$


$\mathbf{D}(\mathbf{v})=\operatorname{diag}\left(v_{1}, \ldots, v_{n}\right), \operatorname{com} v_{i}=-2 W_{g}\left(u_{i}\right)$ e $W_{g}(u)=\frac{g^{\prime}(u)}{g(u)}$,

$\mathbf{D}(\mathbf{m})=\operatorname{diag}\left(m_{1}, \ldots, m_{n}\right), m_{i}=v_{i} u_{i}$,

$\mathbf{1}_{n}=(1, \ldots, 1)^{\top}$

$\boldsymbol{\epsilon}=\left(\epsilon_{1}, \ldots, \epsilon_{n}\right)^{\top}$

$\mathbf{X}$ é a matriz contendo os valores das variáveis explicativas utilizadas de forma paramétrica de todas observações e

$\mathbf{N}$ é uma matriz $q \times q$ chamada de matriz de incidência, formada por valores 0 e 1, definida da mesma forma que no capítulo anterior.

Com a criação dessas matrizes, podemos escrever as funções escore da seguinte forma:

$$
\begin{aligned}
\mathbf{U}_{\beta} & =\frac{\partial L_{\mathrm{p}}(\boldsymbol{\theta})}{\partial \boldsymbol{\beta}}=(\mathbf{A X})^{\top} \mathbf{D}(\mathbf{v}) \phi^{-1} \mathbf{A} \boldsymbol{\epsilon}, \\
\mathbf{U}_{f} & =\frac{\partial L_{\mathrm{p}}(\boldsymbol{\theta})}{\partial \mathbf{f}}=(\mathbf{A N})^{\top} \mathbf{D}(\mathbf{v}) \phi^{-1} \mathbf{A} \boldsymbol{\epsilon}-\alpha \mathbf{K f}, \\
U_{\phi} & =\frac{\partial L_{\mathrm{p}}(\boldsymbol{\theta})}{\partial \phi}=\frac{1}{2 \phi} \mathbf{1}_{n}^{\top}\left(\mathbf{D}(\mathbf{m}) \mathbf{1}_{n}-\mathbf{1}_{n}\right) \quad \mathrm{e} \\
U_{\rho} & =\frac{\partial L_{\mathrm{p}}(\boldsymbol{\theta})}{\partial \rho}=-\phi^{-1} \boldsymbol{\epsilon}^{\top} \mathbf{B}^{\top} \mathbf{D}(\mathbf{v}) \mathbf{A} \boldsymbol{\epsilon} .
\end{aligned}
$$

A partir dessas matrizes, a manipulação computacional dessas funções se torna muito mais atrativa, facilitando a estimação dos parâmetros do modelo.

\subsection{Informação de Fisher}

Nesta seção, apresentamos a matriz de informação de Fisher, obtendo primeiramente a matriz de Fisher observada, que é definida por

$$
-\ddot{\mathbf{L}}_{\theta \theta}=\frac{\partial^{2} L_{\mathrm{p}}(\boldsymbol{\theta})}{\partial \boldsymbol{\theta} \partial \boldsymbol{\theta}^{\top}}
$$

em que $\ddot{\mathbf{L}}_{\theta \theta}$ é a matriz Hessiana dada por 


$$
\ddot{\mathbf{L}}_{\theta \theta}=\left(\begin{array}{cccc}
\ddot{\mathbf{L}}_{\beta \beta} & \ddot{\mathbf{L}}_{\beta f} & \ddot{\mathbf{L}}_{\beta \phi} & \ddot{\mathbf{L}}_{\beta \rho} \\
\ddot{\mathbf{L}}_{f \beta} & \ddot{\mathbf{L}}_{f f} & \ddot{\mathbf{L}}_{f \phi} & \ddot{\mathbf{L}}_{f \rho} \\
\ddot{\mathbf{L}}_{\phi \beta} & \ddot{\mathbf{L}}_{\phi f} & \ddot{\mathbf{L}}_{\phi \phi} & \ddot{\mathbf{L}}_{\phi \rho} \\
\ddot{\mathbf{L}}_{\rho \beta} & \ddot{\mathbf{L}}_{\rho f} & \ddot{L}_{\rho \phi} & \ddot{L}_{\rho \rho}
\end{array}\right)
$$

$\operatorname{com} \ddot{\mathbf{L}}_{\beta \beta}, \ddot{\mathbf{L}}_{f f}, \ddot{L}_{\phi \phi}, \ddot{L}_{\rho \rho}, \ddot{\mathbf{L}}_{\beta f}, \ddot{\mathbf{L}}_{\beta \phi}, \ddot{\mathbf{L}}_{\beta \rho}, \ddot{\mathbf{L}}_{f \phi}, \ddot{\mathbf{L}}_{f \rho}, \ddot{L}_{\phi \rho}$ sendo

$$
\begin{aligned}
& \ddot{\mathbf{L}}_{f \beta}=\ddot{\mathbf{L}}_{\beta f}^{\top}, \ddot{\mathbf{L}}_{\phi \beta}=\ddot{\mathbf{L}}_{\beta \phi}^{\top}, \\
& \ddot{\mathbf{L}}_{\rho \beta}=\ddot{\mathbf{L}}_{\beta \rho}^{\top}, \ddot{\mathbf{L}}_{f \phi}=\ddot{\mathbf{L}}_{\phi f}^{\top}, \\
& \ddot{\mathbf{L}}_{f \rho}=\ddot{\mathbf{L}}_{\rho f}^{\top}, \ddot{\mathbf{L}}_{\phi \rho}=\ddot{\mathbf{L}}_{\rho \phi}^{\top} .
\end{aligned}
$$

Logo, é fácil notar que esta matriz é simétrica.

Já a matriz de informação de Fisher $\mathbf{K}_{\theta \theta}$ é definida como

$$
\mathbf{K}_{\theta \theta}=E\left\{-\ddot{\mathbf{L}}_{\theta \theta}\right\}
$$

Na Subseção 3.3.1 apresentamos a matriz de informação observada $-\ddot{\mathbf{L}}_{\theta \theta}$, enquanto que na Subseção 3.3.2 mostramos a matriz de informação de Fisher $\mathbf{K}_{\theta \theta}$.

\subsubsection{Derivação das Hessianas}

Nesta subseção, mostramos como fica dado cada elemento da matriz $\ddot{\mathbf{L}}_{\theta \theta}$. Os cálculos, mais uma vez, são apresentados no Apêndice C. Antes de apresentarmos os resultados, definimos algumas matrizes e vetores que utilizamos. Ressaltamos que algumas dessas quantidades já foram utilizadas anteriormente, apenas apresentamos novamente com o intuito de facilitar o entendimento do leitor.

$$
\mathbf{A}=\left(\begin{array}{rrrrr}
1 & 0 & 0 & \ldots & 0 \\
-\rho & 1 & 0 & \ldots & 0 \\
\vdots & & \vdots & & \vdots \\
0 & \ldots & 0 & -\rho & 1
\end{array}\right)
$$


$\mathbf{B}=\left(\begin{array}{rrrrr}0 & 0 & 0 & \ldots & 0 \\ -1 & 0 & 0 & \ldots & 0 \\ 0 & -1 & 0 & \ldots & 0 \\ \vdots & & \vdots & & \\ 0 & \ldots & 0 & -1 & 0\end{array}\right)$

$\mathbf{D}(\mathbf{v})=\operatorname{diag}\left(v_{1}, \ldots, v_{n}\right), \operatorname{com} v_{i}=-2 W_{g}\left(u_{i}\right)$ e $W_{g}(u)=\frac{g^{\prime}(u)}{g(u)}$,

$\mathbf{u}=\left(u_{1}, \ldots, u_{n}\right)^{\top}$,

$\mathbf{1}_{n}=(1, \ldots, 1)^{\top}$

$\boldsymbol{\epsilon}=\left(\epsilon_{1}, \ldots, \epsilon_{n}\right)^{\top}$

$\mathbf{D}(\mathbf{c})=\operatorname{diag}\left\{c_{1}, \ldots, c_{n}\right\}$, em que $c_{i}=W_{g}^{\prime}\left(u_{i}\right), W_{g}^{\prime}(u)=\mathrm{d} W_{g}(u)=\mathrm{d}(u)$,

$\mathbf{D}(\mathbf{d})=\operatorname{diag}\left\{d_{1}, \ldots, d_{n}\right\}$, em que $d_{i}=u_{i} W_{g}^{\prime}\left(u_{i}\right)$,

$\mathbf{X}$ é a matriz contendo os valores das variáveis explicativas utilizadas de forma paramétrica de todas observações e

$\mathbf{N}$ é uma matriz $q \times q$ chamada de matriz de incidência, formada por valores 0 e 1, definida da mesma forma que no capítulo anterior.

Temos que as submatrizes da diagonal de $\ddot{\mathbf{L}}_{\theta \theta}$ podem ser escritos, em forma matricial, como

$$
\begin{aligned}
\ddot{\mathbf{L}}_{\beta \beta} & =\frac{1}{\phi}\left[(\mathbf{A X})^{\top}(-\mathbf{D}(\mathbf{v})+4 \mathbf{D}(\mathbf{d}))(\mathbf{A X})\right], \\
\ddot{\mathbf{L}}_{f f} & =\frac{1}{\phi}\left[(\mathbf{A N})^{\top}(-\mathbf{D}(\mathbf{v})+4 \mathbf{D}(\mathbf{d}))(\mathbf{A N})\right]-\alpha \mathbf{K}, \\
\ddot{L}_{\phi \phi} & =\frac{1}{\phi^{2}}\left(\frac{n}{2}+\mathbf{u}^{\top} \mathbf{D}(\mathbf{c}) \mathbf{u}-\mathbf{u}^{\top} \mathbf{D}(\mathbf{v}) \mathbf{1}_{n}\right) \mathrm{e} \\
\ddot{L}_{\rho \rho} & =\frac{1}{\phi}\left[(\mathbf{B} \boldsymbol{\epsilon})^{\top}(-\mathbf{D}(\mathbf{v})+4 \mathbf{D}(\mathbf{d}))(\mathbf{B} \boldsymbol{\epsilon})\right] .
\end{aligned}
$$


Já as submatrizes cruzadas podem ser expressas na seguinte forma:

$$
\begin{aligned}
\ddot{\mathbf{L}}_{\beta f} & =\frac{1}{\phi}\left[(\mathbf{A X})^{\top}(-\mathbf{D}(\mathbf{v})+4 \mathbf{D}(\mathbf{d})) \mathbf{A} \mathbf{N}\right] \\
\ddot{\mathbf{L}}_{\beta \phi} & =\frac{1}{\phi^{2}}\left[(\mathbf{A X})^{\top}(-\mathbf{D}(\mathbf{v})+2 \mathbf{D}(\mathbf{d})) \mathbf{A} \boldsymbol{\epsilon}\right] \\
\ddot{\mathbf{L}}_{\beta \rho} & =\frac{1}{\phi}\left[(\mathbf{B} \boldsymbol{\epsilon})^{\top}(\mathbf{D}(\mathbf{v})-4 \mathbf{D}(\mathbf{d})) \mathbf{A X}+(\mathbf{B X})^{\top} \mathbf{D}(\mathbf{v}) \mathbf{A} \boldsymbol{\epsilon}\right], \\
\ddot{\mathbf{L}}_{f \phi} & =\frac{1}{\phi^{2}}\left[(\mathbf{A N})^{\top}(-\mathbf{D}(\mathbf{v})+2 \mathbf{D}(\mathbf{d})) \mathbf{A} \epsilon\right], \\
\ddot{\mathbf{L}}_{f \rho} & =\frac{1}{\phi}\left[(\mathbf{B} \boldsymbol{\epsilon})^{\top}(\mathbf{D}(\mathbf{v})-4 \mathbf{D}(\mathbf{d})) \mathbf{A N}+(\mathbf{B N})^{\top} \mathbf{D}(\mathbf{v}) \mathbf{A} \boldsymbol{\epsilon}\right], \mathrm{e} \\
\ddot{L}_{\rho \phi} & =\frac{1}{\phi^{2}}\left[(\mathbf{B} \boldsymbol{\epsilon})^{\top}(\mathbf{D}(\mathbf{v})-2 \mathbf{D}(\mathbf{d})) \mathbf{A} \boldsymbol{\epsilon}\right]
\end{aligned}
$$

Os elementos restantes da matriz são obtidos pela simetria da matriz.

Com esses cálculos, completamos a matriz observada de Fisher $\left(-\ddot{\mathbf{L}}_{\theta \theta}\right)$. No entanto, toda proposta de estimação neste trabalho será realizada com a matriz de informação de Fisher, que representa a matriz esperada, apresentada na próxima subseção.

\subsubsection{Derivação das informações de Fisher}

A matriz de informação de Fisher penalizada é definida da seguinte forma:

$$
\mathbf{K}_{\theta \theta}=-E\left\{\frac{\partial^{2} L_{p}(\boldsymbol{\theta})}{\partial \boldsymbol{\theta} \partial \boldsymbol{\theta}^{\top}}\right\}
$$

cujas submatrizes são dadas por $\mathbf{K}_{\beta \beta}, \mathbf{K}_{f f}, K_{\phi \phi}, K_{\rho \rho}, \mathbf{K}_{\beta f}, \mathbf{K}_{\beta \phi}, \mathbf{K}_{\beta \rho}, \mathbf{K}_{f \phi}, \mathbf{K}_{f \rho}$ e $K_{\phi \rho}$. Note, mais uma vez, que

$$
\begin{aligned}
& \mathbf{K}_{\beta f}=\mathbf{K}_{f \beta}^{\top}, \mathbf{K}_{\beta \phi}=\mathbf{K}_{\phi \beta}^{\top}, \\
& \mathbf{K}_{\beta \rho}=\mathbf{K}_{\rho \beta}^{\top}, \mathbf{K}_{f \phi}=\mathbf{K}_{\phi f}^{\top}, \\
& \mathbf{K}_{f \rho}=\mathbf{K}_{\rho f}^{\top} \mathrm{e} \mathbf{K}_{\phi \rho}=\mathbf{K}_{\rho \phi}^{\top} .
\end{aligned}
$$

Antes de calcularmos essas matrizes, iremos definir algumas quantidades que serão utilizadas nos cálculos. Assim como no capítulo anterior, definimos 
$f_{g}=E\left\{W^{2}\left(U^{2}\right) U^{4}\right\}$ e $d_{g}=E\left\{W^{2}\left(U^{2}\right) U^{2}\right\}$ com $U \sim S(0,1)$. Também, utilizaremos as condições de regularidade dadas por

$$
\begin{aligned}
& \text { (i) } E\left[\mathbf{U}_{\theta}\right]=\mathbf{0} \quad \text { e } \\
& \text { (ii) }-E\left[\frac{\partial^{2} L_{p}(\boldsymbol{\theta})}{\partial \boldsymbol{\theta} \partial \boldsymbol{\theta}^{\top}}\right]=E\left[\left(\frac{\partial L_{p}(\boldsymbol{\theta})}{\partial \boldsymbol{\theta}}\right)^{2}\right] .
\end{aligned}
$$

Além disso, também precisamos dos dois primeiros momentos da variável $\epsilon_{i}$ presente no modelo. Lembrando que $e_{i} \sim \mathrm{S}(0, \phi)$. Assim obtemos

$$
\begin{array}{r}
\epsilon_{i}=\rho \epsilon_{i-1}+e_{i} \quad \text { com } \epsilon_{0}=0 . \\
\log , \quad \epsilon_{1}=e_{1}, \\
\epsilon_{2}=\rho e_{1}+e_{2}, \\
\epsilon_{3}=\rho\left(\rho e_{1}+e_{2}\right)+e_{3}=\rho^{2} e_{1}+\rho e_{2}+e_{3}, \\
\vdots \\
\epsilon_{i}=\rho^{i-1} e_{1}+\rho^{i-2} e_{2}+\cdots+\rho e_{i-1}+e_{i} .
\end{array}
$$

Pelas propriedades das distribuições simétricas e pela forma que escrevemos a variável $\epsilon_{i}$ e, lembrando que as variáveis $e_{i}$ 's são independentes, facilmente temos que

$$
\begin{aligned}
E\left[\epsilon_{i}\right]=0 \quad \mathrm{e} \\
\operatorname{Var}\left(\epsilon_{i}\right)=\left[\rho^{2(i-1)}+\rho^{2(i-2)}+\cdots+\rho^{2}+1\right] \phi \xi \quad= \\
=\phi \xi \sum_{j=0}^{i-1} \rho^{2 j}=\phi \xi \frac{\left(1-\rho^{2 i}\right)}{\left(1-\rho^{2}\right)}=E\left(\epsilon_{i}^{2}\right) .
\end{aligned}
$$

Além dessas definições, também usamos uma propriedade de variáveis aleatórias independentes e identicamentes distribuídas. Esta propriedade nos diz que a informação fornecida pelo vetor de variáveis $X_{1}, X_{2}, \ldots, X_{r}$ i.i.d. é igual à informação de Fisher fornecida pela variável $X_{1}$ multiplicada por $r$. No entanto, no modelo em que estamos trabalhando, as variáveis aleatórias não são independentes e identicamentes distribuídas. Para usarmos essa propriedade, escreveremos o logaritmo da função de verossimilhança da seguinte forma: 


$$
L(\boldsymbol{\theta})=-\frac{n}{2} \log \phi+\sum_{i=1}^{n} \log \left\{g\left(u_{i}\right)\right\}=\sum_{i=1}^{n} L_{i}(\boldsymbol{\theta})
$$

em que

$$
L_{i}(\boldsymbol{\theta})=-\frac{1}{2} \log \phi+\log \left\{g\left(u_{i}\right)\right\} .
$$

Também podemos notar que $E\left[g\left(U_{i}\right)\right]$ pode ser expressa como $E[g(U)]$, em que novamente temos $U \sim S(0,1)$, visto que pelas propriedades das distribuições simétricas $u_{i} \sim S(0,1)$. Essa relação vale $\forall i$. Logo, podemos usar a propriedade dada acima. Em muitos cálculos, que serão apresentados no Apêndice C, faremos uso dessa propriedade.

Em relação à diagonal principal da matriz de informação de Fisher, utilizando as matrizes definidas nas subseções anteriores, podemos escrevê-la como

$$
\begin{aligned}
\mathbf{K}_{\beta \beta} & =\frac{4}{\phi} d_{g}(\mathbf{A X})^{\top}(\mathbf{A X}), \\
\mathbf{K}_{f f} & =\frac{4}{\phi} d_{g}(\mathbf{A N})^{\top}(\mathbf{A} \mathbf{N})+\alpha \mathbf{K}, \\
K_{\phi \phi} & =\frac{n}{4 \phi^{2}}\left(4 f_{g}-1\right) \mathrm{e} \\
K_{\rho \rho} & =\frac{4 d_{g} \xi}{1-\rho^{2}}\left[(n-1)-\left(\frac{\rho^{2}\left(1-\rho^{2(n-1)}\right)}{1-\rho^{2}}\right)\right] .
\end{aligned}
$$

Já para os elementos da matriz cruzada de Fisher, temos que

$$
\begin{aligned}
\mathbf{K}_{\beta f} & =\frac{1}{\phi} 4 d_{g}\left[(\mathbf{A X})^{\top}(\mathbf{A N})\right] \\
\mathbf{K}_{\phi \beta} & =\mathbf{0} \\
\mathbf{K}_{\rho \beta} & =\mathbf{0} \\
\mathbf{K}_{\phi f} & =\mathbf{0} \\
\mathbf{K}_{\rho f} & =\mathbf{0} \mathrm{e} \\
K_{\phi \rho} & =0
\end{aligned}
$$

Portanto, a matriz de informação de Fisher está completa. Podemos ver que os parâmetros são ortogonais, exceto $\boldsymbol{\beta}$ e f. Esta matriz pode ser visualizada abaixo 


$$
\mathbf{K}_{\theta \theta}=\left(\begin{array}{rrrr}
\mathbf{K}_{\beta \beta} & \mathbf{K}_{f \beta} & \mathbf{0} & \mathbf{0} \\
\mathbf{K}_{\beta f} & \mathbf{K}_{f f} & \mathbf{0} & \mathbf{0} \\
\mathbf{0} & \mathbf{0} & K_{\phi \phi} & 0 \\
\mathbf{0} & \mathbf{0} & 0 & K_{\rho \rho}
\end{array}\right) .
$$

Com todas essas quantidades obtidas, podemos prosseguir para a estimação dos parâmetros do modelo. Um método de estimação será apresentado a seguir.

\subsection{Procedimentos de Estimação}

Há diversas propostas de estimação no contexto de modelos semiparamétricos. Ibacache-Pulgar [2009] descreve alguns desses métodos. Uma proposta que vem sendo utilizada frequentemente é a estimação por meio do pacote GAMLSS (Generalized Additive Models for Location, Scale and Shape) do software R (Rigby e Stasinopoulos [2005]) que utiliza o processo de escore de Fisher para maximizar o logaritmo da verossimilhança penalizada. No entanto, uma das principais suposições dessa rotina é a independência dos erros das diferentes observações, o que não é válido para o modelo que estamos desenvolvendo. Dessa forma, foi preciso criar um algoritmo, que será descrito nesta seção, para estimar os parâmetros do modelo.

O nosso objetivo é encontrar os valores dos parâmetros do modelo que maximizam sua função de verossimilhança penalizada, ou equivalentemente, os valores que maximizam o logaritmo da função de verossimilhança penalizada. Basicamente, a estimativa $(\hat{\boldsymbol{\theta}})$ obtida por meio da verossimilhança penalizada deve satisfazer

$$
L_{\mathrm{p}}(\hat{\boldsymbol{\theta}}, \alpha) \geq \sup _{\boldsymbol{\theta} \in \Theta^{d}} L_{\mathrm{p}}(\boldsymbol{\theta}, \alpha)=\sup _{\beta, \mathbf{f}, \phi, \rho} L_{\mathrm{p}}(\boldsymbol{\theta}, \alpha) .
$$

Para encontrarmos os valores dos parâmetros que maximizam a função acima, desenvolvemos um algoritmo baseado no escore de Fisher e no algoritmo backfitting. De forma geral, o método escore de Fisher tenta obter a solução em que as funções escores calculadas na Seção 3.2 sejam iguais a 0. É possível mostrar que a $(u+1)$-ésima etapa do processo iterativo escore de Fisher para a estimação de $\boldsymbol{\beta}$ e f é igual a

$$
\left(\begin{array}{ll}
\mathbf{K}_{\beta \beta} & \mathbf{K}_{\beta f} \\
\mathbf{K}_{f \beta} & \mathbf{K}_{f f}
\end{array}\right)^{(u)}\left(\begin{array}{c}
\boldsymbol{\beta}^{(u+1)}-\boldsymbol{\beta}^{(u)} \\
\mathbf{f}^{(u+1)}-\mathbf{f}^{(u)}
\end{array}\right)=\left(\begin{array}{c}
\mathbf{U}_{\beta} \\
\mathbf{U}_{f}
\end{array}\right)^{(u)}
$$


Com os valores obtidos anteriormente, podemos escrever a expressão acima como

$$
\begin{array}{rr}
\left(\begin{array}{rr}
\frac{4}{\phi} d_{g}(\mathbf{A X})^{\top}(\mathbf{A X}) & \frac{1}{\phi} 4 d_{g}\left[(\mathbf{A X})^{\top}(\mathbf{A N})\right] \\
\frac{1}{\phi} 4 d_{g}\left[(\mathbf{A N})^{\top}(\mathbf{A X})\right] & \frac{4}{\phi} d_{g}(\mathbf{A N})^{\top}(\mathbf{A N})+\alpha \mathbf{K}
\end{array}\right)^{(u)}\left(\begin{array}{r}
\boldsymbol{\beta}^{(u+1)}-\boldsymbol{\beta}^{(u)} \\
\mathbf{f}^{(u+1)}-\mathbf{f}^{(u)}
\end{array}\right)= \\
\left(\begin{array}{r}
(\mathbf{A X})^{\top} \mathbf{D}(\mathbf{v}) \phi^{-1} \mathbf{A} \boldsymbol{\epsilon} \\
\phi^{-1}(\mathbf{A N})^{\top} \mathbf{D}(\mathbf{v}) \mathbf{A} \boldsymbol{\epsilon}-\alpha \mathbf{K f}
\end{array}\right)^{(u)}
\end{array}
$$

Manipulando algebricamente essas equações, é possível escrevê-las de forma diferente, como a seguir:

$$
\left.\left.\left(\begin{array}{c}
\boldsymbol{\beta}^{(u+1)} \\
\mathbf{f}^{(u+1)}
\end{array}\right)=\left(\begin{array}{c}
\mathbf{S}_{\beta}\left\{\mathbf{r}_{\beta}^{(u, u+1)}+\mathbf{A} \boldsymbol{\mu}^{(u)}\left[\mathbf{I}-\frac{\mathbf{D}(\mathbf{v})}{4 d_{g}}\right.\right. \\
\mathbf{S}_{f}\left\{\mathbf{r}_{f}^{(u, u+1)}+\mathbf{A} \boldsymbol{\mu}^{(u)}\left[\mathbf{I}-\frac{\mathbf{D}(\mathbf{v})}{4 d_{g}}\right]\right.
\end{array}\right]\right\}\right)
$$

em que

$$
\begin{aligned}
& \mathbf{S}_{\beta}=\left[(\mathbf{A X})^{\top}(\mathbf{A X})\right]^{-1}(\mathbf{A X})^{\top} \\
& \mathbf{S}_{f}=\left[\frac{4 d_{g}}{\phi}(\mathbf{A N})^{\top}(\mathbf{A N})+\alpha \mathbf{K}\right]^{-1} \frac{4 d_{g}}{\phi}(\mathbf{A N})^{\top}
\end{aligned}
$$

são matrizes de suavização e

$$
\begin{aligned}
& \mathbf{r}_{\beta}^{(u, u+1)}=\mathbf{A}\left[\left(\frac{\mathbf{D}(\mathbf{v}) \mathbf{y}}{4 d_{g}}\right)^{(u)}-\mathbf{N} \mathbf{f}^{(u+1)}\right] \\
& \mathbf{r}_{f}^{(u, u+1)}=\mathbf{A}\left[\left(\frac{\mathbf{D}(\mathbf{v}) \mathbf{y}}{4 d_{g}}\right)^{(u)}-\mathbf{X} \boldsymbol{\beta}^{(u+1)}\right]
\end{aligned}
$$

são denominados resíduos parciais. Podemos notar que a cada iteração, as estimativas dos parâmetros dependem de sua respectiva matriz de suavização, do resíduo parcial em relação ao outro parâmetro e do vetor de médias, obtidos na iteração anterior. As matrizes utilizadas acima já foram definidas anteriormente. Convém ressaltar também que as estimativas dependem da distribuição simétrica utilizada pela matriz $\mathbf{D}(\mathbf{v})$ e do elemento $d_{g}$. Outro importante parâmetro utilizado é o parâmetro de suavização $\alpha$, que discutiremos adiante sua estimação. 
Os parâmetros $\phi$ e $\rho$ são ortogonais entre si e entre os parâmetros $\boldsymbol{\beta}$ e $\mathbf{f}$. Assim, a estimação de $\phi$ e $\rho$ pode ser feita separadamente, também pelo método escore de Fisher, atualizando as estimativas a cada iteração da seguinte forma:

$$
\begin{aligned}
\phi^{(u+1)} & =\phi^{(u)}+\left\{K_{\phi \phi}^{-1}(\boldsymbol{\theta}) U_{\phi}\right\}^{(u)} \mathrm{e} \\
\rho^{(u+1)} & =\rho^{(u)}+\left\{K_{\rho \rho}^{-1}(\boldsymbol{\theta}) U_{\rho}\right\}^{(u)} .
\end{aligned}
$$

Resolver diretamente o algoritmo escore de Fisher para os parâmetros $\boldsymbol{\beta}$ e f não é aconselhável em modelos parcialmente lineares. Nestes casos é sugerido utilizar o algoritmo backfitting. Maiores detalhes deste método e algumas condições de convergência são apresentados em Ibacache-Pulgar [2009].

Com a discussão apresentada nesta seção, um código em $\mathrm{R}$ foi produzido para a estimação do modelo desenvolvido neste trabalho. Abaixo, apresentamos o passo a passo do algoritmo produzido.

- Passo (i) - forneça estimativas iniciais para os parâmetros $\boldsymbol{\beta}, \mathbf{f}, \phi$ e $\rho$. Podemos iniciar as estimativas com o ajuste produzido pela rotina GAMLSS e com $\rho$ igual a 0 . Alternativamente, podemos utilizar como estimativa inicial para $\rho$ o estimador de momentos obtido com os resíduos do ajuste produzido pela GAMLSS.

- Passo (ii) - para $u=1,2, \ldots$ calcular:

Os valores de $\mathbf{S}_{\beta}^{(u)}$ e de $\mathbf{r}_{\beta}^{(u, u)}$. Com isso obter a estimativa de $\boldsymbol{\beta}^{(u+1)}$.

Com a estimativa de $\boldsymbol{\beta}^{(u+1)}$, obter as quantidades $\mathbf{S}_{f}^{(u)}$ e $\mathbf{r}_{f}^{(u, u+1)}$. Assim, obter a estimativa $\mathbf{f}^{(u+1)}$. Repetir o passo (ii) até a convergência de $\boldsymbol{\beta}$ e de $\mathbf{f}$.

- Passo (iii) - com as estimativas obtidas no passo anterior, atualizamos as estimativas de $\phi$ e de $\rho$ iterativamente até a convergência pelo método de escore de Fisher, descrito anteriormente, em relação a esses parâmetros. Como esses parâmetros não podem variar em toda a reta, utilizamos funções disponíveis no software $\mathrm{R}$ que limitam o espaço de otimização em relação a esses parâmetros.

- Passo (iv) - Com essas estimativas, repetimos os passos (ii) e (iii) como se as estimativas obtidas anteriormente fossem os novos chutes iniciais. Repetimos até que a convergência global seja alcançada. Ou seja, até que os resultados do passo (iv) ${ }^{(k)}$ sejam razoalvemente iguais aos resultados obtidos no passo (iv) $)^{(k-1)}$. 
A condição de convergência utilizada em todos os passos do algoritmo foi que a diferença máxima entre os elementos dos vetores estimados da iteração $(k)$ e da $(k-1)$ fossem menores do que 0,001 .

Por analogia a resultados do algoritmo backfitting, é possível determinar o número efetivo de parâmetros do modelo para a parte não paramétrica. Temos o seguinte resultado:

$$
d f(\alpha)=\operatorname{tr}\left\{(\mathbf{A N}) \mathbf{S}_{f}\right\}=\operatorname{tr}\left\{(\mathbf{A N})\left[\frac{4 d_{g}}{\phi}(\mathbf{A N})^{\top}(\mathbf{A N})+\alpha \mathbf{K}\right]^{-1} \frac{4 d_{g}}{\phi}(\mathbf{A N})^{\top}\right\}
$$

Assim, o número total de graus de liberdade é igual a $2+p+d f(\alpha)$, em que $p$ são os graus de liberdade associados à parte paramétrica, além disso temos os 2 parâmetros referentes a $\phi$ e $\rho$.

\subsubsection{Escolha do parâmetro de suavização}

Como já salientamos anteriormente, o parâmetro $\alpha$ é de suma importância, controlando o trade-off entre um super ajustamento e um modelo viesado. $\mathrm{Na}$ literatura, há algumas formas de estimar esse parâmetro. Naturalmente surgiu a ideia de estimar os parâmetros do modelo para diferentes valores de $\alpha$ e escolher aquele que apresenta o melhor valor em uma determinada medida como, por exemplo, pelo critério de Akaike (AIC).

Outras formas frequentemente utilizadas para a estimação do valor de $\alpha$ são a validação cruzada e a validação cruzada generalizada. A primeira consiste em estimar os parâmetros diversas vezes, cada vez sem uma observação, e verificar quão bem o ajuste se comporta em prever esta observação. Existem alguns resultados algébricos que facilitam o custo computacional para calcular essas medidas. O método de validação cruzada generalizada é uma adaptação da validação cruzada reduzindo a dificuldade computacional. Maiores detalhes desses métodos podem ser encontrados em Ibacache-Pulgar [2009] e Green e Silverman [1994].

De forma geral, a validação cruzada generalizada pode ser escrita como

$$
V C G(\alpha)=\frac{\|\mathbf{y}-\hat{\mathbf{y}}\|^{2}}{\left(1-n^{-1} \operatorname{tr}\{\mathbf{H}(\alpha)\}\right)^{2}},
$$

em que a matriz $\mathbf{H}(\alpha)$ é matriz chapéu, em que $\hat{\mathbf{y}}=\mathbf{H y}$. O valor de $\alpha$ que minimiza $\operatorname{VCG}(\alpha)$ é escolhida como estimativa. Outra possível critério para a 
escolha de $\alpha$ é o uso do critério de Akaike modificado, apresentado em Aydin [2011], que é uma adaptação do AIC para escolha do parâmetro de suavização em modelos não paramétricos. Esta medida é dada por

$$
A I C_{c}(\alpha)=\log \frac{\|\mathbf{y}-\hat{\mathbf{y}}\|^{2}}{n}+1+\frac{2(\operatorname{tr}\{\mathbf{H}(\alpha)\}+1)}{n-2-\operatorname{tr}\{\mathbf{H}(\alpha)\}}
$$

e mais uma vez, escolhemos $\alpha$ que minimize esta medida.

Estes três critérios podem ser utilizados para a escolha do parâmetro de suavização. No Capítulo 5, em que apresentaremos uma aplicação, mostraremos que os três critérios apresentam resultados similares para o caso apresentado.

Nestas duas últimas medidas, é necessário a obtenção da matriz $\mathbf{H}$, portanto mostraremos como calculá-la.

Por meio das funções escores obtidas igualando-as a zero, obtemos as seguintes equações:

$$
\begin{aligned}
\left\{(\mathbf{A X})^{\top} \mathbf{D}(\mathbf{v}) \mathbf{A X}\right\} \boldsymbol{\beta} & =(\mathbf{A X})^{\top} \mathbf{D}(\mathbf{v})(\mathbf{y}-\mathbf{N f}) \\
\left\{(\mathbf{A N})^{\top} \mathbf{D}(\mathbf{v}) \mathbf{A N}+\alpha \phi \mathbf{K}\right\} \mathbf{f} & =(\mathbf{A N})^{\top} \mathbf{D}(\mathbf{v})(\mathbf{y}-\mathbf{X} \boldsymbol{\beta})
\end{aligned}
$$

Manipulando essas equações algebricamente, podemos escrever as estimativas de $\boldsymbol{\beta}$ e $\mathbf{f}$ da seguinte forma:

$$
\begin{aligned}
\hat{\boldsymbol{\beta}} & =\left[(\mathbf{A X})^{\top} \mathbf{W}_{x} \mathbf{A X}\right]^{-1}(\mathbf{A X})^{\top} \mathbf{W}_{x} \mathbf{y} \quad \mathrm{e} \\
\hat{\mathbf{f}} & =\left[(\mathbf{A N})^{\top} \mathbf{W}_{f} \mathbf{A N}+\alpha \phi \mathbf{K}\right]^{-1}(\mathbf{A N})^{\top} \mathbf{W}_{f} \mathbf{y}
\end{aligned}
$$

em que

$$
\begin{aligned}
\mathbf{W}_{x} & =\mathbf{D}(\mathbf{v})-\mathbf{D}(\mathbf{v})(\mathbf{A N})\left[(\mathbf{A N})^{\top} \mathbf{D}(\mathbf{v}) \mathbf{A N}+\alpha \phi \mathbf{K}\right]^{-1}(\mathbf{A N})^{\top} \mathbf{D}(\mathbf{v}) \quad \mathrm{e} \\
\mathbf{W}_{f} & =\mathbf{D}(\mathbf{v})-\mathbf{D}(\mathbf{v})(\mathbf{A X})\left[(\mathbf{A X})^{\top} \mathbf{D}(\mathbf{v}) \mathbf{A X}\right]^{-1}(\mathbf{A X})^{\top} \mathbf{D}(\mathbf{v}) .
\end{aligned}
$$

Como $\hat{y}_{i}=x_{i}^{\top} \hat{\boldsymbol{\beta}}+\hat{f}\left(t_{i}\right)+\hat{\rho}\left(y_{i-1}-x_{i-1}^{\top} \hat{\boldsymbol{\beta}}-\hat{f}\left(t_{i-1}\right)\right)$, podemos escrever esta expressão em forma matricial da seguinte forma

$$
\begin{gathered}
\hat{\mathbf{y}}=\{(\mathbf{A X}) \hat{\boldsymbol{\beta}}+(\mathbf{A N}) \hat{\mathbf{f}}-\hat{\rho} \mathbf{B y}\}= \\
\left\{(\mathbf{A X})\left[(\mathbf{A X})^{\top} \mathbf{W}_{x}(\mathbf{A X})\right]^{-1}(\mathbf{A X})^{\top} \mathbf{W}_{x} \mathbf{A}+\right. \\
\left.(\mathbf{A N})\left[(\mathbf{A N})^{\top} \mathbf{W}_{f}(\mathbf{A N})+\alpha \phi \mathbf{K}\right]^{-1}(\mathbf{A N})^{\top} \mathbf{W}_{x} \mathbf{A}-\hat{\rho} \mathbf{B}\right\} \mathbf{y}
\end{gathered}
$$


Portanto, a matriz chapéu é dada por:

$$
\begin{gathered}
\mathbf{H}=\left\{(\mathbf{A X})\left[(\mathbf{A X})^{\top} \mathbf{W}_{x}(\mathbf{A X})\right]^{-1}(\mathbf{A X})^{\top} \mathbf{W}_{x} \mathbf{A}+\right. \\
\left.(\mathbf{A N})\left[(\mathbf{A N})^{\top} \mathbf{W}_{f}(\mathbf{A N})+\alpha \phi \mathbf{K}\right]^{-1}(\mathbf{A N})^{\top} \mathbf{W}_{x} \mathbf{A}-\hat{\rho} \mathbf{B}\right\}
\end{gathered}
$$

Assim, temos todos os subsídios para o cálculo das medidas comentadas anteriormente.

\subsection{Resultados assintóticos}

Como o modelo parcialmente linear com erros simétricos $\mathrm{AR}(1)$, desenvolvido neste trabalho, trata-se de uma generalização de modelo simétricos, modelos parcialmente lineares e modelos com erros autoregressivos, é razoável supor que sob as condições de regularidade, as estimativas de verossimilhança de $\boldsymbol{\theta}$ são assintoticamente normais. Mais especificamente, podemos supor que $\hat{\boldsymbol{\theta}}$ $\sim N_{p^{*}}\left(\boldsymbol{\theta}, \mathbf{K}_{\theta \theta}^{-1}\right)$, em que $p^{*}$ representa o número total de parâmetros estimados.

Por meio da matriz de informação de Fisher calculada na Seção 3.4 e de propriedades de inversa de matriz em blocos, é possível mostrar que $\mathbf{K}_{\theta \theta}^{-1}$ é dada por

$$
\mathbf{K}_{\theta \theta}^{-1}=\left(\begin{array}{rrrr}
\mathbf{U} & \mathbf{V} & \mathbf{0} & \mathbf{0} \\
\mathbf{Z} & \mathbf{W} & \mathbf{0} & \mathbf{0} \\
\mathbf{0} & \mathbf{0} & \mathbf{K}_{\phi \phi}^{-1} & 0 \\
\mathbf{0} & \mathbf{0} & 0 & \mathbf{K}_{\rho \rho}^{-1}
\end{array}\right)
$$

em que

$$
\begin{gathered}
\mathbf{U}=\left[\mathbf{K}_{\beta \beta}-\mathbf{K}_{f \beta} \mathbf{K}_{f f}^{-1} \mathbf{K}_{\beta f}\right]^{-1}, \\
\mathbf{V}=-\mathbf{K}_{\beta \beta}^{-1} \mathbf{K}_{f \beta}\left(\mathbf{K}_{f f}-\mathbf{K}_{\beta f} \mathbf{K}_{\beta \beta}^{-1} \mathbf{K}_{f \beta}\right)^{-1}, \\
\mathbf{Z}=-\mathbf{K}_{f f}^{-1} \mathbf{K}_{\beta f}\left(\mathbf{K}_{\beta \beta}-\mathbf{K}_{f \beta} \mathbf{K}_{f f}^{-1} \mathbf{K}_{\beta f}\right)^{-1} \mathrm{e} \\
\mathbf{W}=\left[\mathbf{K}_{f f}-\mathbf{K}_{\beta f} \mathbf{K}_{\beta \beta}^{-1} \mathbf{K}_{f \beta}\right]^{-1} .
\end{gathered}
$$

Com esses cálculos, podemos observar que os parâmetros são assintoticamente não correlacionados, com exceção dos parâmetros $\boldsymbol{\beta}$ e dos parâmetros relacionados à parte não paramétrica, que podem apresentar correlação.

Portanto, com os resultados desta seção, podemos produzir inferências sobre as estimativas dos parâmetros do modelo, quando o número de observações 
presentes na modelagem é elevado.

\subsection{Conclusões do Capítulo}

Neste terceiro capítulo, apresentamos a teoria do modelo desenvolvido. Inicialmente, mostramos suas propriedades e sua utilidade. Após esta primeira etapa, desenvolvemos a teoria comumente utilizada na estatística clássica, em que derivamos as funções escore, bem como as matrizes de Fisher observada e esperada. Por fim, apresentamos como este modelo pode ser ajustado por meio do software R. Nos próximos capítulos continuaremos desenvolvendo este modelo, bem como apresentaremos uma aplicação prática. 


\section{Capítulo 4}

\section{Análise de Diagnóstico}

Uma etapa importante da modelagem estatística é a análise de diagnóstico do modelo proposto. $\mathrm{Na}$ análise de dados, geralmente, os modelos de regressão tentam captar relações entre variáveis controlando o efeito de outras variáveis. No entanto, o modelo verdadeiro quase nunca é conhecido na prática, o que faz com que o modelo proposto nem sempre se adeque bem aos dados. Portanto, a análise de diagnóstico do modelo ajustado tem como principal objetivo verificar a sua adequação. Usualmente, procura-se saber se os valores ajustados estão muito longe dos valores reais, para detectar pontos aberrantes, bem como verificar se há afastamentos importantes das suposições do modelo, como por exemplo a distribuição dos erros. Uma outra verificação comumente realizada é a análise de influência das observações, para detectar se alguma observação está influenciando demasiadamente as estimativas dos parâmetros do modelo. Neste sentido, o ajuste é realizado sem a presença de uma ou de um conjunto de observações. Outra técnica mais elaborada e recente de diagnóstico é o método de influência local (Cook [1986]), em que é possível estudar a sensibilidade do ajuste sob pequenas perturbações no modelo ou nos dados.

Neste capítulo, primeiramente na Seção 4.1, apresentamos algumas técnicas de diagnóstico para o caso de regressão linear com erros normais. Já nas próximas seções, iremos generalizar os métodos discutidos para o caso linear normal para o modelo desenvolvido neste trabalho. Vale ressaltar que daremos uma maior atenção para o método de influência local. 


\subsection{Análise de diagnóstico do modelo linear nor- mal}

Como salientamos na introdução anterior, apresentamos algumas técnicas para o caso mais simples, ou seja, para o modelo de regressão linear normal e assim facilitar o entendimento do tema discutido. Após essa apresentação inicial, generalizamos as técnicas para o caso considerado neste trabalho.

Mais especificamente, apresentamos a definição de resíduos, os resíduos padronizados, o conceito de alavanca e o método de influência local. Para introduzir tais técnicas, consideramos o seguinte modelo de regressão:

$$
y_{i}=\beta_{1}+\beta_{2} x_{2 i}+\cdots+\beta_{p} x_{p i}+\epsilon_{i},
$$

com $i=1, \ldots, n$ e $\epsilon_{i}$ 's são variáveis aleatórias independentes normalmente distribuídas de média zero e variância $\sigma^{2}$.

Uma boa referência sobre o assunto é Paula [2010] que além de apresentar os métodos discutidos neste trabalho, também realiza um contexto histórico da análise de diagnóstico, apresentando ainda boas referências sobre as técnicas debatidas.

O $i$-ésimo resíduo do modelo é definido como qualquer função do $i$-ésimo valor observado e do $i$-ésimo valor predito. Usualmente, o resíduo $r_{i}$ é definido como $y_{i}-\hat{\mu}_{i}$, no entanto outras tipos de resíduos são utilizados, como os resíduos absolutos, resíduos quadráticos e resíduos relativos. Com a definição usual, o vetor de resíduos $\mathbf{r}=\left(r_{1}, \ldots, r_{n}\right)^{\top}$ pode ser escrito como $\mathbf{r}=\mathbf{y}-\hat{\boldsymbol{\mu}}=$ $\left(\mathbf{I}_{n}-\mathbf{H}\right) \mathbf{y}$, em que $\mathbf{H}=\mathbf{X}\left(\mathbf{X}^{\top} \mathbf{X}\right)^{-1} \mathbf{X}^{\top}$, visto que, para o modelo acima, $\hat{\boldsymbol{\beta}}=\left(\mathbf{X}^{\top} \mathbf{X}\right)^{-1} \mathbf{X}^{\top} \mathbf{y}, \log 0 \hat{\boldsymbol{\mu}}=\mathbf{X} \hat{\boldsymbol{\beta}}=\mathbf{H y}$.

Através das relações comentadas no paragráfo anterior, é fácil ver que:

$$
\begin{gathered}
E(\mathbf{r})=\left(\mathbf{I}_{n}-\mathbf{H}\right) E(\mathbf{y})=0 \quad \mathrm{e} \\
\operatorname{Var}(\mathbf{r})=\sigma^{2}\left(\mathbf{I}_{n}-\mathbf{H}\right) .
\end{gathered}
$$

Também, torna-se fácil mostrar que a $\operatorname{Cov}\left(r_{i}, r_{j}\right)=-\sigma^{2} h_{i j}$, para $i$ diferente de $j$. Portanto, além de os resíduos não serem independentes, cada resíduo $r_{i}$ apresenta uma distribuição normal de média 0 e variância igual a $\sigma^{2}\left(1-h_{i i}\right)$.

Como esses resíduos não apresentam a mesma variância, dificulta-se a comparação entre eles. Assim, outro tipo de resíduo foi proposto, dividindo o resíduo definido anteriormente pelo seu respectivo desvio padrão, a fim de padronizá-lo e tornar a comparação mais justa. Assim, foi proposto o resíduo 
studentizado, definido como

$$
t_{i}=\frac{r_{i}}{s\left(1-h_{i i}\right)^{\frac{1}{2}}},
$$

em que $s^{2}$ é o erro quadrático médio dos resíduos.

No entanto, pelo fato dos resíduos não serem independentes, a distribuição dos resíduos studentizados não representa uma distribuição t de Student. Para resolver tal problema, foi proposto substituir o valor de $s^{2}$ por $s_{(i)}^{2}$, que é definido como o erro quadrático médio obtido do modelo ajustado sem a $i$ ésima observação.

Portanto, o novo resíduo studentizado é dado por:

$$
t_{i}^{*}=\frac{r_{i}}{s_{(i)}\left(1-h_{i i}\right)^{\frac{1}{2}}} .
$$

É possível mostrar que esse novo resíduo apresenta uma distribuição t de Student com $n-p-1$ graus de liberdade. Também, após algumas operações algébricas, temos que

$$
t_{i}^{*}=t_{i}\left(\frac{n-p-1}{n-p-t_{i}^{2}}\right)^{\frac{1}{2}} .
$$

A matriz chapéu, matriz $\mathbf{H}$, é, também, frequentemente utilizada na análise de diagnóstico. Essa matriz, além de simétrica, é idempotente, logo seu posto é igual ao seu traço, soma dos elementos da diagonal principal, dado por $p$. Como $\hat{\boldsymbol{\mu}}=\mathbf{H y}$, temos que

$$
\hat{y}_{i}=h_{i i} y_{i}+\sum_{i \neq j} h_{j i} y_{j} .
$$

Como $\mathbf{H}$ é idempotente, temos ainda

$$
\sum_{j \neq i} h_{i j}^{2}=h_{i i}\left(1-h_{i i}\right) .
$$

Se $h_{i i}=1$, teremos que $\hat{y}_{i}=y_{i}$. Portanto, quanto maior for o valor de $h_{i i}$, maior é o efeito da própria observação em sua estimativa, o que não é desejável, visto que o objetivo de modelagem é obter um modelo com estimativas resistentes (robustas), ou seja, que funcione não apenas na amostra de desenvolvimento utilizada. A medida $h_{i i}$ é conhecida como a influência da $i$-ésima observação em sua própria estimativa. Assim, um procedimento utilizado fre- 
quentemente é verificar a diagonal principal da matriz $\mathbf{H}$ para detectar valores altos nesses elementos. $\mathrm{O}$ valor de $h_{i i}$ também pode ser interpretado como $\frac{\partial \hat{y}_{i}}{\partial y_{i}}$, logo, representa a variação na estimativa ao mudarmos a observação em um infinitésimo.

Em muitos tipos de modelo, não é conhecido um patamar para compararmos os valores de $h_{i i}$, ou seja, não conseguimos definir o quão alto é cada valor. Nesses casos, costuma-se analisar valores que destoam excessivamente dos demais. Já para o caso que estamos apresentando nesta seção, o modelo normal linear, temos que a média de $h_{i i}$ é dada $\frac{\operatorname{tr}(\mathbf{H})}{n}=\frac{p}{n}$. Assim, geralmente analisamse os valores da diagonal maiores ou iguais do que $\frac{2 p}{n}$, conhecidos como pontos de alavanca. Vale ressaltar que esses pontos geralmente encontram-se em regiões remotas do subespaço formado pelas variáveis explicativas, ou seja, pela matriz X.

Vários métodos de influência das observações nas estimativas do modelo também são utilizados na análise de diagnóstico como, por exemplo, a distância de Cook ou o a medida DFFITS. Maiores detalhes podem ser encontrados em Paula [2010]. Estas medidas avaliam com as estimativas se alteram ao retirarmos uma observação do modelo. Não obstante, outros pesos fixados, a priori, podem ser usados na distância de Cook.

Baseado nos métodos de influência, surgiu um dos mais modernos e elaborados métodos de diagnóstico, a influência local, apresentado por Cook [1986]. Este procedimento nos permite avaliar como uma medida de influência se altera ao realizarmos pequenas modificações no modelo proposto ou nos dados. Assim, podemos verificar se alguma observação ao ser mudada causa alterações significativas nas inferências.

Vamos, agora, formalizar o método de influência local. Denotaremos por $\boldsymbol{\delta}$ um vetor $q \times 1$ de perturbações, podendo ser, por exemplo, um vetor de pesos, em que $q$ pode variar de 1 a $n$, sendo geralmente igual a $n$. Seja $\boldsymbol{\delta}_{0}$ o vetor de não pertubação do modelo, $\mathrm{L}(\boldsymbol{\theta})$ o logaritmo da função de verosimilhança e $\boldsymbol{\theta}$ o vetor de parâmetros. Para verificarmos quanto a varossimilhança se altera ao utilizarmos o vetor de pesos $\boldsymbol{\delta}$, analisaremos o afastamento da verossimilhança (likelihood displacement) definido por

$$
L D(\boldsymbol{\delta})=2\left[L(\hat{\boldsymbol{\theta}})-L\left(\hat{\boldsymbol{\theta}_{\delta}}\right)\right],
$$

em que $\hat{\boldsymbol{\theta}}_{\delta}$ representa a estimativa de verossimilhança do modelo perturbado. Temos que $\operatorname{LD}(\boldsymbol{\delta}) \geq 0$.

A sugestão de Cook é estudar a função $\operatorname{LD}(\boldsymbol{\delta})$ em torno de $\boldsymbol{\delta}_{0}$, o vetor de não perturbação do modelo proposto. Mais especificamente, é sugerido estudar 
a função em torno de uma direção unitária 1 selecionada, ou seja, analisar o comportamento do gráfico de $\operatorname{LD}\left(\boldsymbol{\delta}_{0}+a \mathbf{l}\right)$, denominado gráfico da linha projetada, contra $a$, em que $a$ é um número real. Nota-se que esse gráfico tem um mínimo local no ponto $a=0$. Cada uma dessas linhas projetadas pode ser vista como uma curvatura normal $C_{l}(\boldsymbol{\theta})$, conceito de geometria diferencial, ao redor do 0. Usualmente, utiliza-se a direção $\mathbf{l}_{\max }$, maior curvatura de $C_{l}(\boldsymbol{\theta})$. A direção $\mathbf{l}_{\max }$ nos mostra quais observações podem alterar mais os resultados ao sofrerem uma pequena perturbação. Assim, um gráfico de $\mathbf{l}_{\max }$ contra a ordem das observações é muito utilizado para detectar as observações que apresentam um peso maior nessa direção, isto é, as observações que podem estar "atrapalhando"o modelo.

Para entender essa metodologia, imagine que você está em um ponto do espaço, por exemplo sua casa, cercado por linhas que começam do chão e vão para cima. Cada linha sobe em uma velocidade diferente, de acordo com um ângulo diferente e o seu interesse é detectar qual dessas linhas sobe mais rapidamente. O método de influência local procura esta linha, cuja velocidade de subida indica quanto a distância $\operatorname{LD}(\boldsymbol{\delta})$ se afasta na direção já mencionada.

Cook [1986], através de geometria diferencial, mostra que a curvatura normal na direção 1 é dada por

$$
C_{l}(\boldsymbol{\theta})=2\left|\mathbf{l}^{\top} \boldsymbol{\Delta}^{\top} \ddot{\mathbf{L}}_{\theta \theta}^{-1} \boldsymbol{\Delta} \mathbf{l}\right|,
$$

em que $-\ddot{\mathbf{L}}_{\theta \theta}$ é a matriz de informação observada de Fisher, $\Delta$ é uma matriz $r \times q$ com seus elementos dados por

$$
\Delta_{i j}=\frac{\partial^{2} L(\boldsymbol{\theta} \mid \boldsymbol{\delta})}{\partial \theta_{i} \partial \delta_{j}}
$$

em que esses elementos são avaliados em $\boldsymbol{\theta}=\hat{\boldsymbol{\theta}}$ e $\boldsymbol{\delta}=\boldsymbol{\delta}_{0}$ com $i$ variando de 1 a $r$ e $j$ variando de 1 a $q$.

Pela teoria de álgebra linear, sabe-se que o máximo de $\mathbf{l}^{\top} \mathbf{B l}$ é dado pelo maior autovalor, em módulo, de $\mathbf{B}$. Assim, a curvatura normal $C_{l_{\max }}$ é o maior autovalor de $\boldsymbol{\Delta}^{\top} \ddot{\mathbf{L}}_{\theta \theta}^{-1} \boldsymbol{\Delta}$ e consequentemente $\mathbf{l}_{\text {max }}$ é o autovetor correspondente.

Assim, as observações que apresentam um alto valor na direção máxima, ou seja, em $\mathbf{l}_{\text {max }}$, podem causar alterações significativas nas inferências sob pequenas mudanças nos dados ou no modelo. Quando alguma observação apresenta um valor elevado, costuma-se ajustar o modelo sem a observação para verificar se a observação altera drasticamente os resultados. Se isto, de fato acontecer, um método menos sensível a estas observações pode ser especificado para 
tentar amenizar tal problema.

É importante ressaltar que caso a diferença entre o primeiro e o segundo autovalores não seja grande, deverá ser avaliada, também, a direção que esse segundo autovetor proporciona.

Apesar de parecer um pouco complicado o entendimento inicial da metodologia, percebemos que o seu uso não é díficil. A parte mais complicada do método consiste em obter a matriz $\Delta$.

Lesaffre e Verbeke [1998] e Zhu e Zhang [2004] aconselham a construção também de um gráfico alternativo, o gráfico de $C_{l_{i}}$ contra a ordem das informações, em que $\mathbf{l}_{i}$ é um vetor $n \times 1$ de zeros com um na $i$-ésima posição. Também é sugerido padronizar esses valores, ou seja, $C_{i}=\frac{C_{i}}{\sum_{j=1}^{n} C_{j}}$ e analisar as observações cujas posições correspondentes são maiores do que 2 vezes a média $\operatorname{dos} C_{i}^{\prime} s$.

A metodologia apresentada compreende todo o vetor $\boldsymbol{\theta}$ de parâmetros, no entanto, o interesse de avaliar a influência pode conter apenas um subconjunto de $\boldsymbol{\theta}$, o vetor $\boldsymbol{\theta}_{1}$, em que $\boldsymbol{\theta}=\left(\boldsymbol{\theta}_{1}^{\top}, \boldsymbol{\theta}_{2}^{\top}\right)^{\top}$. Por exemplo, $\boldsymbol{\theta}$ pode ser igual a $\left(\boldsymbol{\beta}, \sigma^{2}\right)$ e querermos apenas avaliar a influência das observações em $\boldsymbol{\beta}$. No caso geral, em que o interesse está apenas em $\boldsymbol{\theta}_{1}$, a curvatura normal na direção $\mathbf{l}$ fica dada por

$$
C_{\mathbf{l}}\left(\boldsymbol{\theta}_{1}\right)=2\left|\mathbf{l}^{\top} \boldsymbol{\Delta}^{\top}\left(\ddot{\mathbf{L}}_{\theta \theta}^{-1}-\mathbf{B}_{1}\right) \boldsymbol{\Delta} \mathbf{l}\right|
$$

em que,

$$
\mathbf{B}_{1}=\left(\begin{array}{cc}
\mathbf{0} & \mathbf{0} \\
\mathbf{0} & \ddot{\mathbf{L}}_{\theta_{2} \theta_{2}}^{-1}
\end{array}\right)
$$

Seguindo a notação, temos que $-\ddot{\mathbf{L}}_{\theta_{2} \theta_{2}}$ é referente à matriz de observação de Fisher para $\boldsymbol{\theta}_{2}$. Portanto, agora, ao invés de calcularmos os autovetores da matriz $\boldsymbol{\Delta}^{\top} \ddot{\mathbf{L}}_{\theta \theta}^{-1} \boldsymbol{\Delta}$, calculamos os da matriz $\boldsymbol{\Delta}^{\top}\left(\ddot{\mathbf{L}}_{\theta \theta}^{-1}-\mathbf{B}_{1}\right) \boldsymbol{\Delta}$. Os gráficos comentados anteriormente podem ser usados para avaliar a influência local em $\boldsymbol{\theta}_{1}$.

É importante deixar claro que podemos perturbar o modelo ou os dados de diversas maneiras, portanto deve-se escolher com cuidado a perturbação a fim de obtermos resultados facilmente interpretáveis.

Como a curvatura normal não é invariante a mudanças uniformes na escala, foi proposta na literatura a curvatura conformal (Poon e Poon [1999]), dada por 


$$
B_{\mathbf{l}}(\boldsymbol{\theta})=\frac{C_{\mathbf{l}}(\boldsymbol{\theta})}{\left\|2 \boldsymbol{\Delta}^{\top} \ddot{\mathbf{L}}_{\theta \theta}^{-1} \Delta\right\|_{F}},
$$

em que $\|.\|_{F}$ representa a norma de Frobenius, isto é, $\|\mathbf{A}\|_{F}=\left\{\operatorname{tr}\left(\mathbf{A}^{\top} \mathbf{A}\right)\right\}^{\frac{1}{2}}$.

Uma aspecto interessante e importante desta curvatura é que, em qualquer direção, seu valor está restrito entre 0 e 1 .

Após esta etapa inicial, da introdução de alguns dos principais métodos utilizados atualmente na análise de diagnóstico, iremos apresentar, nas próximas seções, alguns desses métodos para o modelo aqui desenvolvido.

\subsection{Resíduos}

Nesta seção iremos definir os resíduos padronizados para o modelo aqui estudado. O modelo definido em (3.1) e (3.2) é dado por:

$$
\begin{gathered}
y_{i}=\mathbf{x}_{i}^{\top} \boldsymbol{\beta}+f\left(t_{i}\right)+\epsilon_{i}, \\
\epsilon_{i}=\rho \epsilon_{i-1}+e_{i},
\end{gathered}
$$

com $e_{i}$ sendo proveniente de uma distribuição simétrica, mais precisamente, $e_{i}$ $\sim S(0, \phi)$.

Uma proposta de resíduo para o modelo acima é a seguinte:

$$
r_{i}=y_{i}-\mathbf{x}_{i}^{\top} \hat{\boldsymbol{\beta}}-\hat{f}\left(t_{i}\right)-\hat{\rho} \hat{\epsilon}_{i-1},
$$

para $i=1, \ldots, n$, em que $\hat{\epsilon}_{i-1}=y_{i-1}-\mathbf{x}_{i-1}^{\top} \hat{\boldsymbol{\beta}}-\hat{f}\left(t_{i-1}\right)$ e $\hat{\epsilon}_{0}=0$.

Portanto, para $i=2, \ldots, n$, podemos reescrever $r_{i}$ na forma

$$
r_{i}=\left(y_{i}-\hat{\rho} y_{i-1}\right)-\left(\mathbf{x}_{i}^{\top} \hat{\boldsymbol{\beta}}-\hat{\rho} \mathbf{x}_{i-1}^{\top} \hat{\boldsymbol{\beta}}\right)-\left\{\hat{f}\left(t_{i}\right)-\hat{\rho} \hat{f}\left(t_{i-1}\right)\right\} .
$$

A padronização de $r_{i}$ é bastante complicada, porém, podemos obter uma padronização condicional (dadas as estimativas), obtendo o resíduo padronizado

$$
t_{r_{i}}=\frac{\left[\left(y_{i}-\hat{\rho} y_{i-1}\right)-\left(\mathbf{x}_{i}^{\top} \hat{\boldsymbol{\beta}}-\hat{\rho} \mathbf{x}_{i-1}^{\top} \hat{\boldsymbol{\beta}}\right)-\left\{\hat{f}\left(t_{i}\right)-\hat{\rho} \hat{f}\left(t_{i-1}\right)\right\}\right]}{\sqrt{\hat{\phi} \xi}} .
$$

Podemos construir, com esse resíduo, gráficos normais de probabilidade com banda de confiança simulada, a fim de verificarmos a adequação da distribuição dos erros bem como a presença de pontos aberrantes. Podemos cons- 
truir, também, gráficos de $t_{r_{i}}$ contra os valores ajustados e contra a ordem das observações.

\subsection{Pontos de alavanca}

O intuito desta seção é apresentar a forma de obter o grau de alavanca das observações, bem como de nos permitir avaliar tais quantidades.

Como apresentamos na Seção 4.1, o grau de alavanca é utilizado para detectar se a observação está influenciando muito sua própria estimativa, podendo ser visto como

$$
\frac{\partial \hat{y}}{\partial y}
$$

Wei et al. [1998] discutem a matriz generalizada de pontos de alavanca, que é dada por:

$$
\mathbf{G L}(\hat{\boldsymbol{\theta}})=\left.\left[\mathbf{D}_{\theta}\left(-\ddot{\mathbf{L}}_{\theta \theta}\right)^{-1} \ddot{\mathbf{L}}_{\theta y}\right]\right|_{\boldsymbol{\theta}=\hat{\boldsymbol{\theta}}}
$$

Essa expressão é uma forma geral para obter os graus de alavanca. Seguindo a notação, $-\ddot{\mathbf{L}}_{\theta \theta}$ representa matriz de informação observada de Fisher. Já as matrizes restantes são obtidas da seguinte forma:

$$
\mathbf{D}_{\theta}=\frac{\partial \boldsymbol{\mu}}{\partial \boldsymbol{\theta}^{\top}} \quad \text { e } \quad \ddot{\mathbf{L}}_{\theta y}=\frac{\partial^{2} L(\boldsymbol{\theta} ; \mathbf{y})}{\partial \boldsymbol{\theta} \partial \mathbf{y}^{\top}}
$$

Para obtermos a matriz generalizada de pontos de alavanca, definida acima, temos que obter as matrizes $\mathbf{D}_{\theta}$ e $\ddot{\mathbf{L}}_{\theta y}$, visto que a informação observada de Fisher já foi encontrada no capítulo anterior. Primeiramente, apresentaremos os cálculos para a obtenção da matriz $\mathbf{D}_{\theta}$.

Com a Seção 3.1 temos que

$$
\mu_{k}=E\left[y_{k}\right]=\mathbf{x}_{k}^{\top} \boldsymbol{\beta}+\boldsymbol{\eta}_{k}^{\top} \mathbf{f}=>\boldsymbol{\mu}=\mathbf{X} \boldsymbol{\beta}+\mathbf{N f} .
$$

Ainda, a matriz $\mathbf{D}_{\theta}$ pode ser visualizada como

$$
\mathbf{D}_{\theta}=\frac{\partial \boldsymbol{\mu}}{\partial \boldsymbol{\theta}^{\top}}=\left[\left(\frac{\partial \boldsymbol{\mu}}{\partial \boldsymbol{\beta}^{\top}}\right)^{\top},\left(\frac{\partial \boldsymbol{\mu}}{\partial \mathbf{f}^{\top}}\right)^{\top}, \frac{\partial \boldsymbol{\mu}}{\partial \rho}, \frac{\partial \boldsymbol{\mu}}{\partial \phi}\right]^{\top}
$$

Pela equação obtida para $\boldsymbol{\mu}$, temos que: 


$$
\frac{\partial \boldsymbol{\mu}}{\partial \boldsymbol{\beta}^{\top}}=\frac{\partial(\mathbf{X} \boldsymbol{\beta}+\mathbf{N f})}{\partial \boldsymbol{\beta}^{\top}}=\mathbf{X} .
$$

Da mesma forma, é trivial encontrar as derivadas parciais restantes:

$$
\begin{aligned}
\frac{\partial \boldsymbol{\mu}}{\partial \mathbf{f}^{\top}} & =\mathbf{N} \\
\frac{\partial \boldsymbol{\mu}}{\partial \rho} & =0 \mathrm{e} \\
\frac{\partial \boldsymbol{\mu}}{\partial \phi} & =0 .
\end{aligned}
$$

Portanto, obtemos

$$
\mathbf{D}_{\theta}=\left(\mathbf{X}^{\top}, \mathbf{N}^{\top}, 0,0\right)^{\top}
$$

Resta, agora, obter a matriz $\ddot{\mathbf{L}}_{\theta y}$. Para tanto, já obtemos no capítulo anterior a função $\frac{\partial L(\boldsymbol{\theta} ; \mathbf{y})}{\partial \boldsymbol{\theta}}$, dada por $\left[\mathbf{U}_{\beta}^{\top}, \mathbf{U}_{f}^{\top}, U_{\phi}, U_{\rho}\right]^{\top}$. Logo, temos apenas que derivar essa função em relação a y. Para facilitar a organização dos cálculos, escrevemos a matriz procurada da seguinte forma:

$$
\ddot{\mathbf{L}}_{\theta y}=\left[\ddot{\mathbf{L}}_{\beta y}^{\top}, \ddot{\mathbf{L}}_{f y}^{\top}, \ddot{\mathbf{L}}_{\phi y}^{\top}, \ddot{\mathbf{L}}_{\rho y}^{\top}\right]^{\top} \text {. }
$$

Os cálculos para a obtenção da matriz de alavancas estão no Apêndice D. Por meio deles encontramos

$$
\begin{aligned}
& \ddot{\mathbf{L}}_{\beta y}=-\frac{4}{\phi} \mathbf{D}(\mathbf{d}) \mathbf{A X}+\frac{1}{\phi} \mathbf{D}(\mathbf{v}) \mathbf{A X}-\mathbf{B}^{\top}\left[\frac{4 \rho}{\phi} \mathbf{D}(\mathbf{d}) \mathbf{A X}\right]+\mathbf{B}^{\top}\left[\frac{\rho}{\phi} \mathbf{D}(\mathbf{v}) \mathbf{A X}\right], \\
& \ddot{\mathbf{L}}_{f y}=-\frac{4}{\phi} \mathbf{D}(\mathbf{d}) \mathbf{A} \mathbf{N}+\frac{1}{\phi} \mathbf{D}(\mathbf{v}) \mathbf{A} \mathbf{N}-\mathbf{B}^{\top}\left[\frac{4 \rho}{\phi} \mathbf{D}(\mathbf{d}) \mathbf{A} \mathbf{N}\right]+\mathbf{B}^{\top}\left[\frac{\rho}{\phi} \mathbf{D}(\mathbf{v}) \mathbf{A N}\right], \\
& \ddot{\mathbf{L}}_{\phi y}=-\frac{2}{\phi^{2}} \mathbf{D}(\mathbf{d}) \mathbf{A} \boldsymbol{\epsilon}+\frac{1}{\phi^{2}} \mathbf{D}(\mathbf{v}) \mathbf{A} \boldsymbol{\epsilon}-\mathbf{B}^{\top}\left[\frac{2 \rho}{\phi^{2}} \mathbf{D}(\mathbf{d}) \mathbf{A} \boldsymbol{\epsilon}\right]+\mathbf{B}^{\top}\left[\frac{\rho}{\phi^{2}} \mathbf{D}(\mathbf{v}) \mathbf{A} \boldsymbol{\epsilon}\right] \mathrm{e} \\
& \ddot{\mathbf{L}}_{\rho y}=\frac{4}{\phi} \mathbf{D}(\mathbf{d}) \mathbf{B} \boldsymbol{\epsilon}-\frac{1}{\phi} \mathbf{D}(\mathbf{v}) \mathbf{B} \boldsymbol{\epsilon}+\mathbf{B}^{\top}\left[\frac{4 \rho}{\phi} \mathbf{D}(\mathbf{d}) \mathbf{B} \boldsymbol{\epsilon}\right]-\mathbf{B}^{\top}\left[\frac{1}{\phi} \mathbf{D}(\mathbf{v}) \mathbf{C} \boldsymbol{\epsilon}\right],
\end{aligned}
$$

em que 
$\mathbf{C}=\left(\begin{array}{rrrrr}1 & 0 & 0 & \ldots & 0 \\ -2 \rho & 1 & 0 & \ldots & 0 \\ \vdots & & \vdots & & \vdots \\ 0 & \ldots & 0 & -2 \rho & 1\end{array}\right)$

Com as matrizes definidas acima, conseguimos encontrar a matriz de alavancas e assim a utilizarmos na prática para detectar observações que possam estar influenciando desproporcionalmente o próprio valor ajustado.

Na próxima seção, introduziremos o método de influência local para o modelo desenvolvido neste trabalho.

\subsection{Influência local}

O objetivo dessa seção é apresentar a matriz $\boldsymbol{\Delta}$, para avaliarmos a influência local das observações em três esquemas de perturbação diferentes. Com a obtenção dessas matrizes, é possível encontrar as matrizes de curvatura normal que será diagonalizada para encontrarmos o vetor $\mathbf{l}_{\text {max }}$. Este representa a maior curvatura da matriz $\mathbf{C}_{l}(\boldsymbol{\theta})$ e cada valor desse vetor pode ser interpretado como quanto cada observação muda as inferências sob pequenas mudanças no modelo ou nos dados.

Neste trabalho, consideramos três esquemas de perturbações diferentes. No primeiro, consideramos que a variável resposta seja deslocada de um delta. No segundo, consideramos que uma das variáveis explicativas que estão sendo usadas de modo paramétrico sofra tal delta. Por fim, no terceiro esquema, assumimos que cada observação tenha um peso diferente no processo de estimação. Chamaremos cada esquema, respectivamente, de perturbaçao aditiva na resposta do modelo, perturbação aditiva em uma variável contínua do modelo e de ponderação de casos. Apresentamos cada um desses três esquemas em três subseções diferentes.

Lembrando que

$$
\mathbf{C}_{l}(\boldsymbol{\theta})=2\left|\mathbf{l}^{\top} \boldsymbol{\Delta}^{\top} \ddot{\mathbf{L}}_{\theta \theta}^{-1} \boldsymbol{\Delta} \mathbf{l}\right|,
$$

em que $-\ddot{\mathbf{L}}_{\theta \theta}$ é a matriz de informação observada de Fisher, $\Delta$ é uma matriz $r \times q$ com seus elementos dados por

$$
\boldsymbol{\Delta}_{i j}=\frac{\partial^{2} L(\boldsymbol{\theta} \mid \delta)}{\partial \theta_{i} \partial \delta_{j}}
$$


Ressaltamos que nesta seção, denotamos $\boldsymbol{\delta}$ de $\mathbf{w}$, notação mais comumente utilizada em análise de influência local. Também temos que a matriz $\boldsymbol{\Delta}$ é a avaliada sob $\hat{\boldsymbol{\theta}}$ e sob o vetor de não perturbação.

A curvatura conformal também pode ser calculada por meio destas matrizes.

\subsubsection{Perturbação aditiva na resposta do modelo}

Este esquema de perturbação supõe que a variável resposta seja acrescida de um delta. Assim, consideramos que $y_{i w}=y_{i}+w_{i}$. Com isso, podemos avaliar a sensibilidade das estimativas quando introduzimos leves mudanças nas respostas das observações.

Utilizando este esquema de perturbação, temos que

$$
\begin{aligned}
\epsilon_{w i} & =y_{i}+w_{i}-\mathbf{x}_{i}^{\top} \boldsymbol{\beta}-\boldsymbol{\eta}_{i}^{\top} \mathbf{f} \mathrm{e} \\
u_{w i} & =\frac{\left(\epsilon_{w i}-\rho \epsilon_{w i-1}\right)^{2}}{\phi} .
\end{aligned}
$$

Os cálculos para a obtenção da matriz $\Delta$ estão presentes no Apêndice D.

Ressaltamos que a matriz $\boldsymbol{\Delta}$ pode ser decomposta na forma

$$
\left[\left(\frac{\partial \mathbf{U}_{\beta}}{\partial \mathbf{w}}\right)^{\top},\left(\frac{\partial \mathbf{U}_{f}}{\partial \mathbf{w}}\right)^{\top},\left(\frac{\partial U_{\phi}}{\partial \mathbf{w}}\right)^{\top},\left(\frac{\partial U_{\rho}}{\partial \mathbf{w}}\right)^{\top}\right]^{\top},
$$

em que essas medidas, para esse esquema e avaliadas em $\hat{\boldsymbol{\theta}}$ e sob o vetor de não perturbação $\left(\mathbf{w}_{0}=(0, \ldots, 0)^{\top}\right)$, são dadas por

$$
\begin{aligned}
\frac{\partial \mathbf{U}_{\beta}}{\partial \mathbf{w}} & =-\frac{4}{\hat{\phi}} \mathbf{D}(\hat{\mathbf{d}}) \hat{\mathbf{A}} \mathbf{X}+\frac{2}{\hat{\phi}} \mathbf{D}(\hat{\mathbf{v}}) \hat{\mathbf{A}} \mathbf{X}-\mathbf{B}^{\top}\left[\frac{4 \hat{\rho}}{\hat{\phi}} \mathbf{D}(\hat{\mathbf{d}}) \hat{\mathbf{A}} \mathbf{X}\right]+\mathbf{B}^{\top}\left[\frac{2 \hat{\rho}}{\hat{\phi}} \mathbf{D}(\hat{\mathbf{v}}) \hat{\mathbf{A}} \mathbf{X}\right] \\
\frac{\partial \mathbf{U}_{f}}{\partial \mathbf{w}} & =-\frac{4}{\hat{\phi}} \mathbf{D}(\hat{\mathbf{d}}) \hat{\mathbf{A}} \mathbf{N}+\frac{2}{\hat{\phi}} \mathbf{D}(\hat{\mathbf{v}}) \hat{\mathbf{A}} \mathbf{N}-\mathbf{B}^{\top}\left[\frac{4 \hat{\rho}}{\hat{\phi}} \mathbf{D}(\hat{\mathbf{d}}) \hat{\mathbf{A}} \mathbf{N}\right]+\mathbf{B}^{\top}\left[\frac{2 \hat{\rho}}{\hat{\phi}} \mathbf{D}(\hat{\mathbf{v}}) \hat{\mathbf{A}} \mathbf{N}\right], \\
\frac{\partial U_{\phi}}{\partial \mathbf{w}} & =-\frac{2}{\hat{\phi}^{2}} \mathbf{D}(\hat{\mathbf{d}}) \hat{\mathbf{A}} \hat{\boldsymbol{\epsilon}}+\frac{1}{\hat{\phi}^{2}} \mathbf{D}(\hat{\mathbf{v}}) \hat{\mathbf{A}} \hat{\boldsymbol{\epsilon}}-\mathbf{B}^{\top}\left[\frac{2 \hat{\rho}}{\hat{\phi}^{2}} \mathbf{D}(\hat{\mathbf{d}}) \hat{\mathbf{A}} \hat{\boldsymbol{\epsilon}}\right]+\mathbf{B}^{\top}\left[\frac{\hat{\rho}}{\hat{\phi}^{2}} \mathbf{D}(\hat{\mathbf{v}}) \hat{\mathbf{A}} \hat{\boldsymbol{\epsilon}}\right] \\
\frac{\partial U_{\rho}}{\partial \mathbf{w}} & =-\frac{4}{\hat{\phi}} \mathbf{D}(\hat{\mathbf{d}}) \mathbf{B} \hat{\boldsymbol{\epsilon}}+\frac{1}{\hat{\phi}} \mathbf{D}(\hat{\mathbf{v}}) \mathbf{B} \hat{\boldsymbol{\epsilon}}-\mathbf{B}^{\top}\left[\frac{4 \hat{\rho}}{\hat{\phi}} \mathbf{D}(\hat{\mathbf{d}}) \mathbf{B} \hat{\boldsymbol{\epsilon}}\right]-\mathbf{B}^{\top}\left[\frac{\hat{\rho}}{\hat{\phi}} \mathbf{D}(\hat{\mathbf{v}}) \hat{\mathbf{C}} \hat{\boldsymbol{\epsilon}}\right]
\end{aligned}
$$


em que as matrizes $\mathbf{D}(\hat{\mathbf{d}})$ e $\mathbf{D}(\hat{\mathbf{v}})$ são iguais às matrizes $\mathbf{D}(\mathbf{d})$ e $\mathbf{D}(\mathbf{v})$, utilizando apenas as estimativas $\hat{\boldsymbol{\theta}}$. Além disso,

$$
\begin{aligned}
\hat{\boldsymbol{\epsilon}} & =\mathbf{y}-\mathbf{X}^{\top} \hat{\boldsymbol{\beta}}-\mathbf{N}^{\top} \hat{\mathbf{f}} \\
\hat{u}_{i} & =\frac{\left(\hat{\epsilon}_{i}-\hat{\rho} \hat{\epsilon}_{i-1}\right)^{2}}{\hat{\phi}}, i=1, \ldots, n, \quad \text { e } \quad \hat{\epsilon}_{0}=0 .
\end{aligned}
$$

Portanto, está definida a matriz $\Delta$ e, consequentemente, a matriz de curvatura normal. Estas medidas podem ser facilmente implementadas em qualquer software de programação.

\subsubsection{Perturbação aditiva em uma variável contínua do modelo}

Para este esquema, consideramos que a $r$-ésima variável explicativa seja acrescido de delta ao invés da variável resposta. Lembrando que essa variável explicativa tem que ser contínua para a realização dessa perturbação. Portanto, supomos que a $r$-ésima variável explicativa fique expressa por $x_{w i}^{(r)}=x_{i}^{(r)}+w_{i}$, $\forall i=1, \ldots, n$. Para esse caso, temos as seguintes quantidades:

$$
\begin{aligned}
\epsilon_{w i} & =y_{i}-\mathbf{x}_{i}^{\top} \boldsymbol{\beta}-\boldsymbol{\eta}_{i}^{\top} \mathbf{f}-w_{i} \beta_{r} \quad \mathrm{e} \\
u_{w i} & =\frac{\left(\epsilon_{w i}-\rho \epsilon_{w i-1}\right)^{2}}{\phi} .
\end{aligned}
$$

Com os cálculos do Apêndice $\mathrm{D}$, podemos ver que a matriz $\boldsymbol{\Delta}$, avaliada em $\hat{\boldsymbol{\theta}}$ e $\mathbf{w}_{0}$, apresenta as seguintes quantidades 


$$
\begin{aligned}
\frac{\partial \mathbf{U}_{\beta}}{\partial \mathbf{w}} & =-\frac{4 \hat{\beta}_{r}}{\hat{\phi}} \mathbf{D}(\hat{\mathbf{d}}) \hat{\mathbf{A}} \mathbf{X}+\frac{\hat{\beta}_{r}}{\hat{\phi}} \mathbf{D}(\hat{\mathbf{v}}) \hat{\mathbf{A}} \mathbf{X}+\mathbf{B}^{\top}\left[\frac{4 \hat{\rho} \hat{\beta}_{r}}{\hat{\phi}} \mathbf{D}(\hat{\mathbf{d}}) \hat{\mathbf{A}} \mathbf{X}\right]-\mathbf{B}^{\top}\left[\frac{\hat{\rho} \hat{\beta}_{r}}{\hat{\phi}} \mathbf{D}(\hat{\mathbf{v}}) \hat{\mathbf{A}} \mathbf{X}\right], \\
\frac{\partial \mathbf{U}_{f}}{\partial \mathbf{w}} & =-\frac{4 \hat{\beta}_{r}}{\hat{\phi}} \mathbf{D}(\hat{\mathbf{d}}) \hat{\mathbf{A}} \mathbf{N}+\frac{\hat{\beta}_{r}}{\hat{\phi}} \mathbf{D}(\hat{\mathbf{v}}) \hat{\mathbf{A}} \mathbf{N}-\mathbf{B}^{\top}\left[\frac{4 \hat{\rho} \hat{\beta}_{r}}{\hat{\phi}} \mathbf{D}(\hat{\mathbf{d}}) \hat{\mathbf{A}} \mathbf{N}\right]+\mathbf{B}^{\top}\left[\frac{\hat{\rho} \hat{\beta_{r}}}{\hat{\phi}} \mathbf{D}(\hat{\mathbf{v}}) \hat{\mathbf{A}} \mathbf{N}\right], \\
\frac{\partial U_{\phi}}{\partial \mathbf{w}} & =-\frac{2 \hat{\beta}_{r}}{\hat{\phi}^{2}} \mathbf{D}(\hat{\mathbf{d}}) \hat{\mathbf{A}} \hat{\boldsymbol{\epsilon}}+\frac{\hat{\beta}_{r}}{\hat{\phi}^{2}} \mathbf{D}(\hat{\mathbf{v}}) \hat{\mathbf{A}} \hat{\boldsymbol{\epsilon}}-\mathbf{B}^{\top}\left[\frac{2 \hat{\rho} \hat{\beta}_{r}}{\hat{\phi}^{2}} \mathbf{D}(\hat{\mathbf{d}}) \hat{\mathbf{A}} \hat{\boldsymbol{\epsilon}}\right]+\mathbf{B}^{\top}\left[\frac{\hat{\rho} \hat{\beta}_{r}}{\hat{\phi}^{2}} \mathbf{D}(\hat{\mathbf{v}}) \hat{\mathbf{A}} \hat{\boldsymbol{\epsilon}}\right] \mathrm{e} \\
\frac{\partial U_{\rho}}{\partial \mathbf{w}} & =-\frac{4 \hat{\beta}_{r}}{\hat{\phi}} \mathbf{D}(\hat{\mathbf{d}}) \mathbf{B} \hat{\boldsymbol{\epsilon}}+\frac{\hat{\beta}_{r}}{\hat{\phi}} \mathbf{D}(\hat{\mathbf{v}}) \mathbf{B} \hat{\boldsymbol{\epsilon}}-\mathbf{B}^{\top}\left[\frac{4 \hat{\rho} \hat{\beta_{r}}}{\hat{\phi}} \mathbf{D}(\hat{\mathbf{d}}) \mathbf{B} \hat{\boldsymbol{\epsilon}}\right]-\mathbf{B}^{\top}\left[\frac{\hat{\rho} \hat{\beta_{r}}}{\hat{\phi}} \mathbf{D}(\hat{\mathbf{v}}) \hat{\mathbf{C} \hat{\boldsymbol{\epsilon}}]}\right]
\end{aligned}
$$

em que as matrizes $\mathbf{D}(\hat{\mathbf{d}})$ e $\mathbf{D}(\hat{\mathbf{v}})$ são avaliadas em $\hat{\boldsymbol{\theta}}$. Novamente, sob $\hat{\boldsymbol{\theta}}$ e $\mathbf{w}_{0}$, temos

$$
\begin{aligned}
\hat{\boldsymbol{\epsilon}} & =\mathbf{y}-\mathbf{X}^{\top} \hat{\boldsymbol{\beta}}-\mathbf{N}^{\top} \hat{\mathbf{f}} \quad \mathrm{e} \\
\hat{u}_{i} & =\frac{\left(\hat{\epsilon}_{i}-\hat{\rho} \hat{\epsilon}_{i-1}\right)^{2}}{\hat{\phi}}, i=1, \ldots, n, \quad \text { e } \quad \hat{\epsilon}_{0}=0
\end{aligned}
$$

Este esquema também pode ser facilmente implementado.

\subsubsection{Ponderação de casos}

Por fim, consideramos que cada observação possui um peso diferente no processo de estimação, assim podemos avaliar a contribuição individual de cada observação. Este esquema é um dos mais utilizados na prática. Neste caso, o logaritmo da verossimilhança penalizada é dado por

$$
L_{p}(\boldsymbol{\theta} \mid \mathbf{w})=\sum_{i=1}^{n} w_{i} L_{i}(\boldsymbol{\theta})-\frac{\alpha}{2} \mathbf{f}^{\top} \mathbf{K} \mathbf{f},
$$

em que $L_{i}(\boldsymbol{\theta})=-\frac{1}{2} \log \phi+\log \left\{\mathrm{g}\left(\mathrm{u}_{\mathrm{i}}\right)\right\}$ e $\mathbf{w}=\left(w_{1}, \ldots, w_{n}\right)^{\top}$ é o vetor de pesos, com $0 \leq w_{i} \leq 1$. Para este esquema de perturbação, o vetor de não perturbação é $\mathbf{w}_{0}=(1, \ldots, 1)^{\top}$

Através do Apêndice $\mathrm{D}$, temos que os elementos da matriz $\boldsymbol{\Delta}$, avaliados em $\hat{\boldsymbol{\theta}}$ e $\mathbf{w}_{0}$, são dados por 


$$
\begin{aligned}
\frac{\partial \mathbf{U}_{\beta}}{\partial \mathbf{w}} & =(\hat{\mathbf{A}} \mathbf{X})^{\top} \frac{\mathbf{D}(\hat{\mathbf{v}})}{\hat{\phi}} \mathbf{D}\left(\hat{\mathbf{A}}_{\epsilon}\right), \\
\frac{\partial \mathbf{U}_{f}}{\partial \mathbf{w}} & =(\hat{\mathbf{A}} \mathbf{N})^{\top} \frac{\mathbf{D}(\hat{\mathbf{v}})}{\hat{\phi}} \mathbf{D}\left(\hat{\mathbf{A}}_{\epsilon}\right), \\
\frac{\partial U_{\phi}}{\partial \mathbf{w}} & =-\frac{1}{2 \hat{\phi}} \mathbf{1}_{n}-\frac{\mathbf{D}(\hat{\mathbf{d}})}{\hat{\phi}} \mathbf{1}_{n} \quad \mathrm{e} \\
\frac{\partial U_{\rho}}{\partial \mathbf{w}} & =-\left((\mathbf{B} \hat{\boldsymbol{\epsilon}})^{\top} \frac{\mathbf{D}(\hat{\mathbf{v}})}{\hat{\phi}} \mathbf{D}\left(\hat{\mathbf{A}}_{\epsilon}\right)\right)^{\top},
\end{aligned}
$$

em que $\mathbf{1}_{n}=(1, \ldots, 1)^{\top}$ e $\left(\mathbf{D}\left(\hat{\mathbf{A}}_{\epsilon}\right)\right)$ é uma matriz diagonal com elementos dados por $\hat{\mathbf{A}} \hat{\boldsymbol{\epsilon}}$. Lembrando que, assim como para os outros casos, as matrizes sobrescritas com o acento circunflexo devem ser avaliadas em $\hat{\boldsymbol{\theta}}$.

\subsection{Conclusões do capítulo}

Neste capítulo, primeiramente introduzimos as principais técnicas de diagnóstico, exemplificando por meio do modelo de regressão linear normal. Após esta breve revisão, estendemos as técnicas apresentadas para o modelo estudado neste trabalho. Enfatizamos a técnica de influência local, visto que se trata de uma técnica muito flexível e que está sendo amplamente utilizada. No entanto, vários outros esquemas de perturbação, além dos apresentados, podem ser implantados.

No próximo capítulo, onde aplicamos o modelo desenvolvido neste trabalho, também realizaremos análise de diagnóstico, o que tornará clara a utilidade de tais métodos. 


\section{Capítulo 5}

\section{Aplicações}

Neste capítulo, apresentamos uma aplicação prática das teorias desenvolvidas nos capítulos anteriores. Mais precisamente, tentamos analisar a série de dados de temperaturas de duas cidades, apresentadas no exemplo ilustrativo do Capítulo 1. Na primeira seção, explicaremos com mais detalhes a proveniência dos dados e os objetivos das inferências. Após esta etapa, desenvolveremos, nas próximas seções, diferentes tentativas de analisar os dados.

Os códigos em $\mathrm{R}$ criados para a estimação dos parâmetros do modelo, bem como para construir a análise dos resíduos estão disponíveis na página do professor Gilberto Alvarenga Paula (http://www.ime.usp.br/ giapaula).

\subsection{Séries de temperaturas}

O conjunto de dados analisado representa duas séries de temperaturas, medidas em duas diferentes cidades, mais precisamente, são temperaturas registradas em graus Celsius em Ubatuba e Cananéia.

Estas séries foram utilizadas também por Morettin e Toloi [2006] e estão disponíveis para o público no site do professor Morettin do IME-USP (http://www.ime.usp.br/ pam/ST.html).

Descrevendo um pouco melhor este conjunto de dados, temos temperaturas médias mensais das duas cidades, de janeiro de 1976 a dezembro de 1985, isto é, temos 120 observações para cada série. O objetivo desta primeira análise é relacionar as temperaturas de Ubatuba com as temperaturas de Cananéia. Uma primeira possível abordagem para estudar o comportamento das temperaturas de Ubatuba seria utilizar métodos usuais de séries temporais. No entanto, estudaremos a relação entre as duas séries de temperaturas. Mostraremos, ainda, 
algumas análises iniciais que justificam o uso do modelo desenvolvido neste trabalho.

Antes de começarmos o processo de modelagem, apresentaremos brevemente uma análise dos dados. Podemos observar o gráfico de dispersão entre as duas séries de temperaturas na Figura 5.1.

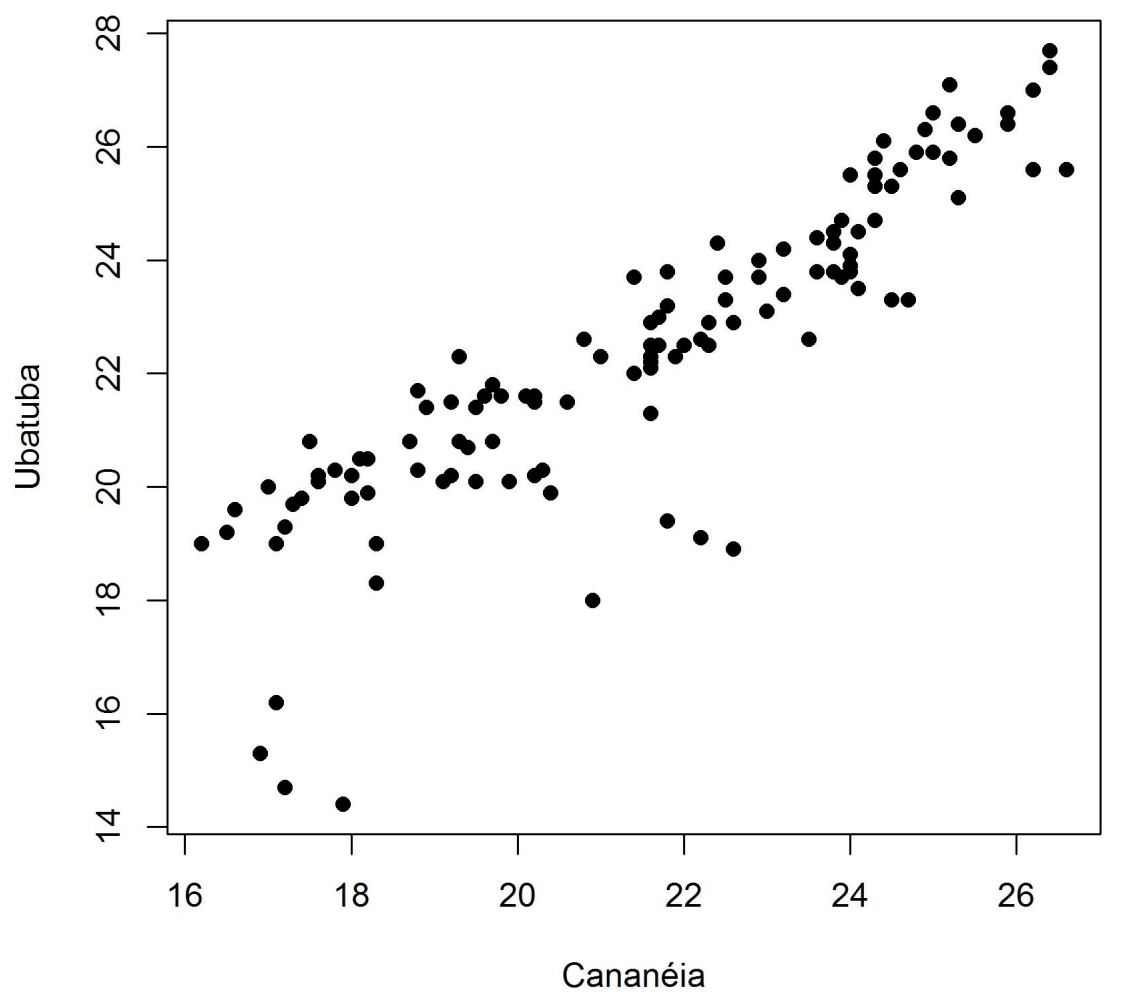

Figura 5.1: Gráfico de dispersão entre as temperaturas médias mensais de Ubatuba e Cananéia.

Com este gráfico, observamos claramente que há uma forte relação entre as temperaturas nas cidades estudadas. Portanto, é plausível a construção de um modelo relacionando as duas temperaturas. Além desta clara relação, é possível imaginar que a temperatura de Ubatuda também esteja relacionada com o tempo, apresentando até mesmos ciclos de temperaturas. 


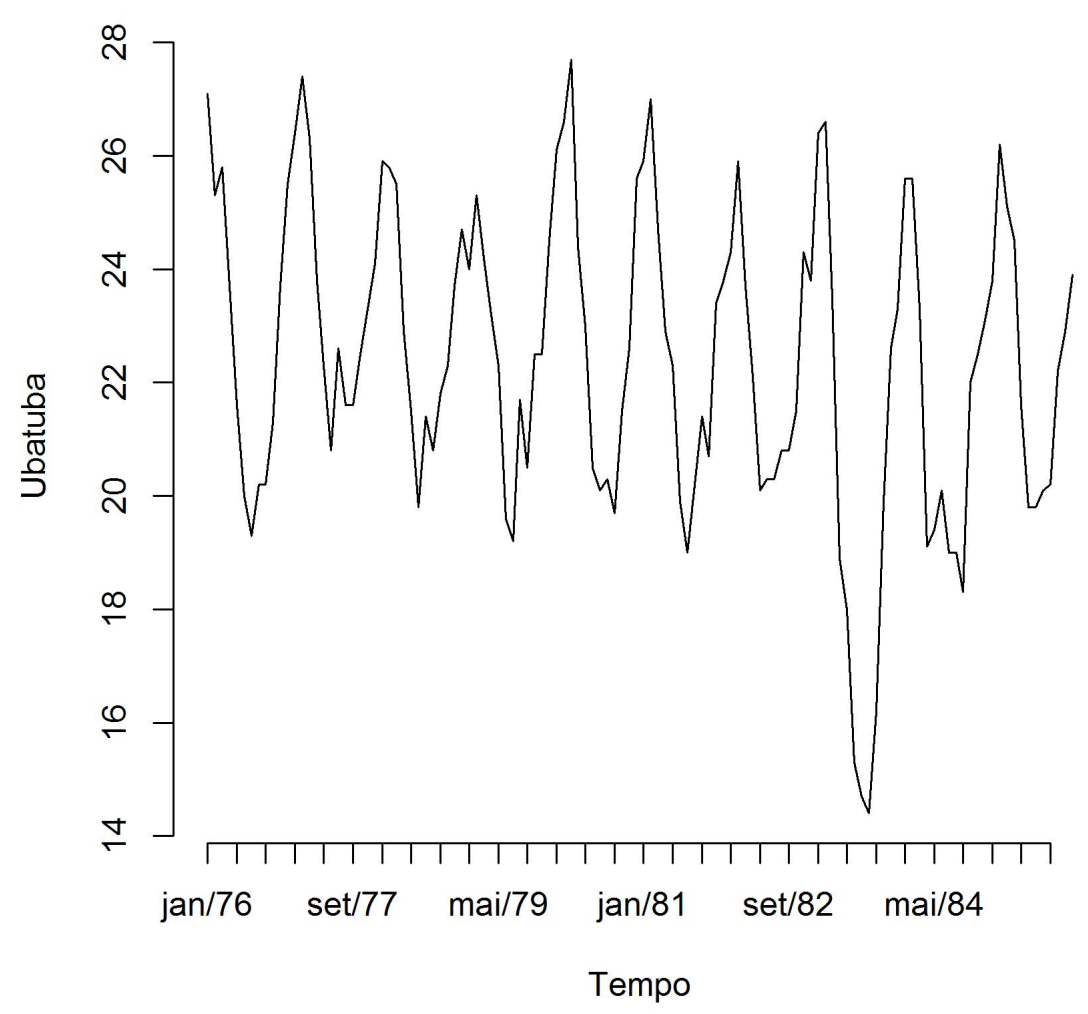

Figura 5.2: Temperatura média mensal de Ubatuba ao longo do tempo.

A Figura 5.2 nos mostra como a temperatura média de Ubatuba se comporta com o decorrer do tempo. Com este gráfico, podemos perceber que controlar o efeito do tempo parece ser importante para termos um efeito entre as duas temperaturas sem o viés de tempo, visto que conseguimos perceber claramente uma série periódica.

Após esta breve etapa de análise descritiva, prosseguiremos para tentar explicar a temperatura média mensal de Ubatuba através da temperatura média mensal de Cananéia.

\subsection{Modelo linear com erros autoregressivos}

Inicialmente, propomos o seguinte modelo para os dados: 


$$
\begin{aligned}
& y_{i}=\mathbf{x}_{i}^{\top} \boldsymbol{\beta}+\epsilon_{i} \quad \mathrm{e} \\
& \epsilon_{i}=\rho \epsilon_{i-1}+e_{i}, \quad-1<\rho<1,
\end{aligned}
$$

em que $\epsilon_{0}=0$.

Este modelo é o mesmo que foi apresentado na Seção 2.3. Cada vetor $\mathbf{x}_{i}$ é composto por um valor igual a 1 , representado o intercepto, e pela $i$-ésima temperatura de Cananéia, enquanto $y_{i}$ representa a $i$-ésima temperatura de Ubatuba. Nesta primeira tentativa, estamos supondo que o erro $e_{i}$ é proveniente de uma distribuição normal de média 0 e variância igual a $\sigma^{2}$.

O ajuste deste modelo foi realizado através do procedimento de CochraneOrcutt, descrito na Seção 2.3, construído no software R.

Os parâmetros estimados desta primeira abordagem estão presentes no Apêndice E, Tabela E.1. Temos que o parâmetro estimado entre a relação entre as duas temperaturas é igual a 0,801, o que nos mostra que, aparentemente, um aumento de 1 grau na temperatura de Cananéia levará a temperatura de Ubatuba a ser elevada em 0,801 graus.

Este ajuste utilizou 4 graus de liberdade e obteve um valor de AIC igual a 292,3 .

Realizaremos, agora, uma análise dos resíduos do modelo.

Nas Figuras 5.3 e 5.4 apresentamos o histograma dos resíduos padronizados e a série desses resíduos ao longo do tempo.

A partir da análise desses gráficos, aparentemente, temos que duas suposições não são válidas. Pelo histograma, temos que a normalidade dos resíduos não parece adequada, pois a distribuição parece possuir uma cauda mais pesada, principalmente à esquerda. Já analisando os resíduos ao longo dos anos, percebemos que parece existir um efeito temporal no ajuste, visto que os resíduos parecem não apresentar média condicional igual a 0 ao longo do tempo.

Também verificamos se toda a autocorrelação serial nos resíduos foi removida. Para isso, mostramos os gráficos das funções de autocorrelação e autocorrelação parcial dos resíduos, que são comumente utilizados em análise de séries temporais. Esses gráficos podem ser vistos na Figuras 5.5.

As funções de autocorrelação e autocorrelação parcial nos fornecem a informação que a série de resíduos padronizados não parece ser uma série de ruído branco, já que estas funções estão acima do valor máximo admitido, linha tracejada, para serem considerados iguais a 0 em alguns lags. Logo, mesmo a inclusão de erros autoregressivos de ordem 1 não foi capaz de remover toda a 


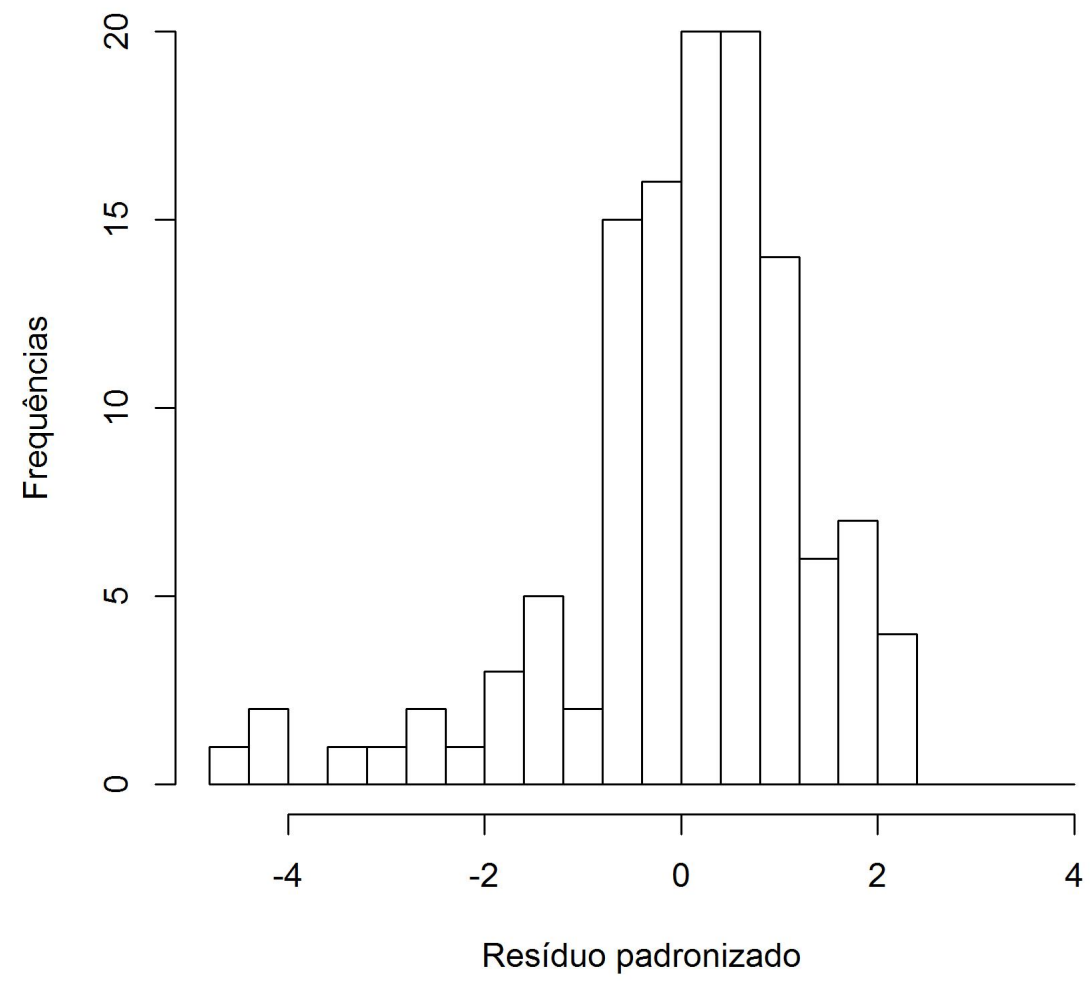

Figura 5.3: Histograma dos resíduos padronizados referente ao ajuste do modelo linear normal com erros autoregressivos aos dados de temperaturas. 


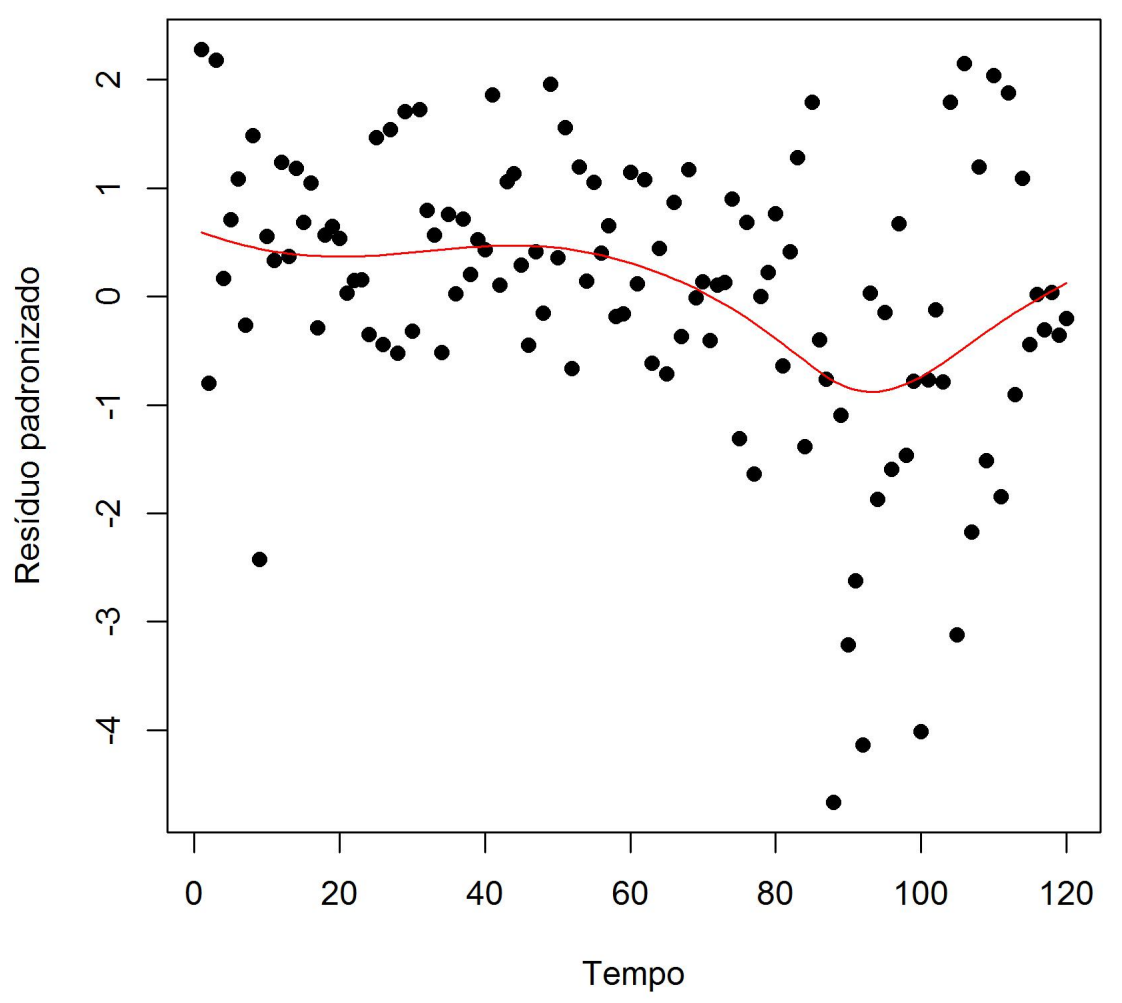

Figura 5.4: Resíduo padronizado ao longo do tempo referente ao ajuste do modelo linear com erros autoregressivos aos dados de temperaturas, com um spline estimado aos dados em vermelho. 
Função de autocorrelação dos resíduos padronizados

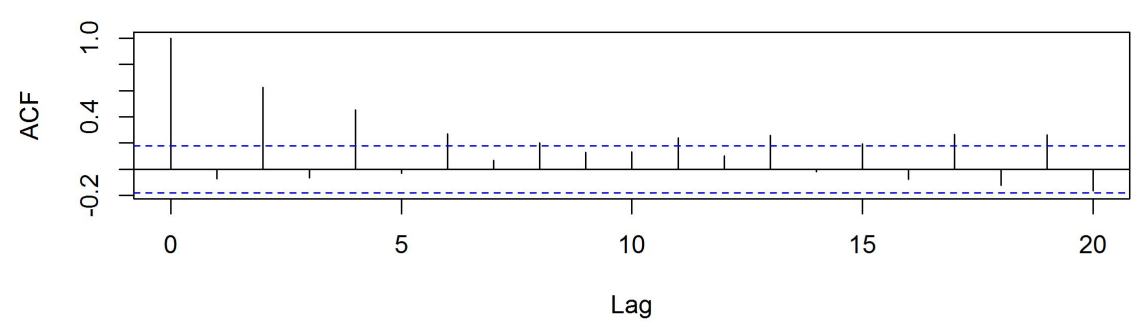

Função de autocorrelação parcial dos resíduos padronizados

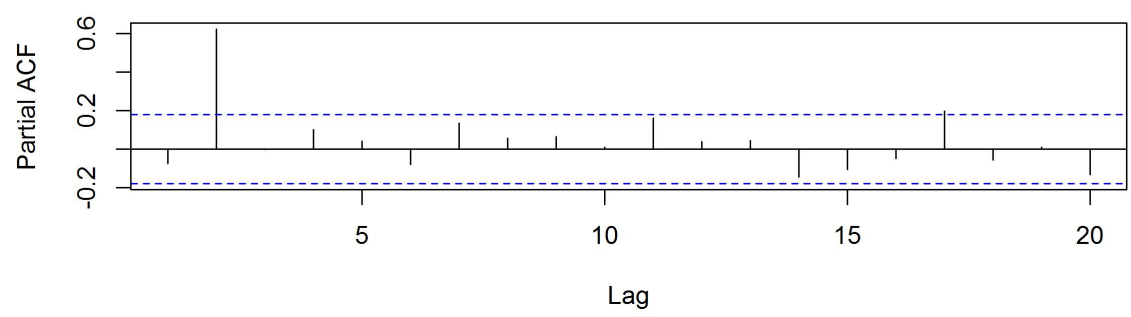

Figura 5.5: Funções de autocorrelações para os resíduos padronizados referente ao ajuste do modelo linear com erros autoregressivos aos dados de temperaturas. 
autocorrelação, sendo necessária uma estrutura mais complexa de autocorrelação.

Como o ajuste apresentado nesta subseção não eliminou toda a autocorrelação presente nos resíduos e como o efeito do tempo não parece ter sido controlado, tentaremos incluir o efeito do tempo de modo não paramétrico utilizando o modelo estudado neste trabalho. Controlar o efeito do tempo pode nos ajudar a eliminar a necessidade de uma estrutura mais complexa de autocorrelação.

Mesmo com os indícios presentes no ajuste desenvolvido nesta subseção, em que os erros não parecem ser provenientes de uma distribuição normal, iremos, primeiramente, realizar o ajuste supondo normalidade, sendo apresentado na Seção 5.3.

\subsection{Modelo parcialmente linear normal AR(1)}

Nesta seção, consideramos novamente o modelo ajustado no Capítulo 1, ou seja, temos

$$
y_{i}=\mathbf{x}_{i}^{\top} \beta+f\left(t_{i}\right)+\epsilon_{i},
$$

em que $\mathbf{x}_{i}$ representa um vetor com um valor igual a 1 e com a $i$-ésima temperatura média de Cananéia, $y_{i}$ a temperatura média de Ubatuba, $f\left(t_{i}\right)$ uma função do tempo para controlar possíveis efeitos do tempo, $\epsilon_{i}$ são erros aleatórios tais que $\epsilon_{1}=e_{1}$ e $\epsilon_{i}=\rho \epsilon_{i-1}+e_{i}$, para $i=2, \ldots, n$, com $e_{i}$ independentes e identicamente distribuídos $N\left(0, \sigma^{2}\right)$.

A estimação, assim como no Capítulo 1, foi feita por meio do método iterativo descrito no Capítulo 3 baseado no algoritmo backfitting. Todo o processo foi realizado no software $R$.

Conforme discutimos durante a explicação do processo iterativo, o parâmetro de suavização $(\alpha)$ tem um importante papel no ajuste do modelo. Para escolhermos o valor de $\alpha$, optamos por analisar conjuntamente o valor de AIC, o número de graus de liberdade efetivemente utilizados no ajuste, o valor de AIC corrigido e o critério de verossimilhança penalizado. Na Figura 5.6, apresentamos o AIC e os graus de liberdade para diferentes valores de $\alpha$.

Podemos notar que à medida em que o parâmetro de suavização aumenta, o número efetivo de graus de liberdade diminui, o que já era esperado. Já analisando a medida de AIC, observamos que ocorre o inverso, resultando em um modelo com um valor melhor no critério de Akaike à medida em que diminuímos o valor de $\alpha$. No entanto, o preço a se pagar por esse melhor valor 

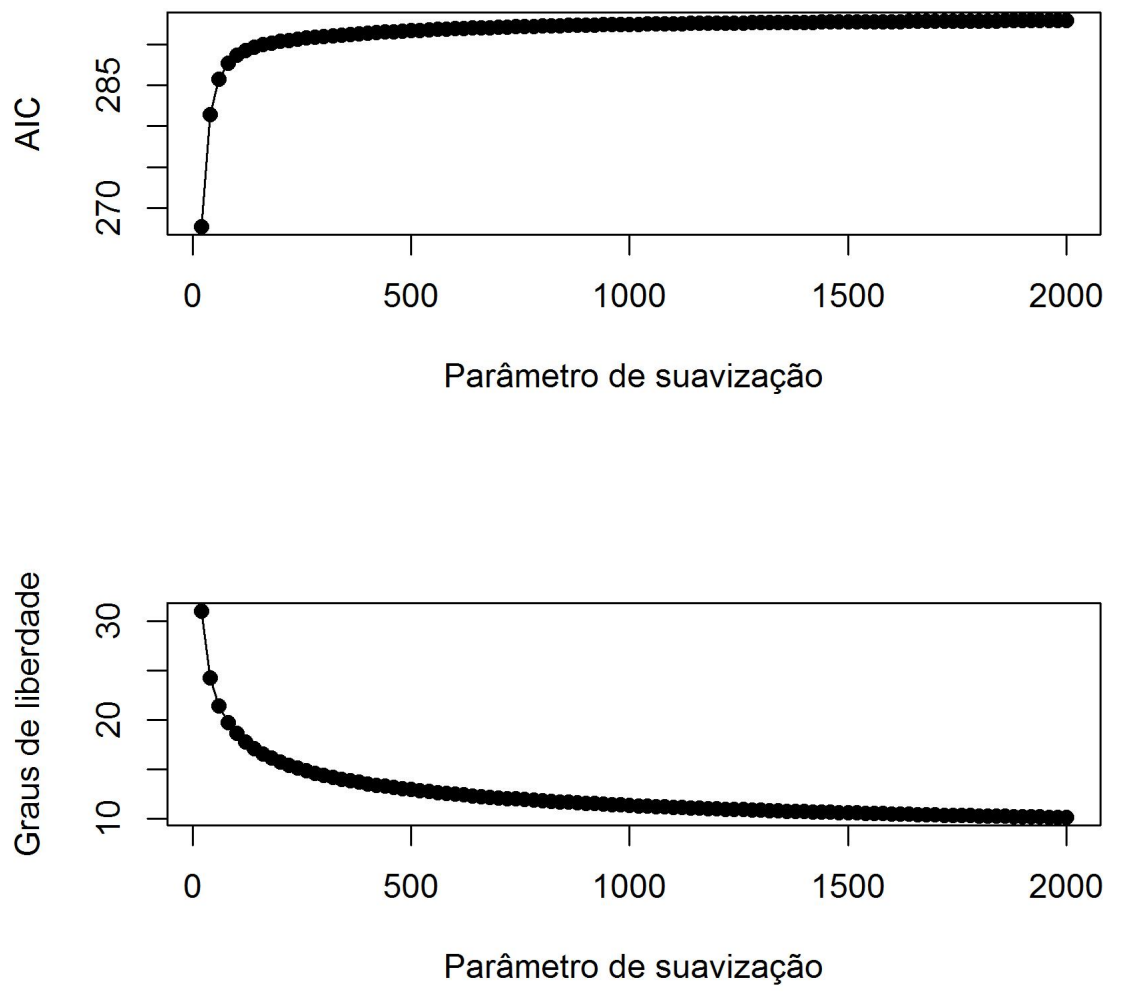

Figura 5.6: O primeiro gráfico mostra o valor de AIC para diferentes valores de $\alpha$, enquanto que o segundo mostra o número de graus de liberdade para cada um desses ajustes, considerando o modelo parcialmente linear normal com erros autoregressivos $A R(1)$. 


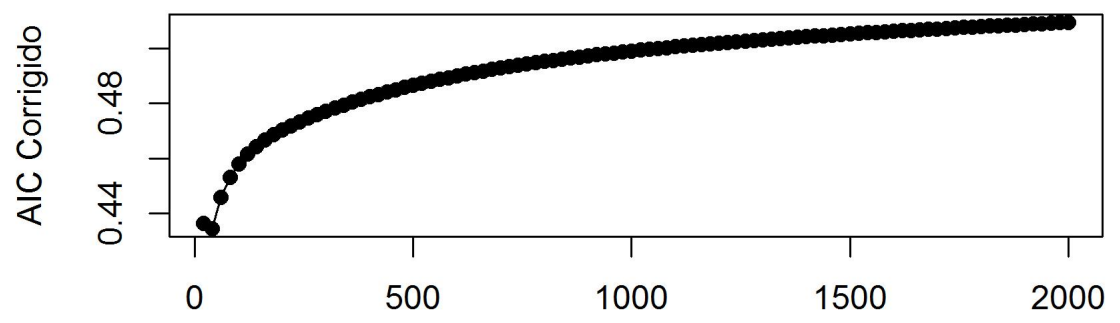

Parâmetro de suavização

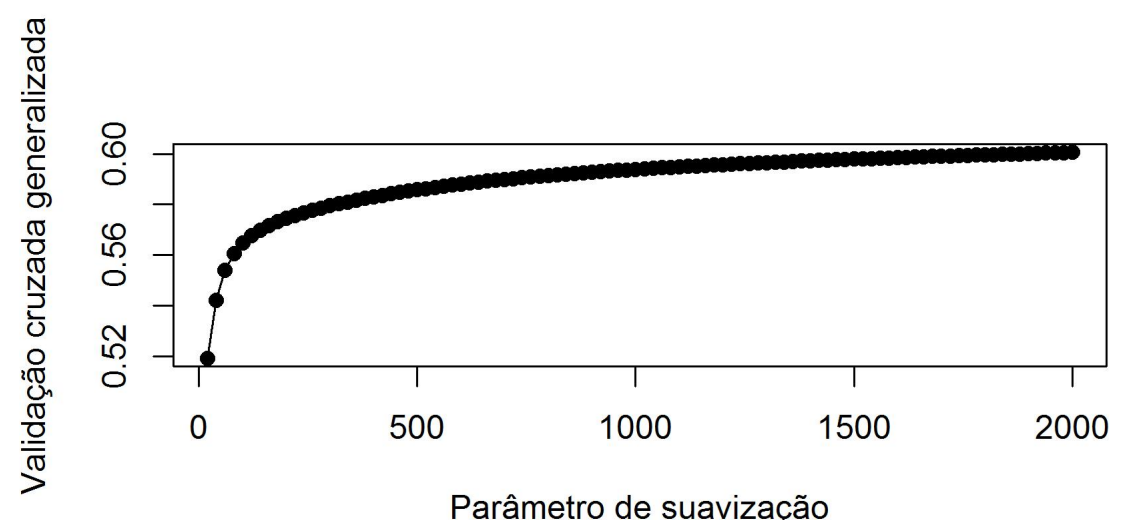

Parâmetro de suavização

Figura 5.7: O primeiro gráfico mostra o valor do AIC corrigod para diferentes valores de $\alpha$, enquanto que o segundo mostra o critério de validação cruzada generalizada para cada um desses ajustes, considerando o modelo parcialmente linear normal com erros autoregressivos $A R(1)$. 
de AIC é alto, visto que o número de graus de liberdade é muito alto. Portanto, a fim de termos um modelo parcimonioso, decidimos escolher um valor de $\alpha$ alto, pois o número de graus de liberdade é relativamente mais baixo e não perdemos muito em relação ao AIC. Assim, utilizamos o valor de 2000 para o parâmetro de suavização, pois parece ser um ponto em que o número de graus de liberdade e o valor de AIC se estabilizam.

Para confirmar a escolha deste valor, construímos o mesmo gráfico com o AIC corigido e o critério de validação cruzada generalizada para diferentes valores de $\alpha$, apresentado na Figura 5.7. Podemos ver por meio destes gráficos, que a escolha do valor de 2000 realmente parece ser uma escolha razoável com um baixo número de graus de liberdade utilizados.

Os valores estimados dos parâmetros do modelo, utilizando $\alpha$ igual a 2000, podem ser vistos no Capítulo 1 ou na Tabela E.2 no Apêndice E. Não apresentamos as estimativas referentes à parte não paramétrica devido à grande quantidade de estimativas (120). Este ajuste apresentou valor de AIC igual a 292, 93 e utilizou 10, 14 graus de liberdade.

Para este ajuste, também construímos a mesma análise de diagnóstico apresentada para o caso anterior. Abaixo, nas Figuras 5.8 e 5.9, apresentamos o histograma dos resíduos padronizados e o gráfico com esses resíduos ao longo do tempo.

Nota-se que os resíduos ainda apresentam alguns valores elevados em valor absoluto, principalmente valores negativos, evidenciando caudas mais pesadas. Talvez o uso de uma distribuição assimétrica seja mais apropriado para esse conjunto de dados. Já analisando os resíduos padronizados ao longo do tempo, notamos que o spline estimado para os resíduos é aproximadamente uma reta em torno do valor 0. Assim, com este ajuste, a média dos resíduos condicional ao tempo parece ser igual 0 , fornecendo indícios que o efeito do tempo foi controlado.

Apesar de não apresentarmos todas as estimativas da parte não paramétrica do ajuste, apresentamos essas estimativas, ao longo do tempo, na Figura 5.10. Comparando o efeito estimado do tempo com a Figura 5.4, podemos observar que o referido efeito é muito similar à curva estimada dos resíduos padronizados, ao longo do tempo, do ajuste anterior. Portanto, a inclusão da função não paramétrica do efeito do tempo está se ajustando aos resíduos obtidos sem controlar por este efeito. Esta ilustração ajuda a compreender como a função não paramétrica do tempo está contribuindo no ajuste.

As Figuras 5.8 e 5.9 levantam duas suspeitas, de que a distribuição do erro não é proveniente de uma distribuição normal e que os resíduos não apresentam 


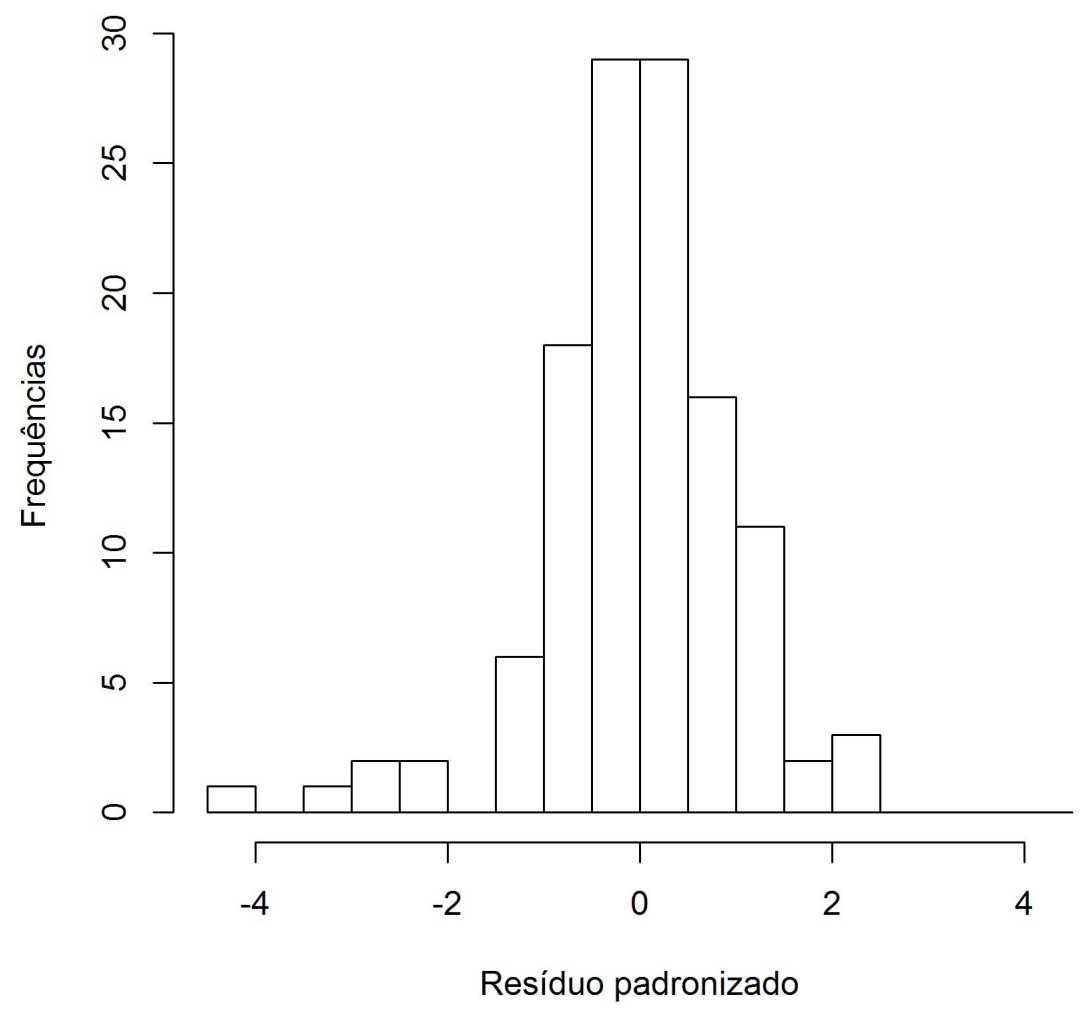

Figura 5.8: Histograma dos resíduos padronizados para os dados de temperatura considerando o modelo parcialmente linear normal com erros autoregressivos $A R(1)$. 


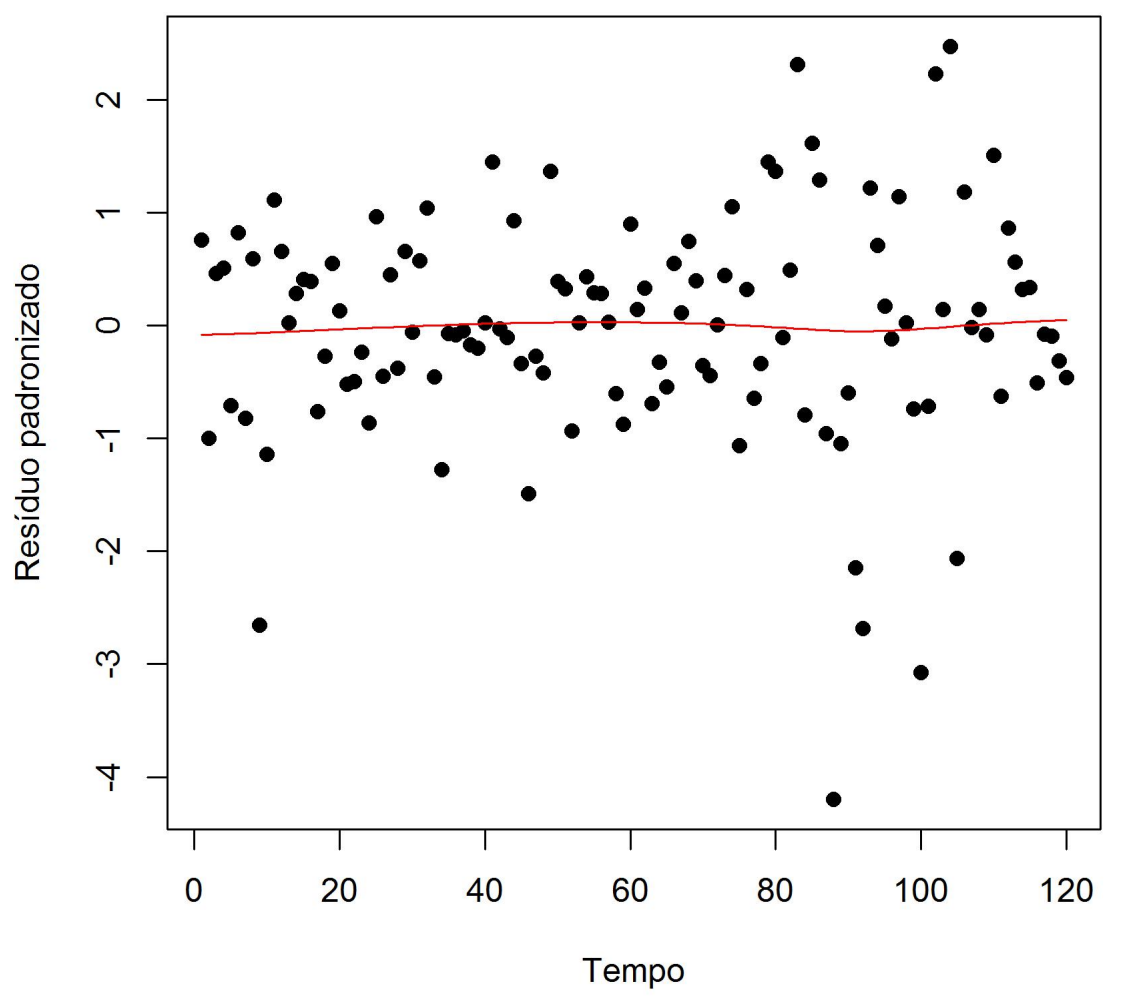

Figura 5.9: Residuos padronizados ao longo do tempo para os dados de temperatura considerando o modelo parcialmente linear normal com erros autoregressivos AR(1), com um spline estimado aos dados em vermelho. 


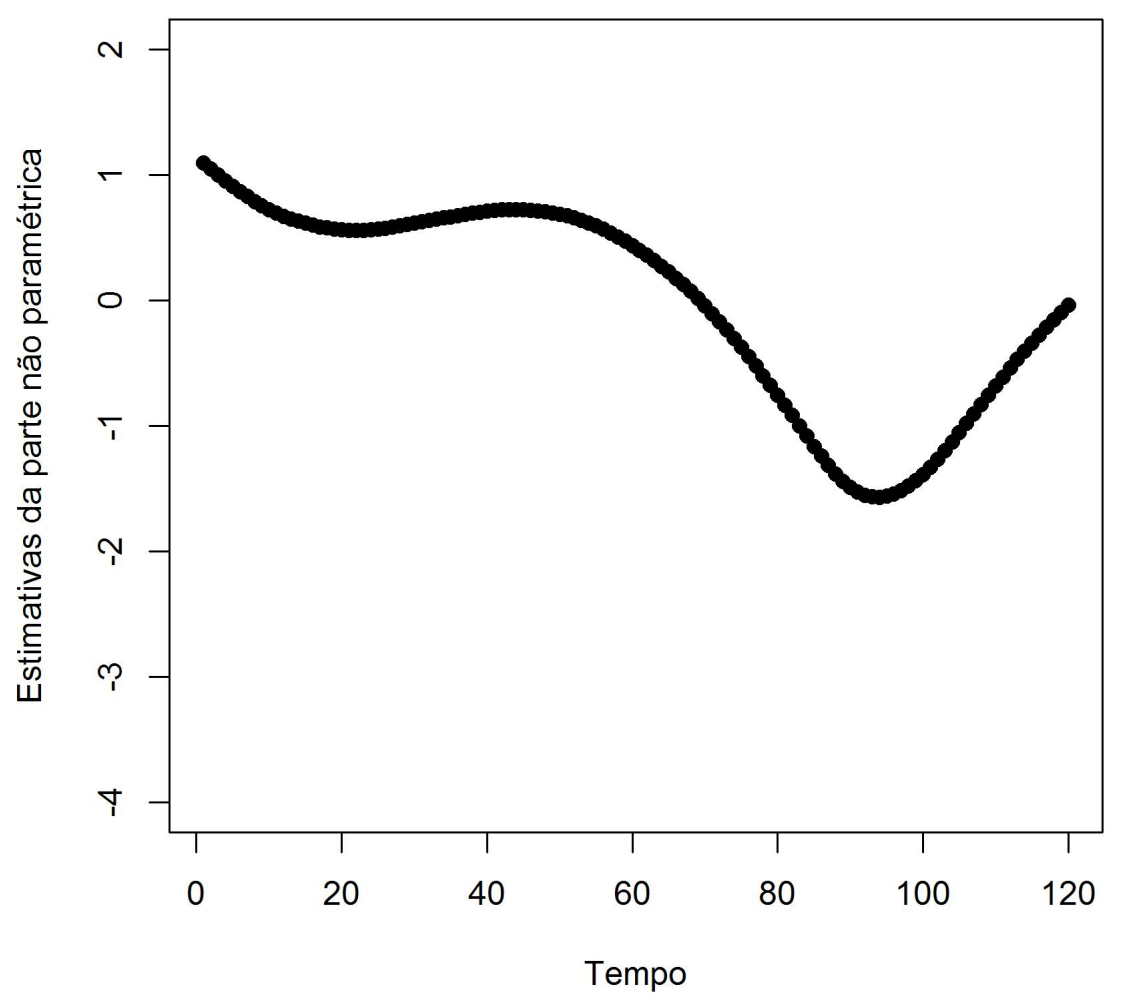

Figura 5.10: Estimativas do efeito do tempo para os dados de temperatura considerando o modelo parcialmente linear normal com erros autoregressivos $A R(1)$. 
mais autocorrelação serial. Para confirmar ou não tais suspeitas, primeiramente construímos o gráfico 5.11 que representa o gráfico normal de probabilidades com banda de confiança simulada, conhecida como envelope. Já na Figura 5.12, mostramos as funções de autocorrelação e autocorrelação parcial dos resíduos padronizados.

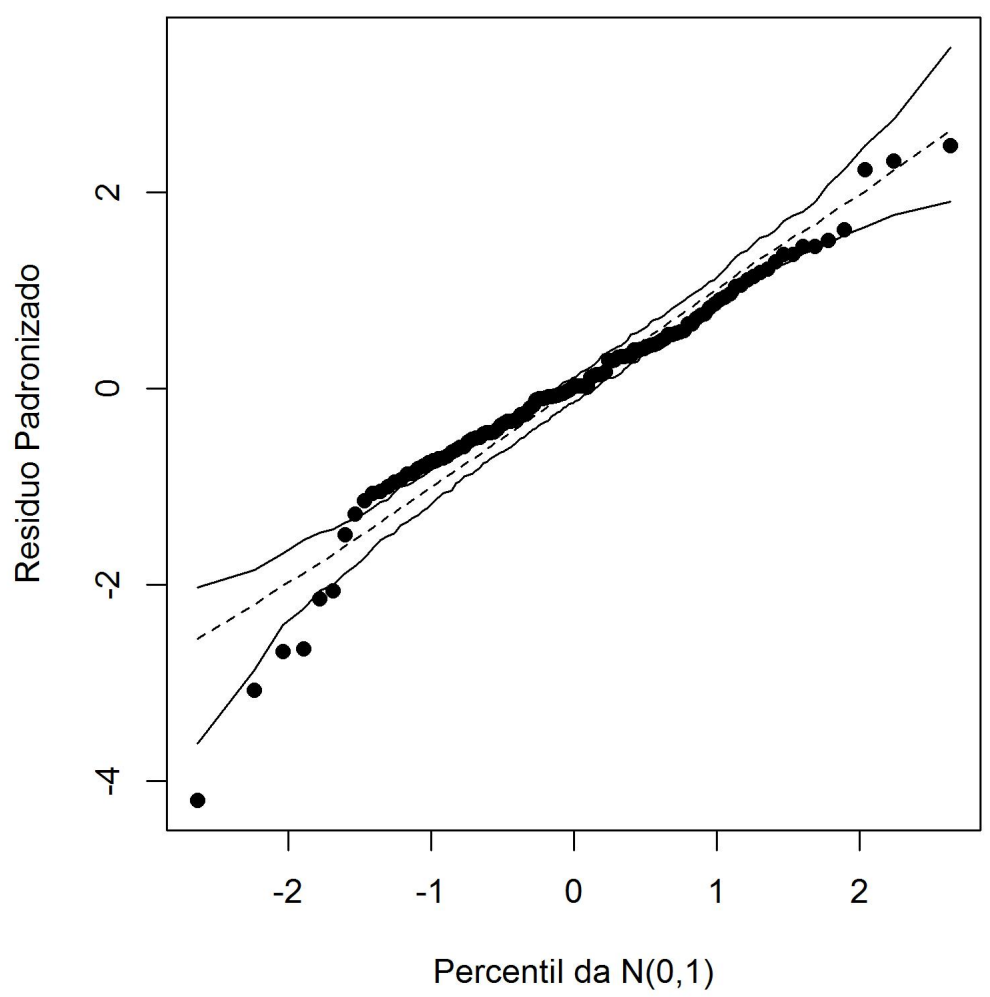

Figura 5.11: Gráfico normal de probabilidades para os resíduos padronizados referente ao modelo parcialmente linear normal com erros autoregressivos AR(1) ajustado aos dados de temperaturas.

Essas figuras reforçam nossos levantamentos iniciais. Podemos notar pelos gráficos de autocorrelação e autocorrelação parcial que os resíduos não apresentam mais autocorrelação visto que em praticamente todos os lags analisados, essas funções são menores em valores absoluto do que a linha tracejada, limite de não rejeição de autocorrelação igual a 0. Para confirmarmos esta hipótese, 
Função de autocorrelação dos resíduos padronizados

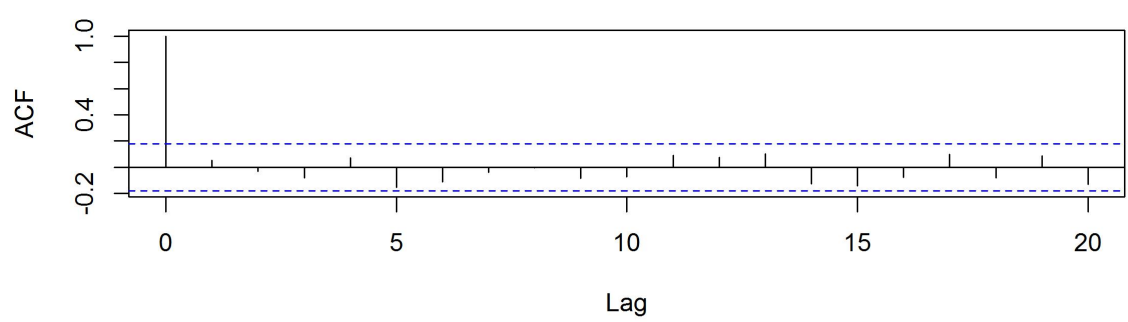

Função de autocorrelação parcial dos resíduos padronizados

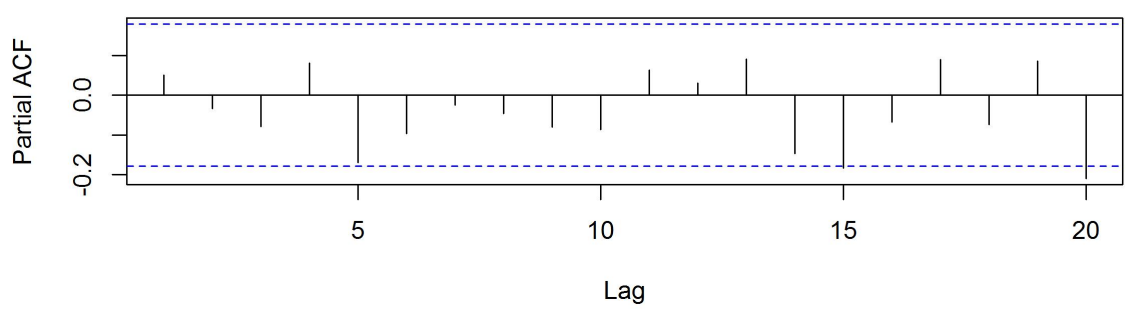

Figura 5.12: Funções de autocorrelação e autocorrelação parcial para os resíduos padronizados referente ao modelo parcialmente linear normal com erros autoregressivos AR(1) ajustado aos dados de temperaturas. 
realizamos o teste de Durbin-Watson, que apresentou estatística $d$ igual a 1,89 e um respectivo valor-p de 0,24 , isto é, consideramos que a série não apresenta autocorrelação. Portanto, aparentemente conseguimos eliminar a presença de autocorrelação nos resíduos.

Pelo gráfico normal de probabilidades com a banda de confiança, conforme salientamos no Capítulo 1, os resíduos parecem ter uma distribuição com caudas mais pesadas do que a distribuição normal, visto que alguns pontos estão fora da banda de confiança de $95 \%$.

Com o ajuste presente nesta seção conseguimos melhorias em relação a aceitação de algumas suposições. A principal suposição ainda não alcançada é a de normalidade dos erros. Portanto, na próxima seção, ajustaremos esse mesmo modelo, porém, iremos supor que a distribuição dos erros é uma t de Student.

\subsection{Modelos parcialmente lineares t de Student $\operatorname{AR}(1)$}

Dos pontos comentados até o momento, fica claro que o modelo aqui desenvolvido já parece ser uma melhor escolha de modelagem do que as utilizadas anteriormente. Nesta seção, iremos alterar a suposição de normalidade para a suposição t de Student para tentar acomodar a suposição de distribuição dos erros. Temos novamente que o modelo é dado da mesma forma, isto é,

$$
\begin{aligned}
y_{i} & =\mathbf{x}_{i}^{\top} \boldsymbol{\beta}+f\left(t_{i}\right)+\epsilon_{i} \\
\epsilon_{i} & =\rho \epsilon_{i-1}+e_{i},
\end{aligned}
$$

no entanto, agora $e_{i}$ é proveniente de uma distribuição t de Student com $v$ graus de liberdade.

Como no caso normal, devemos escolher o valor do parâmetro de suavização $\alpha$. Além de $\alpha$, o número de graus de liberdade também deve ser escolhido. Poderíamos estimar esse parâmetro por meio do procedimento de máxima verossimilhança, no entanto, segundo Lucas [1997] é aconselhável fixar a priori o número de graus de liberdade.

Assim, realizamos o mesmo procedimento contruído para o caso normal para diferentes valores de $v$. Com isso, podemos escolher conjuntamente os valores de $\alpha$ e de $v$. Na Figura 5.13, apresentamos o valor de AIC para vários 
combinações desses parâmetros.
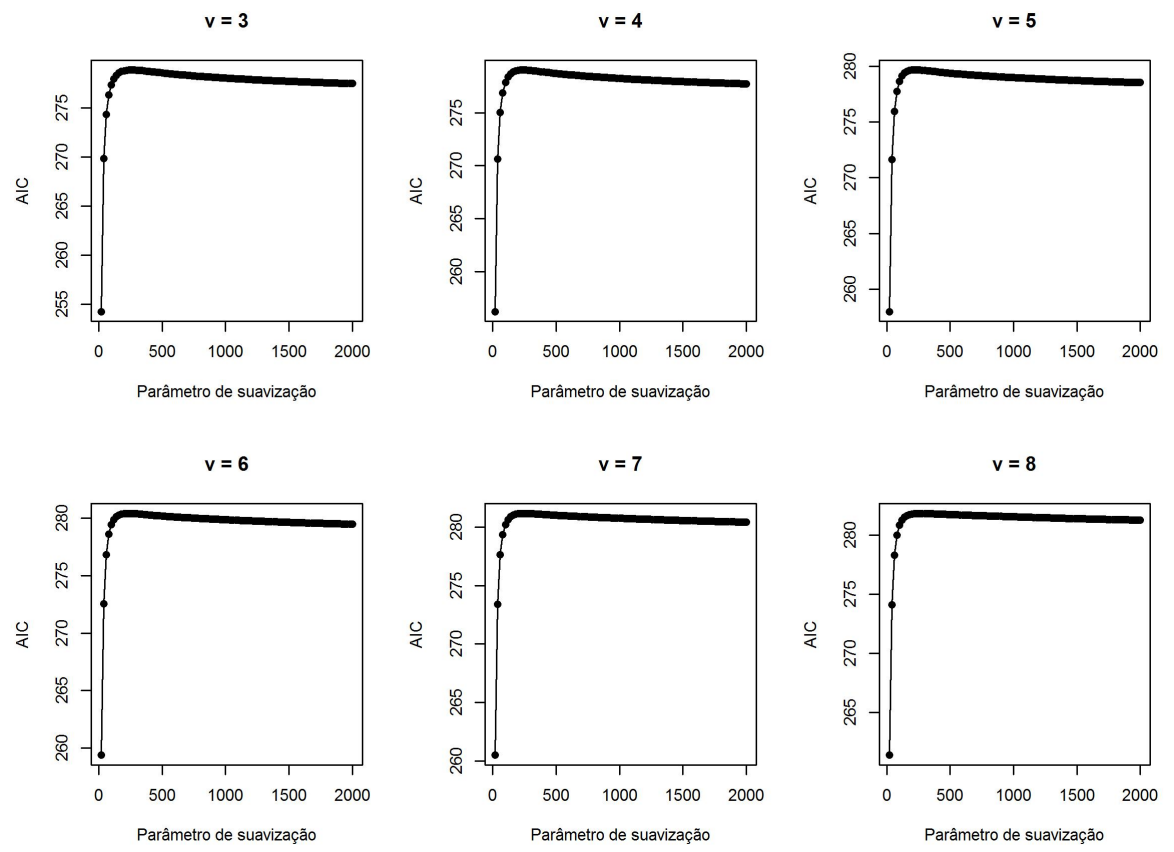

Figura 5.13: Os gráficos mostram o valor de AIC para diferentes valores de $\alpha$ $e$ de $v$ (graus de liberdade da $t$ de Student) considerando o modelo parcialmente linear $t$ de Student com erros autoregressivos AR(1).

Podemos observar que à medida em que aumentamos o número de graus de liberdade da distribuição do erro, o ajuste parece apresentar pioras em relação ao critério de Akaike. Considerar 3 ou 4 graus de liberdade parece produzir melhores resultados sendo praticamente similares. Assim, optamos por continuar o ajuste considerando 3 graus de liberdade.

Com essa escolha, mostramos na Figura 5.14 novamente os valores de AIC e o número de graus de liberdade efetivos para diferentes valores de $\alpha$, a fim de analisar com mais cuidado essa funções em relação ao parâmetro de suavização. Podemos verificar que à medida em que aumentamos o valor de $\alpha$, melhoramos a medida de AIC e diminuímos os graus de liberdade efetivos. No entanto, notamos para $\alpha$ igual a 2000 que as curvas estão praticamente estáveis. Assim, novamente, escolhemos o valor de 2000 para o parâmetro de suavização. Logo, ainda temos a vantagem de podermos comparar, de forma mais igualitária, este ajuste com o caso anterior. 
Assim como no caso normal, também construímos os outros dois critérios, o AIC corrigido e a validação cruzada generalizada, apresentados na Figura 5.15. Estes gráficos nos mostram que essas medidas penalizam mais o número de graus de liberdade utilizados, indicando melhores ajustes para altos valores de $\alpha$, ao contrário do uso de apenas do critério de Akaike. No entanto, notamos que as duas medidas parecem convergirem depois de $\alpha$ maior do que 500, o que nos mostra que a escolha do valor 2000 é apropriada.
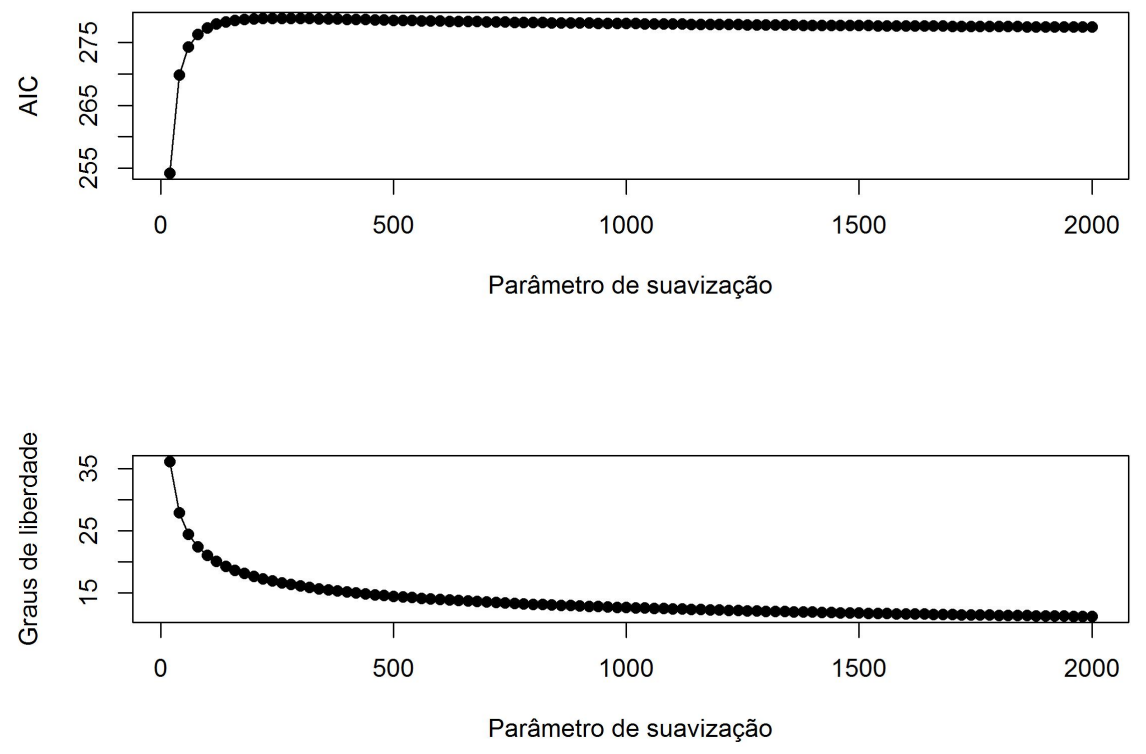

Figura 5.14: O primeiro gráfico mostra o valor de AIC para diferentes valores de $\alpha$, enquanto que o segundo mostra o número de graus de liberdade efetivo para cada um desses ajustes, considerando o modelo parcialmente linear $t$ de Student com 3 graus de liberdade com erros autoregressivos $A R(1)$.

Os valores estimados do modelo, utilizando $\alpha$ igual a 2000 e 3 graus de liberdade, estão na Tabela E.3, no Apêndice E. O método iterativo utilizado foi o mesmo comentado no Capítulo 3 por meio do software R. Este ajuste apresentou valor de AIC igual a 277,48 e utilizou cerca de 11,20 graus de liberdade. Nota-se que este ajuste apresenta o melhor desempenho ao analisarmos o critério de Akaike dos três modelos apresentados. Além disso, comparando com o ajuste apresentado na Seção 5.3, podemos ver que não há uma grande diferença no número de graus de liberdade utilizados. 


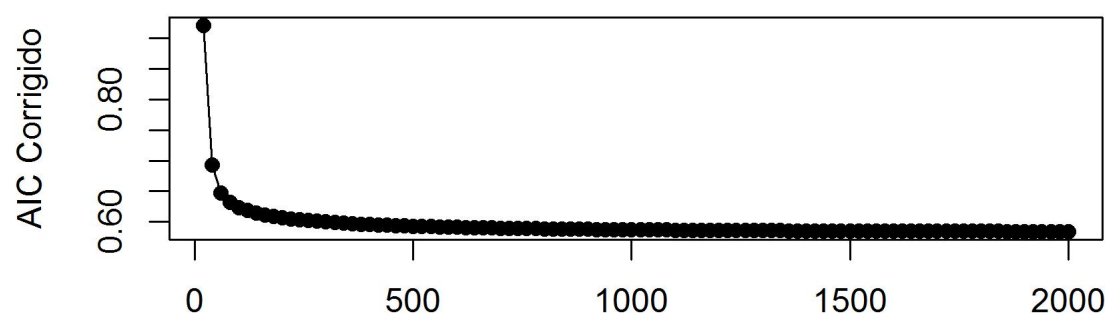

Parâmetro de suavização

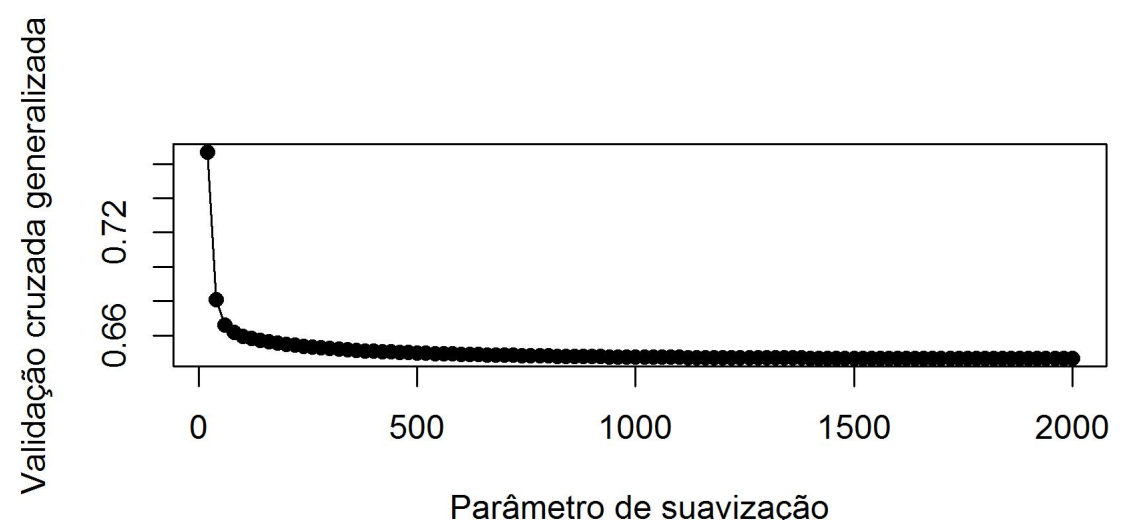

Figura 5.15: O primeiro gráfico mostra o valor do AIC corrigod para diferentes valores de $\alpha$, enquanto que o segundo mostra o critério de validação cruzada generalizada para cada um desses ajustes, considerando o modelo parcialmente linear $t$ de Student com 3 graus de liberdade com erros autoregressivos AR(1). 
Mais uma vez, construímos os mesmos gráficos para estudar a adequação do modelo. Inicialmente, apresentamos o histograma dos resíduos padronizados, além de mostrar esses valores ao longo do tempo, vistos nas Figuras $5.16 \mathrm{e}$ 5.17 .

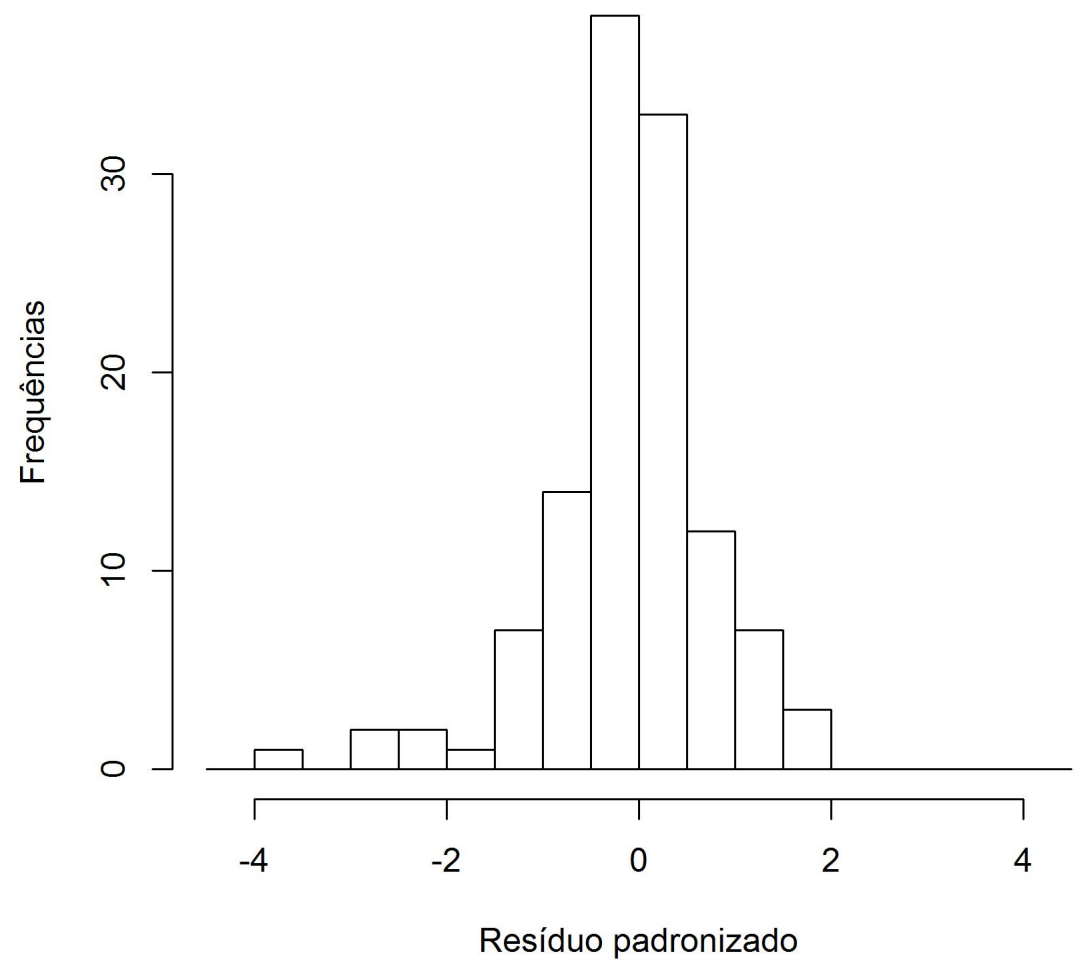

Figura 5.16: Histograma dos resíduos padronizados para os dados de temperatura considerando o modelo parcialmente linear $t$ de Student com erros autoregressivos $A R(1)$.

Podemos notar que a distribuição dos resíduos para este ajuste é muito similar ao ajuste da Seção 5.3. O mesmo pode-se dizer analisando os resíduos ao longo do tempo, em que aparentemente toda autocorrelação serial foi removida, além de termos o efeito do tempo controlado, visto que os resíduos parecem apresentar média 0 condicional a qualquer tempo. Logo, assim como no ajuste anterior, a função não paramétrica do tempo deve apresentar a mesma forma. 


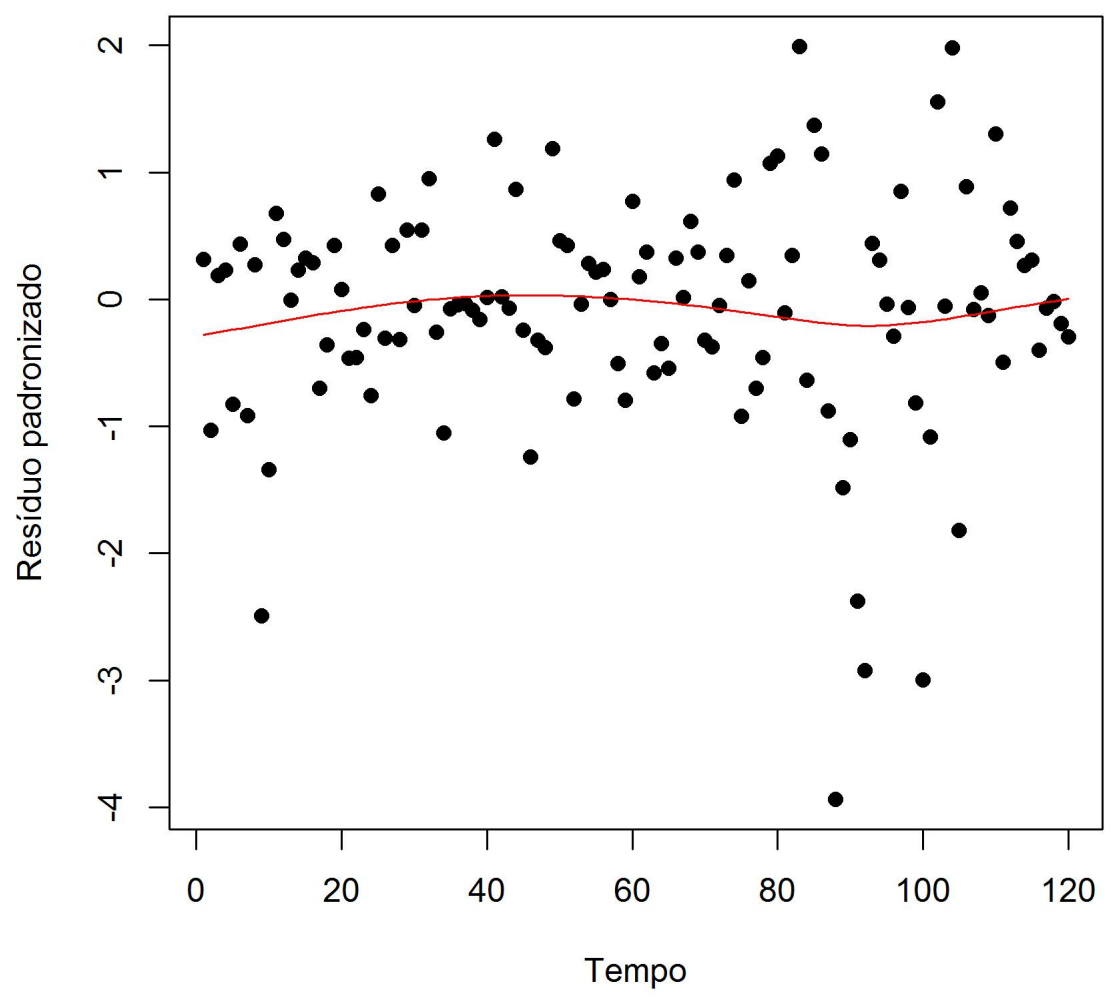

Figura 5.17: Resíduos padronizados ao longo do tempo para os dados de temperatura considerando o modelo parcialmente linear $t$ de Student com erros autoregressivos AR(1), com um spline estimado aos dados em vermelho. 
Para verificarmos se isto realmente ocorre, construímos a função do efeito estimado do tempo, apresentada na Figura 5.18, que nos confirma tal hipótese, isto é, o efeito do tempo é muito similar. Lembrando que não apresentamos as estimativas da parte não paramétrica pelo mesmo motivo anterior, a grande quantidade de efeitos estimados.

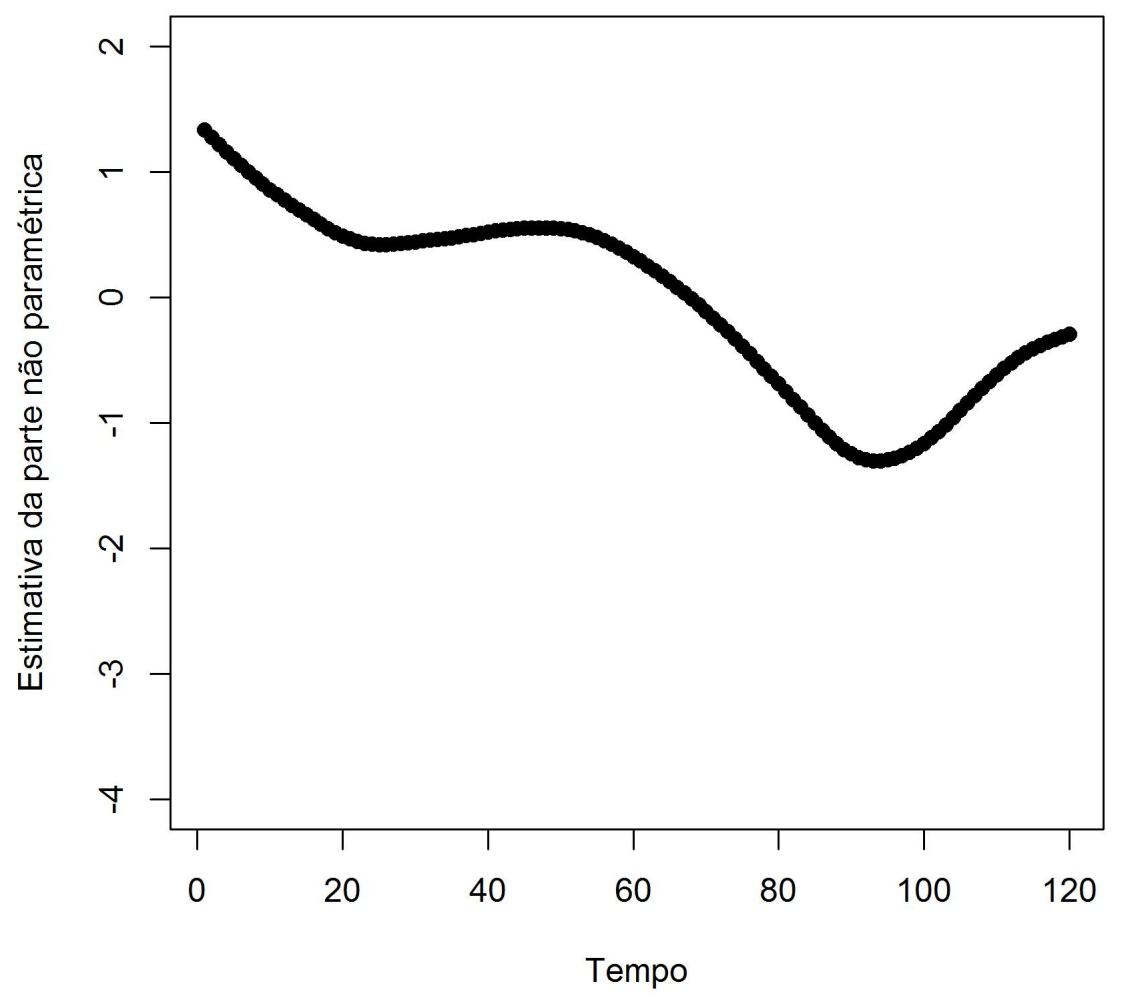

Figura 5.18: Estimativas do efeito do tempo para os dados de temperatura considerando o modelo parcialmente linear $t$ de Student com erros autoregressivos $A R(1)$.

Do mesmo modo que na seção anterior, construímos o gráfico normal de probabilidades com o envelope simulado considerando a distribuição t de Student com 3 graus de liberdade e os gráficos das funções de autocorrelações. Estes gráficos são apresentados respectivamente nas Figuras 5.19 e 5.20. 


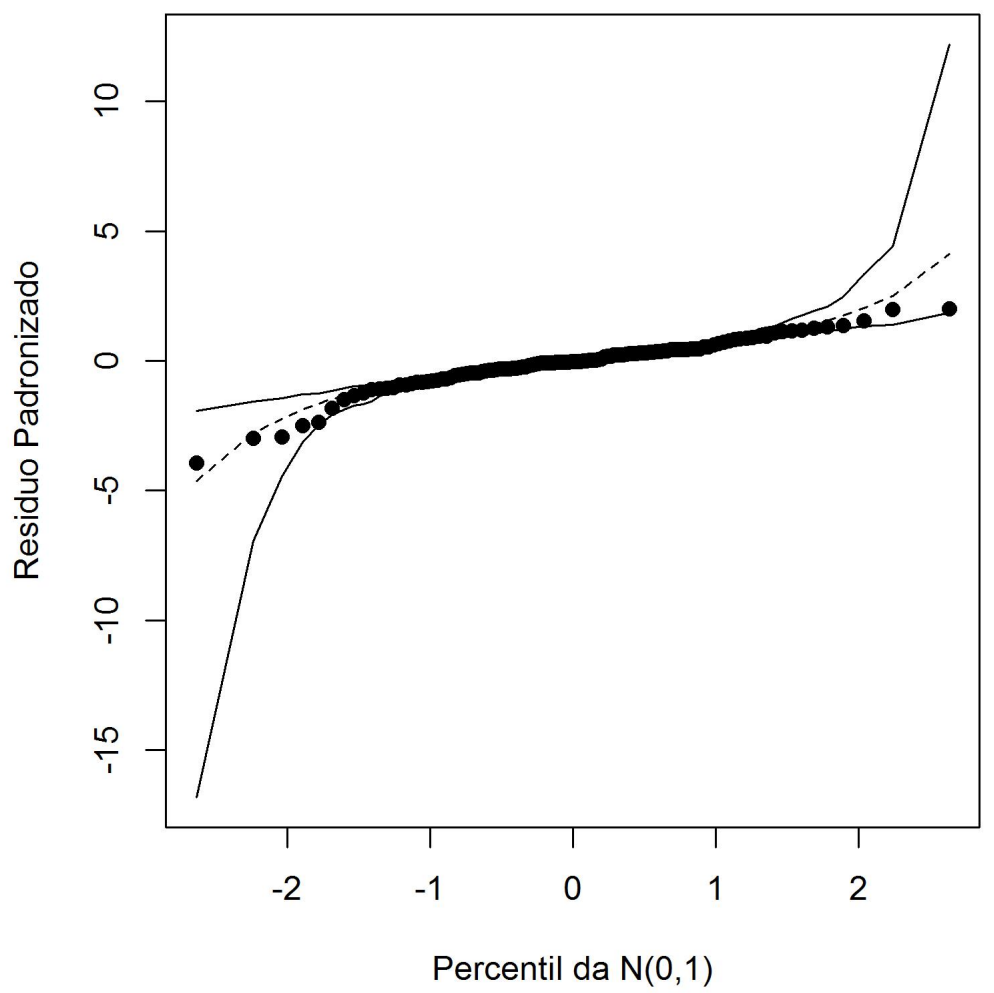

Figura 5.19: Gráfico normal de probabilidades para os resíduos padronizados referente ao modelo parcialmente linear $t$ de Student com erros autoregressivos $A R(1)$ ajustado aos dados de temperaturas. 
Função de autocorrelação dos resíduos padronizados

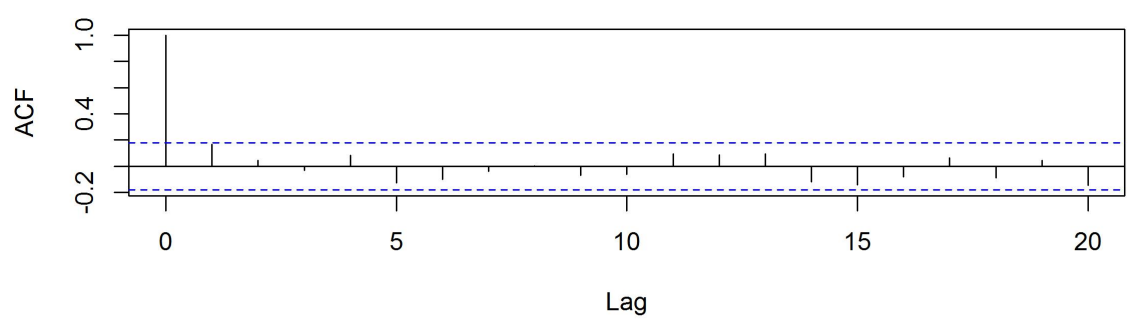

Função de autocorrelação parcial dos resíduos padronizados

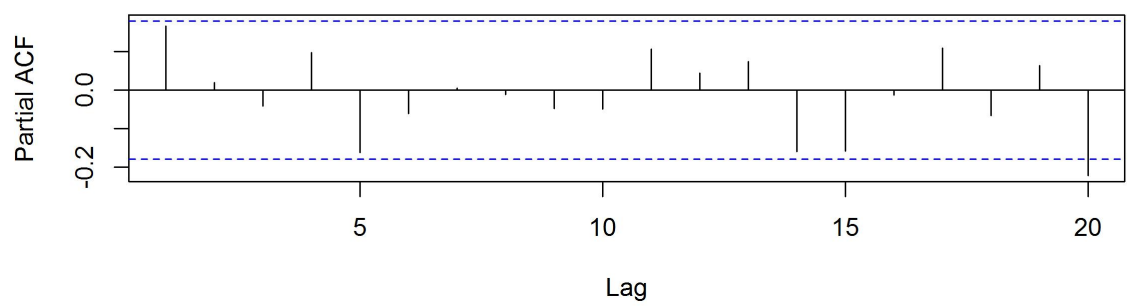

Figura 5.20: Funções de autocorrelação e autocorrelação parcial para os resíduos padronizados referente ao modelo parcialmente linear $t$ de Student com erros autoregressivos AR(1) ajustado aos dados de temperaturas. 
Com o gráfico normal de probabilidade, temos que todos os resíduos padronizados estão dentro das bandas produzidas pelo envelope, não nos fornecendo indícios de que a distribuição dos erros não seja realmente proveniente de uma distribuição t de Student com 3 graus de liberdade. Fica evidente que a distribuição considerada nesta seção se adequa melhor aos dados que a distribuição normal (vide Figura 5.11).

Pelas funções de autocorrelação e autocorrelação parcial, podemos observar que a autocorrelação serial parece ter sido totalmente removida, visto que dos lags 1 até o 20, praticamente todos os pontos das duas funções estão abaixo da linha tracejada que representa o valor máximo para não rejeitarmos a hipótese de que sejam iguais a 0 . Como trata-se de uma série de temperaturas mensais, uma suspeita inicial era a presença, também, de autocorrelação períodica de 12 lags. No entanto, não percebemos este fenômeno analisando os resíduos.

Portanto, o modelo apresentado nesta seção, além de produzir melhores resultados pelo critério de AIC, também parece satisfazer as principais suposições impostas, isto é, o uso de uma distribuição simétrica com caudas mais pesadas é mais adequada para a situação aqui discutida.

Na Figura 5.21, temos a série de temperatura de Ubatuba observada e estimada. Podemos ver que os valores estimados estão muito próximos dos valores observados, evidenciando um bom ajuste do modelo.

Os três modelos ajustados neste capítulo apresentam estimativas diferentes para a parte paramétrica do ajuste. Para visualizar tal fato mais facilmente, construímos a Tabela 5.1 com os intervalos de confiança de $95 \%$ para as estimativas dos parâmetros com os três ajustes, evidenciando as mudanças das estimativas.

Na próxima seção apresentaremos uma análise de diagnóstico mais detalhada para os modelos parcialmente linear com erros autoregressivos normal e t de Student.

Tabela 5.1: Intervalos de confiança de 95\% para as estimativas da parte paramétrica dos três ajustes apresentados referentes aos dados de temperatura. LI - Limite inferior, LS - Limite superior

\begin{tabular}{r|cc|cc|cc}
\hline & \multicolumn{2}{|c}{ Ajuste Seção 5.2 } & \multicolumn{2}{c}{ Ajuste Seção 5.3 } & \multicolumn{2}{c}{ Ajuste Seção 5.4 } \\
\hline & LI & LS & LI & LS & LI & LS \\
\hline Intercepto & 3,08 & 7,02 & 4,5 & 4,79 & 5,06 & 5,28 \\
Cananéia & 0,715 & 0,897 & 0,818 & 0,832 & 0,801 & 0,813 \\
$\sigma^{2}$ & 0,63 & 0,68 & 0,555 & 0,581 & 0,70 & 0,74 \\
& 0,65 & 0,88 & 0,535 & 0,568 & 0,46 & 0,48 \\
\hline
\end{tabular}




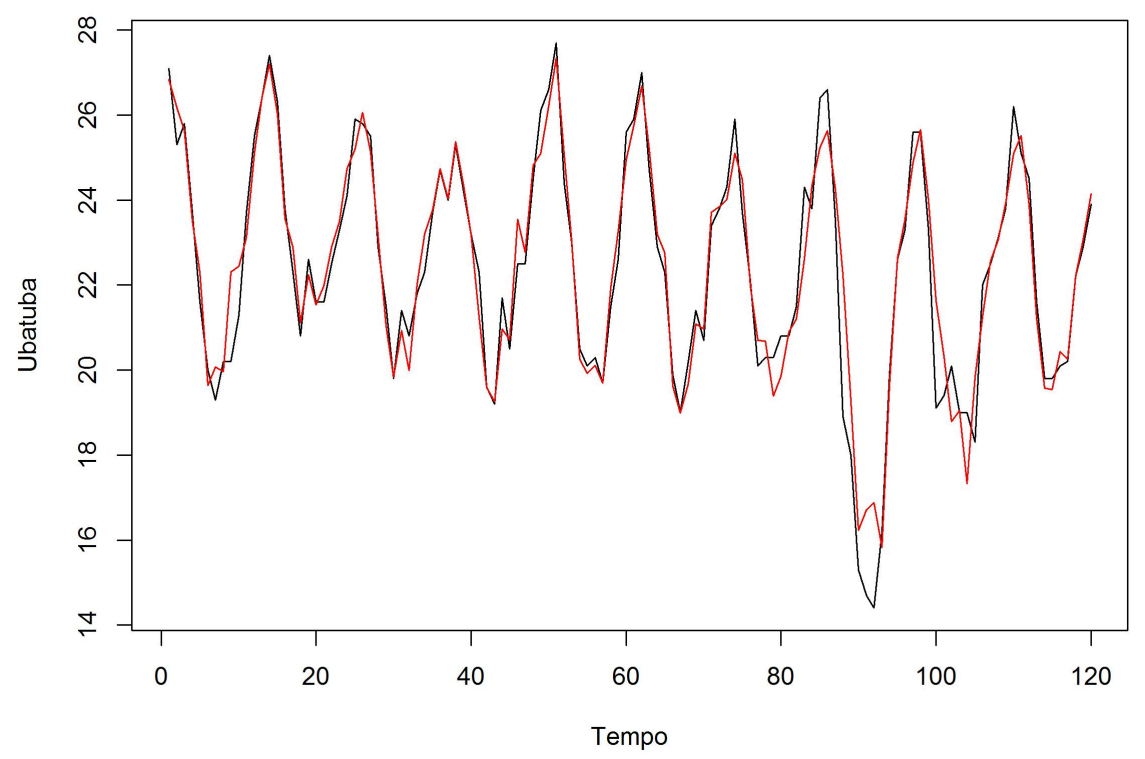

Figura 5.21: Temperatura de Ubatuba observada e estimada (em vermelho pontilhado) referente ao modelo parcialmente linear $t$ de Student com erros autoregressivos $A R(1)$.

\subsection{Influência local}

Com o intuito de realizarmos uma análise de diagnóstico mais detalhada para os ajustes apresentados neste capítulo, construímos os gráficos de influência local sob o esquema de ponderação de casos para os modelos parcialmente lineares com erros autoregressivos de primeira ordem, sob suposição de normalidade e sob a distribuição t de Student.

A Figura 5.22 apresenta esses gráficos para os ajustes supondo erros normais e supondo erros t de Student. Estes gráficos foram construídos para avaliar as estimativas de $\boldsymbol{\theta}$ e não em apenas um subconjunto específico de $\boldsymbol{\theta}$.

Podemos notar que os ajustes com as duas distribuições parecem apresentar algumas observações que possuem uma influência elevada considerando este esquema de perturbação. No entanto, para o caso t de Student, estas observações, aparentemente, são um pouco menos influentes. Analisando esta figura, 

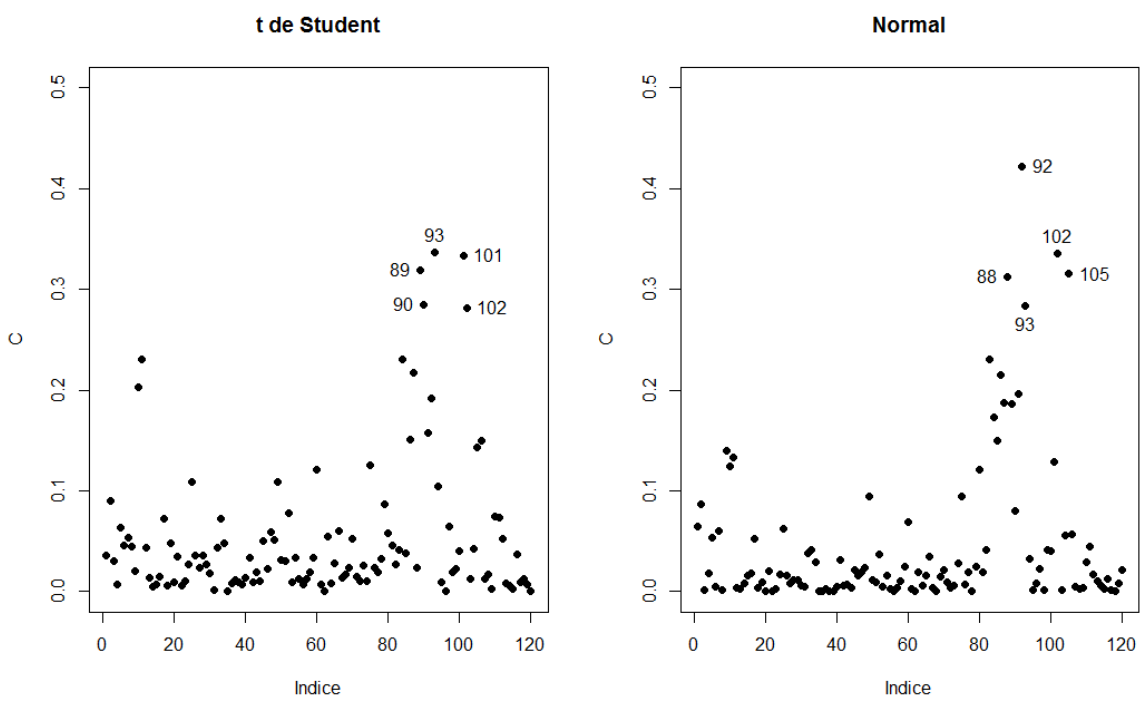

Figura 5.22: Gráficos de índices $C_{i}$ para $\boldsymbol{\theta}$ sob o esquema de ponderação de casos para o ajustes dos modelos parcialmente lineares com erros autoregressivos AR(1) t de Student e normal.
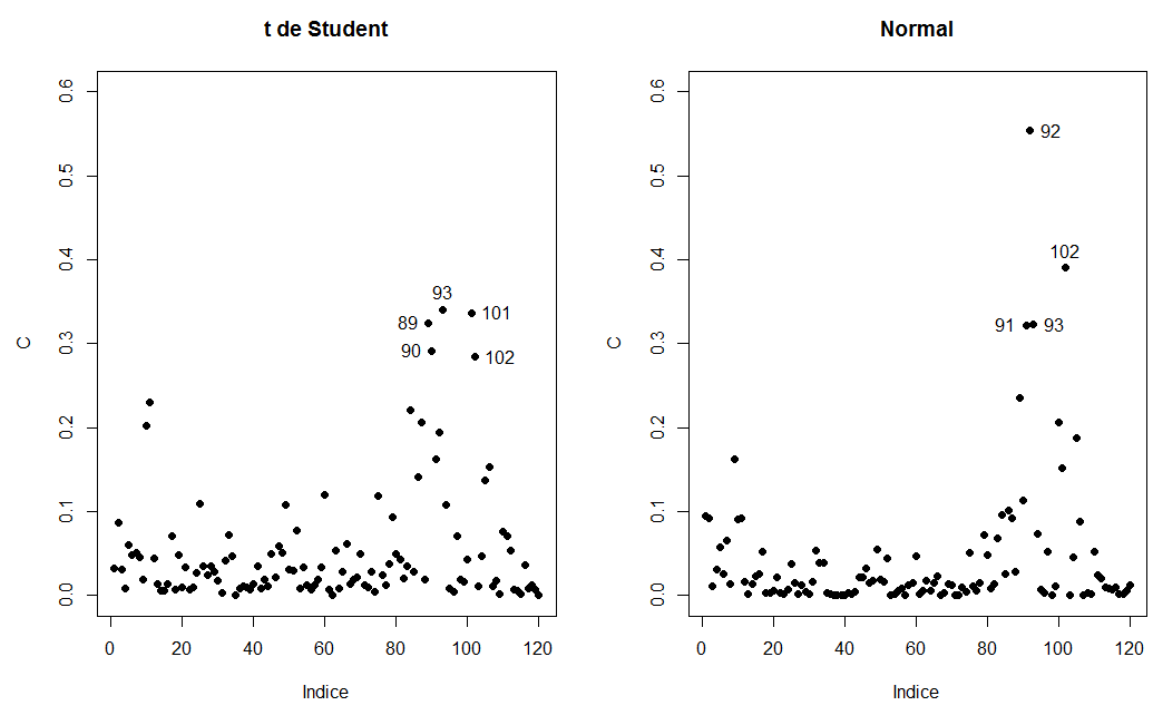

Figura 5.23: Gráficos de índices $C_{i}$ para $\boldsymbol{\beta}$ sob o esquema de ponderação de casos para o ajustes dos modelos parcialmente lineares com erros autoregressivos $A R(1)$ t de Student e normal. 

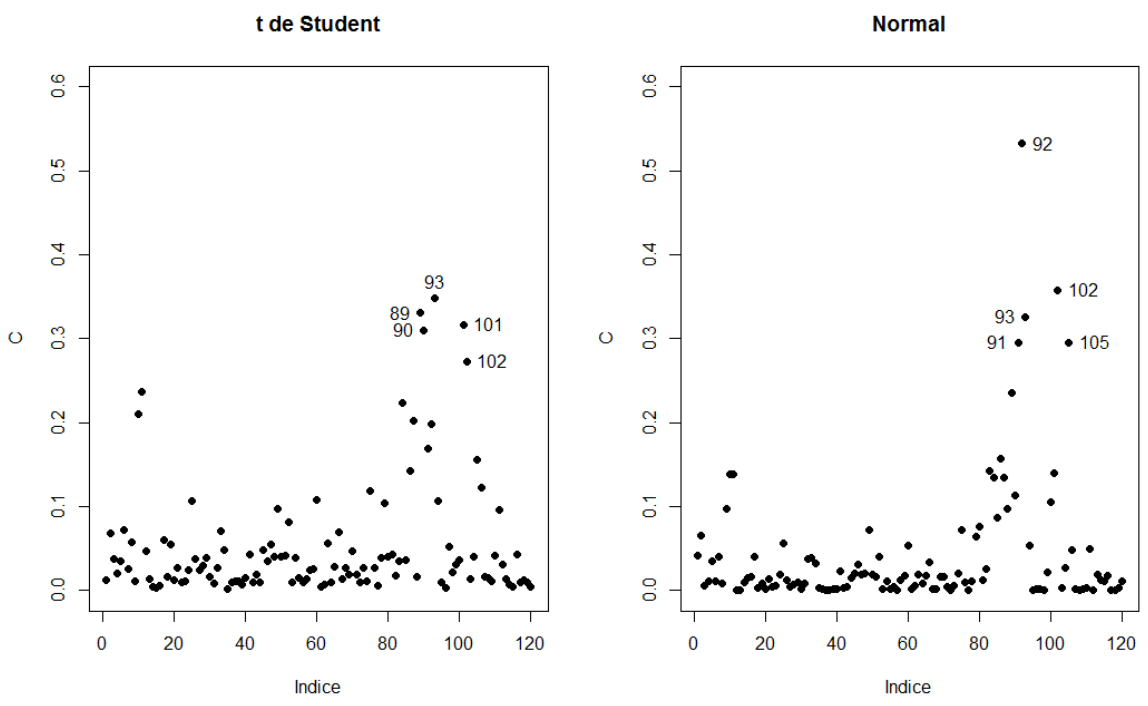

Figura 5.24: Gráficos de índices $C_{i}$ para $\mathbf{f}$ sob o esquema de ponderação de casos para o ajustes dos modelos parcialmente lineares com erros autoregressivos AR(1) $t$ de Student e normal.
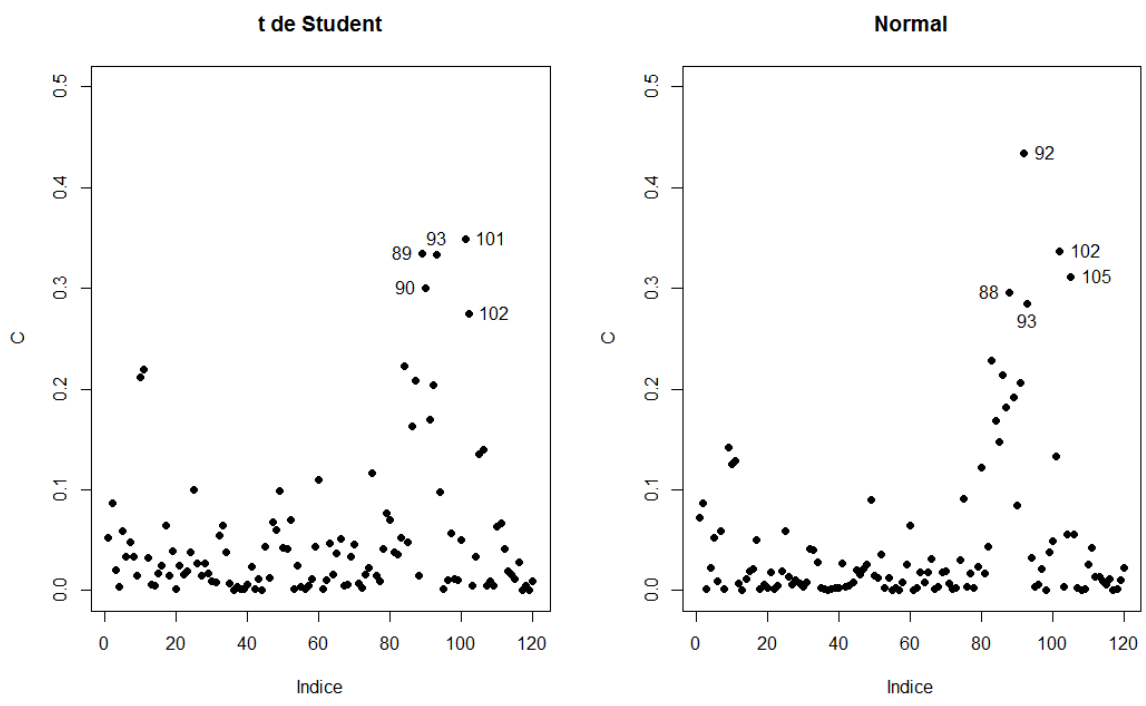

Figura 5.25: Gráficos de índices $C_{i}$ para $\phi$ sob o esquema de ponderação de casos para o ajustes dos modelos parcialmente lineares com erros autoregressivos AR(1) t de Student e normal. 

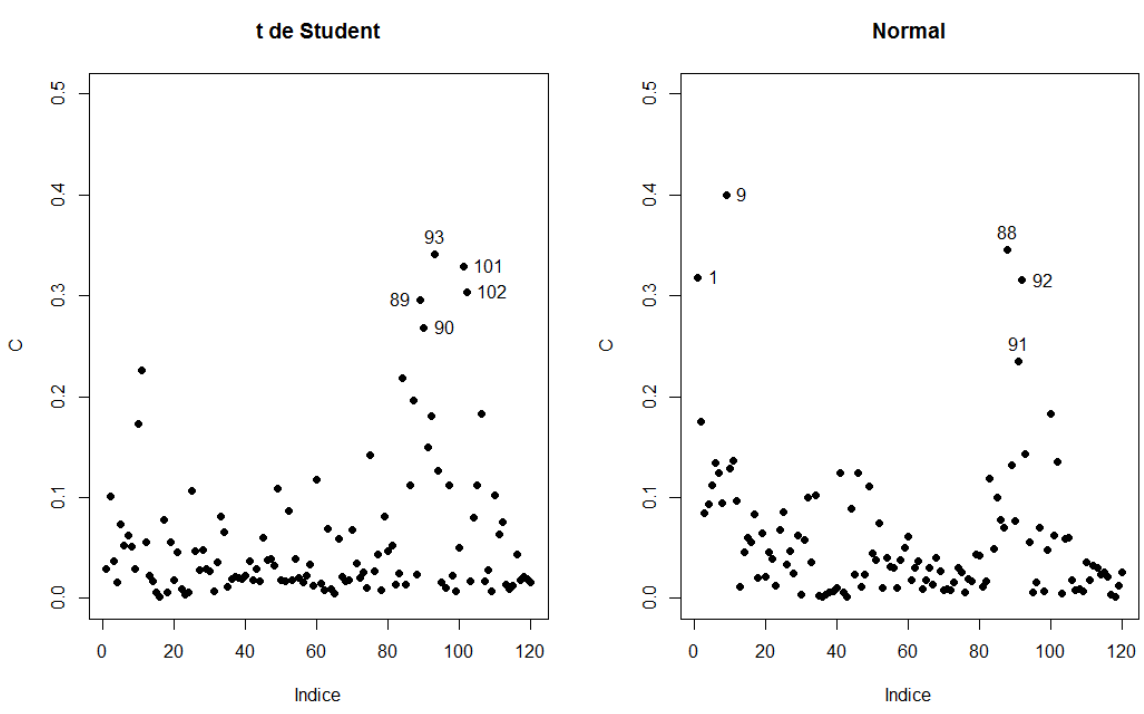

Figura 5.26: Gráficos de indices $C_{i}$ para $\rho$ sob o esquema de ponderação de casos para o ajustes dos modelos parcialmente lineares com erros autoregressivos AR(1) $t$ de Student e normal.

é nítido, para ambos os casos, que as observações dos anos de 1983 e 1984, isto é, entre a nonagésima e a centésima observação, parecem ser mais sensíveis.

Com o intuito de avaliar a medida de influência local, com esse mesmo esquema de perturbação, para diferentes conjuntos de $\boldsymbol{\theta}$, construímos as Figuras $5.23,5.24,5.25$ e 5.26 representando a influência de cada observação para $\boldsymbol{\beta}$, f, $\phi$ e $\rho$ respectivamente.

Por meio destas figuras, é nítido que o ajuste da t de Student apresenta estimativas mais robustas, analisando as medidas de influência para este esquema de perturbação, em comparação com o ajuste de normal para todos os subconjuntos de $\boldsymbol{\theta}$ analisados. Também, é evidente que as observações por volta dos anos de 1983 e 1984 influenciam a estimação de todos os parâmetros.

Nas Figura 5.27 e 5.28, apresentamos novamente os resíduos padronizados ao longo do tempo e o gráfico de dispersão entre as temperaturas marcando os pontos que se destacam dos demais. Notamos que as observações comentadas anteriormente também se destacam nestes gráficos, isto é, são observações que apresentam um alto resíduo padronizado e que destoam da relação linear facilmente visível, na Figura 5.28, entre as temperaturas. 

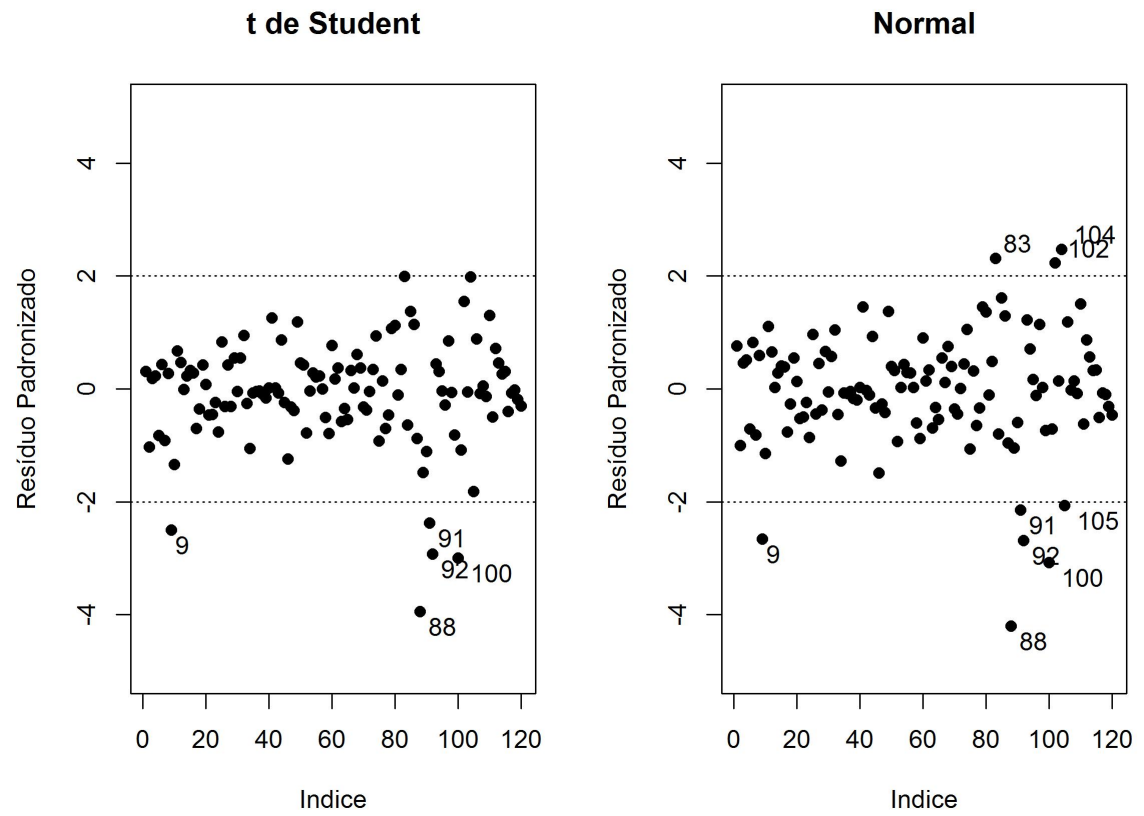

Figura 5.27: Gráficos dos resíduos padronizados pela ordem das observações para o ajustes dos modelos parcialmente lineares com erros autoregressivos AR(1) normal e $t$ de Student. 


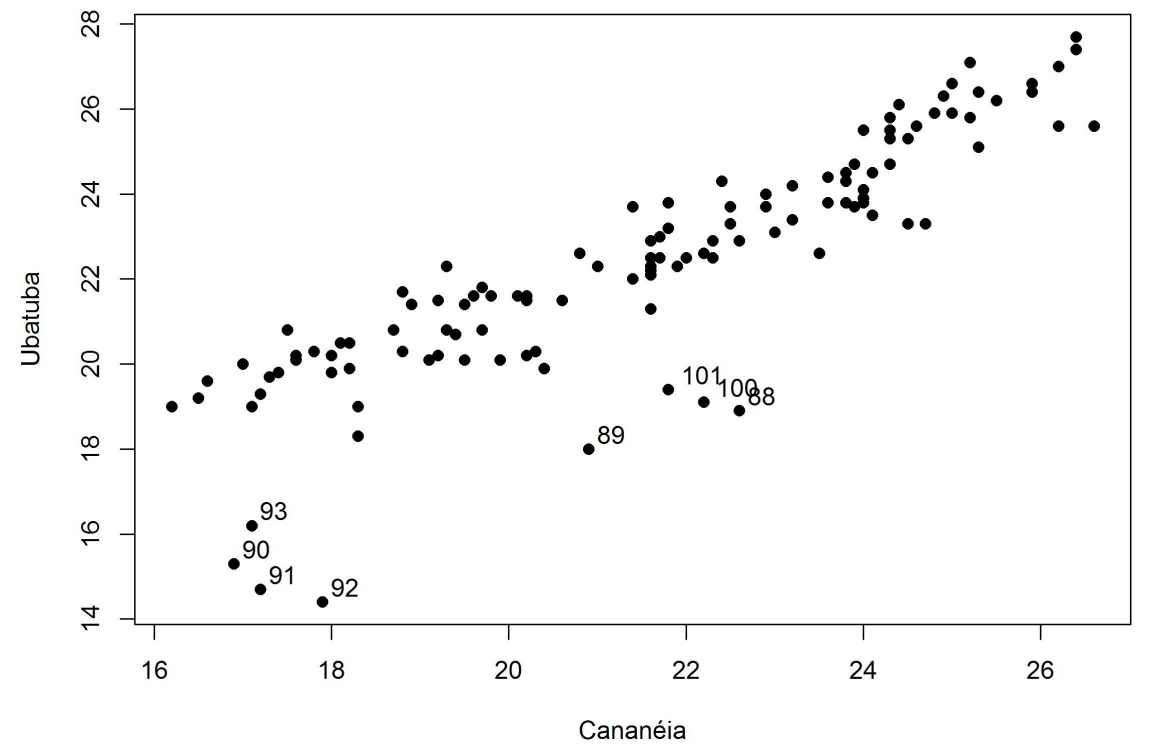

Figura 5.28: Gráfico de dispersão entre as temperaturas de Ubatuba e Cananéia destacando os pontos aberrantes. 


\subsubsection{Análise confirmatória}

Na presença de observações influentes, como detectadas na seção anterior, é comum ajustar novamente o modelo sem estas unidades e verificar se isto causa mudanças inferenciais importantes. No entanto, como estamos analisando séries de tempo, não faz muito sentido estimarmos o modelo sem uma observação no meio da série. Para contornar tal problema, como as observações detectadas como influentes estão no final da série (anos de 1983 e 1984), decidimos reestimar o modelo utilizando apenas os 7 primeiros anos de dados, ou seja, consideramos apenas as primeiras 84 observações.

Na Figura 5.29, mostramos novamente o gráfico de dispersão entre as temperaturas somente utilizando esse subconjunto dos dados. Fica evidente que a relação linear entre as séries é muito mais comportada.

A fim de detectar possíveis alterações nos resultados inferencias e comparar a sensibilidade dos ajustes supondo erros normais e t de Student, reestimamos os dois modelos com esse subconjunto menor dos dados.

As tabelas 5.2 e 5.3 nos mostram os intervalos de confiança das estimativas da parte paramétrica considerando todo o conjunto de dados, o conjunto menor e a variação percentual das estimativas comparando-se esses dois conjuntos. A tabela 5.2 apresenta os resultados supondo erros normais enquanto que a 5.3 nos mostra considerando erros t de Student.

Tabela 5.2: Intervalos de confiança de $95 \%$ para as estimativas da parte paramétrica considerando todos os dados e um subconjunto menor, supondo erros normais. LI - Limite inferior, LS - Limite superior

\begin{tabular}{r|cc|cc|c}
\hline & \multicolumn{4}{|c}{ Dados Completos } & \multicolumn{3}{c}{ Dados incompletos } & Var. \% \\
\hline & LI & LS & LI & LS & \\
\hline Intercepto & 4,5 & 4,79 & 5,67 & 5,93 & $24,8 \%$ \\
Cananéia & 0,818 & 0,832 & 0,788 & 0,80 & $3,8 \%$ \\
$\sigma^{2}$ & 0,535 & 0,568 & 0,280 & 0,299 & $45,7 \%$ \\
$\rho$ & 0,555 & 0,581 & 0,275 & 0,323 & $49 \%$ \\
\hline
\end{tabular}

Podemos ver que tanto supondo erros normais quanto t de Student, os intervalos de confiança de todos os parâmetros se alteram, evidenciando que de fato as observações marcadas como influentes causam mudanças significativas nas inferências encontradas. No entanto, analisando a variação percentual das estimativas encontradas, fica claro que o modelo supondo erros t de Student é menos sensível a tais mudanças, visto que as mudanças percentuais são relativamente menores. 


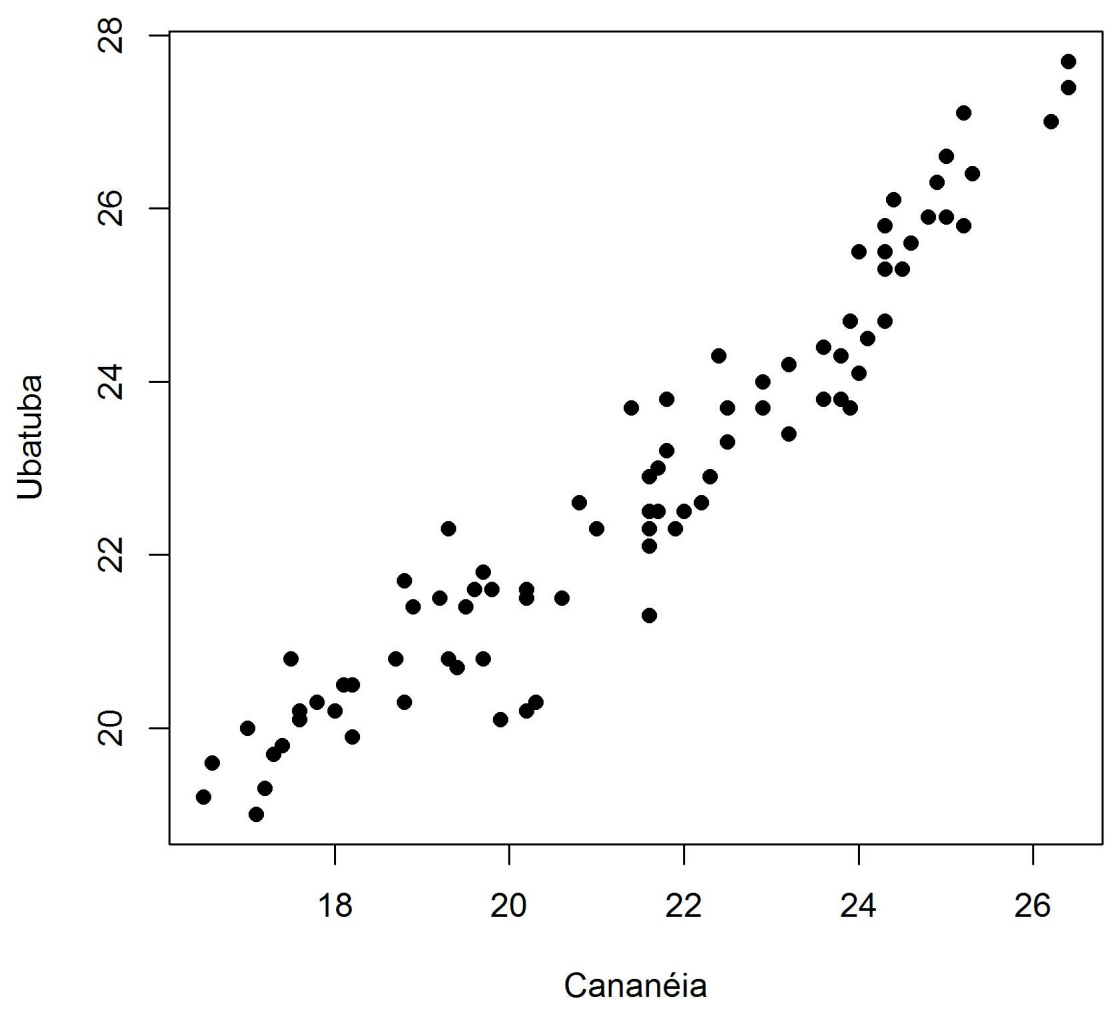

Figura 5.29: Gráfico de dispersão entre as temperaturas médias mensais de Ubatuba e Cananéia apenas para os 7 primeiros anos de dados.

Tabela 5.3: Intervalos de confiança de 95\% para as estimativas da parte paramétrica considerando todos os dados e um subconjunto menor, supondo erros $t$ de Student. LI - Limite inferior, LS - Limite superior

\begin{tabular}{r|cc|cc|c}
\hline & \multicolumn{4}{|c}{ Dados Completos } & \multicolumn{2}{c}{ Dados incompletos } & Var. \% \\
\hline & LI & LS & LI & LS & \\
\hline Intercepto & 5,06 & 5,28 & 5,79 & 6,03 & $14,2 \%$ \\
Cananéia & 0,801 & 0,813 & 0,785 & 0,796 & $2,05 \%$ \\
$\sigma^{2}$ & 0,70 & 0,74 & 0,463 & 0,509 & $32,2 \%$ \\
$\rho$ & 0,46 & 0,48 & 0,26 & 0,295 & $40,7 \%$ \\
\hline
\end{tabular}


Comparando as duas tabelas, podemos notar que para o conjunto de dados menor, as estimativas produzidas pelas duas distribuições de erros são muito mais similares ao compararmos esses valores para os dados completos, evidenciando a grande sensibilidade do ajuste supondo normalidade.

De todos os pontos apresentados neste capítulo, pudemos perceber, para esta aplicação, que controlar o efeito do tempo é importante e necessário. Ainda, ficou evidente que o uso de uma distribuição com caudas mais pesadas do que a normal se mostrou mais adequada, a fim de reduzir a sensibilidade do ajuste a pontos aberrantes e com isso produzir resultados inferenciais mais corretos.

Na próxima seção, discutiremos brevemente algumas idéias de como realizar previsões utilizando o modelo desenvolvido neste trabalho.

\subsection{Previsão}

O modelo apresentado neste trabalho foi desenvolvido com o intuito de realizar inferências sobre a relação entre as variáveis, no entanto, o mesmo pode ser utilizado para realizar previsões.

Para o modelo definido na Seção 2.3, a previsão para $h$ passos a frente, quando se é conhecido a série até o instante $t$, é comumente denotada por $\hat{y}_{t}(h)$, e é definida como

$$
\hat{y}_{t}(h)=\hat{\beta}_{0}+\hat{\beta}_{1} x_{t+h}+\rho^{(h)} \hat{\epsilon}_{t},
$$

em que $\hat{\epsilon}_{t}$ é conhecida, visto que temos os valores observados até o instante $t$. Lembrando que o valor de $x_{t+h}$ tem que ser conhecido.

Portanto, para o modelo estudado neste trabalho, a previsão para $h$ passos a frente é dada da mesma forma, ou seja,

$$
y_{t} \hat{(h)}=\mathbf{x}_{t+h}^{\top} \hat{\boldsymbol{\beta}}+\hat{f}\left(t_{t+h}\right)+\rho^{(h)} \hat{\epsilon}_{t} .
$$

Para estudos longitudionais, em que o estudo é realizado sempre no mesmo intervalo de tempo, não há problemas em encontrar $\hat{f}\left(t_{t+h}\right)$, visto que este valor já foi estimado com outras observações. No entanto, em séries de tempo, não temos $\hat{f}\left(t_{t+h}\right)$, pois esta estimativa não foi necessária anteriormente. Logo, o maior problema para encontrar a previsão é obter justamente este valor.

Conforme explicamos anteriormente, o tipo de spline utilizado neste projeto é linear após o último ponto. Desta forma, uma alternativa para prever $\hat{f}\left(t_{t+h}\right)$ seria utilizar esta função linear, todavia, após muitos passos a frente, essa fun- 
ção linear pode não fazer sentido. Maiores detalhes de como obter essa função linear pelas estimativas encontradas podem ser vistos em Green e Silverman [1994].

Outra alternativa seria obter um modelo entre o efeito não paramétrico estimado e a variável, no caso, o tempo. Podemos construir uma relação do tipo quadrática ou cúbica ou um polinômio de ordem maior que se ajuste bem aos dados e assim utilizar este ajuste para encontrar $\hat{f}\left(t_{t+h}\right)$. Modelos de séries de tempo também podem ser construídos, como por exemplo, modelos do tipo Box-Jenkins (Hamilton [1994]).

A fim de avaliar as previsões, consideramos apenas o método de utilizar a função não paramétrica de forma linear. Assim, para as mesmas séries de temperaturas, estimamos o modelo parcialmente linear com erros autoregressivos t de Student apenas com as primeiras 110 observações, deixando as 10 restantes para avaliarmos o poder preditivo do modelo.

A Figura 5.30 nos mostra os valores observados da série, os valores estimados para os dados utilizados no ajuste do modelo e os valores previstos para pontos não utilizados no ajuste. A linha tracejada em verde indica até qual observação foi utilizada na estimação dos parâmetros do modelo. Podemos ver pelas linhas em vermelho e em preto que a série estimada é muito próxima da série observada. Já para os dados previstos, é possível notar que para os primeiros pontos, a previsão continua muito boa, mas vai perdendo seu poder de predição à medida em que aumentamos a janela das previsões. Este fato já é conhecido na previsão de modelos com erros autoregressivos, porém, é atenuado para o modelo em que estamos estudando, devido à aproximação linear do spline também perder seu poder predito à medida em aumentamos esta janela de previsão.

Portanto, novas formas de previsão podem ser estudadas a fim de aperfeiçoálas.

\subsection{Conclusões do capítulo}

Neste capítulo, aplicamos o modelo desenvolvido neste trabalho, comparandoo com um método mais simples, ficando nítida a adequação do modelo estudado, para o caso apresentado. Mostramos a importância de controlar o efeito de determinada variável de forma não paramétrica e que, ao utilizarmos uma distribuição com caudas mais pesadas do que a normal, os resultados parecem ser mais robustos. Por fim, apresentamos formas de realizar previsões, tornando evidente que este ponto pode ser melhor estudado. 


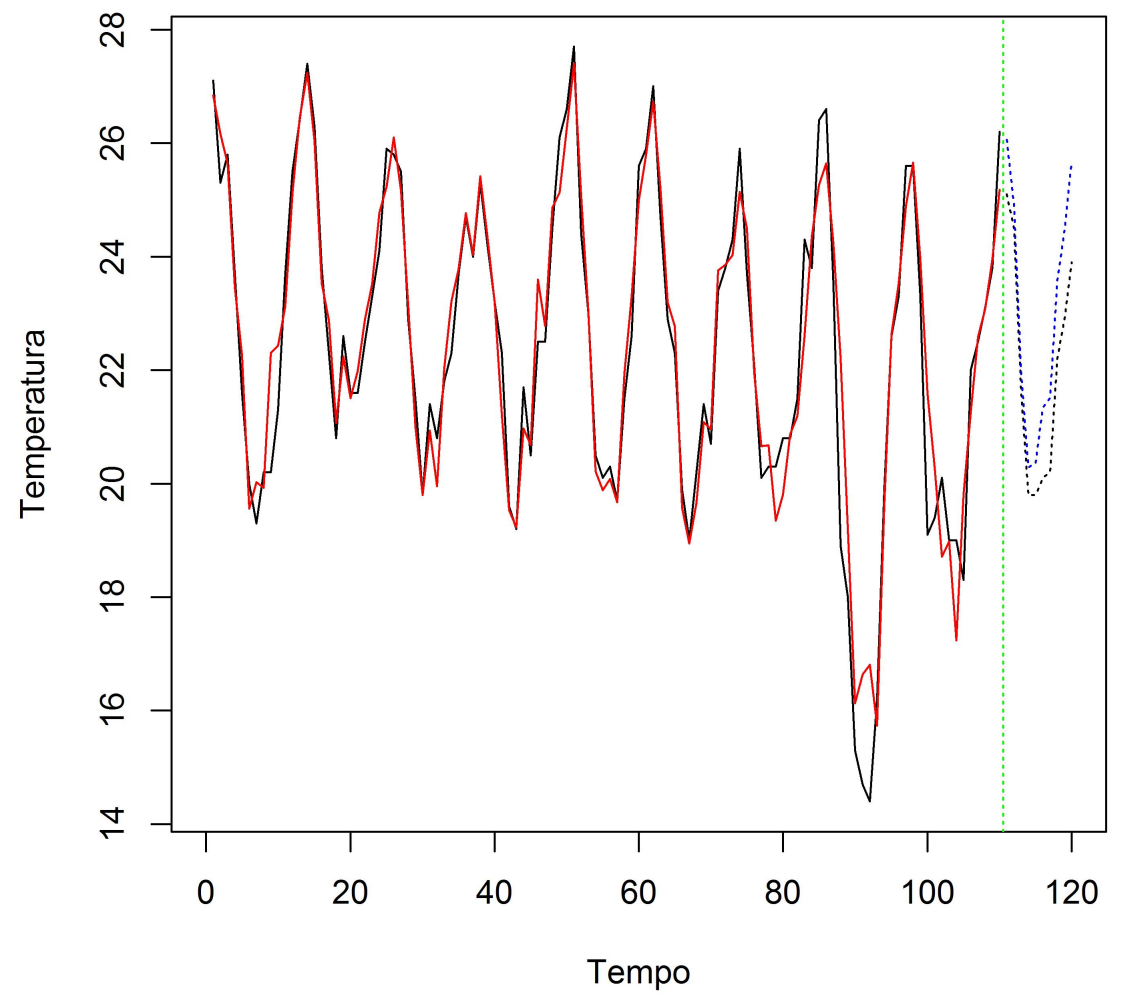

Figura 5.30: Série observada de temperatura de Ubatuba (em preto); série estimada pelo modelo (em vermelho); valores previstos para a série de temperaturas (em azul pontilhado); valores observados não utilizados no processo de estimação (em preto pontilhado); a linha verde pontilhada indica o ponto que foi utilizado no ajuste do modelo. 
100 APLICAÇÕES 


\section{Capítulo 6}

\section{Conclusões finais}

\subsection{Considerações finais}

Este trabalho generaliza os modelos parcialmente lineares simétricos incluindo um parâmetro a mais, representando a estrutura de autocorrelação dos erros que supostamente apresenta uma relação autoregressiva de primeira ordem.

As maiores contribuições desta dissertação estão presentes nos Capítulos 3 e 4. No Capítulo 3 encontramos a função de verossimilhança do modelo, bem como algumas medidas para realizarmos a estimação e as inferências das estimativas, como as funções escore e a matriz de Fisher. Resultados assintóticos também são apresentados, assim como um processo iterativo para a estimação dos parâmetros do modelo é discutido. Já no Capítulo 4, apresentamos uma análise de diagnóstico do modelo, mais precisamente definimos o resíduo padronizado, os pontos de alavanca generalizados e encontramos medidas de influência local para três esquemas de perturbação diferentes.

No Capítulo 5 aplicamos o modelo estudado para duas séries de temperatura medidas ao longo do tempo, em que é possível mostrar que a presença dos resíduos autoregressivos é extremamente importante, bem como a utilização de uma distribuição com caudas mais pesadas do que a normal e controlar o efeito do tempo de modo não paramétrico. Notamos que o ajuste do modelo é superior ao utilizarmos uma distribuição com caudas mais pesadas do que a normal, no caso a t de Student. Trata-se de um modelo com estimativas mais robustas no sentido de fazer com que observações aberrantes tenham um peso menor nas estimativas.

É possível notar que o modelo desenvolvido neste trabalho pode ser ex- 
tremamente útil para experimentos longitudinais, em que a presença de autocorrelação é muito usual, principalmente a correlação entre observações consecutivas. Com os modelos parcialmente lineares simétricos $\mathrm{AR}(1)$ é possível também incluir variáveis de maneira não paramétrica, ou seja, não é preciso definir a priori a relação entre a variável resposta e as variáveis explicativas.

\subsection{Trabalhos futuros}

Como possíveis trabalhos futuros, várias generalizações podem ser estudadas. A primeira, e talvez a mais trivial, é considerar várias funções não paramétricas no modelo ao invés de apenas uma. Outra possível contribuição seria considerar a possibilidade de incluir interações entre as funções não paramétricas. Não apresentamos neste trabalho modelos não paramétricos com interações, no entanto, este tipo de modelo é conhecido na literatura.

Ainda, é possível generalizar a estrutura de correlação dos erros, como, por exemplo, supondo que os erros obedeçam a um processo $\operatorname{AR}(p)$ com $p$ maior igual a 1 ao invés de apenas um autoregressivo de ordem 1 . De forma geral, é possível considerar os erros como um processo $\operatorname{ARMA}(p, q)$. Ainda, podemos considerar estruturas do tipo ARCH, GARCH para casos em que a variância dos erros não é constante. Esse tipo de erro pode ser utilizado em estudos de séries temporais financeiras, em que há frequentemente a presença de forte volatilidade nos dados.

Outra alternativa de trabalho futuro seria generalizar a distribuição dos erros para outras classes não necessariamente simétricas, como, por exemplo, considerando a normal assimétrica.

Em relação a análise de diagnóstico, outras medidas frequentemente utilizadas podem ser construídas, além de podermos encontrar as medidas de influência local para outros esquemas de perturbação de interese. Assim, também podemos considerar, ao invés da curvatura normal, o método da curvatura conformal. Maiores detalhes podem ser encontrados em Medeiros [2006]. 


\section{Apêndice A}

\section{Derivação dos momentos impostos pelo modelo}

Neste apêndice, derivaremos os dois primeiros momentos impostos pelo modelo definido no Capítulo 3. A correlação entre observações também será calculada.

\section{A.1 Derivação dos dois primeiros momentos}

Iremos derivar os dois primeiros momentos da variável resposta do modelo definido no Capítulo 3. Similarmente aos modelos simétricos $\mathrm{AR}(1)$ (vide Cao et al. [2010]) vamos supor que $y_{i} \mid y_{i-1} \sim \mathrm{S}\left(\mu_{i}, \phi\right)$, com $\mu_{i}=\mathbf{x}_{i}^{\top} \boldsymbol{\beta}+\boldsymbol{\eta}_{i}^{\top} \mathbf{f}+$ $\rho\left(y_{i-1}-\mathbf{x}_{i-1}^{\top} \boldsymbol{\beta}-\boldsymbol{\eta}_{i-1}^{\top} \mathbf{f}\right)$.

Logo, obtemos

$$
E\left[y_{i}\right]=E\left[E\left[y_{i} \mid y_{i-1}\right]\right]=E\left[\mu_{i}\right]=\mathbf{x}_{i}^{\top} \boldsymbol{\beta}+\boldsymbol{\eta}_{i}^{\top} \mathbf{f}+\rho\left(E\left[y_{i-1}\right]-\mathbf{x}_{i-1}^{\top} \boldsymbol{\beta}-\boldsymbol{\eta}_{i-1}^{\top} \mathbf{f}\right) .
$$

Além disso, como $\epsilon_{0}=0$, temos $y_{1}=\mathbf{x}_{1}^{\top} \boldsymbol{\beta}+\boldsymbol{\eta}_{1}^{\top} \mathbf{f}+e_{1}$. Portanto, $E\left[y_{1}\right]=$ $\mathbf{x}_{1}^{\top} \boldsymbol{\beta}+\boldsymbol{\eta}_{1}^{\top} \mathbf{f}$. Então,

$$
E\left[y_{2}\right]=\mathbf{x}_{2}^{\top} \boldsymbol{\beta}+\boldsymbol{\eta}_{2}^{\top} \mathbf{f}+\rho\left(\mathbf{x}_{1}^{\top} \boldsymbol{\beta}+\boldsymbol{\eta}_{1}^{\top} \mathbf{f}-\mathbf{x}_{1}^{\top} \boldsymbol{\beta}-\boldsymbol{\eta}_{1}^{\top} \mathbf{f}\right)=\mathbf{x}_{2}^{\top} \boldsymbol{\beta}+\boldsymbol{\eta}_{2}^{\top} \mathbf{f}
$$

Com isso, podemos denotar que

$$
E\left[y_{k}\right]=\mathbf{x}_{k}^{\top} \boldsymbol{\beta}+\boldsymbol{\eta}_{k}^{\top} \mathbf{f}, \quad \forall k
$$


Vamos agora obter a variância de $y_{i}$. Temos que

$$
\begin{aligned}
& \operatorname{Var}\left[y_{i}\right]=\operatorname{Var}\left(E\left[y_{i} \mid y_{i-1}\right]\right)+E\left(\operatorname{Var}\left[y_{i} \mid y_{i-1}\right]\right) \\
& =E[\phi \xi]+\operatorname{Var}\left(\mathbf{x}_{i}^{\top} \boldsymbol{\beta}+\boldsymbol{\eta}_{i}^{\top} \mathbf{f}+\rho\left(y_{i-1}-\mathbf{x}_{i-1}^{\top} \boldsymbol{\beta}-\boldsymbol{\eta}_{i-1}^{\top} \mathbf{f}\right)\right)=\phi \xi+\rho^{2} \operatorname{Var}\left(y_{i-1}\right) .
\end{aligned}
$$

Podemos ver claramente que $\operatorname{Var}\left(y_{1}\right)=\xi \phi$. Logo, $\operatorname{Var}\left(y_{2}\right)=\xi \phi+\rho^{2} \xi \phi$. Então, com sucessivas substituições, temos, para qualquer $k$

$$
\operatorname{Var}\left[y_{k}\right]=\xi \phi \sum_{j=0}^{k-1} \rho^{2 j} .
$$

Além de $E\left[y_{k}\right]$ e $\operatorname{Var}\left[y_{k}\right]$, também podemos derivar as covariâncias entre duas observações, conforme mostramos na próxima subseção.

\section{A.2 Derivação de correlação}

Para obtermos as covariâncias entre diferentes observações, primeiramente iremos calcular $E\left[y_{j} y_{k}\right], j \neq k$. Temos que

$$
\begin{aligned}
& E\left[y_{j} y_{k}\right]=E\left\{\left(\left[\mathbf{x}_{j}^{\top} \boldsymbol{\beta}+\boldsymbol{\eta}_{j}^{\top} \mathbf{f}+\rho\left[y_{j-1}-\mathbf{x}_{j-1}^{\top} \boldsymbol{\beta}-\boldsymbol{\eta}_{i-1}^{\top} \mathbf{f}\right]+e_{j}\right]\right)\right. \\
& \left.\left(\left[\mathbf{x}_{k}^{\top} \boldsymbol{\beta}+\boldsymbol{\eta}_{k}^{\top} \mathbf{f}+\rho\left[y_{k-1}-\mathbf{x}_{k-1}^{\top} \boldsymbol{\beta}-\boldsymbol{\eta}_{k-1}^{\top} \mathbf{f}\right]+e_{k}\right]\right)\right\} \\
& =\left(\mathbf{x}_{j}^{\top} \boldsymbol{\beta}+\boldsymbol{\eta}_{j}^{\top} \mathbf{f}\right)\left(\mathbf{x}_{k}^{\top} \boldsymbol{\beta}+\boldsymbol{\eta}_{k}^{\top} \mathbf{f}\right)+\rho^{2} E\left\{\left[y_{j-1}-\mathbf{x}_{j-1}^{\top} \boldsymbol{\beta}-\boldsymbol{\eta}_{j-1}^{\top} \mathbf{f}\right]\left[y_{k-1}-\mathbf{x}_{k-1}^{\top} \boldsymbol{\beta}-\boldsymbol{\eta}_{k-1}^{\top} \mathbf{f}\right]\right\}+ \\
& +\rho\left(E\left[y_{j-1} e_{k}\right]+E\left[y_{k-1} e_{j}\right]\right) \\
& =E\left[y_{k}\right] E\left[y_{j}\right]+\rho\left(E\left[y_{j-1} e_{k}\right]+E\left[y_{k-1} e_{j}\right]\right)+\rho^{2} \operatorname{Cov}\left(y_{k-1}, y_{j-1}\right) .
\end{aligned}
$$

Logo, obtemos

$$
\begin{aligned}
& \operatorname{Cov}\left(y_{k}, y_{j}\right)=\rho\left(E\left[y_{j-1} e_{k}\right]+E\left[y_{k-1} e_{j}\right]\right)+\rho^{2} \operatorname{Cov}\left(y_{k-1}, y_{j-1}\right) \\
& = \begin{cases}\rho^{2} \operatorname{Cov}\left(y_{k-1}, y_{j-1}\right)+\rho \xi \phi, & \text { se }|j-k|=1 \\
\rho^{2} \operatorname{Cov}\left(y_{k-1}, y_{j-1}\right), & \text { se }|j-k|>1\end{cases}
\end{aligned}
$$

Pela expressão de $y_{1}$, podemos obter a covariância entre $y_{1}$ e $y_{k}$ para qualquer $k$. Com isso, encontramos iterativamente a covariância entre $y_{k}$ e $y_{j}$, $\forall k \neq j$. 
Analisando a covariância entre duas observações, podemos destacar alguns pontos relevantes. Primeiramente, notamos uma correlação maior entre observações consecutivas, se $\rho$ for maior ou igual a 0 . Ainda, como $\rho$ é menor do que 1, observamos que, ao realizar o processo iterativo para obter a covariância, esta, para pontos distantes do início da série, tornar-se-á cada vez mais próxima de 0 à medida que a distância aumentar e as observações não forem consecutivas. Isto pode ser mais facilmente visualizado por meio das relações abaixo:

Para $|j-k|>1$

$$
\begin{array}{r}
\operatorname{Cov}\left(y_{k}, y_{j}\right)=\rho^{2} \operatorname{Cov}\left(y_{k-1}, y_{j-1}\right)=\rho^{4} \operatorname{Cov}\left(y_{k-2}, y_{j-2}\right)=\cdots= \\
=\rho^{2[\min (k, j)-1]} \operatorname{Cov}\left(y_{1}, y_{[|j-k|+1]}\right) .
\end{array}
$$

Já, para $|j-k|=1$,

$$
\begin{array}{r}
\operatorname{Cov}\left(y_{k}, y_{k+1}\right)=\rho^{2} \operatorname{Cov}\left(y_{k-1}, y_{k}\right)+\rho \xi \phi= \\
=\rho^{4} \operatorname{Cov}\left(y_{k-2}, y_{k-1}\right)+\rho^{3} \xi \phi+\rho \xi \phi=\rho^{2[k-1]} \operatorname{Cov}\left(y_{1}, y_{2}\right)+\sum_{i=0}^{k-2} \rho^{(2 i+1)} \xi \phi
\end{array}
$$

Como a equação da covariância entre observações sucessivas só depende da covariância entre a primeira e a segunda observação, iremos calcular este valor para obtermos uma fórmula fechada. Temos que

$$
\begin{array}{r}
E\left[y_{1} y_{2}\right]=E\left[\left(\mathbf{x}_{1}^{\top} \beta+\boldsymbol{\eta}_{1}^{\top} \mathbf{f}+e_{1}\right)\left(\mathbf{x}_{2}^{\top} \beta+\boldsymbol{\eta}_{2}^{\top} \mathbf{f}+\rho\left\{y_{1}-\mathbf{x}_{1}^{\top} \beta-\boldsymbol{\eta}_{1}^{\top} \mathbf{f}\right\}+e_{2}\right)\right] \\
=E\left(y_{1}\right) E\left(y_{2}\right)+\rho E\left(y_{1} e_{1}\right)=E\left(y_{1}\right) E\left(y_{2}\right)+\rho \xi \phi .
\end{array}
$$

Logo,

$$
\operatorname{Cov}\left(y_{1}, y_{2}\right)=\rho \xi \phi \quad \text { e } \operatorname{Cov}\left(y_{k}, y_{k+1}\right)=\sum_{i=0}^{k-1} \rho^{(2 i+1)} \xi \phi
$$




\section{Apêndice B}

\section{Funções escore}

Neste apêndice derivamos a função de verossimilhança penalizada, definida no Capítulo 3, em relação a cada parâmetro. O logaritmo da função de verossimilhança penalizada, apresentado também no Capítulo 3, é denotado por

$$
L_{\mathrm{p}}(\boldsymbol{\theta})=-\frac{n}{2} \log \phi+\sum_{i=1}^{n} \log \left\{g\left(u_{i}\right)\right\}-\frac{\alpha}{2} \mathbf{f}^{\top} \mathbf{K} \mathbf{f}
$$

em que,

$$
u_{i}=\frac{\left(\epsilon_{i}-\rho \epsilon_{i-1}\right)^{2}}{\phi}, \epsilon_{i}=y_{i}-\mathbf{x}_{i}^{\top} \boldsymbol{\beta}-\boldsymbol{\eta}_{i}^{\top} \mathbf{f} e \epsilon_{0}=0
$$

Portanto, através de propriedades básicas de derivação, obtemos

$$
\begin{aligned}
U_{\phi} & =\frac{\partial L_{\mathrm{p}}(\boldsymbol{\theta})}{\partial \phi}=-\frac{n}{2 \phi}-\sum_{i=1}^{n} W_{g}\left(u_{i}\right) \frac{u_{i}}{\phi} \mathrm{e} \\
U_{\beta_{j}} & =\frac{\partial L_{\mathrm{p}}(\boldsymbol{\theta})}{\partial \beta_{j}}=-\sum_{i=1}^{n} W_{g}\left(u_{i}\right) \frac{2}{\phi}\left(\epsilon_{i}-\rho \epsilon_{i-1}\right)\left(x_{i j}-\rho x_{(i-1) j}\right),(j=1, \ldots, p) .
\end{aligned}
$$

Do mesmo modo, encontramos 


$$
\begin{aligned}
& U_{f_{j}}=\frac{\partial L_{\mathrm{p}}(\boldsymbol{\theta})}{\partial f_{j}}=-\sum_{i=1}^{n}\left\{W_{g}\left(u_{i}\right) \frac{2}{\phi}\left(\epsilon_{i}-\rho \epsilon_{i-1}\right)\left(\eta_{i j}-\rho \eta_{(i-1) j}\right)\right\}-[\alpha \mathbf{K} \mathbf{f}]_{j}, \\
&(j=1, \ldots, q) \mathrm{e} \\
& U_{\rho}=\frac{\partial L_{\mathrm{p}}(\boldsymbol{\theta})}{\partial \rho}=-\sum_{i=1}^{n} W_{g}\left(u_{i}\right) \frac{2}{\phi}\left(\epsilon_{i}-\rho \epsilon_{i-1}\right) \epsilon_{i-1},
\end{aligned}
$$

em que $[\alpha \mathbf{K f}]_{j}$ representa a $j$-ésima posição do vetor $\alpha \mathbf{K f}$. Já $W_{g}(u)$ é igual a $\frac{g^{\prime}(u)}{g(u)}, g(u)$ identificando a distribuição simétrica que está sendo utilizada. No Capítulo 2 apresentamos alguns valores de $W_{g}(u)$ para as principais distribuições.

Para facilitar o manuseio computacional destas funções, escrevê-la-emos em forma matricial. Para isso, utilizaremos as matrizes que foram definidas na Seção 3.2. Assim, notamos que podemos escrever as funções acima como

$$
\begin{aligned}
\mathbf{U}_{\beta} & =\frac{\partial L_{\mathrm{p}}(\boldsymbol{\theta})}{\partial \boldsymbol{\beta}}=(\mathbf{A X})^{\top} \mathbf{D}(\mathbf{v}) \phi^{-1} \mathbf{A} \boldsymbol{\epsilon}, \\
\mathbf{U}_{f} & =\frac{\partial L_{\mathrm{p}}(\boldsymbol{\theta})}{\partial \mathbf{f}}=(\mathbf{A N})^{\top} \mathbf{D}(\mathbf{v}) \phi^{-1} \mathbf{A} \boldsymbol{\epsilon}-\alpha \mathbf{K} \mathbf{f}, \\
U_{\phi} & =\frac{\partial L_{\mathrm{p}}(\boldsymbol{\theta})}{\partial \phi}=\frac{1}{2 \phi} \mathbf{1}_{n}^{\top}\left(\mathbf{D}(\mathbf{m}) \mathbf{1}_{n}-\mathbf{1}_{n}\right) \mathrm{e} \\
U_{\rho} & =\frac{\partial L_{\mathrm{p}}(\boldsymbol{\theta})}{\partial \rho}=-\phi^{-1} \boldsymbol{\epsilon}^{\top} \mathbf{B}^{\top} \mathbf{D}(\mathbf{v}) \mathbf{A} \boldsymbol{\epsilon} .
\end{aligned}
$$




\section{Apêndice C}

\section{Matriz de informação de Fisher}

\section{C.1 Matriz Hessiana}

Neste apêndice, apresentamos os cálculos para a obtênção da matriz observada de Fisher. Encontramos primeiramente em forma analítica para depois escrevermos em notação matricial, usando as matrizes definidas anteriormente.

Para o parâmetro $\boldsymbol{\beta}$, temos que

$$
\begin{aligned}
\ddot{L}_{\beta_{j} \beta_{k}} & =\frac{\partial^{2} L_{p}(\boldsymbol{\theta})}{\partial \beta_{j} \partial \beta_{k}}=\frac{4}{\phi} \sum_{i=1}^{n}\left\{W_{g}^{\prime}\left(u_{i}\right) u_{i}\left(x_{i j}-\rho x_{(i-1) j}\right)\left(x_{i k}-\rho x_{(i-1) k}\right)\right\}+ \\
+ & \frac{2}{\phi} \sum_{i=1}^{n}\left\{W_{g}\left(u_{i}\right)\left(x_{i j}-\rho x_{(i-1) j}\right)\left(x_{i k}-\rho x_{(i-1) k}\right)\right\},(j, h=1, \ldots, p) .
\end{aligned}
$$

Assim, em forma matricial, obtemos

$$
\ddot{\mathbf{L}}_{\beta \beta}=\frac{\partial^{2} L_{p}(\boldsymbol{\theta})}{\partial \boldsymbol{\beta} \partial \boldsymbol{\beta}^{\top}}=\frac{1}{\phi}\left[(\mathbf{A X})^{\top}(-\mathbf{D}(\mathbf{v})+4 \mathbf{D}(\mathbf{d}))(\mathbf{A X})\right] .
$$

Para os parâmetros f , o cálculo é similar ao dos parâmetros $\boldsymbol{\beta}$, apenas com a inclusão do termo referente à matriz de penalização, logo obtemos

$$
\ddot{\mathbf{L}}_{f f}=\frac{\partial^{2} L_{p}(\boldsymbol{\theta})}{\partial \mathbf{f} \partial \mathbf{f}^{\top}}=\frac{1}{\phi}\left[(\mathbf{A N})^{\top}(-\mathbf{D}(\mathbf{v})+4 \mathbf{D}(\mathbf{d}))(\mathbf{A N})\right]-\alpha \mathbf{K} .
$$

Em relação a $\phi$ e $\rho$, encontramos 


$$
\begin{array}{r}
\ddot{L}_{\phi \phi}=\frac{\partial^{2} L_{p}(\boldsymbol{\theta})}{\partial \phi^{2}}=\frac{n}{2 \phi^{2}}+\sum_{i=1}^{n} W_{g}^{\prime}\left(u_{i}\right) \frac{u_{i}^{2}}{\phi^{2}}+\sum_{i=1}^{n} W_{g}\left(u_{i}\right) \frac{2 u_{i}}{\phi^{2}}= \\
=\frac{1}{\phi^{2}}\left(\frac{n}{2}+\mathbf{u}^{\top} \mathbf{D}(\mathbf{c}) \mathbf{u}-\mathbf{u}^{\top} \mathbf{D}(\mathbf{v}) \mathbf{1}_{n}\right) \quad \mathrm{e} \\
\ddot{L}_{\rho \rho}=\frac{\partial^{2} L_{p}(\boldsymbol{\theta})}{\partial \rho^{2}}=\sum_{i=1}^{n} 4 W_{g}^{\prime}\left(u_{i}\right) \frac{u_{i} \epsilon_{i-1}^{2}}{\phi}+\sum_{i=1}^{n} 2 W_{g}\left(u_{i}\right) \frac{\epsilon_{i-1}^{2}}{\phi}= \\
=\frac{1}{\phi}\left[(\mathbf{B} \boldsymbol{\epsilon})^{\top}(-\mathbf{D}(\mathbf{v})+4 \mathbf{D}(\mathbf{d}))(\mathbf{B} \boldsymbol{\epsilon})\right] .
\end{array}
$$

Os termos acima são referentes à diagonal principal da matriz $\ddot{\mathbf{L}}_{\theta \theta}$. Agora, prosseguimos para o cálculo dos termos cruzados. Primeiramente mostramos os elementos de $\boldsymbol{\beta}$ em relação aos demais. Temos que

$$
\begin{array}{r}
\ddot{L}_{\beta_{j} f_{k}}=\frac{\partial^{2} L_{p}(\boldsymbol{\theta})}{\partial \beta_{j} \partial f_{k}}=\frac{1}{\phi} \sum_{i=1}^{n}\left(x_{i j}-\rho x_{(i-1) j}\right)\left(\eta_{i j}-\rho \eta_{(i-1) j}\right)\left[4 W_{g}^{\prime}\left(u_{i}\right) u_{i}+2 W_{g}\left(u_{i}\right)\right] \\
(j, h=1, \ldots, p),
\end{array}
$$

e em notação matricial

$$
\ddot{\mathbf{L}}_{\beta f}=\frac{\partial^{2} L_{p}(\boldsymbol{\theta})}{\partial \boldsymbol{\beta} \partial \mathbf{f}^{\top}}=\frac{1}{\phi}\left[(\mathbf{A X})^{\top}(-\mathbf{D}(\mathbf{v})+4 \mathbf{D}(\mathbf{d})) \mathbf{A N}\right] .
$$

Já, para os termos cruzados de $\boldsymbol{\beta}$ com $\phi$ e $\rho$, obtemos

$$
\begin{gathered}
\ddot{L}_{\beta_{j} \phi}=\frac{\partial^{2} L_{p}(\boldsymbol{\theta})}{\partial \beta_{j} \partial \phi}=\frac{2}{\phi^{2}} \sum_{i=1}^{n}\left(\epsilon_{i}-\rho \epsilon_{i-1}\right)\left(x_{i j}-\rho x_{(i-1) j}\right)\left[W_{g}\left(u_{i}\right)+W_{g}^{\prime}\left(u_{i}\right) u_{i}\right] \quad \mathrm{e} \\
\ddot{L}_{\beta_{j} \rho}=\frac{\partial^{2} L_{p}(\boldsymbol{\theta})}{\partial \beta_{j} \partial \rho}=\frac{4}{\phi} \sum_{i=1}^{n}\left\{W_{g}^{\prime}\left(u_{i}\right) u_{i}\left(x_{i j}-\rho x_{(i-1) j}\right) \epsilon_{i-1}\right\}+ \\
+\frac{2}{\phi} \sum_{i=1}^{n}\left\{W_{g}\left(u_{i}\right)\left[\epsilon_{i-1}\left(x_{i j}-\rho x_{(i-1) j}\right)+x_{(i-1) j}\left(\epsilon_{i}-\rho \epsilon_{i-1}\right)\right]\right\} .
\end{gathered}
$$


É fácil ver que, em notação matricial, podemos escrever estas expressões da seguinte forma:

$$
\begin{gathered}
\ddot{\mathbf{L}}_{\beta \phi}=\frac{\partial^{2} L_{p}(\boldsymbol{\theta})}{\partial \boldsymbol{\beta} \partial \phi}=\frac{1}{\phi^{2}}\left[(\mathbf{A X})^{\top}(-\mathbf{D}(\mathbf{v})+2 \mathbf{D}(\mathbf{d})) \mathbf{A} \boldsymbol{\epsilon}\right] \quad \mathrm{e} \\
\ddot{\mathbf{L}}_{\beta \rho}=\frac{\partial^{2} L_{p}(\boldsymbol{\theta})}{\partial \beta \partial \rho}=\frac{1}{\phi}\left[(\mathbf{B} \boldsymbol{\epsilon})^{\top}(\mathbf{D}(\mathbf{v})-4 \mathbf{D}(\mathbf{d})) \mathbf{A X}+(\mathbf{B X})^{\top} \mathbf{D}(\mathbf{v}) \mathbf{A} \boldsymbol{\epsilon}\right] .
\end{gathered}
$$

Os cálculos dos elementos cruzados do parâmetro f em relação a $\phi$ e $\rho$ são similares aos mostrados acima. Assim, é fácil mostrar que

$$
\begin{gathered}
\ddot{\mathbf{L}}_{f \phi}=\frac{\partial^{2} L_{p}(\boldsymbol{\theta})}{\partial \mathbf{f} \partial \phi}=\frac{1}{\phi^{2}}\left[(\mathbf{A N})^{\top}(-\mathbf{D}(\mathbf{v})+2 \mathbf{D}(\mathbf{d})) \mathbf{A} \boldsymbol{\epsilon}\right] \quad \mathrm{e} \\
\ddot{\mathbf{L}}_{f \rho}=\frac{\partial^{2} L_{p}(\boldsymbol{\theta})}{\partial \mathbf{f} \partial \rho}=\frac{1}{\phi}\left[(\mathbf{B} \boldsymbol{\epsilon})^{\top}(\mathbf{D}(\mathbf{v})-4 \mathbf{D}(\mathbf{d})) \mathbf{A N}+(\mathbf{B N})^{\top} \mathbf{D}(\mathbf{v}) \mathbf{A} \boldsymbol{\epsilon}\right] .
\end{gathered}
$$

Por fim, para o elemento entre $\phi$ e $\rho$, encontramos

$$
\begin{array}{r}
\ddot{L}_{\rho \phi}=\frac{\partial^{2} L_{p}(\boldsymbol{\theta})}{\partial \rho \partial \phi}=\frac{2}{\phi^{2}} \sum_{i=1}^{n}\left(\epsilon_{i}-\right. \\
\left.=\rho \epsilon_{i-1}\right) \epsilon_{i-1}\left[W_{g}\left(u_{i}\right)+W_{g}^{\prime}\left(u_{i}\right) u_{i}\right]= \\
=\frac{1}{\phi^{2}}\left[(\mathbf{B} \boldsymbol{\epsilon})^{\top}(\mathbf{D}(\mathbf{v})-2 \mathbf{D}(\mathbf{d})) \mathbf{A} \boldsymbol{\epsilon}\right]
\end{array}
$$

\section{C.2 Informação de Fisher}

Para os cálculos dos elementos da matriz de Fisher, apresentados nesta seção, inicialmente lembramos de algumas propriedades. Sejam $f_{g}=E\left\{W^{2}\left(U^{2}\right) U^{4}\right\}$ e $d_{g}=E\left\{W^{2}\left(U^{2}\right) U^{2}\right\}$ com $U \sim S(0,1)$ da mesma forma que definimos nos Capítulos 2 e 3. Também, usaremos as condições de regularidade dadas por

$$
\begin{aligned}
& \text { 1.) } E\left[\mathbf{U}_{\theta}\right]=\mathbf{0} \mathrm{e} \\
& \text { 2.) }-E\left[\frac{\partial^{2} L_{p}(\boldsymbol{\theta})}{\partial \boldsymbol{\theta} \partial \boldsymbol{\theta}^{\top}}\right]=E\left[\left(\frac{\partial L_{p}(\boldsymbol{\theta})}{\partial \boldsymbol{\theta}}\right)^{2}\right] \text {. }
\end{aligned}
$$


Além disso, conforme descrito no Capítulo 3, temos que

$$
\begin{aligned}
E\left[\epsilon_{i}\right] & =0 \mathrm{e} \\
\operatorname{Var}\left(\epsilon_{i}\right) & =\phi \xi \frac{\left(1-\rho^{2 i}\right)}{\left(1-\rho^{2}\right)} .
\end{aligned}
$$

Com todas estas definições e propriedades, podemos prosseguir para o cálculo da matriz de informação de Fisher. Calculamos a informação de Fisher em relação a todos os parâmetros, começando com a informação referente ao parâmetro $\phi$.

Através da primeira condição de regularidade definida acima, temos que $E\left\{U_{\phi}(\boldsymbol{\theta})\right\}=0$. Portanto, é fácil ver que $E\left\{W_{g}\left(u_{i}\right) u_{i}\right\}=-\frac{1}{2}$, para qualquer $i$.

Para encontrarmos a informação para esse parâmetro, usamos a segunda condição de regularidade dada abaixo

$$
\begin{array}{r}
E\left[\left(\frac{\partial l_{i}(\boldsymbol{\theta})}{\partial \phi}\right)^{2}\right]=E\left[\frac{1}{4 \phi^{2}}+W_{g}^{2}\left(u_{i}\right) \frac{u_{i}^{2}}{\phi^{2}}+W_{g}\left(u_{i}\right) \frac{u_{i}}{\phi^{2}}\right]=\frac{1}{\phi^{2}} \\
{\left[\frac{1}{4}+f_{g}-\frac{1}{2}\right]=} \\
=\frac{1}{\phi^{2}}\left[f_{g}-\frac{1}{4}\right]
\end{array}
$$

Lembrando que

$$
L(\boldsymbol{\theta})=-\frac{n}{2} \log \phi+\sum_{i=1}^{n} \log \left\{g\left(u_{i}\right)\right\}=\sum_{i=1}^{n} l_{i}(\boldsymbol{\theta})
$$

em que,

$$
l_{i}(\boldsymbol{\theta})=-\frac{1}{2} \log \phi+\log \left\{g\left(u_{i}\right)\right\} .
$$

Logo, é possível definir a informação da seguinte forma:

$$
K_{\phi \phi}=\frac{n}{4 \phi^{2}}\left(4 f_{g}-1\right) \text {. }
$$

Prosseguimos agora para o cálculo da informação referente ao parâmetro $\boldsymbol{\beta}$ e ao parâmetro f. Como os cálculos são muito similares para os dois parâmetros, mostraremos passo a passo apenas para $\boldsymbol{\beta}$.

Aqui, mais uma vez, usaremos a segunda condição de regularidade. Temos que 


$$
\begin{array}{r}
{\left[\left(\frac{\partial l_{i}(\boldsymbol{\theta})}{\partial \beta_{j}}\right)^{2}\right]=\left[W_{g}\left(u_{i}\right) \frac{2}{\phi}\left(\epsilon_{i}-\rho \epsilon_{i-1}\right)\left(x_{i, j}-\rho x_{i-1, j}\right)\right]^{2}=} \\
=\frac{4}{\phi} W_{g}^{2}\left(u_{i}\right) u_{i}\left(x_{i, j}-\rho x_{i-1, j}\right)^{2} .
\end{array}
$$

Logo, podemos escrever $\mathbf{K}_{\beta \beta}$ na seguinte forma matricial:

$$
\mathbf{K}_{\beta \beta}=\frac{4}{\phi} d_{g}(\mathbf{A X})^{\top}(\mathbf{A X}),
$$

em que $\mathbf{X}$ e $\mathbf{A}$ foram definidas anteriormente.

Da mesma forma, temos que

$$
\mathbf{K}_{f f}=\frac{4}{\phi} d_{g}(\mathbf{A N})^{\top}(\mathbf{A N})-\alpha \mathbf{K} .
$$

O termo $\alpha \mathbf{K}$ aparece devido a estarmos utilizando o método de verossimilhança penalizada. A matriz $\mathbf{N}$ também foi definida nas seções anteriores.

Iremos agora encontrar a informação para o parâmetro $\rho$. Mais uma vez, por facilidade, usaremos a segunda condição de regularidade,

$$
\left[\left(\frac{\partial l_{i}(\boldsymbol{\theta})}{\partial \rho}\right)^{2}\right]=\left[W_{g}\left(u_{i}\right) \frac{2}{\phi}\left(\epsilon_{i}-\rho \epsilon_{i-1}\right) \epsilon_{i-1}\right]^{2}=\frac{4}{\phi} W_{g}^{2}\left(u_{i}\right) u_{i} \epsilon_{i-1}^{2} .
$$

Então,

$$
E\left[\frac{4}{\phi} W_{g}^{2}\left(u_{i}\right) u_{i} \epsilon_{i-1}^{2}\right]=E\left[E\left[\frac{4}{\phi} W_{g}^{2}\left(u_{i}\right) u_{i} \epsilon_{i-1}^{2} \mid y_{i-1}\right]\right]=E\left[\frac{4}{\phi} d_{g} \epsilon_{i-1}^{2}\right] .
$$

Utilizando-se dos momentos de $\epsilon_{i}$, podemos escrever:

$$
\begin{array}{r}
K_{\rho \rho}=\frac{4}{\phi} d_{g} \sum_{i=2}^{n} E\left(\epsilon_{i-1}^{2}\right)=\frac{4}{\phi} d_{g} \sum_{i=1}^{n-1} E\left(\epsilon_{i}^{2}\right)=4 d_{g} \xi \sum_{i=1}^{n-1} \frac{1-\rho^{2 i}}{1-\rho^{2}}= \\
=\frac{4 d_{g} \xi}{1-\rho^{2}}\left[(n-1)-\left(\frac{\rho^{2}\left(1-\rho^{2(n-1)}\right)}{1-\rho^{2}}\right)\right] .
\end{array}
$$


Continuaremos para as esperanças cruzadas. A principal estratégia utilizada para o cálculo destas esperanças será aplicar a segunda condição de regularidade com as informações já encontradas. Assim será possível encontrarmos alguns valores esperados de forma mais fácil. Sem essa estratégia, seria muito complicado obter tais esperanças.

Por exemplo, $\mathbf{K}_{\beta \beta}$ já foi encontrada sendo igual a $\frac{4}{\phi} d_{g}(\mathbf{A X})^{\top}(\mathbf{A X})$. Também, temos que,

$$
\mathbf{K}_{\beta \beta}=-E\left(\frac{1}{\phi}\left[\mathbf{A} \mathbf{X}^{\top}(-\mathbf{D}(\mathbf{v})+4 \mathbf{D}(\mathbf{d}))(\mathbf{A X})\right]\right) .
$$

Portanto, é fácil ver que $E\{-\mathbf{D}(\mathbf{v})+4 \mathbf{D}(\mathbf{d})\}=-4 d_{g}$. Assim, conseguimos encontrar a informação de Fisher cruzada entre $\boldsymbol{\beta}$ e f, dada por

$$
\mathbf{K}_{\beta f}=-E\left\{\frac{1}{\phi}\left[(\mathbf{A X})^{\top}(-\mathbf{D}(\mathbf{v})+4 \mathbf{D}(\mathbf{d})) \mathbf{A N}\right]\right\}=\frac{1}{\phi} 4 d_{g}\left[(\mathbf{A X})^{\top}(\mathbf{A N})\right]
$$

em que as matrizes $\mathbf{D}(\mathbf{v})$ e $\mathbf{D}(\mathbf{d})$ também já foram definidas anteriormente.

Temos, então, que os vetores de parâmetros $\boldsymbol{\beta}$ e f não são ortogonais.

Já, em anologia ao modelo simétrico tradicional, comentado no capítulo anterior, obtemos o seguinte:

$$
E\left[\left\{W_{g}\left(u_{i}\right)+W_{g}^{\prime}\left(u_{i}\right) u_{i}\right\}\left(\epsilon_{i}-\rho \epsilon_{i-1}\right) \mid y_{i-1}\right]=0 .
$$

Isto pode ser verificado, pois no modelo simétrico linear usual $\mathbf{K}_{\beta \phi}$ é igual a 0. Com esse resultado, conseguimos encontrar as seguintes esperanças cruzadas:

$$
\begin{aligned}
\mathbf{K}_{\phi \beta} & =-E\left[\frac{1}{\phi^{2}}\left\{(\mathbf{A X})^{\top}(-\mathbf{D}(\mathbf{v})+2 \mathbf{D}(\mathbf{d})) \mathbf{A} \boldsymbol{\epsilon}\right\}\right]=\mathbf{0} \\
\mathbf{K}_{\phi f} & =-E\left[\frac{1}{\phi^{2}}\left\{(\mathbf{A N})^{\top}(-\mathbf{D}(\mathbf{v})+2 \mathbf{D}(\mathbf{d})) \mathbf{A} \boldsymbol{\epsilon}\right\}\right]=\mathbf{0} \quad \mathrm{e} \\
K_{\phi \rho} & =-E\left[\frac{1}{\phi^{2}}\left\{(\mathbf{B} \boldsymbol{\epsilon})^{\top}(\mathbf{D}(\mathbf{v})-2 \mathbf{D}(\mathbf{d})) \mathbf{A} \boldsymbol{\epsilon}\right\}\right]=0 .
\end{aligned}
$$

Como $E\left[\mathbf{U}_{\beta}\right]=\mathbf{0}$, segue que

$$
E\left\{\frac{1}{\phi}(\mathbf{A X})^{\top} \mathbf{D}(\mathbf{v}) \mathbf{A} \boldsymbol{\epsilon}\right\}=\mathbf{0} \Leftrightarrow E[\mathbf{D}(\mathbf{v}) \mathbf{A} \boldsymbol{\epsilon}]=\mathbf{0} .
$$


Com isso,

$$
\begin{aligned}
\mathbf{K}_{\rho \beta}=-\frac{1}{\phi} E\left[(\mathbf{B} \boldsymbol{\epsilon})^{\top}(\mathbf{D}(\mathbf{v})\right. & \left.-4 \mathbf{D}(\mathbf{d})) \mathbf{A X}+(\mathbf{B X})^{\top} \mathbf{D}(\mathbf{v}) \mathbf{A} \boldsymbol{\epsilon}\right]= \\
& =-\frac{1}{\phi} E\left[(\mathbf{B} \boldsymbol{\epsilon})^{\top}(\mathbf{D}(\mathbf{v})-4 \mathbf{D}(\mathbf{d})) \mathbf{A X}\right] .
\end{aligned}
$$

Além disso, também mostramos que $E\{-\mathbf{D}(\mathbf{v})+4 \mathbf{D}(\mathbf{d})\}=-4 d_{g}$. Logo,

$$
\begin{array}{r}
\mathbf{K}_{\rho \beta}=-E\left(E\left[\frac{1}{\phi}\left\{(\mathbf{B} \boldsymbol{\epsilon})^{\top}(\mathbf{D}(\mathbf{v})-4 \mathbf{D}(\mathbf{d})) \mathbf{A X}\right\}\right] \mid y_{i-1}\right)= \\
=E\left(\frac{1}{\phi}\left[(\mathbf{B} \boldsymbol{\epsilon})^{\top} 4 d_{g} \mathbf{A X}\right]\right)=\mathbf{0 .}
\end{array}
$$

uma vez que $E\left[(\mathbf{B} \epsilon)^{\top}\right]=\mathbf{0}$.

Por fim, do mesmo modo, temos que $\mathbf{K}_{\rho f}=\mathbf{0}$. 
116 MATRIZ DE INFORMAÇÃO DE FISHER C. 2 


\section{Apêndice D}

\section{Diagnóstico}

\section{D.1 Matriz de alavancas}

Nesta seção, apresentamos os cálculos necessários para a obtenção da matriz de alavancas, definida como

$$
\mathbf{G L}(\hat{\boldsymbol{\theta}})=\left.\left[\mathbf{D}_{\theta}\left(-\ddot{\mathbf{L}}_{\theta \theta}\right)^{-1} \ddot{\mathbf{L}}_{\theta y}\right]\right|_{\boldsymbol{\theta}=\hat{\boldsymbol{\theta}}}
$$

A matriz $\mathbf{D}_{\theta}$ foi encontrada no Capítulo 4 , enquanto que a matriz $\ddot{\mathbf{L}}_{\theta \theta}$ foi definida no Capítulo 3. Portanto, temos que encontrar apenas $\ddot{\mathbf{L}}_{\theta y}$. Para facilitar a organização dos cálculos, escrevemos a matriz procurada da seguinte forma:

$$
\ddot{\mathbf{L}}_{\theta \mathbf{y}}=\left[\begin{array}{c}
\ddot{\mathbf{L}}_{\beta y} \\
\ddot{\mathbf{L}}_{f y} \\
\ddot{\mathbf{L}}_{\phi y} \\
\ddot{\mathbf{L}}_{\rho y}
\end{array}\right] .
$$

Antes de começarmos os cálculos da obtenção dessa matriz, iremos obter algumas derivadas iniciais que serão utilizadas adiante:

$$
\begin{gathered}
\\
\frac{\partial \epsilon_{i}}{\partial y_{k}}= \begin{cases}1, & \text { se } k=i \\
0, & \text { caso contrário, }\end{cases} \\
\frac{\partial W_{g}\left(u_{i}\right)}{\partial y_{k}}= \begin{cases}W_{g}^{\prime}\left(u_{i}\right) 2 \frac{\left(\epsilon_{i}-\rho \epsilon_{i-1}\right)}{\phi}, & \text { se } k=i \\
W_{g}^{\prime}\left(u_{i}\right) 2 \frac{\left(\epsilon_{i}-\rho \epsilon_{i-1}\right)}{\phi}(-\rho), & \text { se } k=i-1 \\
0, & \text { caso contrário. }\end{cases}
\end{gathered}
$$


Novamente pelo Capítulo 3, temos que

$$
U_{\beta_{j}}=\frac{\partial L_{p}(\theta)}{\partial \beta_{j}}=-\sum_{i=1}^{n} W_{g}\left(u_{i}\right) \frac{2}{\phi}\left(\epsilon_{i}-\rho \epsilon_{i-1}\right)\left(x_{i j}-\rho x_{(i-1) j}\right) .
$$

Assim,

$$
\begin{aligned}
\ddot{\mathbf{L}}_{\beta_{j} y_{k}} & =\frac{\partial U_{\beta_{j}}}{\partial y_{k}}= \\
& =-W_{g}^{\prime}\left(u_{k}\right) 4 \frac{\left(\epsilon_{k}-\rho \epsilon_{k-1}\right)^{2}}{\phi^{2}}\left(x_{k j}-\rho x_{(k-1) j}\right)-W_{g}\left(u_{k}\right) \frac{2}{\phi}\left(x_{k j}-\rho x_{(k-1) j}\right)+ \\
& +W_{g}^{\prime}\left(u_{k+1}\right) 4 \rho \frac{\left(\epsilon_{k+1}-\rho \epsilon_{k}\right)^{2}}{\phi^{2}}\left(x_{(k+1) j}-\rho x_{k j}\right)+W_{g}\left(u_{k+1}\right) \frac{2 \rho}{\phi}\left(x_{(k+1) j}-\rho x_{k j}\right)= \\
& =-W_{g}^{\prime}\left(u_{k}\right) \frac{4}{\phi} u_{k}\left(x_{k j}-\rho x_{(k-1) j}\right)-W_{g}\left(u_{k}\right) \frac{2}{\phi}\left(x_{k j}-\rho x_{(k-1) j}\right)+ \\
& +W_{g}^{\prime}\left(u_{k+1}\right) \frac{4 \rho}{\phi} u_{k+1}\left(x_{(k+1) j}-\rho x_{k j}\right)+W_{g}\left(u_{k+1}\right) \frac{2 \rho}{\phi}\left(x_{(k+1) j}-\rho x_{k j}\right) .
\end{aligned}
$$

O termo acima é referente apenas a uma casela da matriz $\ddot{\mathbf{L}}_{\beta y}$, a $j$-ésima linha e $k$-ésima coluna. Com essa equação, podemos escrever a matriz procurada, em forma matricial, como a seguir:

$$
\ddot{\mathbf{L}}_{\beta y}=-\frac{4}{\phi} \mathbf{D}(\mathbf{d}) \mathbf{A X}+\frac{1}{\phi} \mathbf{D}(\mathbf{v}) \mathbf{A X}-\mathbf{B}^{\top}\left[\frac{4 \rho}{\phi} \mathbf{D}(\mathbf{d}) \mathbf{A X}\right]+\mathbf{B}^{\top}\left[\frac{\rho}{\phi} \mathbf{D}(\mathbf{v}) \mathbf{A X}\right]
$$

em que as matrizes $\mathbf{A}, \mathbf{B}, \mathbf{D}(\mathbf{d}), \mathbf{D}(\mathbf{v})$ e $\mathbf{X}$ já foram definidas no Capítulo 3.

Os cálculos em relação ao parâmetro f são similares. Assim, encontramos que

$$
\ddot{\mathbf{L}}_{f y}=-\frac{4}{\phi} \mathbf{D}(\mathbf{d}) \mathbf{A N}+\frac{1}{\phi} \mathbf{D}(\mathbf{v}) \mathbf{A} \mathbf{N}-\mathbf{B}^{\top}\left[\frac{4 \rho}{\phi} \mathbf{D}(\mathbf{d}) \mathbf{A N}\right]+\mathbf{B}^{\top}\left[\frac{\rho}{\phi} \mathbf{D}(\mathbf{v}) \mathbf{A} \mathbf{N}\right],
$$

em que a matriz $\mathbf{N}$ também já foi definida no Capítulo 3.

Nos capítulos anteriores, já calculamos

$$
U_{\phi}=\frac{\partial L_{\mathrm{p}}(\boldsymbol{\theta})}{\partial \phi}=-\frac{n}{2 \phi}-\sum_{i=1}^{n} W_{g}\left(u_{i}\right) \frac{u_{i}}{\phi}
$$


Portanto, precisamos apenas derivar a fórmula acima em relação a y,

$$
\begin{aligned}
\ddot{\mathbf{L}}_{\phi y_{k}} & =\frac{\partial U_{\phi}}{\partial y_{k}}=-W_{g}^{\prime}\left(u_{k}\right) \frac{2 u_{k}}{\phi^{2}}\left(\epsilon_{k}-\rho \epsilon_{k-1}\right)-W_{g}\left(u_{k}\right) \frac{2}{\phi^{2}}\left(\epsilon_{k}-\rho \epsilon_{k-1}\right)+ \\
& +W_{g}^{\prime}\left(u_{k+1}\right) \frac{2 \rho u_{k+1}}{\phi^{2}}\left(\epsilon_{k+1}-\rho \epsilon_{k}\right)+W_{g}\left(u_{k+1}\right) \frac{2 \rho}{\phi^{2}}\left(\epsilon_{k+1}-\rho \epsilon_{k}\right) .
\end{aligned}
$$

Em notação matricial, obtemos

$$
\ddot{\mathbf{L}}_{\phi y}=-\frac{2}{\phi^{2}} \mathbf{D}(\mathbf{d}) \mathbf{A} \boldsymbol{\epsilon}+\frac{1}{\phi^{2}} \mathbf{D}(\mathbf{v}) \mathbf{A} \boldsymbol{\epsilon}-\mathbf{B}^{\top}\left[\frac{2 \rho}{\phi^{2}} \mathbf{D}(\mathbf{d}) \mathbf{A} \boldsymbol{\epsilon}\right]+\mathbf{B}^{\top}\left[\frac{\rho}{\phi^{2}} \mathbf{D}(\mathbf{v}) \mathbf{A} \boldsymbol{\epsilon}\right]
$$

Por fim, temos que encontrar o vetor $\ddot{\mathbf{L}}_{\rho y}$. Novamente, pelos cálculos do Apêndice B, é possível ver que

$$
U_{\rho}=\frac{\partial L_{\mathrm{p}}(\boldsymbol{\theta})}{\partial \rho}=-\sum_{i=1}^{n} W_{g}\left(u_{i}\right) \frac{2}{\phi}\left(\epsilon_{i}-\rho \epsilon_{i-1}\right) \epsilon_{i-1} .
$$

Então,

$$
\begin{aligned}
\ddot{\mathbf{L}}_{\rho y_{k}} & =\frac{\partial U_{\rho}}{\partial y_{k}}=-W_{g}^{\prime}\left(u_{k}\right) \frac{4 u_{k}}{\phi}\left(\epsilon_{k-1}\right)-W_{g}\left(u_{k}\right) \frac{2}{\phi} \epsilon_{k-1}+ \\
& +W_{g}^{\prime}\left(u_{k+1}\right) \frac{4 \rho u_{k+1}}{\phi}\left(\epsilon_{k}\right)-W_{g}\left(u_{k+1}\right) \frac{2}{\phi}\left[\epsilon_{k+1}-2 \rho \epsilon_{k}\right]
\end{aligned}
$$

Mais uma vez, podemos escrever em termos matriciais do seguinte modo:

$$
\begin{aligned}
& \ddot{\mathbf{L}}_{\rho y}=\frac{4}{\phi} \mathbf{D}(\mathbf{d}) \mathbf{B} \boldsymbol{\epsilon}-\frac{1}{\phi} \mathbf{D}(\mathbf{v}) \mathbf{B} \boldsymbol{\epsilon}+\mathbf{B}^{\top}\left[\frac{4 \rho}{\phi} \mathbf{D}(\mathbf{d}) \mathbf{B} \boldsymbol{\epsilon}\right]-\mathbf{B}^{\top}\left[\frac{1}{\phi} \mathbf{D}(\mathbf{v}) \mathbf{C} \boldsymbol{\epsilon}\right], \text { em que } \\
& \mathbf{C}=\left(\begin{array}{rrrrr}
1 & 0 & 0 & \ldots & 0 \\
-2 \rho & 1 & 0 & \ldots & 0 \\
\vdots & & \vdots & & \vdots \\
0 & \ldots & 0 & -2 \rho & 1
\end{array}\right) .
\end{aligned}
$$

Com esse último cálculo, temos todos os elementos da matriz de alavancas definidos. 


\section{D.2 Influência local}

Nesta seção apresentamos os cálculos da matriz $\boldsymbol{\Delta}$, definida no Capítulo 4 e utilizada para detectar possíveis mudanças importantes nas estimativas dos parâmetros sob pequenas perturbações no modelo ou nos dados. Encontramos tal matriz para três esquemas de perturbação que serão apresentados em três subseções separadas. Primeiramente, supomos que a variável resposta seja acrescida de um delta. Já no segundo esquema, supomos que uma das variáveis explicativas também seja acrescida de um delta. Por fim, no terceiro esquema, avaliamos uma ponderação dos casos. Chamamos cada esquema, respectivamente, de perturbação aditiva na resposta do modelo, perturbação aditiva em uma variável contínua do modelo e de ponderação de casos.

\section{D.2.1 Perturbação aditiva na resposta do modelo}

Consideramos, nesta subseção, uma perturbação aditiva na variável resposta do modelo. Assim, consideramos que $y_{i w}=y_{i}+w_{i}, \forall i=1, \ldots, n$. O objetivo é obter a matriz $\boldsymbol{\Delta}$, cujos elementos são dados por

$$
\Delta_{i j}=\frac{\partial^{2} L(\boldsymbol{\theta} \mid \mathbf{w})}{\partial \theta_{i} \partial w_{j}}
$$

avaliados em $\hat{\boldsymbol{\theta}}$ e $\mathbf{w}_{0}=(0, \ldots, 0)^{\top}$ vetor de não perturbação.

Portanto, é necessário derivar as funções escore em relação ao vetor w. Antes, porém, devemos definir as seguintes quantidades:

$$
\begin{aligned}
\epsilon_{w i} & =y_{i}+w_{i}-\mathbf{x}_{i}^{\top} \boldsymbol{\beta}-\boldsymbol{\eta}_{i}^{\top} \mathbf{f} \mathrm{e} \\
u_{w i} & =\frac{\left(\epsilon_{w i}-\rho \epsilon_{w i-1}\right)^{2}}{\phi} .
\end{aligned}
$$

Além dessas, utilizaremos

$$
\begin{gathered}
\frac{\partial \epsilon_{w i}}{\partial w_{k}}= \begin{cases}1, & \text { se } k=i \\
0, & \text { caso contrário }\end{cases} \\
\frac{\partial W_{g}\left(u_{w i}\right)}{\partial w_{k}}= \begin{cases}W_{g}^{\prime}\left(u_{w i}\right) \frac{2}{\phi}\left(\epsilon_{w i}-\rho \epsilon_{w(i-1)}\right), & \text { se } k=i \\
-W_{g}^{\prime}\left(u_{w i}\right) \frac{2 \rho}{\phi}\left(\epsilon_{w i}-\rho \epsilon_{w(i-1)}\right), & \text { se } k=i-1 \\
0, & \text { caso contrário. }\end{cases}
\end{gathered}
$$


Temos que a função escore, em relação ao parâmetro $\beta_{j}$ supondo essa perturbação, é dada por

$$
U_{\beta_{j}}=\frac{\partial L_{\mathrm{p}}(\boldsymbol{\theta})}{\partial \beta_{j}}=-\sum_{i=1}^{n} W_{g}\left(u_{w i}\right) \frac{2}{\phi}\left(\epsilon_{w i}-\rho \epsilon_{w i-1}\right)\left(x_{i j}-\rho x_{(i-1) j}\right) .
$$

Portanto, temos que

$$
\begin{aligned}
& \frac{\partial U_{\beta_{j}}}{\partial w_{k}}=-W_{g}^{\prime}\left(u_{w k}\right) \frac{4}{\phi} u_{w k}\left(x_{k j}-\rho x_{(k-1) j}\right)-W_{g}\left(u_{w k}\right) \frac{2}{\phi}\left(x_{k j}-\rho x_{(k-1) j}\right)+ \\
& +\quad W_{g}^{\prime}\left(u_{w(k+1)}\right) \frac{4 \rho}{\phi} u_{w(k+1)}\left(x_{(k+1) j}-\rho x_{k j}\right)+W_{g}\left(u_{w(k+1)}\right) \frac{2 \rho}{\phi}\left(x_{(k+1) j}-\rho x_{k j}\right) .
\end{aligned}
$$

Utilizando as matrizes definidas no Capítulo 3, podemos escrever a expressão acima da seguinte forma:

$$
\frac{\partial \mathbf{U}_{\beta}}{\partial \mathbf{w}}=-\frac{4}{\phi} \mathbf{D}\left(\mathbf{d}_{w}\right) \mathbf{A X}+\frac{2}{\phi} \mathbf{D}\left(\mathbf{v}_{w}\right) \mathbf{A X}-\mathbf{B}^{\top}\left[\frac{4 \rho}{\phi} \mathbf{D}\left(\mathbf{d}_{w}\right) \mathbf{A X}\right]+\mathbf{B}^{\top}\left[\frac{2 \rho}{\phi} \mathbf{D}\left(\mathbf{v}_{w}\right) \mathbf{A X}\right]
$$

em que as matrizes $\mathbf{D}\left(\mathbf{d}_{w}\right)$ e $\mathbf{D}\left(\mathbf{v}_{w}\right)$ são iguais às matrizes $\mathbf{D}(\mathbf{d})$ e $\mathbf{D}(\mathbf{v})$, apenas substituindo o vetor $\mathbf{u}$ pelo vetor perturbado $\mathbf{u}_{w}$ definido acima.

De forma similar, encontramos

$$
\frac{\partial \mathbf{U}_{f}}{\partial \mathbf{w}}=-\frac{4}{\phi} \mathbf{D}\left(\mathbf{d}_{w}\right) \mathbf{A N}+\frac{2}{\phi} \mathbf{D}\left(\mathbf{v}_{w}\right) \mathbf{A N}-\mathbf{B}^{\top}\left[\frac{4 \rho}{\phi} \mathbf{D}\left(\mathbf{d}_{w}\right) \mathbf{A N}\right]+\mathbf{B}^{\top}\left[\frac{2 \rho}{\phi} \mathbf{D}\left(\mathbf{v}_{w}\right) \mathbf{A N}\right]
$$

A função escore do parâmetro $\phi$, com esse esquema de perturbação, é dada por

$$
U_{\phi}=\frac{\partial L_{\mathrm{p}}(\boldsymbol{\theta})}{\partial \phi}=-\frac{n}{2 \phi}-\sum_{i=1}^{n} W_{g}\left(u_{w i}\right) \frac{u_{w i}}{\phi} .
$$

Calculando a derivada dessa função para o vetor w, encontramos 


$$
\begin{aligned}
& \frac{\partial U_{\phi}}{\partial w_{k}}=-W_{g}^{\prime}\left(u_{w k}\right) \frac{2}{\phi^{2}} u_{w k}\left(\epsilon_{w k}-\rho \epsilon_{w(k-1)}\right)-W_{g}\left(u_{w k}\right) \frac{2}{\phi^{2}}\left(\epsilon_{w k}-\rho \epsilon_{w(k-1)}\right)+ \\
& +\quad W_{g}^{\prime}\left(u_{w(k+1)}\right) \frac{2 \rho}{\phi^{2}} u_{w(k+1)}\left(\epsilon_{w(k+1)}-\rho \epsilon_{w k)}\right)+W_{g}\left(u_{w(k+1)}\right) \frac{2 \rho}{\phi^{2}}\left(\epsilon_{w(k+1)}-\rho \epsilon_{w k}\right) .
\end{aligned}
$$

Em notação matricial, podemos escrever da seguinte forma:

$$
\frac{\partial U_{\phi}}{\partial \mathbf{w}}=-\frac{2}{\phi^{2}} \mathbf{D}\left(\mathbf{d}_{w}\right) \mathbf{A} \boldsymbol{\epsilon}+\frac{1}{\phi^{2}} \mathbf{D}\left(\mathbf{v}_{w}\right) \mathbf{A} \boldsymbol{\epsilon}-\mathbf{B}^{\top}\left[\frac{2 \rho}{\phi^{2}} \mathbf{D}\left(\mathbf{d}_{w}\right) \mathbf{A} \boldsymbol{\epsilon}\right]+\mathbf{B}^{\top}\left[\frac{\rho}{\phi^{2}} \mathbf{D}\left(\mathbf{v}_{w}\right) \mathbf{A} \boldsymbol{\epsilon}\right]
$$

Por fim, precisamos encontrar a função referente ao parâmetro $\rho$. Temos a escore nesse caso dado por

$$
U_{\rho}=\frac{\partial L_{\mathbf{p}}(\boldsymbol{\theta})}{\partial \rho}=-\sum_{i=1}^{n} W_{g}\left(u_{w i}\right) \frac{2}{\phi}\left(\epsilon_{w i}-\rho \epsilon_{w(i-1)}\right) \epsilon_{w(i-1)} .
$$

A derivada, então, é dada por

$$
\begin{aligned}
& \frac{\partial U_{\rho}}{\partial w_{k}}=-W_{g}^{\prime}\left(u_{w k}\right) \frac{4}{\phi} u_{w k}\left(\epsilon_{w(k-1)}\right)-W_{g}\left(u_{w k}\right) \frac{2}{\phi}\left(\epsilon_{w(k-1)}\right)+ \\
& +\quad W_{g}^{\prime}\left(u_{w(k+1)}\right) \frac{4 \rho}{\phi} u_{w(k+1)}\left(\epsilon_{w k}\right)-W_{g}\left(u_{w(k+1)}\right) \frac{2 \rho}{\phi}\left(\epsilon_{w(k+1)}-2 \rho \epsilon_{w k}\right),
\end{aligned}
$$

que pode ser escrita como

$$
\frac{\partial U_{\rho}}{\partial \mathbf{w}}=-\frac{4}{\phi} \mathbf{D}\left(\mathbf{d}_{w}\right) \mathbf{B} \boldsymbol{\epsilon}+\frac{1}{\phi} \mathbf{D}\left(\mathbf{v}_{w}\right) \mathbf{B} \boldsymbol{\epsilon}-\mathbf{B}^{\top}\left[\frac{4 \rho}{\phi} \mathbf{D}\left(\mathbf{d}_{w}\right) \mathbf{B} \boldsymbol{\epsilon}\right]-\mathbf{B}^{\top}\left[\frac{\rho}{\phi} \mathbf{D}\left(\mathbf{v}_{w}\right) \mathbf{C} \boldsymbol{\epsilon}\right] .
$$

Com esse último cálculo, completamos a matriz $\boldsymbol{\Delta}$ para esse primeiro esquema de perturbação, tornando-se possível encontrar a curvatura normal.

Lembramos que essas matrizes devem ser avaliadas em $\hat{\boldsymbol{\theta}}$ e no vetor de não perturbação $\left(\mathbf{w}_{0}\right)$, que nesse caso, é dado por $(0, \ldots, 0)^{\top}$. Assim, temos que

$$
\begin{aligned}
\hat{\epsilon}_{i} & =y_{i}-\mathbf{x}_{i}^{\top} \hat{\boldsymbol{\beta}}-\boldsymbol{\eta}_{i}^{\top} \hat{\mathbf{f}} \mathrm{e} \\
\hat{u}_{i} & =\frac{\left(\hat{\epsilon}_{i}-\rho \hat{\epsilon}_{i-1}\right)^{2}}{\phi} .
\end{aligned}
$$


Além disso, devemos avaliar as matrizes $\mathbf{D}\left(\mathbf{d}_{w}\right)$ e $\mathbf{D}\left(\mathbf{v}_{w}\right)$ em função de $\hat{\mathbf{u}}$. Denotamos essas matrizes diagonal por $\mathbf{D}(\hat{\mathbf{d}})$ e $\mathbf{D}(\hat{\mathbf{v}})$. Ainda, como $\mathbf{A}$ e $\mathbf{C}$ são funções de $\rho$, chamaremos de $\hat{\mathbf{A}}$ e $\hat{\mathbf{C}}$ essas matrizes utilizando $\hat{\rho}$. Assim, podemos escrever os elementos de $\boldsymbol{\Delta}$, avaliados em $\hat{\boldsymbol{\theta}}$ e $\mathbf{w}_{0}$, como

$$
\begin{aligned}
\frac{\partial \mathbf{U}_{\beta}}{\partial \mathbf{w}} & =-\frac{4}{\hat{\phi}} \mathbf{D}(\hat{\mathbf{d}}) \hat{\mathbf{A}} \mathbf{X}+\frac{2}{\hat{\phi}} \mathbf{D}(\hat{\mathbf{v}}) \hat{\mathbf{A}} \mathbf{X}-\mathbf{B}^{\top}\left[\frac{4 \hat{\rho}}{\hat{\phi}} \mathbf{D}(\hat{\mathbf{d}}) \hat{\mathbf{A}} \mathbf{X}\right]+\mathbf{B}^{\top}\left[\frac{2 \hat{\rho}}{\hat{\phi}} \mathbf{D}(\hat{\mathbf{v}}) \hat{\mathbf{A}} \mathbf{X}\right] \\
\frac{\partial \mathbf{U}_{f}}{\partial \mathbf{w}} & =-\frac{4}{\hat{\phi}} \mathbf{D}(\hat{\mathbf{d}}) \hat{\mathbf{A}} \mathbf{N}+\frac{2}{\hat{\phi}} \mathbf{D}(\hat{\mathbf{v}}) \hat{\mathbf{A}} \mathbf{N}-\mathbf{B}^{\top}\left[\frac{4 \hat{\rho}}{\hat{\phi}} \mathbf{D}(\hat{\mathbf{d}}) \hat{\mathbf{A}} \mathbf{N}\right]+\mathbf{B}^{\top}\left[\frac{2 \hat{\rho}}{\hat{\phi}} \mathbf{D}(\hat{\mathbf{v}}) \hat{\mathbf{A}} \mathbf{N}\right] \\
\frac{\partial U_{\phi}}{\partial \mathbf{w}} & =-\frac{2}{\hat{\phi}^{2}} \mathbf{D}(\hat{\mathbf{d}}) \hat{\mathbf{A}} \hat{\boldsymbol{\epsilon}}+\frac{1}{\hat{\phi}^{2}} \mathbf{D}(\hat{\mathbf{v}}) \hat{\mathbf{A}} \hat{\boldsymbol{\epsilon}}-\mathbf{B}^{\top}\left[\frac{2 \hat{\rho}}{\hat{\phi^{2}}} \mathbf{D}(\hat{\mathbf{d}}) \hat{\mathbf{A}} \hat{\boldsymbol{\epsilon}}\right]+\mathbf{B}^{\top}\left[\frac{\hat{\rho}}{\hat{\phi}^{2}} \mathbf{D}(\hat{\mathbf{v}}) \hat{\mathbf{A}} \hat{\boldsymbol{\epsilon}}\right] \mathrm{e} \\
\frac{\partial U_{\rho}}{\partial \mathbf{w}} & =-\frac{4}{\hat{\phi}} \mathbf{D}(\hat{\mathbf{d}}) \mathbf{B} \hat{\boldsymbol{\epsilon}}+\frac{1}{\hat{\phi}} \mathbf{D}(\hat{\mathbf{v}}) \mathbf{B} \hat{\boldsymbol{\epsilon}}-\mathbf{B}^{\top}\left[\frac{4 \hat{\rho}}{\hat{\phi}} \mathbf{D}(\hat{\mathbf{d}}) \mathbf{B} \hat{\boldsymbol{\epsilon}}\right]-\mathbf{B}^{\top}\left[\frac{\hat{\rho}}{\hat{\phi}} \mathbf{D}(\hat{\mathbf{v}}) \hat{\mathbf{C}} \hat{\boldsymbol{\epsilon}}\right]
\end{aligned}
$$

\section{D.2.2 Perturbação aditiva em uma variável contínua do modelo}

Nesta subseção, consideramos uma perturbação aditiva em uma variável contínua do modelo ao invés da perturbação ocorrer na variável resposta. Assim, supomos que a $r$-ésima variável explicativa fique expressa por $x_{w i}^{(r)}=$ $x_{i}^{(r)}+w_{i}, \forall i=1, \ldots, n$. Para este caso, temos as seguintes quantidades:

$$
\begin{aligned}
\epsilon_{w i} & =y_{i}-\mathbf{x}_{i}^{\top} \boldsymbol{\beta}-\boldsymbol{\eta}_{i}^{\top} \mathbf{f}-w_{i} \beta_{r} \mathrm{e} \\
u_{w i} & =\frac{\left(\epsilon_{w i}-\rho \epsilon_{w i-1}\right)^{2}}{\phi}
\end{aligned}
$$

Logo, obtemos as seguintes derivadas básicas:

$$
\begin{gathered}
\frac{\partial \epsilon_{w i}}{\partial w_{k}}= \begin{cases}-\beta_{r}, & \text { se } k=i \\
0, & \text { caso contrário }\end{cases} \\
\frac{\partial W_{g}\left(u_{w i}\right)}{\partial w_{k}}= \begin{cases}-W_{g}^{\prime}\left(u_{w i}\right) \frac{2 \beta_{r}}{\phi}\left(\epsilon_{w i}-\rho \epsilon_{w(i-1)}\right), & \text { se } k=i \\
W_{g}^{\prime}\left(u_{w i}\right) \frac{2 \rho \beta_{r}}{\phi}\left(\epsilon_{w i}-\rho \epsilon_{w(i-1)}\right), & \text { se } k=i-1 \\
0, & \text { caso contrário. }\end{cases}
\end{gathered}
$$

Com essas quantidades, os cálculos se tornam mais fáceis. Para os parâme- 
tros $\boldsymbol{\beta}$, temos que

$$
\begin{aligned}
& \frac{\partial U_{\beta_{j}}}{\partial w_{k}}=W_{g}^{\prime}\left(u_{w k}\right) \frac{4 \beta_{r}}{\phi} u_{w k}\left(x_{w k j}-\rho x_{w(k-1) j}\right)+W_{g}\left(u_{w k}\right) \frac{2 \beta_{r}}{\phi}\left(x_{w k j}-\rho x_{w(k-1) j}\right)- \\
& -\quad W_{g}^{\prime}\left(u_{w(k+1)}\right) \frac{4 \rho \beta_{r}}{\phi} u_{w(k+1)}\left(x_{w(k+1) j}-\rho x_{w k j}\right)-W_{g}\left(u_{w(k+1)}\right) \frac{2 \rho \beta_{r}}{\phi}\left(x_{w(k+1) j}-\rho x_{w k j}\right) .
\end{aligned}
$$

Logo, em notação matricial, obtemos

$$
\frac{\partial \mathbf{U}_{\beta}}{\partial \mathbf{w}}=\frac{4 \beta_{r}}{\phi} \mathbf{D}\left(\mathbf{d}_{w}\right) \mathbf{A} \mathbf{X}-\frac{\beta_{r}}{\phi} \mathbf{D}\left(\mathbf{v}_{w}\right) \mathbf{A X}+\mathbf{B}^{\top}\left[\frac{4 \rho \beta_{r}}{\phi} \mathbf{D}\left(\mathbf{d}_{w}\right) \mathbf{A X}\right]-\mathbf{B}^{\top}\left[\frac{\rho \beta_{r}}{\phi} \mathbf{D}\left(\mathbf{v}_{w}\right) \mathbf{A X}\right]
$$

Equivalentemente, para o parâmetro f, escrevemos da seguinte maneira:

$$
\frac{\partial \mathbf{U}_{f}}{\partial \mathbf{w}}=\frac{4 \beta_{r}}{\phi} \mathbf{D}\left(\mathbf{d}_{w}\right) \mathbf{A N}-\frac{\beta_{r}}{\phi} \mathbf{D}\left(\mathbf{v}_{w}\right) \mathbf{A} \mathbf{N}+\mathbf{B}^{\top}\left[\frac{4 \rho \beta_{r}}{\phi} \mathbf{D}\left(\mathbf{d}_{w}\right) \mathbf{A N}\right]-\mathbf{B}^{\top}\left[\frac{\rho \beta_{r}}{\phi} \mathbf{D}\left(\mathbf{v}_{w}\right) \mathbf{A N}\right]
$$

Para os demais parâmetros, assim como foi para $\boldsymbol{\beta}$, os cálculos são similares aos obtidos no primeiro esquema de perturbação. Para $\phi$, encontramos

$$
\begin{aligned}
& \frac{\partial U_{\phi}}{\partial w_{k}}=W_{g}^{\prime}\left(u_{w k}\right) \frac{2 \beta_{r}}{\phi^{2}} u_{w k}\left(\epsilon_{w k}-\rho \epsilon_{w(k-1)}\right)+W_{g}\left(u_{w k}\right) \frac{2 \beta_{r}}{\phi^{2}}\left(\epsilon_{w k}-\rho \epsilon_{w(k-1)}\right)- \\
& -\quad W_{g}^{\prime}\left(u_{w(k+1)}\right) \frac{2 \rho \beta_{r}}{\phi^{2}} u_{w(k+1)}\left(\epsilon_{w(k+1)}-\rho \epsilon_{w k)}\right)-W_{g}\left(u_{w(k+1)}\right) \frac{2 \rho \beta_{r}}{\phi^{2}}\left(\epsilon_{w(k+1)}-\rho \epsilon_{w k}\right),
\end{aligned}
$$

que pode ser representado por

$$
\frac{\partial U_{\phi}}{\partial \mathbf{w}}=\frac{2 \beta_{r}}{\phi^{2}} \mathbf{D}\left(\mathbf{d}_{w}\right) \mathbf{A} \boldsymbol{\epsilon}-\frac{\beta_{r}}{\phi^{2}} \mathbf{D}\left(\mathbf{v}_{w}\right) \mathbf{A} \boldsymbol{\epsilon}+\mathbf{B}^{\top}\left[\frac{2 \rho \beta_{r}}{\phi^{2}} \mathbf{D}\left(\mathbf{d}_{w}\right) \mathbf{A} \boldsymbol{\epsilon}\right]-\mathbf{B}^{\top}\left[\frac{\rho \beta_{r}}{\phi^{2}} \mathbf{D}\left(\mathbf{v}_{w}\right) \mathbf{A} \boldsymbol{\epsilon}\right] .
$$

Por fim, para o parâmetro $\rho$, obtemos

$$
\begin{aligned}
& \frac{\partial U_{\rho}}{\partial w_{k}}=W_{g}^{\prime}\left(u_{w k}\right) \frac{4 \beta_{r}}{\phi} u_{w k}\left(\epsilon_{w(k-1)}\right)+W_{g}\left(u_{w k}\right) \frac{2 \beta_{r}}{\phi}\left(\epsilon_{w(k-1)}\right)- \\
& -\quad W_{g}^{\prime}\left(u_{w(k+1)}\right) \frac{4 \rho \beta_{r}}{\phi} u_{w(k+1)}\left(\epsilon_{w k}\right)+W_{g}\left(u_{w(k+1)}\right) \frac{2 \rho \beta_{r}}{\phi}\left(\epsilon_{w(k+1)}-2 \rho \epsilon_{w k}\right) .
\end{aligned}
$$


Esse termo, escrito em forma matricial, fica dado por

$\frac{\partial U_{\rho}}{\partial \mathbf{w}}=\frac{4 \beta_{r}}{\phi} \mathbf{D}\left(\mathbf{d}_{w}\right) \mathbf{B} \boldsymbol{\epsilon}-\frac{\beta_{r}}{\phi} \mathbf{D}\left(\mathbf{v}_{w}\right) \mathbf{B} \boldsymbol{\epsilon}+\mathbf{B}^{\top}\left[\frac{4 \rho \beta_{r}}{\phi} \mathbf{D}\left(\mathbf{d}_{w}\right) \mathbf{B} \boldsymbol{\epsilon}\right]+\mathbf{B}^{\top}\left[\frac{\rho \beta_{r}}{\phi} \mathbf{D}\left(\mathbf{v}_{w}\right) \mathbf{C} \boldsymbol{\epsilon}\right]$.

Alcançamos, assim, a matriz necessária para a obtenção da matriz de cuvartura normal para este esquema de perturbação.

Avaliando este esquema em $\hat{\boldsymbol{\theta}}$ e $\mathbf{w}_{0}$, verificamos que os vetores $\hat{\boldsymbol{\epsilon}}$ e $\hat{\mathbf{u}}$ e as matrizes $\mathbf{D}(\hat{\mathbf{d}})$ e $\mathbf{D}(\hat{\mathbf{v}})$ serão iguais aos elementos obtidos com a perturbação anterior (perturbação aditiva na variável resposta). Portanto, podemos escrever os elementos de $\boldsymbol{\Delta}$ como

$$
\begin{aligned}
\frac{\partial \mathbf{U}_{\beta}}{\partial \mathbf{w}} & =-\frac{4 \hat{\beta}_{r}}{\hat{\phi}} \mathbf{D}(\hat{\mathbf{d}}) \hat{\mathbf{A}} \mathbf{X}+\frac{\hat{\beta_{r}}}{\hat{\phi}} \mathbf{D}(\hat{\mathbf{v}}) \hat{\mathbf{A}} \mathbf{X}+\mathbf{B}^{\top}\left[\frac{4 \hat{\rho} \hat{\beta}_{r}}{\hat{\phi}} \mathbf{D}(\hat{\mathbf{d}}) \hat{\mathbf{A}} \mathbf{X}\right]-\mathbf{B}^{\top}\left[\frac{\hat{\rho} \hat{\beta}_{r}}{\hat{\phi}} \mathbf{D}(\hat{\mathbf{v}}) \hat{\mathbf{A}} \mathbf{X}\right], \\
\frac{\partial \mathbf{U}_{f}}{\partial \mathbf{w}} & =-\frac{4 \hat{\beta}_{r}}{\hat{\phi}} \mathbf{D}(\hat{\mathbf{d}}) \hat{\mathbf{A}} \mathbf{N}+\frac{\hat{\beta}_{r}}{\hat{\phi}} \mathbf{D}(\hat{\mathbf{v}}) \hat{\mathbf{A}} \mathbf{N}-\mathbf{B}^{\top}\left[\frac{4 \hat{\rho} \hat{\beta}_{r}}{\hat{\phi}} \mathbf{D}(\hat{\mathbf{d}}) \hat{\mathbf{A}} \mathbf{N}\right]+\mathbf{B}^{\top}\left[\frac{\hat{\rho} \hat{\beta}_{r}}{\hat{\phi}} \mathbf{D}(\hat{\mathbf{v}}) \hat{\mathbf{A}} \mathbf{N}\right], \\
\frac{\partial U_{\phi}}{\partial \mathbf{w}} & =-\frac{2 \hat{\beta}_{r}}{\hat{\phi}^{2}} \mathbf{D}(\hat{\mathbf{d}}) \hat{\mathbf{A}} \hat{\boldsymbol{\epsilon}}+\frac{\hat{\beta}_{r}}{\hat{\phi}^{2}} \mathbf{D}(\hat{\mathbf{v}}) \hat{\mathbf{A}} \hat{\boldsymbol{\epsilon}}-\mathbf{B}^{\top}\left[\frac{2 \hat{\rho} \hat{\beta}_{r}}{\hat{\phi}^{2}} \mathbf{D}(\hat{\mathbf{d}}) \hat{\mathbf{A}} \hat{\boldsymbol{\epsilon}}\right]+\mathbf{B}^{\top}\left[\frac{\hat{\rho} \hat{\beta}_{r}}{\hat{\phi}^{2}} \mathbf{D}(\hat{\mathbf{v}}) \hat{\mathbf{A}} \hat{\boldsymbol{\epsilon}}\right] \mathrm{e} \\
\frac{\partial U_{\rho}}{\partial \mathbf{w}} & =-\frac{4 \hat{\beta}_{r}}{\hat{\phi}} \mathbf{D}(\hat{\mathbf{d}}) \mathbf{B} \hat{\boldsymbol{\epsilon}}+\frac{\hat{\beta}_{r}}{\hat{\phi}} \mathbf{D}(\hat{\mathbf{v}}) \mathbf{B} \hat{\boldsymbol{\epsilon}}-\mathbf{B}^{\top}\left[\frac{4 \hat{\rho} \hat{\beta}_{r}}{\hat{\phi}} \mathbf{D}(\hat{\mathbf{d}}) \mathbf{B} \hat{\boldsymbol{\epsilon}}\right]-\mathbf{B}^{\top}\left[\frac{\hat{\rho} \hat{\beta}_{r}}{\hat{\phi}} \mathbf{D}(\hat{\mathbf{v}}) \hat{\mathbf{C}} \hat{\boldsymbol{\epsilon}}\right] .
\end{aligned}
$$

\section{D.2.3 Ponderação de casos}

Agora, consideramos que cada observação possui um peso diferente no processo de estimação, assim, iremos supor que o logaritmo da verossimilhança seja dado por

$$
L_{p}(\boldsymbol{\theta} \mid \mathbf{w})=\sum_{1}^{n} w_{i} L_{i}(\boldsymbol{\theta})-\frac{\alpha}{2} \mathbf{f}^{\top} \mathbf{K} \mathbf{f},
$$

em que $L_{i}(\boldsymbol{\theta})=-\frac{1}{2} \log \phi+\log \left\{g\left(u_{i}\right)\right\}$ e $\mathbf{w}=\left(w_{1}, \ldots, w_{n}\right)^{\top}$ é o vetor de pesos, com $0 \leq w_{i} \leq 1$. Para este esquema de perturbação, o vetor de não perturbação é $\mathbf{w}_{0}=(1, \ldots, 1)^{\top}$

Facilmente, podemos observar que, para este esquema de perturbação, as funções escores são dadas por: 


$$
\begin{aligned}
& U_{\beta_{j}}=\frac{\partial L_{\mathrm{p}}(\boldsymbol{\theta} \mid \mathbf{w})}{\partial \beta_{j}}=-\sum_{i=1}^{n} w_{i} W_{g}\left(u_{i}\right) \frac{2}{\phi}\left(\epsilon_{i}-\rho \epsilon_{i-1}\right)\left(x_{i j}-\rho x_{(i-1) j}\right), \\
&(j=1, \ldots, p), \\
& U_{f_{j}}= \frac{\partial L_{\mathrm{p}}(\boldsymbol{\theta} \mid \mathbf{w})}{\partial f_{j}}=-\sum_{i=1}^{n} w_{i}\left\{W_{g}\left(u_{i}\right) \frac{2}{\phi}\left(\epsilon_{i}-\rho \epsilon_{i-1}\right)\left(\eta_{i j}-\rho \eta_{(i-1) j}\right)\right\}-[\alpha \mathbf{K} \mathbf{f}]_{j}, \\
&(j=1, \ldots, q), \\
& U_{\phi}=\frac{\partial L_{\mathrm{p}}(\boldsymbol{\theta} \mid \mathbf{w})}{\partial \phi}=\sum_{i=1}^{n} w_{i}\left\{-\frac{1}{2 \phi}-W_{g}\left(u_{i}\right) \frac{u_{i}}{\phi}\right\} \mathrm{e} \\
& U_{\rho}=\frac{\partial L_{\mathrm{p}}(\boldsymbol{\theta} \mid \mathbf{w})}{\partial \rho}=-\sum_{i=1}^{n} w_{i} W_{g}\left(u_{i}\right) \frac{2}{\phi}\left(\epsilon_{i}-\rho \epsilon_{i-1}\right) \epsilon_{i-1} .
\end{aligned}
$$

Podemos, com os termos definidos acima, derivarmos em relação a w e obtermos a matriz de curvatura normal. Assim,

$$
\begin{aligned}
\frac{\partial U_{\beta_{j}}}{\partial w_{i}} & =-W_{g}\left(u_{i}\right) \frac{2}{\phi}\left(\epsilon_{i}-\rho \epsilon_{i-1}\right)\left(x_{i j}-\rho x_{(i-1) j}\right) \\
\frac{\partial U_{f_{j}}}{\partial w_{i}} & =-W_{g}\left(u_{i}\right) \frac{2}{\phi}\left(\epsilon_{i}-\rho \epsilon_{i-1}\right)\left(\eta_{i j}-\rho \eta_{(i-1) j}\right) \\
\frac{\partial U_{\phi}}{\partial w_{i}} & =-\frac{1}{2 \phi}-W_{g}\left(u_{i}\right) \frac{u_{i}}{\phi} \mathrm{e} \\
\frac{\partial U_{\rho}}{\partial w_{i}} & =-W_{g}\left(u_{i}\right) \frac{2}{\phi}\left(\epsilon_{i}-\rho \epsilon_{i-1}\right) \epsilon_{i-1} .
\end{aligned}
$$

Em notação matricial e avaliando os elementos acima em $\hat{\boldsymbol{\theta}}$ e $\mathbf{w}_{0}$, temos que os elementos da matriz $\boldsymbol{\Delta}$ são escritos como: 
D.2

$$
\begin{aligned}
\frac{\partial \mathbf{U}_{\beta}}{\partial \mathbf{w}} & =(\hat{\mathbf{A}} \mathbf{X})^{\top} \frac{\mathbf{D}(\hat{\mathbf{v}})}{\hat{\phi}} \mathbf{D}\left(\hat{\mathbf{A}}_{\epsilon}\right), \\
\frac{\partial \mathbf{U}_{f}}{\partial \mathbf{w}} & =(\hat{\mathbf{A}} \mathbf{N})^{\top} \frac{\mathbf{D}(\hat{\mathbf{v}})}{\hat{\phi}} \mathbf{D}\left(\hat{\mathbf{A}}_{\epsilon}\right), \\
\frac{\partial U_{\phi}}{\partial \mathbf{w}} & =-\frac{1}{2 \hat{\phi}} \mathbf{1}_{n}-\frac{\mathbf{D}(\hat{\mathbf{d}})}{\hat{\phi}} \mathbf{1}_{n} \mathrm{e} \\
\frac{\partial U_{\rho}}{\partial \mathbf{w}} & =-\left((\mathbf{B} \hat{\boldsymbol{\epsilon}})^{\top} \frac{\mathbf{D}(\hat{\mathbf{v}})}{\hat{\phi}} \mathbf{D}\left(\hat{\mathbf{A}}_{\epsilon}\right)\right)^{\top},
\end{aligned}
$$

em que $\mathbf{1}_{n}=(1, \ldots, 1)^{\top}$ e $\left(\mathbf{D}\left(\hat{\mathbf{A}}_{\epsilon}\right)\right)$ é uma matriz diagonal com elementos dados por $\hat{\mathbf{A}} \hat{\boldsymbol{\epsilon}}$.

Assim, terminamos os cálculos da matriz de curvatura normal para o esquema de ponderação de casos. 


\section{Apêndice E}

\section{Aplicações}

Tabela E.1: Estimativas dos parâmetros do modelo linear com erros autoregressivos $A R(1)$ ajustado aos dados sobre temperaturas.

\begin{tabular}{|r|r|r|r|r|}
\hline Efeito & Estimativa & Erro Padrão & Estatística t & Valor-p \\
\hline Intercepto & 5,049 & 1,006 & 5,017 & $<0,001$ \\
Cananéia & 0,801 & 0,044 & 18,040 & $<0,001$ \\
$\sigma^{2}$ & 0,659 & 0,013 & 50,692 & $<0,001$ \\
$\rho$ & 0,7679 & 0,058 & 13,263 & $<0,001$ \\
\hline
\end{tabular}

Tabela E.2: Estimativas dos parâmetros do modelo parcialmente linear normal com erros autoregressivos AR(1) ajustado aos dados de temperaturas.

\begin{tabular}{|r|r|r|r|r|}
\hline Efeito & Estimativa & Erro Padrão & Estatística t & Valor-p \\
\hline Intercepto & 4,645 & 0,075 & 61,913 & $<0,001$ \\
Cananéia & 0,825 & 0,003 & 239,65 & $<0,001$ \\
$\sigma^{2}$ & 0,568 & 0,007 & 84,853 & $<0,001$ \\
$\rho$ & 0,551 & 0,008 & 65,752 & $<0,001$ \\
\hline
\end{tabular}


Tabela E.3: Estimativas dos parâmetros do modelo parcialmente linear $t$ de Student (3 graus de liberdade) com erros autoregressivos AR(1) ajustado aos dados de temperaturas.

\begin{tabular}{|r|r|r|r|r|}
\hline Efeito & Estimativa & Erro Padrão & Estatística t & Valor-p \\
\hline Intercepto & 5,174 & 0,056 & 91,769 & $<0,001$ \\
Cananéia & 0,807 & 0,003 & 310,369 & $<0,001$ \\
$\sigma^{2}$ & 0,717 & 0,01 & 69,993 & $<0,001$ \\
$\rho$ & 0,469 & 0,004 & 60,000 & $<0,001$ \\
\hline
\end{tabular}




\section{Referências Bibliográficas}

Akaike(1974) H. Akaike. A new look at the statistical model identification. Automatic Control, IEEE Transactions on, 19(6):716-723. Citado na pág.

Arellano-Valle(1994) R.B. Arellano-Valle. Distribuições Elipticas: Propriedades e Aplicações no Modelo de Regressão. Tese de Doutorado, Departamento de Estatística, Universidade de São Paulo. Citado na pág.

Atkinson(1985) A.C. Atkinson. Plots, Transformations, and Regression: an Introduction to Graphical Methods of Diagnostic Regression Analysis. Clarendon Press. Citado na pág.

Aydin(2011) Dursun Aydin. Partially linear models based on smoothing spline estimated by different selection methods: A simulation study. Inter $S$ tat. Citado na pág. 46

Berhane e Tibshirani(1998) K. Berhane e R.J. Tibshirani. Generalized additive models for longitudinal data. Canadian Journal of Statistics, 26 (4):517-535. Citado na pág.

Buja et al.(1989) A. Buja, T. Hastie e R. Tibshirani. Linear smoothers and additive models. The Annals of Statistics, 17:453-510. Citado na pág.

Cao et al.(2010) C.Z. Cao, J.G. Lin e L.X. Zhu. Heteroscedasticity and/or autocorrelation diagnostics in nonlinear models with ar (1) and symmetrical errors. Statistical Papers, 51(4):813-836. Citado na pág. 2, 34, 35, 103

Chen e You(2005) G. Chen e J. You. An asymptotic theory for semiparametric generalized least squares estimation in partially linear regression models. Statistical Papers, 46(2):173-193. Citado na pág. 3

Cook(1977) R.D. Cook. Detection of influential observation in linear regression. Technometrics, 19:15-18. Citado na pág. 
Cook(1986) R.D. Cook. Assessment of local influence. Journal of the Royal Statistical Society. Series B (Methodological), 48:133-169. Citado na pág. 3, 49, 52,53

Cook e Weisberg(1982) R.D. Cook e S. Weisberg. Residuals and Influence in Regression. Chapman and Hall. Citado na pág.

Cook e Weisberg(1983) R.D. Cook e S. Weisberg. Diagnostics for heteroscedasticity in regression. Biometrika, 70(1):1-10. Citado na pág. 2

Cox e Snell(1968) D.R. Cox e E.J. Snell. A general definition of residuals. Journal of the Royal Statistical Society. Series B (Methodological), 30:248275. Citado na pág.

Cysneiros(2004) F. J. A. Cysneiros. Métodos Restritos e Validação de Modelos Simétricos de Regressão. Tese de Doutorado, Departamento de Estatística, Universidade de São Paulo, Brasil. Citado na pág. 20, 23, 25

Cysneiros e Paula(2005) F.J.A. Cysneiros e G.A. Paula. Restricted methods in symmetrical linear regression models. Computational Statistics 6) Data Analysis, 49(3):689-708. Citado na pág. 1

Cysneiros et al.(2005) F.J.A. Cysneiros, G.A. Paula e M. Galea. Modelos Simétricos Aplicados. Águas de São Pedro. Citado na pág. 2

Cysneiros et al.(2007) F.J.A. Cysneiros, G.A. Paula e M. Galea. Heteroscedastic symmetrical linear models. Statistics $\&$ Probability Letters, 77(11): 1084-1090. Citado na pág. 1

Dabo-Niang e Guillas(2010) S. Dabo-Niang e S. Guillas. Functional semiparametric partially linear model with autoregressive errors. Journal of Multivariate Analysis, 101(2):307-315. Citado na pág. 3

Eubank(1984) RL Eubank. The hat matrix for smoothing splines. Statistics 86 Probability Letters, 2(1):9-14. Citado na pág.

Eubank(1985) RL Eubank. Diagnostics for smoothing splines. Journal of the Royal Statistical Society. Series B (Methodological), 47:332-341. Citado na pág.

Eubank(1988) R.L. Eubank. Spline Smoothing and Nonparametric Regression. Marcel Dekker. Citado na pág. 
Eubank e Gunst(1986) RL Eubank e RF Gunst. Diagnostics for penalized least-squares estimators. Statistics $\&$ Probability Letters, 4(5):265-272. Citado na pág.

Eubank e Thomas(1993) RL Eubank e W. Thomas. Detecting heteroscedasticity in nonparametric regression. Journal of the Royal Statistical Society. Series B (Methodological), 55:145-155. Citado na pág.

Fang e Anderson(1990) H.T. Fang e T.W. Anderson. Statistical Inference in Elliptically Contoured and Related Distributions. Alerton Press. Citado na pág. 20

Fang et al.(1990) H.T Fang, S. Kotz e W. Ng. Symmetric Multivariate and Related Distribution. Chapman and Hall. Citado na pág. 1, 20

Galea et al.(1997) M. Galea, G.A. Paula e H. Bolfarine. Local influence in elliptical linear regression models. Journal of the Royal Statistical Society: Series D (The Statistician), 46(1):71-79. Citado na pág.

Galea et al.(2003) M. Galea, G.A. Paula e M. Uribe-Opazo. On influence diagnostic in univariate elliptical linear regression models. Statistical Papers, 44(1):23-45. Citado na pág. 1

Galea et al.(2005) M. Galea, G.A. Paula e F.J.A. Cysneiros. On diagnostics in symmetrical nonlinear models. Statistics \& Probability Letters, 73(4): 459-467. Citado na pág. 1

Green(1987) P.J. Green. Penalized likelihood for general semi-parametric regression models. International Statistical Review/Revue Internationale de Statistique, 55:245-259. Citado na pág.

Green e Silverman(1994) P.J. Green e B.W. Silverman. Nonparametric Regression and Generalized Linear Models. Boca Raton. Citado na pág. 2, 10, $16,17,18,45,98$

Hamilton(1994) J.D. Hamilton. Time Series Analysis. Cambridge Univ Press. Citado na pág. 98

Hastie e Tibshirani(1990) T. Hastie e R. Tibshirani. Generalized Additive Models. Chapman and Hall. Citado na pág. 
Heckman(1986) N.E. Heckman. Spline smoothing in a partly linear model. Journal of the Royal Statistical Society. Series B (Methodological), 48:244248. Citado na pág.

Heckman(1988) N.E. Heckman. Minimax estimates in a semiparametric model. Journal of the American Statistical Association, 83:1090-1096. Citado na pág.

Huber(1973) P.J. Huber. Robust regression: asymptotics, conjectures and monte carlo. The Annals of Statistics, 1(5):799-821. Citado na pág. 19

Hurvich et al.(1998) C.M. Hurvich, J.S. Simonoff e C.L. Tsai. Smoothing parameter selection in nonparametric regression using an improved akaike information criterion. Journal of the Royal Statistical Society: Series B (Statistical Methodology), 60(2):271-293. Citado na pág.

Ibacache-Pulgar e Paula(2011) G. Ibacache-Pulgar e G.A. Paula. Local influence for student-t partially linear models. Computational Statistics $\&$ Data Analysis, 55(3):1462-1478. Citado na pág. 2, 3

Ibacache-Pulgar(2009) G.M. Ibacache-Pulgar. Modelos Mistos Aditivos Semiparamétricos de Contornos Elípticos. Tese de Doutorado, Instituto de Matemática e Estatística, Departamento de Estatística, Universidade de São Paulo, Brasil. Citado na pág. 42, 44, 45

Ibacache-Pulgar et al.(2012) G.M. Ibacache-Pulgar, G. Paula e F. Cysneiros. Semiparametric additive models under symmetric distributions. TEST, páginas 1-19. Citado na pág. 19, 25

Kim et al.(2002) C. Kim, B.U. Park e W. Kim. Influence diagnostics in semiparametric regression models. Statistics \& Probability Letters, 60(1): 49-58. Citado na pág. 2

Lange et al.(1989) K.L. Lange, R.J.A. Little e J.M.G. Taylor. Robust statistical modeling using the t distribution. Journal of the American Statistical Association, 84:881-896. Citado na pág. 1

Lesaffre e Verbeke(1998) E. Lesaffre e G. Verbeke. Local influence in linear mixed models. Biometrics, 38:570-582. Citado na pág. 54

Lin e Wei(2004) J.G. Lin e B.C. Wei. Testing for heteroscedasticity and/or autocorrelation in nonlinear regression models with correlated errors. Commum Stat Theory Methods, 33:251-275. Citado na pág. 2 
Lin e Wei(2003) J.G. Lin e B.C. Wei. Testing for heteroscedasticity in nonlinear regression models. Communications in Statistics-Theory and Methods, 32(1):171-192. Citado na pág. 2

Liu(2000) S. Liu. On local influence for elliptical linear models. Statistical Papers, 41(2):211-224. Citado na pág.

Liu(2002) S. Liu. Local influence in multivariate elliptical linear regression models. Linear Algebra and Its Applications, 354(1):159-174. Citado na pág.

Lucas(1997) A. Lucas. Robustness of the student t based m-estimator. Communications in Statistics-Theory and Methods, 26:1165-1182. Citado na pág. 79

McCullagh e Nelder(1989) P. McCullagh e J.A. Nelder. Generalized Linear Models. Chapman \& Hall/CRC. Citado na pág.

Medeiros(2006) M. J. Medeiros. Métodos de diagnóstico em modelos autoregressivos simétricos. Dissertação de Mestrado, Instituto de Matemática e Estatística, Departamento de Estatística, Universidade de São Paulo, Brasil. Citado na pág. 102

Montgomery et al.(2007) D.C. Montgomery, E.A. Peck e G.G. Vining. Introduction to Linear Regression Analysis, volume 49. John Wiley \& Sons. Citado na pág. 1, 2

Morettin(2011) P. A. Morettin. Econometria Financeira - Um Curso em Séries Temporais Financeiras. Editora Edgard Blucher. Citado na pág.

Morettin e Toloi(2006) P. A. Morettin e C. M. C. Toloi. Análise de Séries Temporais. Editora Edgard Blucher. Citado na pág. 63

Neter et al.(1996) J. Neter, C.J. Nachtsheim e M.H. Kutner. Applied Linear Regression Models. Irwin, Chicago. Citado na pág. 26, 31

Ortega et al.(2003) E.M.M. Ortega, H. Bolfarine e G.A. Paula. Influence diagnostics in generalized log-gamma regression models. Computational Statistics $\&$ Data Analysis, 42(1):165-186. Citado na pág.

Osorio et al.(2007) F. Osorio, G.A. Paula e M. Galea. Assessment of local influence in elliptical linear models with longitudinal structure. Computational Statistics 85 Data Analysis, 51(9):4354-4368. Citado na pág. 
Otsu(2007) T. Otsu. Penalized empirical likelihood estimation of semiparametric models. Journal of Multivariate Analysis, 98(10):1923-1954. Citado na pág.

Paula(2010) G.A. Paula. Modelos de Regressão com Apoio Computacional. Instituto de Matemática e Estatística da Universidade de São Paulo. Citado na pág. $3,7,50,52$

Paula(1993) G.A. Paula. Assessing local influence in restricted regression models. Computational Statistics \& Data Analysis, 16(1):63-79. Citado na pág.

Paula e Cysneiros(2009) G.A. Paula e F.J.A. Cysneiros. Systematic risk estimation in symmetric models. Applied Economics Letters, 16(2):217-221. Citado na pág. 2

Paula et al.(2003) G.A. Paula, F.J.A. Cysneiros e M. Galea. Local influence and leverage in elliptical nonlinear regression models. Em Proceedings of the 18th International Workshop on Statistical Modelling. Katholieke Universiteit Leuven, Leuven, páginas 361-365. Citado na pág.

Paula et al.(2009) G.A. Paula, M. Medeiros e F.E. Vilca-Labra. Influence diagnostics for linear models with first-order autoregressive elliptical errors. Statistics \& Probability Letters, 79(3):339-346. Citado na pág. 3

Poon e Poon(1999) W.Y. Poon e Y.S. Poon. Conformal normal curvature and assessment of local influence. Journal of the Royal Statistical Society: Series B (Statistical Methodology), 61(1):51-61. Citado na pág. 54

Rigby e Stasinopoulos(2005) RA Rigby e DM Stasinopoulos. Generalized additive models for location, scale and shape. Journal of the Royal Statistical Society: Series C (Applied Statistics), 54(3):507-554. Citado na pág. 2, 42

Ruppert(2004) D. Ruppert. Statistics and Finance: an Introduction. Springer Verlag. Citado na pág. 10

Ruppert et al.(2003) D. Ruppert, M.P. Wand e R.J. Carroll. Semiparametric Regression. Cambridge Univ Pr. Citado na pág. 10, 12, 16, 17

Russo(2006) C. M. Russo. Análise de um modelo de regressão com erros nas variáveis multivariado com intercepto nulo. Dissertação de Mestrado, Universidade de São Paulo - São Carlos. Citado na pág. 
Schwarz(1978) G. Schwarz. Estimating the dimension of a model. The Annals of Statistics, 6(2):461-464. Citado na pág.

Silverman(1982) B.W. Silverman. On the estimation of a probability density function by the maximum penalized likelihood method. The Annals of Statistics, 10:795-810. Citado na pág.

Silverman(1984) B.W. Silverman. A fast and efficient cross-validation method for smoothing parameter choice in spline regression. Journal of the American Statistical Association, 79:584-589. Citado na pág.

Silverman(1985) B.W. Silverman. Some aspects of the spline smoothing approach to non-parametric regression curve fitting. Journal of the Royal Statistical Society. Series B (Methodological), 47:1-52. Citado na pág.

Simonoff e Tsai(1999) J.S. Simonoff e C.L. Tsai. Semiparametric and additive model selection using an improved akaike information criterion. Journal of Computational and Graphical Statistics, 8:22-40. Citado na pág.

Taylor e Verbyla(2004) J. Taylor e A. Verbyla. Joint modeling of location and scale parameters of t distribution. Statistical Modelling, 4:91-112. Citado na pág. 2

Thomas(1991) W. Thomas. Influence diagnostics for the cross-validated smoothing parameter in spline smoothing. Journal of the American Statistical Association, 86:693-698. Citado na pág.

Tsai(1986) C.L. Tsai. Score test for the first-order autoregressive model with heteroscedasticity. Biometrika, 73(2):455-460. Citado na pág. 2

Tsai e Wu(1992) C.L. Tsai e X. Wu. Assessing local influence in linear regression models with first-order autoregressive or heteroscedastic error structure. Statistics $\&$ Probability Letters, 14(3):247-252. Citado na pág.

Verbyla(1993) A.P. Verbyla. Modelling variance heterogeneity: residual maximum likelihood and diagnostics. Journal of the Royal Statistical Society. Series B (Methodological), 55:493-508. Citado na pág. 2

Wei et al.(1998) B.C. Wei, Y.Q. Hu e W.K. Fung. Generalized leverage and its applications. Scandinavian Journal of Statistics, 25(1):25-37. Citado na pág. 56 
Wei(2004) W.H. Wei. Derivatives diagnostics and robustness for smoothing splines. Computational Statistics \&6 Data Analysis, 46(2):335-356. Citado na pág.

Zhu e Zhang(2004) H. Zhu e H. Zhang. A diagnostic procedure based on local influence. Biometrika, 91(3):579-589. Citado na pág. 54

Zhu et al.(2007) H. Zhu, J.G. Ibrahim, S. Lee e H. Zhang. Perturbation selection and influence measures in local influence analysis. The Annals of Statistics, 35:2565-2588. Citado na pág.

Zhu et al.(2003) Z.Y. Zhu, X. He e W.K. Fung. Local influence analysis for penalized gaussian likelihood estimators in partially linear models. Scandinavian Journal of Statistics, 30(4):767-780. Citado na pág. 